ANL/ER/TR-01/003

\title{
Deposition Parameterizations for the Industrial Source Complex (ISC3) Model
}

by M. L. Wesely, P. V. Doskey, and J. D. Shannon

Environmental Research Division, Argonne National Laboratory

9700 South Cass Avenue

Argonne, Illinois 60439

June 2002

Work sponsored by the U.S. Department of Energy, Office of Science, Office of Biological and Environmental Research, Environmental Sciences Division and partially by the U.S. Environmental Protection Agency, Office of Air Quality Planning and Standards. Argonne National Laboratory is operated by The University of Chicago for the U.S. Department of Energy under contract W-31-109-Eng38. 


\section{Summary}

Improved algorithms have been developed to simulate the dry and wet deposition of hazardous air pollutants (HAPs) with the Industrial Source Complex version 3 (ISC3) model system. The dry deposition velocities (concentrations divided by downward flux at a specified height) of the gaseous HAPs are modeled with algorithms adapted from existing dry deposition modules. The dry deposition velocities are described in a conventional resistance scheme, for which micrometeorological formulas are applied to describe the aerodynamic resistances above the surface. Pathways to uptake at the ground and in vegetative canopies are depicted with several resistances that are affected by variations in air temperature, humidity, solar irradiance, and soil moisture. The role of soil moisture variations in affecting the uptake of gases through vegetative plant leaf stomata is assessed with the relative available soil moisture, which is estimated with a rudimentary budget of soil moisture content. Some of the procedures and equations are simplified to be commensurate with the type and extent of information on atmospheric and surface conditions available to the ISC3 model system user. For example, standardized land use types and seasonal categories provide sets of resistances to uptake by various components of the surface. To describe the dry deposition of the large number of gaseous organic HAPS, a new technique based on laboratory study results and theoretical considerations has been developed providing a means of evaluating the role of lipid solubility in uptake by the waxy outer cuticle of vegetative plant leaves.

The dry deposition velocities of particulate HAPs are simulated with a resistance scheme in which the depositional properties of particles are described with formulations previously developed for ISC models except, for a modification to one equation to describe deposition velocity more accurately in strongly unstable conditions. For situations in which coarse particles are not present in significant amounts, a simpler scheme is recommended in which deposition velocity is described for only two size modes: a fine mode with particles less than about $2.5 \mu \mathrm{m}$ in diameter and a coarse mode including larger particles but excluding very coarse particles larger than about $10 \mu \mathrm{m}$ in diameter. For the fine mode, the deposition velocity is calculated with a parameterization based on observations of sulfate dry deposition. For the coarse mode, a representative settling velocity is assumed. Then the total deposition velocity is estimated as the sum of the two deposition velocities, weighted according to the amount of mass expected in the two modes. Representative values of the relative masses in the two size modes are derived for each particulate HAP from observations reported in the scientific literature for urban or industrial areas. These representative values are not specific to any of the wide variety of sources from which HAPs are emitted.

Wet deposition is described in terms of washout ratios based in part on theory but more strongly on observations reported in the scientific literature. The washout ratios do not account for in-cloud processes, because the short-range dispersion of plumes considered with the ISC3 modeling system is assumed to be below cloud level. The flux is expressed in terms of the concentration of the HAP, the precipitation rate, and the washout ratio. For gases, the washout ratio is a unitless version of the Henry's Law constant, which is most appropriate for chemical equilibrium conditions in a well-mixed planetary boundary layer. For particles, the washout ratio is assumed to be proportional to the mass median diameter of the particulate substance, which 
represents an approximation well within uncertainties in modeling and observational results. The mass median diameters are estimated on the basis of the split in mass between the fine and the coarse modes.

Application of the deposition algorithms for HAPs requires considerable information on their properties. A means to evaluate the partitioning between the gaseous and particulate phases of organic HAPs requires description of a process that depends heavily on properties that can vary greatly from substance to substance. The amount of mass in the two phases is described in terms of the concentration of total suspended particulate matter (TSP) in the atmosphere and the octanol-air partition coefficient for the substance of interest. Because local measurements of TSP might not be available to the ISC3 modeling system user, a default value for TSP is suggested, equal to the national air quality standard for particulate mass less than $10 \mu \mathrm{m}$ in diameter. In addition, estimates are needed of the values of physicochemical properties of the gaseous HAPs, including Henry's Law constants, molecular diffusivities in air and water, the octanol-air partition coefficients, and the octanol-water partition coefficients. Many of the values for the organic compounds were derived from published compilations and from some detailed calculations. Some gaps remain in this information, and a special effort in computational chemistry might be needed to generate selected values of the physiochemical properties of some of the organic HAPs. 


\section{Contents}

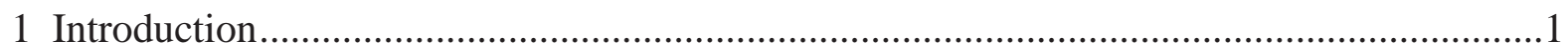

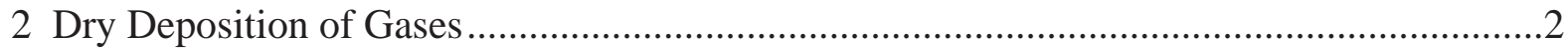

2.1 Gas-Phase Resistances ..........................................................................

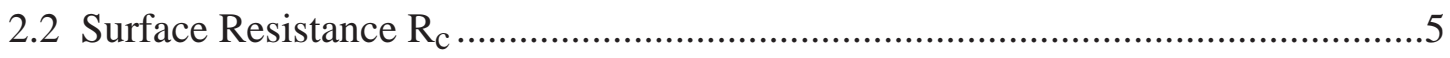

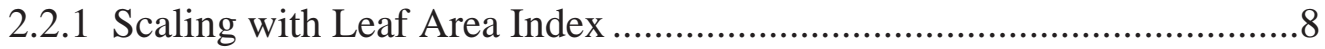

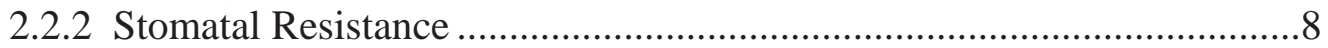

2.2.3 Mesophyll, Cuticular, and Ground Resistances................................11

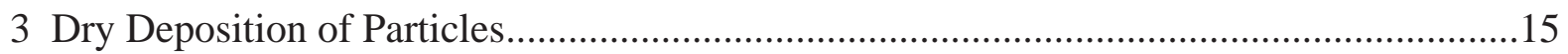

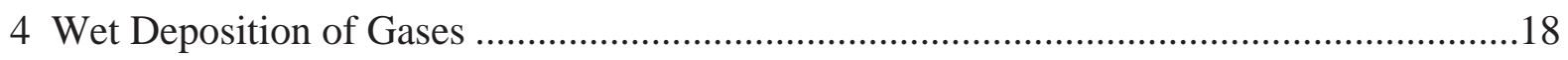

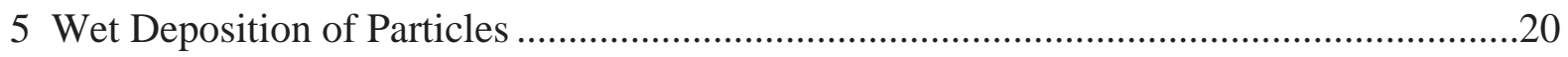

6 Physicochemical Properties and Related Topics ........................................................22

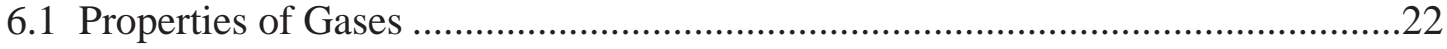

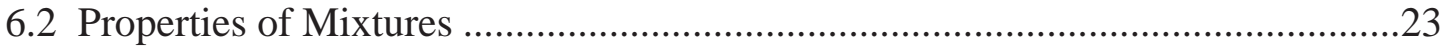

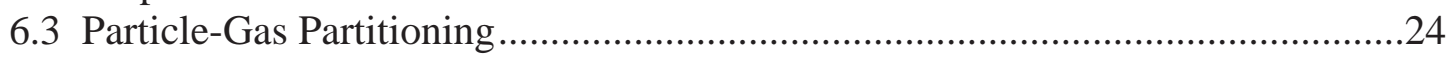

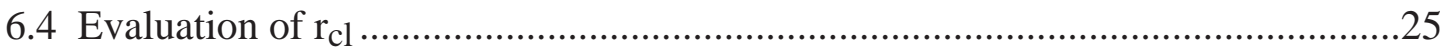

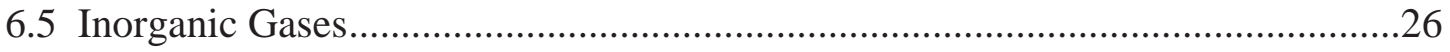

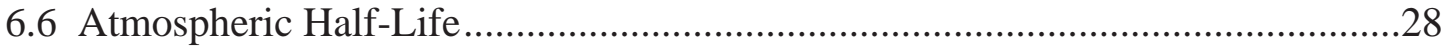

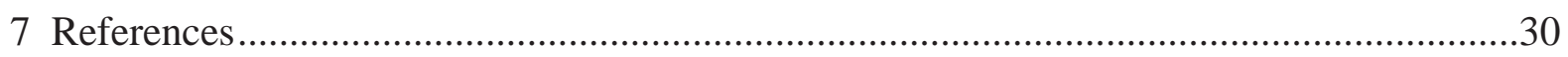

APPENDIX A: Summary of Equations Necessary in Model ........................................... A-1

APPENDIX B: Particle Size Information for Selected Hazardous Air Pollutants ...............B-1

APPENDIX C: Basic Physicochemical Properties of Gaseous Hazardous Air Pollutants

APPENDIX D: Additional Physicochemical Properties of Gaseous Hazardous Air Pollutants ................................................................................... D-1

APPENDIX E: Physicochemical Properties of Mixtures of Hazardous Air Pollutants .......E-1

APPENDIX F: Estimates of Gas-Phase Lifetimes for Selected Substances ......................F-1 


\section{Figures}

1 Scheme of major components of resistances used for gaseous dry deposition

\section{Tables}

$1 \quad$ Land use types and seasonal categories ........................................................

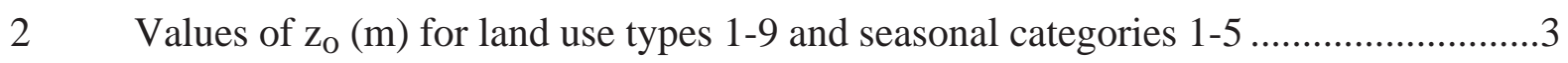

$3 \quad$ Values of surface resistances for model inputs...............................................

$4 \quad$ Values of the factor $\mathrm{S}$ to scale leaf resistance $\mathrm{r}_{\mathrm{cl}}$ to uptake associated with lipid solubility, to estimate leaf resistance $\mathrm{R}_{\mathrm{cl}}$ for entire canopies ..................................12

$5 \quad$ Atomic diffusion volumes for the calculation of molecular volumes $\sum_{\mathrm{v}} \ldots \ldots \ldots \ldots \ldots \ldots . . .23$

6 Volume increments for the calculation of the Le Bas molar volumes V ...................24

$7 \quad$ Suggested values of key physicochemical properties of inorganic gases..................27 


\section{Introduction}

This report describes updates and improvements recommended for algorithms used to describe dry and wet deposition in the Industrial Source Complex version 3 (ISC3) model system. Estimates of the amounts of gaseous and particulate material deposited by wet and dry processes on outdoor surfaces are necessary to assess the impacts of toxic air pollutants. This report focuses on the parameterization of deposition for the wide variety of types of substances listed as hazardous air pollutants (HAPs). Appendix A summarizes the equations generated for use in the ISC3 model system, and Appendices B-F provide tabulations of the values of the physical and chemical properties needed for application of the equations. Implementation of the new algorithms would leave all of the previous capabilities of the ISC3 model system intact, for example, including deposition and plume depletion of gases and particles, as well as deposition of both fine and large particles and wet deposition during rain and snow.

The ISC3 model system relies on a steady-state Gaussian plume model to simulate shortrange dispersion (Environmental Protection Agency, 1995a,b). Most of the algorithms described in this document could also be applied to other types of air quality models, although the algorithms might need to be altered for models based on computational schemes that differ significantly from those in the ISC3 model system or that use different observational input data. The deposition algorithms are designed to use micrometeorological parameters evaluated by the ISC3 meteorological preprocessor, which is driven by standard surface meteorological observations of the kind taken routinely by the National Weather Service.

The development of deposition algorithms for HAPs and ISC3 has some significant difficulties and practical considerations. A difficulty is that the physicochemical properties of many of the organic HAPs are not well known. An extensive literature search was conducted for this report, and some detailed computations were made, but gaps remain in data needed to apply algorithms. A special computational chemistry effort might be needed to generate values of the physiochemical properties of some of the organic HAPs. A practical consideration is that the information available to ISC3 model system users on local surface conditions is usually limited. Standardized options are necessary to characterize some of the surface conditions that affect deposition in specific areas to which ISC3 is applied. As a result, relatively simple algorithms for dry deposition, which is usually highly sensitive to surface conditions, are likely to produce deposition estimates that are as reliable as results from more sophisticated algorithms. Another limitation is that rapid chemical reactions and interactions between gases and particles can be significant within very short distances from certain types of sources, so that characteristics of the HAPs downwind (e.g., particle size distribution can be difficult to determine. Some special provisions are suggested in this report to deal with these practical considerations and limitations.

In this report, the ISC3 model system is hereafter referred to as "ISC." Frequent reference is also made to the deposition algorithms in the Models-3 Community Multiscale Air Quality (Byun et al., 1999), which is identified as "Models-3," and to the dry deposition module developed for the Regional Acid Deposition Model (Wesely, 1989), which is identified as "RADM." 


\section{Dry Deposition of Gases}

The deposition velocity $\mathrm{V}_{\mathrm{dg}}$ is the modeling parameter used to describe gaseous dry deposition. This velocity is designed to be multiplied by the modeled concentration at a specified height near the surface, to estimate the mass flux density (mass per unit time per unit area) to the surface. Many of the equations to evaluate $\mathrm{V}_{\mathrm{dg}}$ for inorganic gases can be found in existing dry deposition models (e.g., Wesely 1989; Erisman 1994; Byun et al. 1999). However, work on the deposition velocities for most of the gaseous HAPs is limited. Because field experiments have not been conducted to evaluate the deposition of the gaseous HAPs quantitatively, development of a description of the deposition velocities must rely considerably on findings from laboratory studies and theoretical considerations, and the accuracy of the resulting equations cannot be fully determined. New parameterizations are developed here, for example, to describe the role of lipid solubility in affecting the bulk vegetative canopy uptake of organic substances by leaf cuticles, but the resulting formulations have not been tested with field observations.

The formulations for deposition velocities incorporate several variables whose values are determined by land use type and seasonal category (Tables 1 and 2), the selection of which requires care and judgement. In general, if a particular receptor area is being considered, the land use type should conform to the surface conditions that actually exist in that area. For example, for agricultural areas during the summer, seasonal category 3 would be preferred over category 1 if the surface in the area is bare soil. The seasonal categories correspond roughly to calendar seasons for the northern, noncoastal portions of the United States. At some southern latitudes, categories 3 and 4 might never be used; category 1 might be the only suitable choice for southern Florida.

\subsection{Gas-Phase Resistances}

The deposition velocities of the gaseous HAPs of interest are estimated with a conventional resistance scheme:

$$
\mathrm{V}_{\mathrm{dg}}=\left(\mathrm{R}_{\mathrm{a}}+\mathrm{R}_{\mathrm{b}}+\mathrm{R}_{\mathrm{c}}\right)^{-1}
$$

Here $\mathrm{R}_{\mathrm{a}}$ represents the aerodynamic resistance to vertical transport below a specified height above the surface, $R_{b}$ is the surface resistance of the quasilaminar sublayer of air in contact with surface elements, and $\mathrm{R}_{\mathrm{c}}$ is the resistance of the surface itself to uptake. All three of the resistances represent bulk properties of the air and surface and are expressed in units of seconds per meter. The resistances $\mathrm{R}_{\mathrm{a}}$ and $\mathrm{R}_{\mathrm{b}}$ are controlled entirely by the physical processes of turbulent mixing and diffusion and are described with conventional micrometeorological equations. 
Table 1. Land use types and seasonal categories.

Land use

No. Type

1. Urban land

2. Agricultural land

3. Rangeland

4. Forest

5. Suburban areas, grassy

6. Suburban areas, forested

7. Bodies of water

8. Barren land, mostly desert

9. Nonforested wetlands
Seasonal Category

No. Description
1. Midsummer with lush vegetation

2. Autumn with unharvested cropland

3. Late autumn after frost, no snow

4. Winter with snow on ground

5. Transitional spring with partial green coverage or short annuals

Table 2. Values of $z_{0}(m)$ for land use types 1-9 and seasonal categories 1-5.

\begin{tabular}{|c|c|c|c|c|c|c|c|c|c|}
\hline \multirow{2}{*}{$\begin{array}{l}\text { Seasonal } \\
\text { category }\end{array}$} & \multicolumn{9}{|c|}{ Land use type } \\
\hline & 1 & 2 & 3 & 4 & 5 & 6 & 7 & 8 & 9 \\
\hline 1. & 1.0 & 0.25 & 0.05 & 1.0 & 0.6 & 1.0 & 0.0001 & 0.002 & 0.15 \\
\hline 2. & 1.0 & 0.10 & 0.05 & 1.0 & 0.6 & 1.0 & 0.0001 & 0.002 & 0.10 \\
\hline 3. & 1.0 & 0.005 & 0.05 & 1.0 & 0.6 & 1.0 & 0.0001 & 0.002 & 0.10 \\
\hline 4. & 1.0 & 0.001 & 0.001 & 1.0 & 0.6 & 1.0 & 0.0001 & 0.002 & 0.001 \\
\hline 5. & 1.0 & 0.03 & 0.02 & 1.0 & 0.6 & 1.0 & 0.0001 & 0.002 & 0.10 \\
\hline
\end{tabular}


The term $R_{a}$ is evaluated for atmospherically stable and neutral conditions ( $L \geq 0$ ) as follows:

$$
\mathrm{R}_{\mathrm{a}}=\left(\mathrm{ku}_{*}\right)^{-1}\left[\ln \left(\mathrm{z}_{\mathrm{r}} / \mathrm{z}_{\mathrm{O}}\right)+5 \mathrm{z}_{\mathrm{r}} / \mathrm{L}\right]
$$

For unstable conditions ( $\mathrm{L}<0$ ), the following formula is applied:

$$
\mathrm{R}_{\mathrm{a}}=\left(\mathrm{ku}_{*}\right)^{-1}\left\{\ln \left(\mathrm{z}_{\mathrm{r}} / \mathrm{z}_{\mathrm{o}}\right)-2 \ln \left[\frac{1}{2}\left(1+\sqrt{1-16 \mathrm{z}_{\mathrm{r}} / \mathrm{L}}\right)\right]+2 \ln \left[\frac{1}{2}\left(1+\sqrt{1-16 \mathrm{z}_{\mathrm{o}} / \mathrm{L}}\right)\right]\right\},
$$

where

$$
\begin{array}{ll}
\mathrm{k} & =\text { the von Karman constant }(0.4), \\
\mathrm{u}_{*} & =\text { friction velocity }\left(\mathrm{m} \mathrm{s}^{-1}\right), \\
\mathrm{Z}_{\mathrm{r}} & =\text { reference height }(\mathrm{m}), \\
\mathrm{Z}_{\mathrm{O}} & =\text { surface roughness length scale }(\mathrm{m}), \\
\mathrm{L} & =\text { Monin-Obukhov length scale }(\mathrm{m}) .
\end{array}
$$

Equations (2a) and (2b) contain widely used forms for adjustments for non-neutral atmospheric stability (e.g., Panofsky and Dutton, 1984), although the third term with the form $16 \mathrm{z}_{\mathrm{O}} / \mathrm{L}$ in the large parentheses on the right-hand side of equation (2b) is often omitted. Equation ( $2 b)$ is equivalent to a form (e.g., Wesely, 1979; Byun, 1990; Byun and Dennis, 1995) that has been applied previously in ISC and in computationally intensive models:

$$
\mathrm{R}_{\mathrm{a}}=\left(\mathrm{ku}_{*}\right)^{-1}\left\lfloor\ln \frac{\left(\sqrt{1-16 \mathrm{z}_{\mathrm{r}} / \mathrm{L}}-1\right)\left(\sqrt{1-16 \mathrm{z}_{\mathrm{o}} / \mathrm{L}}+1\right)}{\left(\sqrt{1-16 \mathrm{z}_{\mathrm{r}} / \mathrm{L}}+1\right)\left(\sqrt{1-16 \mathrm{z}_{\mathrm{o}} / \mathrm{L}}-1\right)}\right\rfloor
$$

Estimates of $\mathrm{L}, \mathrm{u}_{*}, \mathrm{z}_{\mathrm{O}}$, and $\mathrm{z}_{\mathrm{r}}$ are provided by the meteorological preprocessor of ISC. Because vegetative conditions can vary greatly from site to site, however, the values of $z_{r}$ and $z_{0}$ should be examined to ensure that they are representative of local conditions. The term $z_{r}$ represents the height above the zero plane displacement height $d$, which can be estimated as $2 / 3$ of the average height $\mathrm{h}$ of the vegetation present, rather than the height $\mathrm{z}$ above the soil surface. That is, the reference height can be found as $\mathrm{z}_{\mathrm{r}}=\mathrm{z}-0.67 \mathrm{~h}$. For suburban and industrial areas with many buildings, a displacement height of about $\mathrm{d}=4 \mathrm{~m}$ is typical (Grimmond et al., 1998).

Values of $\mathrm{z}_{\mathrm{O}}$ can be selected according to land use type (Table 1); Table 2 shows the corresponding set of default values for $\mathrm{z}_{\mathrm{O}}$. As rules of thumb, $\mathrm{z}_{\mathrm{O}}$ should be approximately equal to $0.08 \mathrm{~h}$ for grassy vegetation including maize and $0.05 \mathrm{~h}$ for soybeans, similar dicotyledons, and forested areas (e.g., Brutsaert, 1982). If the value of $\mathrm{z}_{\mathrm{O}}$ assumed in the ISC meteorological preprocessor is substantially different, say by a factor of 2 or more, from $z_{0}$ listed in Table 2 or inferred on the basis of the height of vegetation height, the value of $\mathrm{u}_{*}$ from the meteorological preprocessor will probably not be representative of local conditions. In this situation, an improved estimate of $\mathrm{u}_{*}$ should be made and used to calculate $\mathrm{R}_{\mathrm{a}}$ via equation (2) and $\mathrm{R}_{\mathrm{b}}$ (equation shown below). The new estimate of $\mathrm{u}_{*}$ can be made with the following relationship, derived from micrometeorological equations and the rough approximation that the product of $\mathrm{u}_{*}$ and mean wind velocity at a height of several meters is constant over diverse types of surfaces in a region at a given time: 


$$
\mathrm{u}_{*}=\left[\mathrm{ku}_{\mathrm{m}} \mathrm{u}_{* \mathrm{~m}} / \ln \left(\mathrm{z}_{\mathrm{rm}} / \mathrm{z}_{\mathrm{o}}\right)\right]^{1 / 2}
$$

where $\quad \mathrm{u}_{*} \quad=$ new friction velocity $\left(\mathrm{m} \mathrm{s}^{-1}\right)$,

$\mathrm{k}=$ the von Karman constant (0.4),

$\mathrm{u}_{\mathrm{m}} \quad=$ wind speed from preprocessor $\left(\mathrm{m} \mathrm{s}^{-1}\right)$,

$\mathrm{u}_{* \mathrm{~m}}=$ friction velocity from preprocessor $\left(\mathrm{m} \mathrm{s}^{-1}\right)$,

$\mathrm{Z}_{\mathrm{rm}} \quad=$ reference height used in preprocessor (usually $10 \mathrm{~m}$ ),

$\mathrm{z}_{\mathrm{O}} \quad=\quad$ local surface roughness length scale $(\mathrm{m})$.

Estimates of $R_{b}$ are found from the following formula:

$$
\mathrm{R}_{\mathrm{b}}=2.2\left(\mathrm{v} / \mathrm{D}_{\mathrm{a}}\right)^{2 / 3}\left(\mathrm{ku}_{*}\right)^{-1}
$$

where $v$ is kinematic viscosity of air (approximately $0.1505 \times 10^{-4} \mathrm{~m}^{2} \mathrm{~s}^{-1}$ ), and $\mathrm{D}_{\mathrm{a}}$ is the diffusivity of the gas of interest in air (evaluated as described in Section 6.1). This formula for $\mathrm{R}_{\mathrm{b}}$ assumes that the surface is "fibrous" and fairly even, as opposed to having relatively solid surface elements, such as a highly ridged plowed field, vineyards, a field with isolated trees, and urban areas.

\subsection{Surface Resistance $R_{C}$}

Resistance $\mathrm{R}_{\mathrm{c}}$ involves a number of physical, chemical, and biological processes and is usually the most difficult of the resistances in equation (1) to evaluate accurately. A simplified and slightly modified version of the schemes used in various resistance models is appropriate (Wesely 1989; Erisman 1994; Byun et al. 1999). Figure 1 shows the recommended scheme. The arrangement of resistances in Figure 1 leads to the formulation

$$
\mathrm{R}_{\mathrm{c}}=\left[\mathrm{LAI}_{\mathrm{r}}\left(\mathrm{R}_{\mathrm{s}}+\mathrm{R}_{\mathrm{m}}\right)^{-1}+\mathrm{LAI}_{\mathrm{r}} \mathrm{R}_{\mathrm{cut}}{ }^{-1}+\left(\mathrm{R}_{\mathrm{ac}}+\mathrm{R}_{\mathrm{g}}\right)^{-1}\right]^{-1}
$$

where

$$
\begin{aligned}
& \mathrm{LAI}_{\mathrm{r}}=\text { relative leaf area index (unitless), } \\
& \mathrm{R}_{\mathrm{S}}=\text { canopy stomatal resistance }\left(\mathrm{s} \mathrm{m}^{-1}\right), \\
& \mathrm{R}_{\mathrm{m}}=\text { canopy mesophyll resistance }\left(\mathrm{s} \mathrm{m}^{-1}\right), \\
& \mathrm{R}_{\mathrm{cut}}=\text { canopy cuticular resistance }\left(\mathrm{s} \mathrm{m}^{-1}\right), \\
& \mathrm{R}_{\mathrm{ac}}=\text { gas-phase resistance in the vegetative canopy }\left(\mathrm{s} \mathrm{m}^{-1}\right), \\
& \mathrm{R}_{\mathrm{g}} \quad=\text { resistance to uptake at the ground }\left(\mathrm{s} \mathrm{m}^{-1}\right) .
\end{aligned}
$$

The relative leaf area index $\mathrm{LAI}_{\mathrm{r}}$ is described in Section 2.2.1. All of the resistances indicated by a upper-case "R" indicate a bulk property of the vegetative canopy and the surface underneath. That is, these bulk resistances represent an integral of surface resistances, even though the uptake processes actually occur at individual surface elements. For example, $\mathrm{R}_{\mathrm{S}}$ accounts for resistance to molecular diffusion through the leaf stomatal apertures, $R_{m}$ indicates resistance to uptake by mesophyll cells in the substomatal cavities of the leaves, $\mathrm{R}_{\text {cut }}$ deals with the uptake at the waxy outer cuticle that covers leaves, and $R_{g}$ is the resistance to uptake at the bare soil and plant litter. 


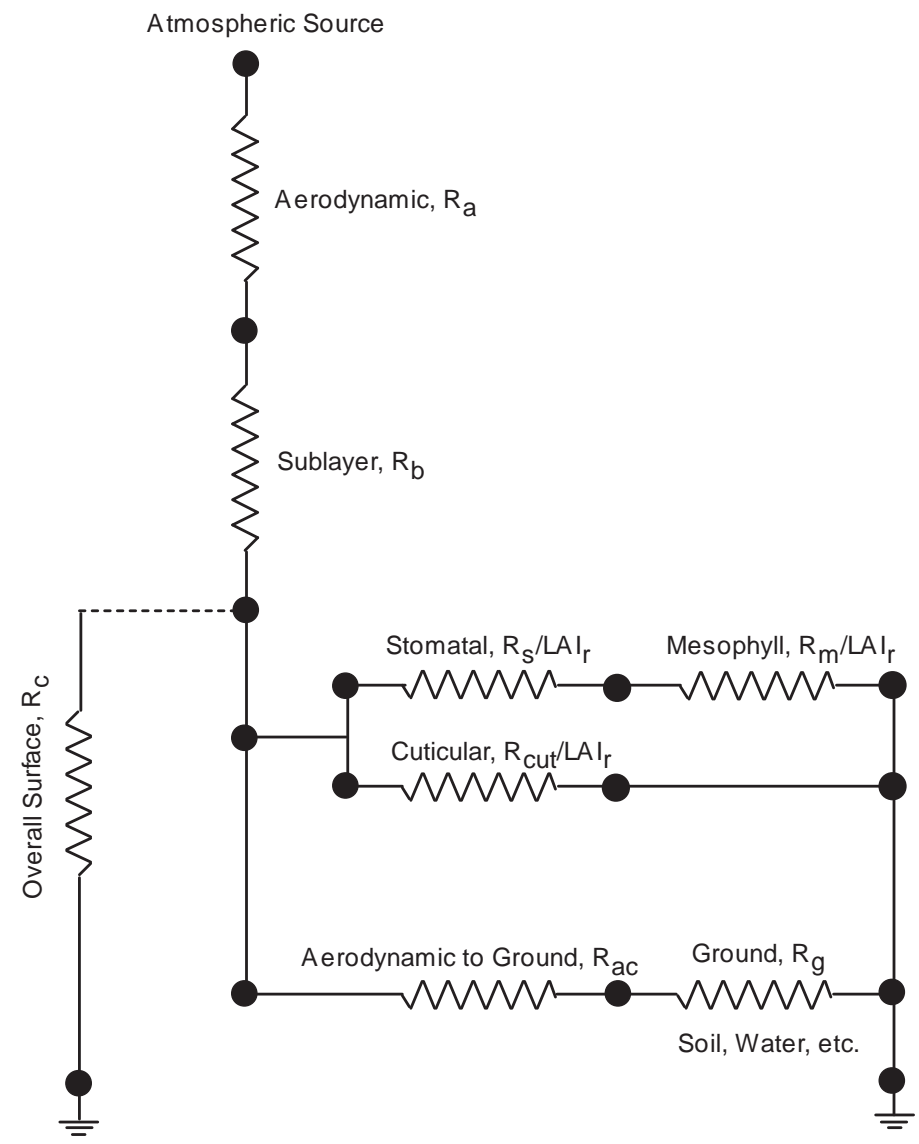

Figure 1. Scheme of major components of resistances used for gaseous dry deposition.

Equation 5 and Figure 1 are based in part on procedures used in RADM, which describes two separate nonstomatal pathways for the upper and lower canopy. Here the two pathways have been consolidated into one pathway represented by the term, $R_{\text {cut }}$, which is dependent on the terms $\mathrm{R}_{\mathrm{CS}}$ for $\mathrm{SO}_{2}$ and $\mathrm{R}_{\mathrm{CO}}$ for $\mathrm{O}_{3}\left(\mathrm{R}_{\mathrm{clS}}\right.$ and $\mathrm{R}_{\mathrm{clO}}$ in $\mathrm{RADM}$ notation). This procedure simplifies the table of input resistances and eliminates the need to consider an in-canopy aerodynamic resistance to transport to leaves the lower canopy.

Table 3, derived in part from Wesely's (1989) Table 1, shows the fixed or computationally initial values of all of the bulk surface resistances included in equation (5). The term $R_{i}$ represents the minimum stomatal resistance and is used to estimate $R_{S} ; R_{c S}$ and $R_{c O}$ signify reference values for evaluating $R_{\text {cut }}$ for the substance of interest; and $R_{g S}$ and $R_{g O}$ are likewise used to find $\mathrm{R}_{\mathrm{g}}$. No attempt is made here to account explicitly for the fraction of area covered by vegetative canopies. Instead, we assume that this fraction is implicitly considered in the values shown in Table 3 . The values of $R_{\text {aci }}$ in Table 3 are appropriate for moderate wind speeds, when $u_{*}$ is approximately $0.3 \mathrm{~m} \mathrm{~s}^{-1}$. Estimates of $R_{a c}$ are made as the product of $0.3 u_{*}{ }^{-1}$ 
and $\mathrm{R}_{\mathrm{aci}}$, by following Erisman's (1994) approximation that $\mathrm{R}_{\mathrm{ac}}$ is inversely proportional to friction velocity $\mathrm{u}_{*}$. 
Table 3. Values of surface resistances $\left(\mathrm{s} \mathrm{m}^{-1}\right)$ for model inputs. Entries of "107" indicate extremely large resistances or no deposition pathway.

\begin{tabular}{llllllllll}
\hline & \multicolumn{10}{c}{ Land use type } \\
\cline { 2 - 9 } Resistance & 1 & 2 & 3 & 4 & 5 & 6 & 7 & 8 & 9
\end{tabular}

Seasonal category 1 , midsummer with lush vegetation

\begin{tabular}{lrrrrrrrrr}
\hline$R_{i}$ & $10^{7}$ & 60. & 120. & 100. & 200. & 150. & $10^{7}$ & $10^{7}$ & 80. \\
$R_{c S}$ & $10^{7}$ & 2000. & 2000. & 2000. & 2000. & 2000. & $10^{7}$ & $10^{7}$ & 2500. \\
$R_{c O}$ & $10^{7}$ & 1000. & 1000. & 1000. & 2000. & 2000. & $10^{7}$ & $10^{7}$ & 1000. \\
$R_{a c i}$ & 100. & 200. & 100. & 2000. & 100. & 1500. & 0. & 0. & 300. \\
RgS & 400. & 150. & 350. & 300. & 500. & 450. & 0. & $10^{7}$ & 0. \\
$R_{g O}$ & 300. & 150. & 200. & 200. & 300. & 300. & 2000. & 400. & 1000.
\end{tabular}

Seasonal category 2, autumn with unharvested cropland (default value: $F=0.5$ )

\begin{tabular}{lrrrrrrrrr}
\hline $\mathrm{Ri}_{\mathrm{r}}$ & $10^{7}$ & $10^{7}$ & $10^{7}$ & 350. & $10^{7}$ & 700. & $10^{7}$ & $10^{7}$ & $10^{7}$ \\
$\mathrm{R}_{\mathrm{CS}}$ & $10^{7}$ & 6500. & 6500. & 3000. & 2000. & 2000. & $10^{7}$ & $10^{7}$ & 6500. \\
$\mathrm{R}_{\mathrm{CO}}$ & $10^{7}$ & 400. & 300. & 500. & 600. & 1000. & $10^{7}$ & $10^{7}$ & 300. \\
$\mathrm{Raci}$ & 100. & 150. & 100. & 1700. & 100. & 1200. & 0. & 0. & 200. \\
$\mathrm{RgS}$ & 400. & 200. & 350. & 300. & 500. & 450. & 0. & 1000. & 0. \\
$\mathrm{R}_{\mathrm{gO}}$ & 300. & 150. & 200. & 200. & 300. & 300. & 2000. & 400. & 800.
\end{tabular}

Seasonal category 3 , late autumn after frost and harvest, or winter with no snow

\begin{tabular}{lrrrrrrrrr}
\hline$R_{i}$ & $10^{7}$. & $10^{7}$ & $10^{7}$ & 500. & $10^{7}$ & 1000. & $10^{7}$ & $10^{7}$ & $10^{7}$ \\
$\mathrm{R}_{\mathrm{CS}}$ & $10^{7}$. & $10^{7}$ & 9000. & 6000. & 2000. & 2000. & $10^{7}$ & $10^{7}$ & 9000. \\
$\mathrm{R}_{\mathrm{CO}}$ & $10^{7}$ & $10^{7}$ & 400. & 600. & 800. & 1600. & $10^{7}$ & $10^{7}$ & 800. \\
$\mathrm{R}_{\mathrm{aci}}$ & 100. & 0. & 100. & 1500. & 100. & 1000. & 0. & 0. & 100. \\
$\mathrm{RgS}$ & 400. & 150. & 350. & 300. & 500. & 450. & 0. & 0. & 1000. \\
$\mathrm{R}_{\mathrm{gO}}$ & 300. & 150. & 200. & 200. & 300. & 300. & 2000. & 400. & 1000.
\end{tabular}

Seasonal category 4, winter with snow on ground

$\begin{array}{lrrrrrrrrr}\mathrm{R}_{\mathrm{i}} & 10^{7} & 10^{7} & 10^{7} & 800 . & 10^{7} & 1600 . & 10^{7} & 10^{7} & 10^{7} \\ \mathrm{R}_{\mathrm{CS}} & 10^{7} & 10^{7} & 10^{7} & 400 . & 10^{7} & 800 . & 10^{7} & 10^{7} & 9000 . \\ \mathrm{R}_{\mathrm{CO}} & 10^{7} & 2000 . & 1000 . & 600 . & 2000 . & 1200 . & 10^{7} & 10^{7} & 800 . \\ \mathrm{R} & 100 . & 0 . & 10 . & 1500 . & 100 . & 1000 . & 0 . & 0 . & 50 . \\ \mathrm{R}_{\mathrm{gS}} & 100 . & 100 . & 100 . & 100 . & 200 . & 200 . & 0 . & 1000 . & 100 . \\ \mathrm{R}_{\mathrm{gO}} & 600 . & 3500 . & 3500 . & 3500 . & 500 . & 500 . . & 2000 . & 400 . & 3500 .\end{array}$

Seasonal category 5 , transitional spring with partial green coverage or short annuals

(default value: $\mathrm{F}=0.25$ )

\begin{tabular}{lrrrrrrrrr}
$\mathrm{R}_{\mathrm{i}}$ & $10^{7}$ & 100. & 120. & 100. & 200. & 150. & $10^{7}$ & $10^{7}$ & 80. \\
$\mathrm{R}_{\mathrm{CS}}$ & $10^{7}$ & 2000. & 2000. & 1500. & 2000. & 2000. & $10^{7}$ & $10^{7}$ & 2000. \\
$\mathrm{R}_{\mathrm{cO}}$ & $10^{7}$ & 1000. & 250. & 350. & 500. & 700. & $10^{7}$ & $10^{7}$ & 300. \\
$\mathrm{R}_{\mathrm{aci}}$ & 100. & 50. & 80. & 1500. & 100. & 1000. & 0. & 0. & 200. \\
$\mathrm{R}_{\mathrm{gS}}$ & 500. & 150. & 350. & 300. & 500. & 450. & 0. & 1000. & 0. \\
$\mathrm{R} g \mathrm{O}$ & 300. & 150. & 200. & 200. & 300. & 300. & 2000. & 400. & 1000. \\
\hline
\end{tabular}




\subsubsection{Scaling with Leaf Area Index}

The scaling of stomatal and cuticular resistances with total leaf area index LAI is a common practice. Recent work has shown that linear scaling is a good approximation for forests throughout the growing season (e.g., Sakai et al., 1997), but some evaporation studies have shown that air-surface exchange by agricultural crops and grasses tends to increase at rates less than linear as the maximum LAI for the individual vegetative stand is approached (e.g., Rosenberg et al., 1983). Large-scale models sometimes have used nonlinear scaling throughout the domain (e.g., Abramopoulos et al., 1988). Air-land surface exchange models typically scale the bulk surface conductance $G_{S}=L_{A} I_{r}\left(R_{S}+R_{m}\right)^{-1}$ as directly proportional to the simple ratio SR of remotely sensed surface reflectance of near-infrared radiation to the reflectance of red radiation (e.g., Sellers et al., 1997); the SR increases nearly linearly with small values of LAI but less rapidly at large LAI. The types of modeling described by Sellers et al. (1997) incorporate a mathematical description of the change of LAI with height in a vegetated canopy and model the absorption of photosynthetically active radiation and stomatal response with height in the canopy. This approach, however, requires fairly exhaustive parameterization of canopy physical and physiological characteristics that are specific to various plant species. Because the sitespecific information needed to implement such an approach in ISC is usually not available, a simpler method is recommended here. Only a relative value of LAI and the sets of fixed or initial foliage resistances in Table 3 are used. Scaling is done with a relative LAI, or LAI . For wooded areas (land use types 4 and 6 in Table 1), we assume the following relationship:

$$
\mathrm{LAI}_{\mathrm{r}}=\mathrm{F} .
$$

For all other vegetative surfaces, we assume the following:

$$
\mathrm{LAI}_{\mathrm{r}}=\mathrm{F}^{0.5} \text {. }
$$

Here $\mathrm{F}$ is the fraction of maximum green LAI. To determine the value of $\mathrm{F}$, one of the seasonal categories listed in Table 2 must be specified by the ISC model user. The value of F used to determine $\mathrm{LAI}_{\mathrm{r}}$ in equation (5) can be between 0 and 1 but has no impact in the calculations for which values of $10^{7}$ are listed in Table 3. For seasonal categories 2 and 5, the value of $F$ (requested from the user) could be a rough estimate based on a visual inspection at the specific site. Otherwise, the default values of 0.5 for category 2 and 0.25 for category 5 are applied. For the other categories, the value of $\mathrm{F}$ is always set to 1.0.

\subsubsection{Stomatal Resistance}

The bulk stomatal resistance $\mathrm{R}_{\mathrm{S}}$ is found from estimates of the minimum stomatal resistance $R_{i}$ for various types of vegetation by scaling $R_{i}$ with multiplicative factors $f_{1}, f_{2}, f_{3}$, and $\mathrm{f}_{4}$ for environmental conditions, in much the same way as is often done for individual leaves. The primary environmental conditions considered involve solar irradiance, soil moisture content, humidity, and temperature. In addition, the stomatal resistance is adjusted with the ratio of the diffusivity of the gas of interest to that for water vapor, per standard practice: 


$$
R_{S}=R_{i}\left(D_{v} / D_{a}\right)\left(f_{1} f_{2} f_{3} f_{4}\right)^{-1}
$$

Here $R_{i}$ is obtained from Table $3, D_{v}$ is the diffusivity of water vapor in air (set to $0.219 \times 10^{-4} \mathrm{~m}^{2} \mathrm{~s}^{-1}$ ), and $\mathrm{D}_{\mathrm{a}}$ is the diffusivity of the gas of interest in air, estimated and provided in tabular form as described in Section 6.1 of this document.

\subsubsection{Solar Irradiance}

For solar irradiance, the function $\mathrm{f}_{1}$ is based on the Models- 3 dry deposition model formula and has an effect similar to that of the formula used in the RADM module:

$$
f_{1}=\left(G / G_{r}+0.01\right)\left(G / G_{r}+1\right)^{-1} .
$$

Here $\mathrm{G}$ is the solar irradiance; the term $\mathrm{G}_{\mathrm{r}}$ represents a reference value, which is set equal to $30 \mathrm{~W} \mathrm{~m}^{-2}$ for forests and to $100 \mathrm{~W} \mathrm{~m}^{-2}$ for crops.

\subsubsection{Soil Moisture}

To account for the effects of soil moisture stress, the Models-3 dry deposition module takes a fairly detailed approach based on soil moisture information produced by a mesoscale meteorological model. The RADM module did not consider soil moisture stress directly. Here, values between 0 and 1 are assigned to the relative available, or extractable, soil moisture $\theta_{\mathrm{r}}$, which is inferred from precipitation data and evaporation estimates. To relate the soil moisture stress factor $f_{2}$ to $\theta_{\mathrm{r}}$, nonlinear relationships such as a simple exponential function (e.g., Kim and Verma, 1991) and logistic curves (Byun et al., 1999) can be used. Nonlinear relationships are often favored because they can describe fairly well the soil moisture retention curve (the soil water potential versus water content). Tests conducted by Betts et al. (1997) and other research, however, indicate that a simple linear relationship such as $\mathrm{f}_{2}=\theta_{\mathrm{r}}$ appears to provide better estimates of $\mathrm{f}_{2}$ in modeling for some situations; this approach is recommended for ISC.

To obtain an estimate of $\theta_{\mathrm{r}}$, some rough approximations are made for soil moisture amount available to vegetation as affected by precipitation and evaporation. We compute the water amount $\mathrm{w}_{\mathrm{hr}}(\mathrm{mm})$ in the upper layer of soil for the current hour $(\mathrm{hr})$ on the basis of water amount $\mathrm{w}_{\mathrm{hr}-1}$ for the preceding hour, the precipitation (water equivalent) rate $\mathrm{P}_{\mathrm{hr}-1}\left(\mathrm{~mm} \mathrm{hr}^{-1}\right)$ during the preceding hour, and an hourly estimate of evaporation. The maximum available water amount in the soil is assumed to be $200 \mathrm{~mm}$ of water; amounts ranging between $100 \mathrm{~mm}$ and $200 \mathrm{~mm}$ in the upper layer of $1 \mathrm{~m}$ or so of soil are typically available for evaporation. For minimal soil moisture stress, the average evaporation rate is assumed to be $12 \mathrm{~mm}$ per day for ambient air temperatures near $25^{\circ} \mathrm{C}$; for other temperatures, the average evaporation rate is estimated by multiplying by the ratio of the saturation vapor pressure $e_{S}(T)$ in ambient air for the previous hour to the saturation vapor pressure $\mathrm{e}_{\mathrm{S}}(25)$ at $25^{\circ} \mathrm{C}$. This scaling provides roughly a doubling of evaporation rate for each increase of $10^{\circ} \mathrm{C}$ in air temperature, for unstressed conditions. Such approximations can result in uncertainties in evaporation estimates at least as large as $\pm 25 \%$. These assumptions and approximations are derived from various accounts of the 
behavior and properties of soils and evaporation (e.g., Buckman and Brady, 1960; Rutter, 1975; Brutsaert, 1982; Oke, 1987; Hillel, 1998). The resulting equation is

$$
\mathrm{w}_{\mathrm{hr}}=\mathrm{w}_{\mathrm{hr}-1}+\mathrm{P}_{\mathrm{hr}-1}-0.5 \mathrm{f}_{2} \mathrm{e}_{\mathrm{s}}(\mathrm{T}) / \mathrm{e}_{\mathrm{s}}(25)
$$

Then $\mathrm{f}_{2}$ is estimated from the relationship $\theta_{\mathrm{r}}=\mathrm{w}_{\mathrm{hr}-1} / 200$. For initial conditions, values of $\mathrm{w}_{\mathrm{hr}-1}=180 \mathrm{~mm}$ and $\theta_{\mathrm{r}}=0.9$ can be assumed. The maximum value allowed for water amount is $200 \mathrm{~mm}$. Although this approach addresses hourly values, estimates of evaporation or soil moisture content probably should not be examined for accuracy for time periods shorter than one day; one week or longer would be preferred. This approach is unlikely to give excellent hydrological estimates of soil water content but is more realistic than assuming that the soil water content does not change with time.

\subsubsection{Humidity}

For the effects of vapor pressure deficit or relative humidity $\mathrm{RH}$, a linear dependence on $\mathrm{RH}$ at the surface of leaves would provide a state-of-the-science approach like the one used in the Models-3 dry deposition model (Pleim, 1999; Byun et al., 1999). Computation of this surface RH requires estimates of ambient humidity, ambient temperature, and surface sensible and latent heat fluxes. The ISC meteorological preprocessor, however, provides estimates of surface fluxes that are probably not adequately representative of the local surface to compute surface RH with sufficient accuracy. The fairly common, simpler approach of involving the water vapor deficit is recommended for ISC:

$$
\mathrm{f}_{3}=(1+\alpha \delta \mathrm{e})^{-1} \text {, }
$$

where $\delta$ e is the vapor pressure deficit, and $\alpha$ represents a coefficient equal to about $0.1 \mathrm{kPa}^{-1}$ for ambient air about $2 \mathrm{~m}$ above the surface (e.g., Kim and Verma, 1991).

\subsubsection{Temperature}

To describe the effects of temperature on stomatal resistance, the function $\mathrm{f}_{4}$ can be selected from several formulas that are available in the scientific literature. In comparison to the RADM module formula, the formula used in Models-3 produces smaller effects of the temperature-induced reduction of stomatal conductance at elevated temperatures. Relatively advanced formulations gain their advantage in accuracy in part by using an estimate of the surface temperature of leaves, but the estimates of surface temperature that could be derived from ISC parameter values are unlikely to be sufficiently accurate for this purpose. Furthermore, this environmental factor varies with plant species. Here, a relatively simple approach will suffice (Noihan and Planton, 1989):

$$
\mathrm{f}_{4}=1-0.0016\left(298.0-\mathrm{T}_{\mathrm{a}}\right)^{2},
$$

where $\mathrm{T}_{\mathrm{a}}$ is ambient air temperature in Kelvin at a height of about $2 \mathrm{~m}$ above the ground. 


\subsubsection{Mesophyll, Cuticular, and Ground Resistances}

\subsubsection{Solubility in Water}

The effects of solubility and aqueous dissociation on mesophyll, cuticular, and ground resistances are keyed to the corresponding resistances for $\mathrm{SO}_{2}$, which are then adjusted by multiplication by the ratio of effective Henry's Law values. Such an approach is used in the RADM module and is adequate for most substances. The cuticular and ground resistances appear to be overestimated by the RADM routines, however, for substances like $\mathrm{H}_{2} \mathrm{O}_{2}$ that are both moderately soluble in water and strong oxidizing agents (e.g., Hall et al., 1999). Substances with such properties are not on the list of HAPs of concern here, but a slight modification nevertheless is made to some of the formulations by adding an additional term involving the value of the Henry's Law constant and the measure of reactivity $\left(f_{\mathrm{o}}\right)$. The values for the Henry's Law constants $(\mathrm{H})$ are provided in tabular form as described in Section 6.1 of this document in units of Pascals-cubic meters per mole $\left(\mathrm{Pa} \mathrm{m}^{3} \mathrm{~mol}^{-1}\right)$. Because these units are different from the units of atmospheres-liters per mole ( $\mathrm{atm} \mathrm{M}^{-1}$ ) used in the RADM module, the formulations are altered correspondingly, as shown in Section 2.2.3.6.

\subsubsection{Reactivity}

Although no definition of reactivity is well established for dry deposition parameterizations, the primary factor for uptake by natural surfaces is the strength of the substance as an oxidizing agent. Ozone provides a reference scaling quantity. The primary measures of reactivity involve half reduction-oxidation reactions in neutral aqueous solutions and overall second-order reaction rates with $S(I V)$, which is sulfur in oxidation state +4 . If the practices in the RADM dry deposition module were followed, a reactivity factor $f_{\mathrm{o}}$ would be assigned values of $0,0.1$, or 1 . However, the values of $f_{0}$ are set to zero for all of the substances addressed here (except for two inorganic gases considered in Section 6.5), because of their very weak oxidizing capabilities.

A facet of reactivity not explicitly considered here is the effects of variable surface $\mathrm{pH}$. The deposition of acidic gases can be reduced when the $\mathrm{pH}$ of surface materials decreases. For example, the uptake of $\mathrm{SO}_{2}$ by bare soil is partially controlled by soil $\mathrm{pH}$ (e.g., Baldocchi, 1993).

\subsubsection{Lipid Solubility}

Studies have shown that lipid solubility of organics is associated with a limited uptake term that can be modeled if sufficient information is available on the chemical characteristics of the substances and the cuticular resistance to uptake for each substance (e.g., Deinum et al., 1995). As described in Section 6.4 of this document, the cuticular resistance $r_{c l}$ for individual leaves to uptake by lipids can be related theoretically to the octanol-water partition coefficient for organics through the use of baseline data on cuticle membrane studies in the laboratory. The values for $r_{\mathrm{cl}}$ are provided in tabular form as described in Section 6.4. To apply this approach, 
the resistances for individual leaves are scaled to represent whole plant canopies. That is, the bulk canopy resistance $\mathrm{R}_{\mathrm{cl}}$ to uptake associated with lipid solubility is computed as

$$
\mathrm{R}_{\mathrm{cl}}=\mathrm{r}_{\mathrm{cl}}\left(\mathrm{LAI}_{\mathrm{r}} \mathrm{S}\right)^{-1}
$$

where $\mathrm{S}$ is a scaling factor based nonlinearly on the maximum two-sided leaf area index likely for various land use categories. Values of $\mathrm{S}$ are tabulated in Table 4.

A difficulty with this method of estimating $r_{\mathrm{cl}}$ and $\mathrm{R}_{\mathrm{cl}}$ is that the leaf cuticles can have limited capacity to take up organics, especially if the substances are not chemically reactive with leaf materials. If high ambient concentrations occur over the same area for an extended period of time, the leaf could become saturated, driving $\mathrm{R}_{\mathrm{cl}}$ to considerably larger values than those estimated with equation (12). To avoid highly unrealistic situations, a minimum value of $\mathrm{R}_{\mathrm{cl}}=$ $100 \mathrm{~s} \mathrm{~m}^{-1}$ is assumed for all substances.

\subsubsection{Surface Temperature}

Subfreezing temperatures tend to inhibit the ability of surfaces to take up substances that are otherwise quite reactive or soluble. The simple parameterization in the RADM module can be applied to describe this effect for all cuticular and ground surfaces by adding the value of $1000 \mathrm{e}^{\left(-\mathrm{T}_{\mathrm{a}}+269.2\right)}$, expressed in units of seconds per meter, to all resistances for all such external surfaces.

Table 4. Values of the factor $S$ to scale leaf resistance $r_{\mathrm{cl}}$ to uptake associated with lipid solubility, to estimate leaf resistance $R_{C l}$ for entire canopies.

\begin{tabular}{cc}
\hline Land use category & Factor \\
\hline & \\
1 & 0 \\
2 & 6 \\
3 & 5 \\
4 & 7 \\
5 & 3 \\
6 & 4 \\
7 & 8 \\
8 & 7 \\
9 & 0 \\
\end{tabular}




\subsubsection{Surface Wetness}

Experimental studies have shown that the approach used in the RADM dry deposition module for estimating resistances for surfaces wetted by dew and rain is not realistic for $\mathrm{O}_{3}$ and $\mathrm{SO}_{2}$. For $\mathrm{O}_{3}$, whose surface resistance is used as a reference resistance in evaluating the effects of reactivity on uptake of diverse substances, dew rarely inhibits removal and at times can increase it. A possible course of action might be to neglect any effect of dew on $\mathrm{O}_{3}$ surface resistance; a slightly more aggressive approach would be to assume that dew typically decreases surface resistance.

For rainfall, most of the studies during the past ten years or so indicate almost no effect on $\mathrm{O}_{3}$ removal by vegetation, at least during the daytime (e.g., Enders et al., 1992; Fuentes et al., 1992; Padro, 1994; Grantz et al., 1995). Rainfall might have an enhancing effect during the night. The recommended approach is to make no distinction between dew and rainfall and to reduce the leaf cuticular resistance by $25 \%$ when the surface is wetted.

The surface resistance for $\mathrm{SO}_{2}$ provides the reference resistance in evaluating the effects of water solubility on the uptake resistances of various substances. Studies in rural areas show that the surface resistance for vegetated surfaces is about $R_{c}=50 \mathrm{~s} \mathrm{~m}^{-1}$ for all surfaces wetted by either rain or dew (e.g., Fowler, 1978; Baldocchi, 1993; Erisman, 1994; Padro, 1994). In places where $\mathrm{NH}_{3}$ concentrations are high, the wetted surface resistances for $\mathrm{SO}_{2}$ might be near zero (Erisman and Wyers, 1993; Cape et al., 1998). However, for cases where rain is sufficiently acidic or saturated with the forms $\mathrm{SO}_{2}$ and $\mathrm{SO}^{2-}$ of $\mathrm{S}(\mathrm{IV})$, the surface resistance for $\mathrm{SO}_{2}$ might be increased when wetted by rain. The cases for high $\mathrm{NH}_{3}$ and $\mathrm{S}(\mathrm{IV})$ saturated rain can probably be ignored in the United States except very near $\mathrm{NH}_{3}$ and low-level $\mathrm{SO}_{2}$ sources. For a fully wetted surface, a value of $50 \mathrm{~s} \mathrm{~m}^{-1}$ is recommended for leaf cuticular and ground resistances.

The fraction of a surface that is wetted by rain or dew is difficult to estimate with models and observations except in extreme cases of wetness or dryness. The RADM module assumes only either wet or dry extremes, and Models-3 dry deposition algorithms assume that the fractional leaf area wetness is inferred from information provided by a mesoscale meteorological model. Although an intermediate or partially wetted state would be useful because of the possibility of long-term partial wetness, providing the input data to identify the case of intermediate wetness is probably impractical for ISC applications. The procedure here is that two states of wetness are provided as inputs: wet and dry. The resistances are estimated as follows:

$$
\begin{array}{ll}
\mathrm{R}_{\mathrm{cOw}}=0.75 \mathrm{R}_{\mathrm{cO}} & \text { for } \mathrm{O}_{3} \text {, wetted } \\
\mathrm{R}_{\mathrm{cSw}}=50 \mathrm{~s} \mathrm{~m}^{-1} & \text { for } \mathrm{SO}_{2} \text {, wetted } \\
\mathrm{R}_{\mathrm{gSw}}=50 \mathrm{~s} \mathrm{~m}^{-1} & \text { for } \mathrm{SO}_{2} \text {, wetted }
\end{array}
$$

No attempt is made here to take into account the effects of extremely wet soil conditions or water ponded on the ground. In such cases, the ground resistances would increase for $\mathrm{O}_{3}$ uptake and decrease for $\mathrm{SO}_{2}$ uptake. 
To estimate the occurrence of dew from local observations or calculations based on local observations, an adaptation of the scheme of Jannssen and Römer (1991) is followed. That is, a dew-wetted surface is assumed to exist when the following condition is met:

$$
\mathrm{u}_{*}<0.3(\delta \mathrm{q})^{-1},
$$

where $\delta q$ is the difference in specific humidity $\left(\mathrm{g} \mathrm{kg}^{-1}\right)$ calculated for ambient dewpoint temperature versus saturation at ambient air temperature, and $\mathrm{u}_{*}$ has units of $\mathrm{m} \mathrm{s}^{-1}$. The numerical coefficient 0.3 is provided by Jannssen and Römer's (1991) analysis for the case of the cloud cover fraction between $2 / 8$ and $6 / 8$ (25\% and $75 \%)$. To conform fully with the results of Jannssen and Römer, the coefficient 0.3 is replaced by 0.45 when the cloud fraction is less than $2 / 8$ and by 0.15 when the cloud fraction is greater than $6 / 8$.

To identify cases when a rain-wetted surface exists, the surface is assumed to be wet when precipitation occurs and for a period of $2 \mathrm{hr}$ thereafter. Hourly precipitation data is used for this purpose. The criterion for identifying a dew-wetted surface is applied simultaneously with this criterion for wetting by rain.

\subsubsection{Calculation of Mesophyll, Cuticular, and Ground Resistances}

The effects of water solubility, reactivity, and lipid solubility can be embodied in mesophyll, cuticular, and ground resistances as follows:

$$
\begin{aligned}
& \mathrm{R}_{\mathrm{m}}=\left(0.034 \mathrm{H}^{-1}+100 f_{\mathrm{o}}\right)^{-1}, \\
& \mathrm{R}_{\mathrm{cut}}=\left[10^{-3}\left(\mathrm{HR}_{\mathrm{cS}}\right)^{-1}+\left(f_{\mathrm{o}}+f_{\mathrm{o}}^{2} / \mathrm{H}\right) \mathrm{R}_{\mathrm{cO}}{ }^{-1}+\mathrm{R}_{\mathrm{cl}}{ }^{-1}\right]^{-1}, \\
& \mathrm{R}_{\mathrm{g}}=\left[10^{-3}\left(\mathrm{HR}_{\mathrm{gS}}\right)^{-1}+\left(f_{\mathrm{o}}+0.1 f_{\mathrm{o}}^{2} / \mathrm{H}\right) \mathrm{R}_{\mathrm{gO}} \mathrm{O}^{-1}\right]^{-1},
\end{aligned}
$$

The terms $\mathrm{R}_{\mathrm{cS}}, \mathrm{R}_{\mathrm{cO}}$, and $\mathrm{R}_{\mathrm{gS}}$ are to be replaced by the corresponding resistances given by equations (13-15) if the surface is wet. These three resistances, for both wet and dry surfaces, and the ground resistance $\mathrm{R}_{\mathrm{gO}}$ must all be modified individually by addition of the term $1000 \mathrm{e}^{\left(-\mathrm{T}_{\mathrm{a}}+269.2\right)}$. 


\section{Dry Deposition of Particles}

The deposition velocity $\mathrm{V}_{\mathrm{dp}}$ for particles can be expressed as follows (e.g., Slinn and Slinn, 1980; Pleim et al., 1984; Hicks, 1986):

$$
V_{d p}=\left(R_{a}+R_{p}+R_{a} R_{p} V_{g}\right)^{-1}+V_{g} .
$$

Here $\mathrm{R}_{\mathrm{a}}$ is the aerodynamic resistance $\left(\mathrm{s} \mathrm{m}^{-1}\right.$ ) given by equation (2), $\mathrm{R}_{\mathrm{p}}$ represents the resistance $\left(\mathrm{s} \mathrm{m}^{-1}\right)$ to particle deposition in the quasilaminar sublayer enveloping surface elements, and $\mathrm{V}_{\mathrm{g}}$ represents the gravitational settling velocity $\left(\mathrm{m} \mathrm{s}^{-1}\right)$. This method assumes that individual particles do not change size with height as a result of processes associated with humidity changes, coagulation, etc. When this approach is applied to environmental aerosols, the particle size distribution is assumed not to change with height, for example because of gravitational settling or impaction selectively removing particles in certain size ranges. Although these assumptions are not always valid, the uncertainties that result appear to be small relative to other uncertainties, especially those resulting from assumptions or estimates about the particle size distribution. Overall, equation (20) indicates a resistance scheme somewhat analogous to equation (1) but with $R_{b}$ replaced by $R_{p}$, the assumption that $R_{c}$ for transport to the inner and outer surfaces of surface elements is negligible or incorporated into $R_{p}$, and $V_{g}$ representing a deposition pathway in parallel with $\left(\mathrm{R}_{\mathrm{a}}+\mathrm{R}_{\mathrm{p}}\right)^{-1}$.

The components $R_{p}$ and $V_{g}$ are functions of particle size, and equation (20) would ideally be evaluated for size intervals matching measurements of the particle size distribution. The recommended approach for ISC applications is to have the model user select one of two methods for modeling particle dry deposition velocities. Because of the major contribution of gravitational settling of large particles to dry deposition velocity, the method (here, termed Method 1) described by Schwede and Paumier (1997) should be chosen when a considerable fraction, say 0.10 or more, of the total particulate mass of the substance or material of interest is in particles with a diameter of $10 \mu \mathrm{m}$ and larger. The particle size distribution must be known reasonably well from measurements of ambient air for emission sources very similar to the source being considered. For example, fugitive dust emissions from mining operations would require the use of Method 1 (Environmental Protection Agency, 1994). Method 2 is a somewhat simplified approach for the case when the particle size distribution is not well known, except that past measurements of the particulate substance have indicated that only a small fraction of mass is in particles $10 \mu \mathrm{m}$ and larger.

The ISC formulations described by Schwede and Paumier (1997) constitute Method 1, but one equation should be changed. That is, their equation (5) needs to be modified to take into account more thoroughly the effects of gusty winds during strongly unstable atmospheric conditions on the inertial processes that affect deposition. Atkinson et al. (1997) noted that the overestimates seen in concentrations simulated with ISC, in comparison to measured concentrations of $\mathrm{ZnS}$ particle tracers in an experiment described by Doran and Horst (1985), suggested that the deposition velocity was underestimated in unstable conditions. To address this concern, the following equation derived by Binkowski and Shankar (1995), combining theoretical expressions of Slinn (1982) with empirical observations by Wesely et al. (1985), should replace Schwede and Paumier's (1977) equation (5): 


$$
\mathrm{R}_{\mathrm{p} i}=\left[\left(\mathrm{Sc}_{i}^{-2 / 3}+10^{-3 / \mathrm{St}_{i}}\right)\left(1+0.24 \mathrm{w}_{*}{ }^{2} / \mathrm{u}_{*}^{2}\right) \mathrm{u}_{*}\right]^{-1}
$$

The change is incorporation of the factor $\left(1+0.24 \mathrm{w}_{*}{ }^{2} / \mathrm{u}_{*}^{2}\right)$. Here Sc is the Schmidt number and St is the Stokes number for particles in size bin $i$, and $\mathrm{w}_{*}$ is the convective scale velocity, derived from atmospheric stability parameters and the depth of the planetary boundary layer. A version of equation (20) can then be applied:

$$
\mathrm{V}_{\mathrm{dp} i}=\left(\mathrm{R}_{\mathrm{a}}+\mathrm{R}_{\mathrm{p} i}+\mathrm{R}_{\mathrm{a}} \mathrm{R}_{\mathrm{pi}} \mathrm{V}_{\mathrm{g} i}\right)^{-1}+\mathrm{V}_{\mathrm{g} i}
$$

where $\mathrm{V}_{\mathrm{gi}}$ is the gravitational settling velocity for particles in bin $i$.

Method 2, for the situation when coarse particles do not contribute significantly to dry deposition, was developed because measurements of particle size distributions are not always available, and no widely accepted set of exhaustive information exists on particle size distributions for the HAPs emitted from all of sources types of interest. Furthermore, "stack" emissions inventories for sources involving combustion typically do not provide adequate descriptions of particle size distributions in the air beyond extremely short distances from the sources, because of the effects of rapid processes involving particle nucleation, coagulation, and chemical reactions. Method 2 relies on reports in the scientific literature on particle measurements made in urban and industrial areas. Only two size categories were considered: a fine mode for particles smaller than about $2.5 \mu \mathrm{m}$ in diameter and a coarse mode for larger particles up to $10 \mu \mathrm{m}$ in diameter. No attempt is made to associate the size information with specific types of sources. Appendix B summarizes the results found in the scientific literature on the relative amount of mass typically found in the two size categories.

For the fine mode, the parameterization provided by Wesely et al. (1985) is applied:

$$
\begin{array}{ll}
\mathrm{R}_{\mathrm{p}}=500 \mathrm{u}_{*}^{-1} & \text { for } \mathrm{L} \geq 0, \\
\mathrm{R}_{\mathrm{p}}=500 \mathrm{u}_{*}{ }^{-1}[1+(-300 / \mathrm{L})]^{-1} & \text { for } \mathrm{L}<0 .
\end{array}
$$

Here, as in equation (2), $\mathrm{u}_{*}$ is the friction velocity, and $\mathrm{L}$ is the Monin-Obukhov length scale. Equation (23) is based on observations of sulfate particle deposition, so its use for the particulate HAP of interest effectively assumes that the fine mode has a particle size distribution similar to that for sulfate particles.

For the coarse mode in Method 2, we set $\mathrm{V}_{\mathrm{g}}$ equal to $0.02 \mathrm{~m} \mathrm{~s}^{-1}$, a representative value for particles with diameters of 5-7 $\mu \mathrm{m}$ (e.g., Slinn, 1977). The very coarse mode of particles larger than $10 \mu \mathrm{m}$ in diameter is not considered, although such large sizes might be important for a few of the particulate components listed in Appendix B, specifically for dust sources of beryllium associated with mining and granular sources of calcium cyanamide and cyanide compounds. 
To compute the dry deposition velocity of a particulate substance by using Method 2, equation (20) is applied twice, once for the fine mode with $\mathrm{V}_{\mathrm{g}}$ set to zero and once for the coarse mode with the representative value of $\mathrm{V}_{\mathrm{g}}=0.02 \mathrm{~m} \mathrm{~s}^{-1}$ :

$$
\begin{aligned}
& \mathrm{V}_{\mathrm{dpf}}=\left(\mathrm{R}_{\mathrm{a}}+\mathrm{R}_{\mathrm{p}}\right)^{-1}, \\
& \mathrm{~V}_{\mathrm{dpc}}=\left(\mathrm{R}_{\mathrm{a}}+\mathrm{R}_{\mathrm{p}}+0.02 \mathrm{R}_{\mathrm{a}} \mathrm{R}_{\mathrm{p}}\right)^{-1}+0.02 .
\end{aligned}
$$

The overall particle deposition velocity is then found as the weighted average of fine-mode deposition velocity $\mathrm{V}_{\mathrm{dpf}}$ and the coarse-mode deposition velocity $\mathrm{V}_{\mathrm{dpc}}$ :

$$
\mathrm{V}_{\mathrm{dp}}=x \mathrm{~V}_{\mathrm{dpf}}+(1-x) \mathrm{V}_{\mathrm{dpc}}
$$

Here, $x$ is the fraction given in Appendix B as percentages of the mass in the fine mode.

A variation of Methods 1 and 2 could be applied by describing two or three modes of particle size ranges in terms of log-normal distributions (e.g., Seinfeld and Pandis, 1998). Binkowski and Shankar (1995) give the equations to describe deposition velocity according to particle size modes much more rigorously than does Method 2. This more sophisticated approach is used in Models-3 for regional-scale applications. The parameters needed to describe each mode are the geometric mean size and the logarithmic standard deviation of the sizes. The values of these two parameters vary greatly, however, with time and from location to location. Thus, this approach cannot be applied readily to offer significant improvements in the accuracy of particle dry deposition velocity simulations with ISC. 


\section{Wet Deposition of Gases}

The procedure recommended for ISC to describe wet deposition rates of gases by rain relies on a washout ratio $\mathrm{W}_{\mathrm{g}}$ estimated as the reciprocal of the dimensionless Henry's Law constant (Slinn et al., 1978; Scott, 1981). This relationship between $\mathrm{W}_{\mathrm{g}}$ and $\mathrm{H}$ is most appropriate for chemical equilibrium conditions in a well-mixed planetary boundary layer. In terms of measurable quantities, $\mathrm{W}_{\mathrm{g}}$ is the ratio of the concentration of the gas in precipitation to the concentration in air. Use of $\mathrm{W}_{\mathrm{g}}$ is preferred over a scavenging ratio of the local removal rate to the concentration of pollutant in the local air, because ratios are usually described in the scientific literature as including the process of rainout in clouds; this often is not appropriate for short-range dispersion, where the plume is typically below cloud level.

For gas removal by snow, adsorption of the chemical in the thin film of liquid water that exists on the surface of ice crystals, which is similar to scavenging of substances by cloud or fog droplets, can produce washout ratios greater than those predicted by Henry's Law (Hoff et al., 1995). Results from field studies, however, have been inconclusive. For example, some chemical compound classes (e.g., polynuclear aromatic hydrocarbons [PAHs]) exhibit measured gas washout ratios for snow that are greater than those predicted by $\mathrm{H}$, while other classes of organics (e.g., polychlorinated byphenyls [PCBs]) exhibit ratios less (Franz and Eisenreich, 1998). Here, values of $\mathrm{W}_{\mathrm{g}}$ for the organic HAPs are assumed to be the same for snow as for rain.

Values of $\mathrm{W}_{\mathrm{g}}$ are calculated as $\mathrm{RT}_{\mathrm{a}} / \mathrm{H}$, where $\mathrm{R}$ is the ideal gas law constant (8.314 Pa m mol-1 $^{-1}$ ), and $\mathrm{T}_{\mathrm{a}}$ is the absolute temperature $(\mathrm{K})$. In this scheme, $\mathrm{W}_{\mathrm{g}}{ }^{-1}$ represents a unitless version of Henry's Law constant. Because the unit of volume addressed by $\mathrm{RT}_{\mathrm{a}}$ in the ideal gas law constant refers to the gaseous phase and the unit of volume of the Henry's Law constant $\mathrm{H}$ refers to the aqueous phase, the units for $\mathrm{W}_{\mathrm{g}}$ can be interpreted as being cubic meters of air per cubic meter of water. Thus, multiplying the vapor-phase concentration of the chemical in units of mass per unit volume by $\mathrm{W}_{\mathrm{g}}$ gives the concentration of the chemical in precipitation in units of mass per unit volume. The wet deposition rate is calculated as follows:

$$
\mathrm{F}_{\mathrm{g}}=10^{-3} \rho_{\mathrm{g}} \mathrm{W}_{\mathrm{g}} r
$$

where $\quad \mathrm{F}_{\mathrm{g}} \quad=$ flux of gas by wet deposition $\left(\mu \mathrm{g} \mathrm{m}^{-2} \mathrm{hr}^{-1}\right)$,

$\mathrm{W}_{\mathrm{g}}=$ gas washout ratio, equal to $\mathrm{RT}_{\mathrm{a}} / \mathrm{H}$ (unitless),

$\mathrm{R}=$ ideal gas law constant $\left(8.314 \mathrm{~Pa} \mathrm{~m}^{3} \mathrm{~mol}^{-1} \mathrm{~K}^{-1}\right)$,

$\mathrm{T}_{\mathrm{a}}=$ absolute air temperature $(\mathrm{K})$,

$\mathrm{H}=$ Henry's Law constant $\left(\mathrm{Pa} \mathrm{m}^{3} \mathrm{~mol}^{-1}\right)$,

$\rho_{\mathrm{g}} \quad=$ average column concentration of gaseous HAP in air $\left(\mu \mathrm{g} \mathrm{m}^{-3}\right)$,

$r=$ water or water-equivalent precipitation rate $\left(\mathrm{mm} \mathrm{hr}^{-1}\right)$.

Values of $\mathrm{H}$ and of $\mathrm{W}_{\mathrm{g}}$ for a temperature of $\mathrm{T}_{\mathrm{a}}=298.2 \mathrm{~K}$ are tabulated in Appendix $\mathrm{D}$.

To implement this approach with the existing ISC algorithms, estimates of $\rho_{\mathrm{g}}$ can be found from the following formula: 


$$
\rho_{\mathrm{g}}=\mathrm{z}_{\mathrm{t}}^{-1} \int_{0}^{\mathrm{z}_{\mathrm{t}}} \chi(\mathrm{x}, \mathrm{y}, \mathrm{z}) \mathrm{dz}
$$

where $\chi$ is the local concentration $\left(\mu \mathrm{g} \mathrm{m}^{-3}\right)$ in the plume at coordinate $(\mathrm{x}, \mathrm{y}, \mathrm{z})$, and $\mathrm{z}_{\mathrm{t}}(\mathrm{m})$ is the height of the top of the plume. Use of $\rho_{\mathrm{g}}$ from equation (26) in equation (25) effectively results in the assumption that $\chi$ is constant with height beneath the top of the plume, which is usually incorrect before precipitation. If the local equilibrium implied by the use of $\mathrm{H}$ to determine gas washout is achieved, however, the processes of uptake and outgassing from the precipitation tends to redistribute the gas vertically, making the value of $\chi$ less variable with height.

Application of equation (25) for substances with small values of $\mathrm{H}$ can produce large values of $F_{g}$, commensurate with rapid washout. The modeled values of must then be reduced along the trajectory of a plume as it is depleted. For gaseous wet deposition, the desired result regarding $\mathrm{F}_{\mathrm{g}}$ can be achieved by multiplying it by the wet depletion factor, as is currently done in ISC. That is, $F_{g}$ is multiplied by the fraction of material that remains in the plume as a function of downwind distance or equivalent travel time from the source. The wet depletion factor can be computed by following existing ISC practices as the term $\mathrm{e}^{-\Lambda t}$, where $t$ is plume travel time and $\Lambda$ is the scavenging ratio, found as

$$
\Lambda=a \mathrm{~W}_{\mathrm{g}} r \mathrm{z}_{\mathrm{t}}^{-1}
$$

where $a=2.77 \times 10^{-7}$ is a conversion factor to ensure that $\Lambda$ is expressed in units of $\mathrm{s}^{-1}$. The value of $z_{t}$ used in equation (27) tends to increase as a function of time $t$, causing an underestimate of the wet depletion factor. The amount of this underestimate is assumed to be negligible.

For emissions at the surface, the vertical extent of a plume can be very small near the source, resulting in large values of $\rho_{\mathrm{g}}$ found via equation (26). Such a situation could produce estimates of wet deposition rate $\mathrm{F}_{\mathrm{g}}$ that are excessively large because local chemical equilibrium is not achieved instantaneously as the precipitation falls through a thin plume. To overcome this difficulty, a minimum precipitation fall distance sufficient to achieve local equilibrium should be used for $z_{t}$ in equations (26) and (27). We assume that a minimum value of $z_{t}=100 \mathrm{~m}$ is appropriate for wet deposition of gases.

Rapid washout can also affect estimates of dry deposition. The simulated concentrations $\chi$ used to estimate dry deposition near the surface can be reduced by assuming that the wet depletion factor is constant with height, making it possible to reduce $\chi$ or the dry deposition rate directly through multiplication by the wet depletion factor. 


\section{Wet Deposition of Particles}

The particle-phase washout ratio $\mathrm{W}_{\mathrm{p}}$ provides the means to compute the wet deposition rate of particulate substances:

$$
\mathrm{F}_{\mathrm{p}}=10^{-3} \rho_{\mathrm{p}} \mathrm{W}_{\mathrm{p}} r
$$

where $\quad \mathrm{F}_{\mathrm{p}}=$ flux of particulate material by wet deposition $\left(\mu \mathrm{g} \mathrm{m}^{-2} \mathrm{hr}^{-1}\right)$,

$\mathrm{W}_{\mathrm{p}}=$ particle washout ratio (unitless),

$\rho_{\mathrm{p}} \quad=$ average column concentration of particulate HAP in air $\left(\mu \mathrm{g} \mathrm{m}^{-3}\right)$,

$r \quad=$ water or water-equivalent precipitation rate $\left(\mathrm{mm} \mathrm{hr}^{-1}\right)$.

The reasons for selecting $\mathrm{W}_{\mathrm{p}}$ as the key parameter to describe wet deposition are similar to those expressed above for choosing $\mathrm{W}_{\mathrm{g}}$. For rain, $\mathrm{W}_{\mathrm{p}}$ can be investigated with relationships between particle size and washout ratios that have been developed from semiempirical derivations (Slinn, 1997; Slinn et al., 1978) and from field experiments (Gatz, 1975). Ratios for particle washout by snow have been predicted from theory to be smaller by approximately a factor of 10 (Scott, 1981). However, other theoretical evaluations and field measurements have suggested that below-cloud scavenging of particles by snow may be more efficient than scavenging by rain because of the larger size and surface area of snowflakes. McVeety and Hites (1988) found that measured particle washout ratios of PAHs for rain were greater by a factor of 2-4 than the ratios for snow, while Franz and Eisenreich (1998) found that snow was more efficient at removing particle-phase PAHs. Here $\mathrm{W}_{\mathrm{p}}$ is assumed to be the same for snow as for rain, because no easily generalized, universal method has been found from the various theoretical evaluations and field measurements.

The particle washout ratio can be investigated with the following semiempirical expression (Slinn, 1977):

$$
\mathrm{W}_{\mathrm{p}}=\mathrm{h}_{\mathrm{w}} \mathrm{E}_{\mathrm{o}} / \mathrm{D}_{\mathrm{m}}
$$

Here $h_{\mathrm{W}}$ is the effective height from which the chemical is removed by precipitation $(\sim 500 \mathrm{~m}$ in typical applications), $\mathrm{E}_{\mathrm{O}}$ is the collision efficiency of the particle and raindrop and is a function of particle size, and $\mathrm{D}_{\mathrm{m}}$ is the mean drop diameter (typically $1.0 \mathrm{~mm}$ ). Field measurements of various anthropogenically derived organic compounds indicate that they accumulate in the fine aerosol mode, although some studies have found high concentrations in the coarse fraction. The particle size distribution of the HAPs is related to the process by which they are emitted.

Combustion processes generate organics in the fine aerosol mode, and the organic HAPs that are emitted as vapors will preferentially sorb to fine particles, which contain the highest levels of organic matter. For a brief comparative analysis here of two approaches of estimating $\mathrm{W}_{\mathrm{p}}$, the HAPs can be assumed to be associated with particles with a mass median diameter $\left(\mathrm{D}_{\mathrm{mm}}\right)$ of $1 \mu \mathrm{m}$. The collision efficiency for a $1-\mu \mathrm{m}$-diameter particle is $4 \times 10^{-4}$ (Slinn, 1977; Slinn et al., 1978). The value of $W_{p}$ from equation (29) is then found to be $200 \mathrm{~m}^{3}$ air ( $\mathrm{m}^{3}$ rain)-1, a value that is smaller by a factor of 10-1000 than the measured values for PAHs and PCBs (Poster and Baker, 1996; Franz and Eisenreich, 1998). With the relationship for $\mathrm{W}_{\mathrm{p}}$ developed from field measurements (Gatz, 1975), the estimated value for a 1- $\mu$ m-diameter particle is approximately 
$1.4 \times 10^{5} \mathrm{~m}^{3}$ air $\left(\mathrm{m}^{3} \text { rain }\right)^{-1}$, which is a factor of 700 greater than the value estimated with equation (29) and on the high end of the measured values of washout ratios for PCBs and PAHs $\left(10^{3}-10^{5}\right)$. With the field-determined relationship between $\mathrm{W}_{\mathrm{p}}$ and $\mathrm{D}_{\mathrm{mm}}$, a value of $10^{4}$ would correspond to a particle with a $\mathrm{D}_{\mathrm{mm}}$ value of approximately $0.1 \mu \mathrm{m}$. For this analysis of the HAPs, a value of $\mathrm{W}_{\mathrm{p}}=10^{4} \mathrm{~m}^{3}$ air $\left(\mathrm{m}^{3} \text { rain) }\right)^{-1}$ (the midpoint of the range of measured values for PCBs and PAHs) is most appropriate, as a compromise for the semiempirical and fielddetermined relationships between washout ratios and particle size.

The washout ratio for inorganic particles is based on reasoning similar to that given above. Although the relationship between $\mathrm{W}_{\mathrm{p}}$ and $\mathrm{D}_{\mathrm{mm}}$ is not intrinsically linear, the approximation of linearity leads to estimates of $\mathrm{W}_{\mathrm{p}}$ that are well within uncertainties in the modeling and observational results shown by Gatz (1975), Slinn (1977), and similar studies on washout.

$$
\mathrm{W}_{\mathrm{p}}=10^{5} \mathrm{D}_{\mathrm{mm}}
$$

The values of $\mathrm{D}_{\mathrm{mm}}$ recommended for particulate HAPS, including polycyclic organic matter $(\mathrm{POM})$, are given in Appendix B and are based roughly on the approximation

$$
\mathrm{D}_{\mathrm{mm}}=1.5-1.5 x^{-1}
$$

where $\mathrm{D}_{\mathrm{mm}}$ has units of micrometers and $x$ is the fraction, if nonzero, of the mass in the fine mode.

Implementation of equation (28) with the existing ISC algorithms can be accomplished with procedures similar to those described in Section 4 for wet deposition of gases. Because particles are not released significantly from precipitation falling through areas of relatively low particle concentration, however, the formulas corresponding to equations (26) and (27) need to be modified when the plume does not extend to the surface. That is, the integral in equation (26) should be evaluated between the height $\mathrm{z}_{\mathrm{b}}$ of the bottom edge of the plume and height $\mathrm{z}_{\mathrm{t}}$, and the term $\mathrm{z}_{\mathrm{t}}{ }^{-1}$ outside the integral should be replaced by $\Delta \mathrm{z}^{-1}=\left(\mathrm{z}_{\mathrm{t}}-\mathrm{zb}_{\mathrm{b}}\right)^{-1}$. Also, in equation $(27), \mathrm{z}_{\mathrm{t}}^{-1}$ should be replaced by $\Delta \mathrm{z}^{-1}$.

Near emission sources where the vertical extent of the plume is small, relatively little time would be available for removal of particles from air as the precipitation falls through the plume. In this case, equations (29) and (30) would produce excessively large values of $F_{p}$. To address this difficulty, a minimum value of $\Delta z$ should be used in the formulas corresponding to equations (26) and (27). We assume that a minimum value of $500 \mathrm{~m}$ for $\Delta \mathrm{z}$ is appropriate and reasonably representative of mixed-layer heights during the field experiments cited above in the discussion leading to equation (30).

Washout can affect estimates of the dry deposition of particles. To evaluate the effect, the plume depletion factor for particle washout should be computed with the constraints noted above and then applied to adjust particle concentration values. The plume depletion factor need not be applied to particle dry deposition calculations when the plume does not extend downward to the height for which the dry deposition velocity is calculated. 


\section{Physicochemical Properties and Related Topics}

\subsection{Properties of Gases}

Values of the basic and relevant physicochemical properties of the gaseous HAPs are listed in Appendices $\mathrm{C}$ and D. For the properties that depend on temperature, a temperature of 298.2 $\mathrm{K}$ is assumed. The diffusivities $\mathrm{D}_{\mathrm{a}}$ shown in Appendix $\mathrm{C}$ for the substances in air were evaluated by using the following equation derived by Fuller et al. (1966, 1969):

$$
\mathrm{D}_{\mathrm{a}}=\left(0.143 \mathrm{~T}_{\mathrm{a}}{ }^{1.75}\right)\left\{\mathrm{PM}_{\mathrm{ax}}{ }^{0.5}\left[\left(\sum_{\mathrm{v}}\right)_{\mathrm{a}} 0.33+\left(\sum_{\mathrm{v}}\right)^{0.33}\right]^{2}\right\}^{-1},
$$

where

$$
\begin{aligned}
& \mathrm{T}_{\mathrm{a}}=\text { air temperature }(\mathrm{K}), \\
& \mathrm{P} \\
& \mathrm{M}_{\mathrm{ax}}=2\left[\left(1 / \mathrm{M}_{\mathrm{a}}\right)+(1 / \mathrm{M})\right]^{-1}, \\
& \mathrm{M}_{\mathrm{a}}=\text { molecular weight of air }\left(28.966 \mathrm{~g} \mathrm{~mol}^{-1}\right), \\
& \mathrm{M}=\text { molecular weight of compound, from Appendix } \mathrm{C}\left(\mathrm{g} \mathrm{mol}^{-1}\right), \\
& \left(\sum_{\mathrm{v}}\right)_{\mathrm{a}}=\text { molecular volume of air }(19.7), \\
& \sum_{\mathrm{v}}=\text { molecular volume of the compound. }
\end{aligned}
$$

The molecular volumes were estimated by summing the atomic diffusion volumes listed in Table 5 (Reid et al., 1987).

Values listed in Appendix $\mathrm{C}$ for the diffusivity $\mathrm{D}_{\mathrm{W}}$ of the compounds in water were estimated by using following correlation derived by Hayduk and Minhas (1982):

$$
\mathrm{D}_{\mathrm{W}}=1.25 \times 10^{-8}\left(\mathrm{~V}^{-0.19}-0.292\right) \mathrm{T}_{\mathrm{a}}^{1.52} \eta_{\omega} \mathrm{e}^{(9.58 / \mathrm{V}-1.12)},
$$

where $\quad \begin{array}{ll}\mathrm{V} & =\text { Le Bas molar volume }\left(\mathrm{cm}^{3} \mathrm{~mol}^{-1}\right), \\ \mathrm{T}_{\mathrm{a}} & =\text { air temperature }(\mathrm{K}), \\ \eta_{\omega} & \left.=\text { absolute viscosity of water (centipoise, } 10^{3} \mathrm{~Pa} \mathrm{~s}\right) .\end{array}$

The Le Bas molar volumes were estimated by summing the volume increments listed in Table 6 (Reid et al., 1987).

The recommended values of Henry's Law constants $(\mathrm{H})$ are listed in Appendix D. The Henry's Law constant is effectively an air-water partition coefficient and, by convention, is expressed as the ratio of the partial pressure (here expressed in units of Pascals) of the chemical in air to the concentration (here expressed in units of moles per cubic meter) of the chemical in water. Except where the aqueous solubility is noted as miscible, the values of $\mathrm{H}$ in Appendix D were estimated as the ratio of the chemical's vapor pressure to its aqueous solubility. The values of the vapor pressures and aqueous solubilities were obtained from compilations of the physicochemical properties of organic compounds by Mackay et al. (1992a and b, 1993, 1995). This method of calculating $\mathrm{H}$ is unreliable for high aqueous concentrations of the solute, that is, for mole fractions in excess of 0.01 or for aqueous concentrations of $1 \times 10^{4}$ mole $\mathrm{m}^{-3}$ (Mackay and Shiu, 1981). For chemicals with such miscibility in water, the values of $\mathrm{H}$ in Appendix D were derived from experimental results tabulated by two databases (Mackay et al., 1992a and b, 
1993, 1995; Sander, 1997). To illustrate the uncertainties in estimates of H, ranges of reported values from the two data bases are listed in Appendix E. A factor of two in the reported values is common.

Table 5. Atomic diffusion volumes for the calculation of molecular volumes $\sum_{v}$ (Reid et al., 1987).

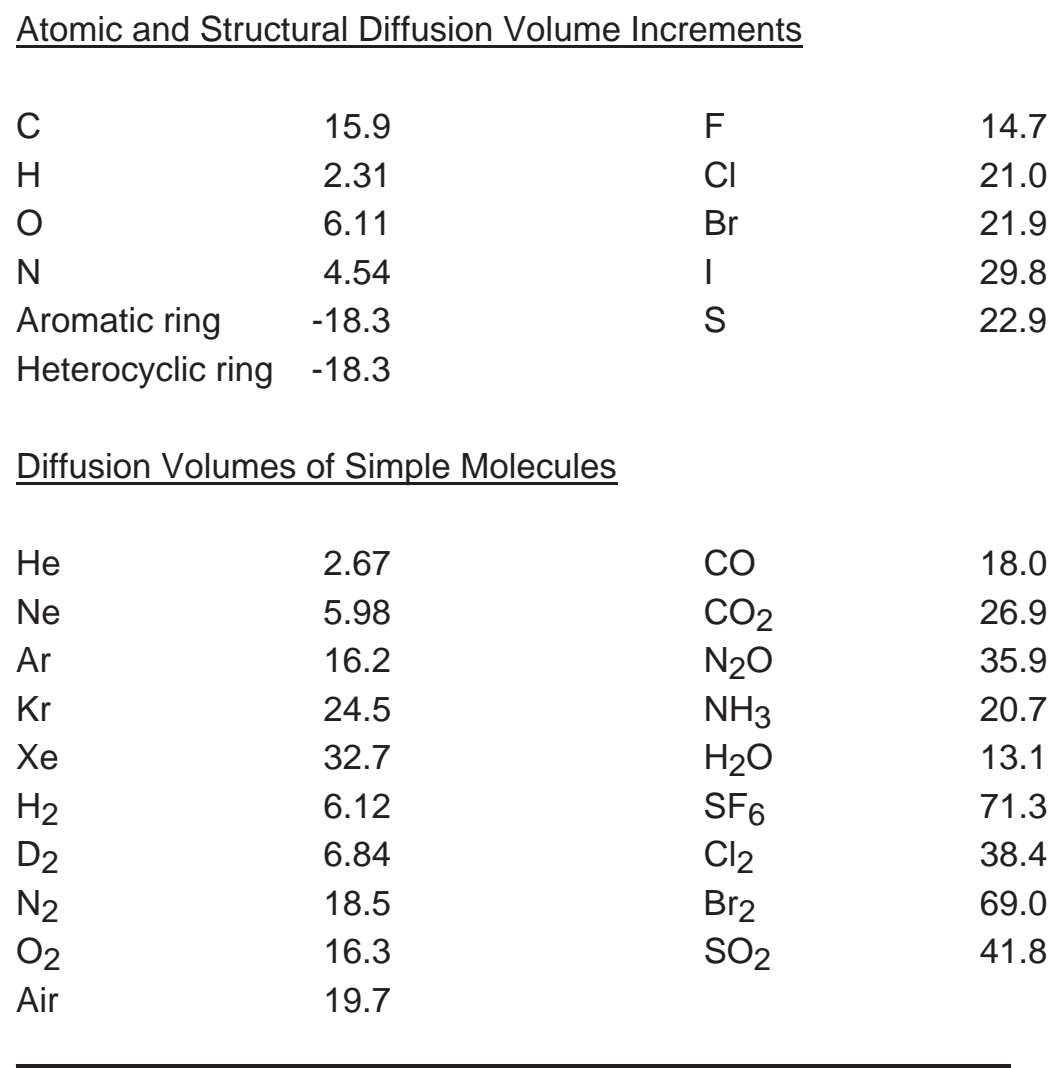

\subsection{Properties of Mixtures}

Several mixtures are included in the list of organic HAPs: PCBs, dibenzo-p-dioxins, dibenzofurans, POM, and coke oven emissions. Appendices C and D contain separate tables for each of these mixtures. To use these tables effectively, the components of the mixture must be specified by the ISC user. For example, PCBs are emitted as Aroclor mixtures, which are composed of PCB congeners having unique physicochemical properties.

All values of $\mathrm{H}$ for the PCB congeners in Appendix D were taken from Dunnivant et al. (1992). These researchers derived the values from structure-property relationships that utilized experimentally derived constants for a suite of PCB congeners (Dunnivant et al., 1988) to evaluate the relationship. 
In addition to the mixtures listed in Appendices $\mathrm{C}$ and $\mathrm{D}$, glycol ethers are listed as a HAPs. This mixture includes two substances: diethylene glycol monobutyl ether and ethylene glycol monobutyl ether. As indicated in Appendices C and D, insufficient information is available to evaluate the physicochemical properties of these two substances (numbers 58 and 82 in Appendices $\mathrm{C}$ and $\mathrm{D})$.

Table 6. Volume increments for the calculation of the

Le Bas molar volumes V (Reid et al., 1987).

\begin{tabular}{lr}
\hline Compound & $\begin{array}{r}\text { Increment } \\
\left(\mathrm{cm}^{3} \mathrm{~mol}^{-1}\right)\end{array}$ \\
\hline & \\
\hline Carbon & 14.8 \\
Hydrogen & 3.7 \\
Oxygen (except as noted below) & 7.4 \\
$\quad$ In methyl esters and ethers & 9.1 \\
$\quad$ In ethyl esters and ethers & 9.9 \\
$\quad$ In higher esters and ethers & 11.0 \\
$\quad$ In acids & 12.0 \\
Joined to S, P, or N & 8.3 \\
Nitrogen & \\
$\quad$ Doubly bonded & 15.6 \\
$\quad$ In primary amines & 10.5 \\
In secondary amines & 12.0 \\
Bromine & 27 \\
Chlorine & 24.6 \\
Fluorine & 8.7 \\
lodine & 37 \\
Sulfur & 25.6 \\
Ring, three membered & -6.0 \\
Four membered & -8.5 \\
Five membered & -11.5 \\
Nix membered & -15.0 \\
Anthracene & -30.0 \\
Double bond between carbon atoms & -47.5 \\
Triple bond between carbon atoms & - \\
& - \\
\hline & \\
& \\
&
\end{tabular}




\subsection{Particle-Gas Partitioning}

The rate at which a chemical substance is deposited by dry and wet deposition depends on the partitioning of the substance between the gaseous and particulate forms. The fraction $\phi$ of the chemical sorbed on particles can be expressed in terms of the ambient total suspended particulate matter (TSP):

$$
\phi=\mathrm{K}_{\mathrm{p}}(\mathrm{TSP}) /\left[1+\mathrm{K}_{\mathrm{p}}(\mathrm{TSP})\right],
$$

where

$$
\begin{aligned}
\mathrm{K}_{\mathrm{p}} & =\text { particle-gas partition coefficient }\left(\mathrm{m}^{3} \mu \mathrm{g}^{-1}\right), \\
\mathrm{TSP} & =\text { ambient total suspended particulate matter }\left(\mu \mathrm{g} \mathrm{m}^{-3}\right) .
\end{aligned}
$$

Thus, given the concentration of a HAP in both gaseous and particulate form, the fraction $\phi$ is treated as particles in application of the dry and wet deposition formulations, and the fraction (1 $\phi)$ is treated as a gas. One difficulty with this approach is that local measurements of TSP are not always available, and the actual values can vary substantially with time and from location to location. Typical values for the amount of material in particles smaller than $10 \mu \mathrm{m}$ in diameter $\left(\mathrm{PM}_{10}\right.$, a component of TSP) in urban areas have been reported to be near $100 \mu \mathrm{g} \mathrm{m}^{-3}$ (Seinfeld and Pandis, 1998). In the United States, however, the amount of coarse particulate material in urban areas now tends to smaller, in part because of efforts to meet the national air quality standard of $50 \mu \mathrm{g} \mathrm{m}^{-3}$ for the annual average $\mathrm{PM}_{10}$. Here, a value of TSP $=50 \mu \mathrm{g} \mathrm{m}^{-3}$ is recommended if no other information is available on TSP or $\mathrm{PM}_{10}$ amounts at a specific location.

To apply equation (34), $\mathrm{K}_{\mathrm{p}}$ must be evaluated. Three models have been developed to estimate the sorption of semivolatile organic compounds to particles: the Junge-Pankow Adsorption model (Pankow, 1987), the Mackay Adsorption model (Mackay et al., 1986), and the Octanol-Air Partition Coefficient model (Finizio et al., 1997). An assessment by Bidleman and Harner (2000) of the predictive capability of these models for several organochlorine compounds showed that the agreement varied with the chemical compound but that, in general, the OctanolAir Partition Coefficient model gave somewhat lower results and the Mackay Adsorption model somewhat higher results than the Junge-Pankow Adsorption model. The Octanol-Air Partition Coefficient model was chosen here to estimate the gas-particle partitioning of the HAPs. To apply this approach and provide the values of $\log \mathrm{K}_{\mathrm{p}}$ listed in Appendix D, the following equation was applied:

$$
\log \mathrm{K}_{\mathrm{p}}=\log \mathrm{K}_{\mathrm{oa}}-12.61 .
$$

Here $\mathrm{K}_{\mathrm{oa}}=\mathrm{K}_{\mathrm{ow}} \mathrm{RT}_{\mathrm{a}} / \mathrm{H}$ is the octanol-air partition coefficient, where $\mathrm{K}_{\mathrm{ow}}$ is the octanol-water partition coefficient.

\subsection{Evaluation of $r_{\mathrm{cl}}$}

Appendix D lists the values of $r_{c l}$ that are necessary for application of equation (12). This term represents the resistance to uptake by individual leaves in a vegetative canopy and was 
evaluated on the basis of theory and data from laboratory experiments. This resistance was considered in terms of cuticle permeability, which depends on the concentration of a substance in the cuticle and its mobility (Kerler and Schönherr, 1988a,b; Riederer, 1990; Paterson et al., 1991). The concentration of the chemical in the cuticle was estimated from partition coefficients, and its mobility was derived from diffusion coefficients. To estimate $r_{c l}$ for semivolatile organic compounds and other chemicals with saturation vapor pressures $\mathrm{p}^{\circ}$ less than $125 \mathrm{~Pa}$, the following correlations developed by Kerler and Schönherr (1988a,b) were used:

$$
\begin{aligned}
& \log \mathrm{K}_{\mathrm{cw}}=0.973 \log \mathrm{K}_{\mathrm{ow}}+0.045, \\
& \log \mathrm{P}_{\mathrm{cw}}=\left(238 \log \mathrm{K}_{\mathrm{cw}}\right) \mathrm{V}_{\mathrm{m}}{ }^{-1}-12.48,
\end{aligned}
$$

where $\quad \mathrm{K}_{\mathrm{cw}}=$ cuticle-water partition coefficient (unitless),

$\mathrm{K}_{\mathrm{ow}}=1$-octanol-water partition coefficient (unitless),

$\mathrm{P}_{\mathrm{cW}}=$ permeance coefficient $\left(\mathrm{m} \mathrm{s}^{-1}\right)$,

$\mathrm{V}_{\mathrm{m}} \quad=$ molar volume of the liquid chemical at the normal boiling point $\left(\mathrm{cm}^{3} \mathrm{~mol}^{-1}\right)$.

Here $\mathrm{K}_{\mathrm{OW}}$ is the coefficient described by Leo et al. (1971) and Chiou et al. (1982), and $\mathrm{V}_{\mathrm{m}}$ was derived by the Le Bas estimation method (Reid et al., 1987). The term $\mathrm{P}_{\mathrm{cw}}$ represents the cuticle conductance derived for the aqueous phase and has units of velocity. The corresponding term $\mathrm{P}_{\mathrm{ca}}$ for the gaseous phase was derived by multiplying $\mathrm{P}_{\mathrm{cw}}$ by RT/H. Kerler and Schönherr (1988b) developed the correlations for $\mathrm{P}_{\mathrm{cw}}$ by using cuticle membranes that were $2.5 \mu \mathrm{m}$ thick. Values of $\mathrm{P}_{\mathrm{ca}}$ were adjusted to a cuticular membrane thickness of $1.0 \mu \mathrm{m}$, which is more representative of plant leaves, by multiplying $\mathrm{P}_{\mathrm{ca}}$ by $(2.5 / 1.0)^{2}$. Then $\mathrm{r}_{\mathrm{cl}}$ was found as the inverse of $\mathrm{P}_{\mathrm{ca}}$.

For volatile organic compounds $\left(\mathrm{p}^{\circ}>125 \mathrm{~Pa}\right)$, the cuticle-water partition coefficient was estimated with the method developed by Welke et al. (1998):

$$
\begin{aligned}
& \log \mathrm{K}_{\mathrm{ca}}=6176-0.892 \log \mathrm{p}^{\circ}, \\
& \mathrm{K}_{\mathrm{cw}}=\mathrm{K}_{\mathrm{ca}} \mathrm{H}\left(\mathrm{RT}_{\mathrm{a}}\right)^{-1} .
\end{aligned}
$$

Then equation (34) was applied, and $\mathrm{r}_{\mathrm{cl}}$ was found as already described. The intermediate variables of $\log \mathrm{K}_{\mathrm{Ow}}$ and $\log \mathrm{K}_{\mathrm{cw}}$ are listed in Appendix D. With the exception of the $\log \mathrm{K}_{\mathrm{Ow}}$ values for the PCB congeners, all values for the individual HAPs and HAPs mixtures in Appendix D were those recommended by Mackay et al. (1992a and b, 1993, 1995). Values for the PCB congeners were derived from quantitative structure-property relationships by Hansen et al. (1999). Ranges of $\log \mathrm{K}_{\mathrm{ow}}$ values listed in Appendix $\mathrm{E}$ were collected from the database of Mackay et al. (1992a and b, 1993, 1995), which contains an extensive compilation of constants determined by experiment, calculation, and quantitative structure-property relationships. 


\subsection{Inorganic Gases}

The inorganic gases $\mathrm{HCl}, \mathrm{HF}, \mathrm{TiCl}_{4}$, and the nonparticulate forms of $\mathrm{Hg}$ are HAPs that require special consideration in this report. These substances are inorganic gases that do not fit well into the categories of substances addressed in Appendices B-E. The properties of these substances can be found in standard references such as the CRC Handbook of Chemistry and Physics and The Merck Index-Enclylopedia of Chemicals, Drugs, and Biologicals. Table 7 summarizes approximate, nominal, or prescribed values for the physiochemical parameters derived from the suggestions in this section.

The compounds $\mathrm{HCl}$ and $\mathrm{HF}$ are extremely soluble in water. As a result, the two substances have negligibly small values of $\mathrm{R}_{\mathrm{c}}$ for dry deposition and very large washout ratios $\mathrm{W}_{\mathrm{g}}$ for wet deposition. A procedure sufficient to produce the values desired for $\mathrm{R}_{\mathrm{c}}$ and $\mathrm{W}_{\mathrm{g}}$ with the gaseous deposition algorithms assumes that the values of $\mathrm{H}$ are very small, $10^{-12} \mathrm{~Pa} \mathrm{~m}^{3} \mathrm{~mol}^{-1}$ or less. The other key variables needed in the dry deposition algorithms can be set to nominal or prescribed values, specifically $\mathrm{D}_{\mathrm{a}}=0.3 \times 10^{-4} \mathrm{~m}^{2} \mathrm{~s}^{-1}, f_{\mathrm{o}}=0$, and $\mathrm{r}_{\mathrm{cl}}=10^{7} \mathrm{~s} \mathrm{~m}^{-1}$.

Table 7. Suggested values of key physicochemical properties of inorganic gases.

\begin{tabular}{lllll}
\hline Substance & \multicolumn{1}{c}{$\begin{array}{c}\mathrm{H} \\
\left(\mathrm{Pa} \mathrm{m}^{3} \mathrm{~mol}^{-1}\right)\end{array}$} & $f_{\mathrm{O}}$ & $\begin{array}{c}\mathrm{D}_{\mathrm{a}} \\
\left(\mathrm{m}^{2} \mathrm{~s}^{-1}\right)\end{array}$ & $\begin{array}{c}\mathrm{r}_{\mathrm{Cl}} \\
\left(\mathrm{s} \mathrm{m}^{-1}\right)\end{array}$ \\
\hline $\mathrm{HCl}$ & $10^{-12}$ & 0 & $0.3 \times 10^{-4}$ & $10^{7}$ \\
$\mathrm{HF}$ & $10^{-12}$ & 0 & $0.3 \times 10^{-4}$ & $10^{7}$ \\
$\mathrm{TiCl}_{4}$ & $10^{-12}$ & 1 & $0.08 \times 10^{-4}$ & $10^{7}$ \\
$\mathrm{Hg}^{0}$ & 150 & 0 & $0.07 \times 10^{-4}$ & $10^{7}$ \\
$\mathrm{Hg}^{2+}$ & $6 \times 10^{-6}$ & 1 & $0.06 \times 10^{-4}$ & $10^{7}$ \\
& & & & \\
\hline
\end{tabular}

The gas $\mathrm{TiCl}_{4}$ reacts rapidly with moisture to produce $\mathrm{TiO}_{2}$ and $\mathrm{HCl}$ and is sufficiently soluble in water to allow the reaction to proceed quickly. The desired results can be achieved with the deposition algorithms by setting the value of $\mathrm{H}$ to $10^{-12} \mathrm{~Pa} \mathrm{~m}^{3} \mathrm{~mol}^{-1}$ or smaller and the value of $f_{\mathrm{o}}$ to 1 . Other variables needed in the dry deposition algorithms can be set to nominal or prescribed values, specifically $\mathrm{D}_{\mathrm{a}}=0.08 \times 10^{-4} \mathrm{~m}^{2} \mathrm{~s}^{-1}$ and $\mathrm{r}_{\mathrm{cl}}=10^{7} \mathrm{~s} \mathrm{~m}^{-1}$.

The deposition velocities for $\mathrm{HCl}, \mathrm{HF}$, and $\mathrm{TiCl}_{4}$ are expected to be similar to those for $\mathrm{HNO}_{3}$, which have been observed and modeled to be quite large, 1-4 cm s${ }^{-1}$ over many surfaces. To avoid the possibility of computational difficulties involving these gases, the practice used by Wesely (1989) for $\mathrm{HNO}_{3}$ of setting a lower limit of $10 \mathrm{~s} \mathrm{~m}^{-1}$ for $\mathrm{R}_{\mathrm{c}}$ should be followed for all substances. 
Titanium can also occur in particulate form in the atmosphere, for example as $\mathrm{TiO}_{2}$ generated after exposure of resuspendable materials to $\mathrm{TiCl}_{4}$. For a scenario in which soils and plant materials are exposed to high levels of $\mathrm{TiCl}_{4}$, dust particles containing $\mathrm{TiO}_{2}$ could conceivably become airborne. In this case, the particulate material would probably all be in the coarse portion of the particle size distribution, and a value of $\mathrm{D}_{\mathrm{mm}}=7 \mu \mathrm{m}$ can be assumed, commensurate with values shown in Appendix B.

Two gaseous forms of $\mathrm{Hg}$ are common: elemental and divalent mercury. Only the particulate forms of $\mathrm{Hg}$ are addressed in Appendix B. The elemental form $\left(\mathrm{Hg}^{0}\right)$ is emitted in the vapor phase, but its low solubility in water and its chemical stability cause its deposition velocity to be small. When the concentration of $\mathrm{Hg}^{0}$ in the air is small, the element can be emitted from the surface (e.g., Hanson et al., 1995). Thus, $\mathrm{Hg}^{0}$ can be recycled between the atmosphere and the surface, contributing to a longevity as great as one year in the atmosphere. Here we neglect emission from natural surfaces. For deposition, the key physicochemical parameter is $\mathrm{H}$, for which a value of $150 \mathrm{~Pa} \mathrm{~m}^{3} \mathrm{~mol}^{-1}$ is appropriate (e.g., Pirrone et al., 1995a). This value of $\mathrm{H}$ can be used with nominal values of $D_{a}=0.07 \times 10^{-4} \mathrm{~m}^{2} \mathrm{~s}^{-1}, f_{\mathrm{o}}=0$, and $\mathrm{r}_{\mathrm{cl}}=10^{7} \mathrm{~s} \mathrm{~m}^{-1}$ in the deposition algorithms to produce realistic values of $R_{c}$ and $W_{g}$. The resulting estimates of $V_{d g}$ over nonvegetated surfaces and of $F_{g}$ are very small. The estimates of $V_{d g}$ above vegetation peak at values less than $0.1 \mathrm{~cm} \mathrm{~s}^{-1}$ during the daytime and are negligible at night (e.g., see modeling results of Shannon and Voldner, 1995).

The divalent form $\mathrm{Hg}^{2+}$ is often a small fraction of the gaseous $\mathrm{Hg}$ emissions from anthropogenic sources but can account for a fairly large fraction of the $\mathrm{Hg}$ deposited at the surface near the sources (e.g., Lindberg and Stratton, 1998). Here we ignore emission and deposition of biologically mediated forms of $\mathrm{Hg}$ such as methylmercury. The value of $\mathrm{H}$ for $\mathrm{HgCl}_{2}$, perhaps the most common form of $\mathrm{Hg}^{2+}$ emitted from anthropogenic sources, is near $5 \times 10^{-5} \mathrm{~m}^{3} \mathrm{~mol}^{-1}$ in acidic solutions and tends to be smaller as acidity decreases (Sommar et al., 2000). A value of $\mathrm{H}$ more appropriate for ISC deposition calculations can be estimated as the vapor pressure divided by the aqueous solubility in ambient environmental conditions. The vapor pressure at $25^{\circ} \mathrm{C}$ is assumed to be $0.15 \mathrm{~Pa}$, roughly midway between reported values of $0.27 \mathrm{~Pa}$ for elemental $\mathrm{Hg}$ and $0.033 \mathrm{~Pa}$ for $\mathrm{HgI}_{2}$, and the aqueous solubility of $\mathrm{HgCl}_{2}$ is approximately

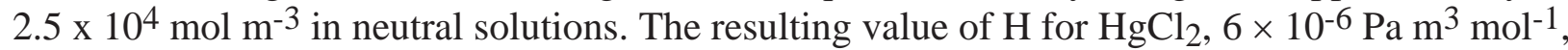
indicates that the coefficient $\mathrm{W}_{\mathrm{g}}$ and the potential washout rates for $\mathrm{Hg}^{2+}$ are large. Deposition velocities computed with this estimate of $H$, together with the values $D_{a}=0.06 \times 10^{-4} \mathrm{~m}^{2} \mathrm{~s}^{-1}, \mathrm{r}_{\mathrm{cl}}$ $=10^{7} \mathrm{~s} \mathrm{~m}^{-1}$, and $f_{\mathrm{o}}=1$ (the latter to indicate that $\mathrm{HgCl}_{2}$ is a strong oxidizing agent in water), support the contention that this form of $\mathrm{Hg}$ can be taken up rapidly by natural surfaces. The values of $\mathrm{V}_{\mathrm{dg}}$ computed will peak at several centimeters per second, and vertical transport by dry deposition will be limited primarily by gas-phase resistances $\mathrm{R}_{\mathrm{a}}$ and $\mathrm{R}_{\mathrm{b}}$. Such behavior has been simulated (e.g., Shannon and Voldner, 1995) and is commensurate with limited observations above forest (Lindberg and Stratton, 1998). 


\subsection{Atmospheric Half-Life}

The overall atmospheric half-lives $\mathrm{t}_{1 / 2}$ ( $\mathrm{s}$ ) of the gas-phase HAPs are an important consideration for short-range dispersion of highly reactive substances. Here, a method is outlined to estimate $t_{1 / 2}$. Examples of some calculated values and some reported by Spicer et al. (1993) are shown in Appendix F. The half-life can be estimated from the rate constants of the reactions of the HAPs with the major oxidants in the atmosphere: hydroxyl radical $(\mathrm{OH})$, nitrate radical $\left(\mathrm{NO}_{3}\right)$, and $\mathrm{O}_{3}$. Photolysis should also be considered for some of the HAPs. The overall atmospheric half-life is calculated as

$$
\mathrm{t}_{1 / 2}=0.693\left\{\mathrm{kOH}[\mathrm{OH}]+\mathrm{kNO}_{3}\left[\mathrm{NO}_{3}\right]+\mathrm{kO}_{3}\left[\mathrm{O}_{3}\right]+\mathrm{k}_{\mathrm{phot}}\right\}^{-1},
$$

where

$$
\begin{aligned}
& \mathrm{k}_{\mathrm{OH}}=\text { reaction rate of the } \mathrm{HAP} \text { with } \mathrm{OH}\left(\mathrm{cm}^{3} \text { molecule }-1 \mathrm{~s}^{-1}\right) \\
& \mathrm{kNO}_{3}=\text { reaction rate of the } \mathrm{HAP} \text { with } \mathrm{NO}_{3}\left(\mathrm{~cm}^{3} \text { molecule } \mathrm{e}^{-1} \mathrm{~s}^{-1}\right) \\
& \mathrm{k}_{3}=\text { reaction rate of the } \mathrm{HAP} \text { with } \mathrm{O}_{3}\left(\mathrm{~cm}^{3} \text { molecule-1 } \mathrm{s}^{-1}\right), \\
& \mathrm{k}_{\mathrm{phot}}=\text { photolysis rate constant of the } \mathrm{HAP}\left(\mathrm{s}^{-1}\right), \\
& {[\mathrm{OH}]=\text { concentration of } \mathrm{OH}\left(\text { molecule } \mathrm{cm}^{-3}\right),} \\
& {\left[\mathrm{NO}_{3}\right]=\text { concentration of } \mathrm{NO}\left(\text { molecule } \mathrm{cm}^{-3}\right),} \\
& {\left[\mathrm{O}_{3}\right]=\text { concentration of } \mathrm{O}_{3}\left(\text { molecule } \mathrm{cm}^{-3}\right) .}
\end{aligned}
$$

The values of the calculated half-lives listed in Appendix $\mathrm{F}$ were found with the assumption that the concentrations were $[\mathrm{OH}]=1 \times 10^{6}$ molecule $\mathrm{cm}^{-3}$ and $\left[\mathrm{O}_{3}\right]=1.5 \times 10^{12}$ molecule cm${ }^{-3}$ (60 ppbv). Values of the rate constants $\mathrm{k}_{\mathrm{OH}}, \mathrm{kNO}_{3}$, and $\mathrm{k}_{3}$, were obtained reaction rate data compiled by Atkinson (1994). Reaction with $\mathrm{NO}_{3}$ can be important at night and is quite rapid for many of the HAPs, but this pathway was neglected because of insufficient data on concentrations of $\mathrm{NO}_{3}$ in the nighttime atmosphere. The photolysis rates also were not evaluated. 


\section{References}

Abramopoulos, F., C. Rosenzweig, and B. Choudhury, 1988: Improved ground hydrology calculations for global climate models (GCMs): Soil water movement and evapotranspiration. J. Clim., 1, 921-941.

Ames, M., G. Gullu, and I. Olmez, 1998: Atmospheric mercury in the vapor phase, and in fine and coarse particulate matter at Perch River, New York. Atmos. Environ., 32, 865-872.

Atkinson, R., 1994: Gas-phase tropospheric chemistry of organic compounds. J. Phys. Chem. Ref. Data, Monograph 2, American Chemical Society, Washington, D.C.

Atkinson, D. G., D. T. Bailey, J. S. Irwin, and J. S. Touma, 1997: Improvements to the EPA industrial source complex dispersion model. J. Appl. Meteorol., 36, 1088-1095.

Baldocchi, D. D., 1993: Deposition of gaseous sulfur compounds to vegetation. In Sulfur Nutrition and Assimilation and Higher Plants, edited by L. J. Kok et al., pp. 271-293, SGP Academic, The Hauge, Netherlands.

Bamford, H. A., D. L. Poster, and J. E. Baker, 2000: Henry's Law constants of polychlorinated biphenyl congeners and their variation with temperature. J. Chem. Eng. Data, 45, 1069-1074.

Betts, A., F. Chen, K. Mitchell, and Z. Janic, 1997: Assessment of the land surface and boundary layer models in two operational versions of the NCEP Eta model using FIFE data. Mon. Wea. Rev., 125, 2896-2915.

Bidleman, T. F., and T. Harner, 2000: Sorption to aerosols. In Handbook of Property Estimation Methods for Chemicals, edited by R. S. Boethling and D. Mackay, Lewis Publishers, Boca Raton.

Binkowski, F. S., and U. Shankar, 1995: The Regional Particulate Matter Model, 1. Model description and preliminary results. J. Geophys. Res., 100, 26,191-26,209.

Brunner, S., E. Hornung, H. Santl, E. Wolff, O. G. Piringer, J. Altschuh, and R. Brüggemann, 1990: Henry's Law constants for polychlorinated biphenyls: Experimental determination and structure-property relationships. Environ. Sci. Technol., 24, 1751-1754.

Brutsaert, W., 1982: Evaporation into the Atmosphere. D. Reidel Publishing Company, Dordrecht.

Buckman, H. O., and N. C. Brady, 1960: The Nature and Properties of Soils. The MacMillan Company, New York.

Byun, D. W., 1990: On the analytical solutions of flux-profile relationships for the atmospheric surface layer. J. Appl. Meteorol., 29, 652-657. 
Byun, D. W., and R. Dennis, 1995: Design artifacts in Eurlerian air quality models: Evaluation of the effects of layer thickness and vertical profile correction on surface ozone concentrations. Atmos. Environ., 29, 105-126.

Byun, D. W., J. E. Pleim, R. T. Tang, and A. Bourgeois, 1999: Meteorology-chemistry interface processor (MCIP) for Models-3 Community Multiscale Air Quality (CMAQ) modeling system. Ch. 12 in Science Algorithms for the EPA Models-3 Community Multiscale Air Quality (CMAQ) Modeling System, edited by D. W. Byun and J. K. S. Ching, pp. 12.1-12.78, EPA/600/R-99/030, U. S. Environmental Protection Agency, Office of Research and Development, Washington, D.C.

Cape J. N., L. J. Sheppard, J. Binnie, and A. L. Dickinson, 1998: Enhancement of the dry deposition of sulphur dioxide to a forest in the presence of ammonia. Atmos. Environ., 32, 519-524.

Caruso, E., G. M. Braga Marcazzan, and P. Redealli, 1981: PIXE investigation of element concentration and particle size distribution in Milan atmospheric aerosol. Nuc. Instr. Methods 181, 425-429.

Chiou, C. T., D. W. Schmedding, and M. Manes, 1982: Partitioning of organic compounds in octanol-water systems. Environ. Sci. Technol., 16, 4-10.

Deinum, G., A. C. Baart, D. J. Bakker, J. H. Duyzer, and K. D. van den Hout, 1995: The influence of uptake by leaves on atmospheric deposition of vapor-phase organics. Atmos. Environ., 29, 997-1005.

Delumyea, R., and R. L. Petel, 1979: Deposition velocity of phosphorus-containing particles over southern Lake Huron, April-October, 1975. Atmos. Environ., 13, 287-294.

Doran, J. C., and T. W. Horst, 1985: An evaluation of Gaussian plume-depletion models with dual-tracer field measurements. Atmos. Environ., 19, 939-951.

Dunnivant, F. M., J. T. Coates, and A. W. Elzerman, 1988: Experimentally determined Henry's Law constants for 17 polychlorobiphenyl congeners. Environ. Sci. Technol., 22, 448-453.

Dunnivant, F. M., A. W. Elzerman, P. C. Jurs, and M. N. Hasan, 1992: Quantitative structureproperty relationships for aqueous solubilities and Henry's law constants of polychlorinated biphenyls. Environ. Sci. Technol., 26, 1567-1573.

Enders G., R. Dlugi, R. Steinbrecher, B. Clement, R. Daiber, J. v. Eijk, S. Gäb, M. Haziza, G. Helas, U. Herrmann, M. Kessel, J. Kesselmeier, D. Kotzias, K. Kourtidis, H.-H. Kurth, R. T. McMillen, G. Roider, W. Schürmann, U. Teichmann, and L. Torres, 1992:

Biosphere/atmosphere interactions: Integrated research in a European coniferous forest ecosystem. Atmos. Environ., 26A, 171-189. 
Environmental Protection Agency, 1994: Modeling Fugitive Dust Impacts from Surface Coal Mining Operations-Phase II. EPA-454/R-94-025, U.S. Environmental Protection Agency, Office of Air Quality Planning and Standards, Research Triangle Park.

Environmental Protection Agency, 1995a: User's Guide for the Industrial Source Complex (ISC3) Dispersion Models. Volume I. User Instructions. EPA-454/B-95-003a, U.S. Environmental Protection Agency, Research Triangle Park.

Environmental Protection Agency, 1995b: User's Guide for the Industrial Source Complex (ISC3) Dispersion Models. Volume II. Description of Model Algorithms. EPA-454/B-95003b, U.S. Environmental Protection Agency, Research Triangle Park.

Erickson, M. D., 1997: Analytical Chemistry of PCBs. Lewis Publishers, Boca Raton.

Erisman, J. W., 1994: Evaluation of a surface resistance parameterization of sulphur dioxide. Atmos. Environ., 28, 2583-2594.

Erisman J. W., and G. P. Wyers, 1993: Continuous measurements of surface exchange of $\mathrm{SO}_{2}$ and $\mathrm{NH}_{3}$ : Implications for the possible interaction in the deposition process. Atmos. Environ., 27A, 1937-1949.

Finizio, A., D. Mackay, T. F. Bidleman, and T. Harner, 1997: Octanol-air partition coefficient as a predictor of partitioning of semi-volatile organic chemicals to aerosols. Atmos. Environ., 31, 2289-2296.

Fowler, D., 1978: Dry deposition of $\mathrm{SO}_{2}$ on agricultural crops. Atmos. Environ., 12, 369-373.

Franz, T. P., and S. J. Eisenreich, 1998: Snow scavenging of polychlorinated biphenyls and polycyclic aromatic hydrocarbons in Minnesota. Environ. Sci. Technol., 32, 1771-1778.

Fuentes, J. D., T. J. Gillespie, G. den Hartog, and H. H. Newman, 1992: Ozone deposition onto a deciduous forest during wet and dry conditions. Agric. For. Meteorol., 62, 1-18.

Fuller, E. N., P. D. Schettler, and J. C. Giddings, 1966: A new method for the prediction of gasphase diffusion coefficients. Ind. Eng. Chem., 58, no. 5, 19-27.

Fuller, E. N., K. Ensley, and J. C. Giddings, 1969: Diffusion of halogenated hydrocarbons in helium. The effect of structure on collision cross sections. J. Phys. Chem., 73, 3679-3685.

Gatz, D. F., 1975: Pollutant aerosol deposition into southern Lake Michigan. Water, Air, Soil Pollut., 5, 239-251.

Grantz, D. A., X. J. Zhang, W. J. Massman, G. den Hartog, H. H. Neumann, and J. R. Pederson, 1995: Effects of stomatal conductance and surface wetness on ozone deposition in fieldgrown grape. Atmos. Environ., 29, 3189-3198. 
Grimmond, C. S. B., T. S. King, M. Roth, and T. R. Oke, 1998: Aerodynamic roughness of urban areas derived from wind observations. Boundary-Layer Meteorol., 89, 1-24.

Hall, B., C. Claiborn, and D. Baldocchi, 1999: Measurement and modeling of the dry deposition of peroxides. Atmos. Environ., 33, 577-589.

Hansen, B. G., A. B. Paya-Perez, M. Rahman, and B. R. Larsen, 1999: QSARs for Kow and Koc of PCB congeners: A critical examination of data, assumptions and statistical approaches. Chemosphere, 39, 2209-2228.

Hanson, P. J., S. E. Lindberg, T. A. Tabberer, J. G. Owens, and K.-H. Kim, 1995: Foliar exchange of mercury vapor: Evidence for a compensation point. Water, Air, Soil Pollut., 80, 373-382.

Harrison, P. R., W. R. Matson, and J. W. Winchester, 1971: Time variations of lead, cooper and cadmium concentrations in aerosols in Ann Arbor, Michigan. Atmos. Environ. 5, 613-619.

Hayduk, W., and B. S. Minhas, 1982: Correlations for prediction of molecular diffusivities in liquids. Can. J. Chem. Eng., 60, 295-299.

Hicks, B. B., 1986: Differences in wet and dry particle deposition parameters between North America and Europe. In Aerosols: Research, Risk Assessment and Control Strategies, edited by S. D. Lee, T. Schneider, L. D. Grant, and P. J. Verkerk, pp. 973-982, Lewis Publishers, Inc., Chelsea, Michigan.

Hillel, D., 1998: Environmental Soil Physics. Academic Press, New York.

Hoff, J. T., F. Wania, D. Mackay, and R. Gillham, 1995: Sorption of nonpolar organic vapors by ice and snow. Environ. Sci. Technol., 29, 1982-1989.

Jannssen, L. H. J. M., and Römer, F. G., 1991: The frequency and duration of dew occurrence over a year. Tellus, 43B, 408-419.

Kaupp, H., and M. S. McLachlan, 1999: Atmospheric particle size distributions of polychlorinated dibenzo-p-dioxins and dibenzofurans (PCDD/Fs) and polycyclic aromatic hydrocarbons (PAHs) and their implications for wet and dry deposition. Atmos. Environ., 33, 85-95.

Keeler, G. J., W. W. Brachaczek, R. A. Gorse, Jr., S. M. Japar and W. R. Pierson, 1988: Effect of ambient humidity on dichotomous sampler coarse/fine ratios. Atmos. Environ., 22, 17151720.

Keeler, G., G. Glinsorn, and N. Pirrone, 1995: Particulate mercury in the atmosphere: Its significance, transport, transformation and sources. Water, Air \& Soil Pollut., 80, 159-168. 
Kerler, F., and J. Schönherr, 1988a: Accumulation of lipophilic chemicals in plant cuticles: Prediction from octanol/water partition coefficients. Arch. Environ. Contam. Toxicol., 17, 1-6.

Kerler, F., and J. Schönherr, 1988b: Permeation of lipophilic chemicals across plant cuticles: Prediction from partition coefficients and molar volumes. Arch. Environ. Contam. Toxicol., 17, 7-12.

Kim, J., and S. B. Verma, 1991: Modeling canopy stomatal conductance in a temperature grassland ecosystem. Agri. For. Meteorol., 55, 149-266.

Lee, R. E., Jr., and R. K. Patterson, 1969: Size determination of atmospheric phosphate, nitrate, chloride, and ammonium particulate in several urban areas. Atmos. Environ., 3, 249-255.

Lee, R. E., Jr., R. K. Patterson, and J. Wagman, 1968: Particle-size distribution of metal components in urban air. Environ. Sci. Technol., 2, 288-290.

Lee, R. E., Jr., S. S. Goranson, R. E. Enrione and G. G. Morgan, 1972: National air surveillance cascade impactor network. II: Size distribution measurements of trace metal components. Environ. Sci. Technol. 6, 1025-1030.

Leo, A., C. Hansch, and D. Elkins, 1971: Partition coefficients and their uses. Chem. Rev., 71, 525-616.

Lindberg, S. E., and W. J. Stratton, 1998: Atmospheric mercury speciation: Concentrations and behavior of reactive gaseous mercury in ambient air. Environ. Sci. Technol., 32, 49-57.

Mackay, D., S. Paterson, and W. H. Schroeder, 1986: Model describing the rates of transfer processes of organic chemicals between atmosphere and water. Environ. Sci. Technol., 20, 810-816.

Mackay, D., W. Y. Shiu, and K. C. Ma, 1992a: Illustrated Handbook of Physical-Chemical Properties and Environmental Fate for Organic Chemicals. Volume I. Monoaromatic Hydrocarbons, Chlorobenzenes, and PCBs. Lewis Publishers, Boca Raton.

Mackay, D., W. Y. Shiu, and K. C. Ma, 1992b: Illustrated Handbook of Physical-Chemical Properties and Environmental Fate for Organic Chemicals. Volume II. Polynuclear Aromatic Hydrocarbons, Polychlorinated Dioxins, and Dibenzofurans. Lewis Publishers, Boca Raton.

Mackay, D., W. Y. Shiu, and K. C. Ma, 1993: Illustrated Handbook of Physical-Chemical Properties and Environmental Fate for Organic Chemicals. Volume III. Volatile Organic Chemicals. Lewis Publishers, Boca Raton. 
Mackay, D., W. Y. Shiu, and K. C. Ma, 1995: Illustrated Handbook of Physical-Chemical Properties and Environmental Fate for Organic Chemicals. Volume IV. Oxygen, Nitrogen, and Sulfur Containing Compounds. Lewis Publishers, Boca Raton.

McDonald, C. and H. J. Duncan, 1979: Particle size distribution of metals in the atmosphere of Glasgow. Atmos. Environ. 13, 977-980.

McVeety, B. D., and R. A. Hites, 1988: Atmospheric deposition of polycyclic aromatic hydrocarbons to water surfaces: A mass balance approach. Atmos. Environ., 22, 511-536.

Mészáros, E., T. Barcza, A. Gelencsér, J. Hlavay, Gy. Kiss, Z. Krivácsy, A. Molnár and K. Polyák, 1997: Size distributions of inorganic and organic species in the atmospheric aerosol in Hungary. J. Aerosol Sci. 28, 1163-1175.

Noihan, J., and S. Planton, 1989: A simple parameterization of land surface processes for meteorological models. Mon. Wea. Rev., 117, 536-549.

OECD, 1989: Control of Toxic Substances in the Atmosphere-Asbestos. Organisation for Economic Co-operation and Development Environmental Monographs, No. 20, Ottawa, Canda.

Oke, T. R., 1987: Boundary Layer Climates. Methuen \& Co., London.

Orsini, C. Q., H. C. Kaufmann, K. R. Akselsson, J. W. Winchester, and J. W. Nelson, 1977: Variation of elemental composition with particle size in the St. Louis aerosol. Nuc. Instr. Methods, 142, 91-96.

Paciga, J. J., and R. E. Jervis, 1976: Multielelement size characterization of urban aerosols. Environ. Sci. Technol. 12, 1124-1128.

Padro, J., 1994: Observed characteristics of the dry deposition velocity of $\mathrm{O}_{3}$ and $\mathrm{SO}_{2}$ above a wet deciduous forest. Sci. Total Environ., 146/147, 395-400.

Pankow, J. F., 1987: Review and comparative analysis of the theories on partitioning between the gas and aerosol particulate phases in the atmosphere. Atmos. Environ., 21, 2275-2283.

Panofsky, H. A., and J. A. Dutton, 1984: Atmospheric Turbulence, Models and Methods for Engineering Applications. John Wiley \& Sons, New York.

Paterson, S., D. Mackay, E. Bacci, and D. Calamari, 1991: Correlation of the equilibrium and kinetics of leaf-air exchange of hydrophobic organic chemicals. Environ. Sci. Technol., 25, 866-871.

Pinto, J. P., R. K. Stevens, R. D. Willis, R. Kellogg, Y. Mamane, J. Novak, J. Santroch, I. Benes, J. Lenicek, and V. Bures, 1998: Czech air quality monitoring and receptor modeling study. Environ. Sci. Technol., 32, 843-854. 
Pirrone, N., G. Glinsborn, and G. J. Keeler, 1995a: Ambient levels and dry deposition fluxes of mercury to Lakes Huron, Erie, and St. Clair. Water, Air, Soil Pollut., 80, 179-188.

Pirrone, N., G. J. Keeler, and T. M. Holsen, 1995b: Dry deposition of trace elements to Lake Michigan: A hybrid-receptor deposition modeling approach. Environ. Sci. Technol. 29, 21122122.

Pirrone, N., G. J. Keeler, and I. Allegrini, 1996: Particle size distributions of atmospheric mercury in urban and rural areas. J. Aerosol. Sci., 27, S13-S14.

Pleim, J. E., 1999: Modeling stomatal response to atmospheric humidity. Preprints, 13th Symposium on Boundary Layers and Turbulence, pp. 291-294, Amer. Meteorol. Soc, Boston.

Pleim, J. E., A. Venkatram, and R. Yamartino, 1984: ADOM/TADAP Model Development Program. Volume 4. The Dry Deposition Module, ERT Document No. P-B980-520, Environmental Research \& Technology, Inc., Concord, Massachusetts.

Poster, D. L., and J. E. Baker, 1996: Influence of submicron particles on hydrophobic organic contaminants in precipitation. 2. Scavenging of polycyclic aromatic hydrocarbons by rain. Environ. Sci. Technol., 30, 349-354.

Reid, R. C., J. M. Prausnitz, and B. E. Poling, 1987: The Properties of Gases and Liquids. McGraw-Hill, New York.

Riederer, M., 1990: Estimating partitioning and transport of organic chemicals in the foliage/atmosphere system: Discussion of a fugacity-based model. Environ. Sci. Technol., 24, 829-837.

Rizzio, E., G. Giaveri, D. Arginelli, L. Gini, A. Profumo, and M. Gallorini, 1999: Trace elements total content and particle sizes distribution in the air particulate matter of a rural-residential area in north Italy investigated by instrumental neutron activation analysis. Sci. Tot. Environ., 226, 47-56.

Rosenberg, N. J., B. L. Blaine, and S. B. Verma, 1983: Microclimate: The Biological Environment. Wiley-Interscience, New York.

Rutter, A. J., 1975: The hydrological cycle in vegetation. In Vegetation and the Atmosphere, Volume 1, edited by J. L. Monteith, pp. 111-154, Academic Press, New York.

Sakai, R. K., D. R. Fitzjarrald, and K. E. Moore, 1997: Detecting leaf area and surface resistance during transition seasons. Agric. For. Meteorol., 84, 273-284.

Sander, R., 1999: Compilation of Henry's Law Constants for Inorganic and Organic Species of Potential Importance in Environmental Chemistry, Version 3. http://www.mpchmainz.mpg.de/ sander/res/henry.html. 
Sellers, P. J., R. E. Dickinson, D. A. Randall, A. K. Betts, F. G. Hall, J. A. Berry, G. J. Collatz, A. S. Denning, H. A. Mooney, C. A. Nobre, N. Sato, C. B. Field, and A. Henderson-Sellers, 1997: Modeling the exchanges of energy, water, and carbon between continents and the atmosphere. Science, 275, 502-509.

Schwede, D. B., and J. O. Paumier, 1997: Sensitivity of the Industrial Source Complex Model to input deposition parameters. J. Appl. Meteorol., 36, 1096-1106.

Scott, B. C, 1981: Modeling of atmospheric wet deposition. In Atmospheric Pollutants in Natural Waters, edited by S. J. Eisenreich, pp. 3-21, Ann Arbor Science, Ann Arbor.

Seinfeld, J. H., and S. N. Pandis, 1998: Atmospheric Chemistry and Physics, from Air Pollution to Climate Change. John Wiley \& Sons, New York.

Shannon, J. D., and E. C. Voldner, 1995: Modeling atmospheric concentrations of mercury and deposition to the Great Lakes. Atmos. Environ., 29, 1649-1661.

Slinn, W. G. N., 1977: Some approximations of the wet and dry removal of particles and gases from the atmosphere. Water, Air, Soil Pollut., 7, 513-543.

Slinn, W. G. N., 1982: Predictions for particle deposition to vegetative canopies. Atmos. Environ., 16, 1785-1794.

Slinn, S. A., and W. G. N. Slinn, 1980: Predictions for particle deposition on natural waters. Atmos. Environ., 14, 1013-1016.

Slinn, W. G. N., L. Hasse, B. B. Hicks, A. W. Hogan, D. Lal, P. S. Liss, K. O. Munnich, G. A. Sehmel, and O. Vittori, 1978: Some aspects of the transfer of atmospheric trace constituents past the air-sea interface. Atmos. Environ., 12, 2055-2087.

Sommar, J., O. Lindqvist, and D. Strömberg, 2000: Distribution equilibrium of mercury (II) chloride between water and air applied to flue gas scrubbing. J. Air \& Waste Manage. Assoc., 50, 1663-1666.

Spicer, C. W., A. J. Pollack, T. J. Kelly, and M. Ramamurthi, 1993: A Literature Review of Atmospheric Transformation Products of the Clean Air Act Title III Hazardous Air Pollutants. EPA/600/R-94/088, U. S. Environmental Protection Agency, Research Triangle Park..

Welke, B., K. Ettlinger, and M. Riederer, 1998: Sorption of volatile organic chemicals in plant surfaces. Environ. Sci. Technol., 32, 1099-1104.

Wesely, M. L., 1979: Relationships between dry deposition rates and concentrations at heights of $50 \mathrm{~m}$ to $200 \mathrm{~m}$. In Control of Emissions from Stationary Combustion Sources: Pollutant Detection and Behavior in the Atmosphere, AIChE Symposium Series, 75, 181-186. 
Wesely, M. L., 1989: Parameterization of surface resistances to gaseous dry deposition in regional-scale numerical models. Atmos. Environ., 23, 1293-1304.

Wesely, M. L., D. R. Cook, R. L. Hart, and R. E. Speer, 1985: Measurement and parameterization of particulate sulfur dry deposition over grass. J. Geophys. Res., 90, 2131-2143. 


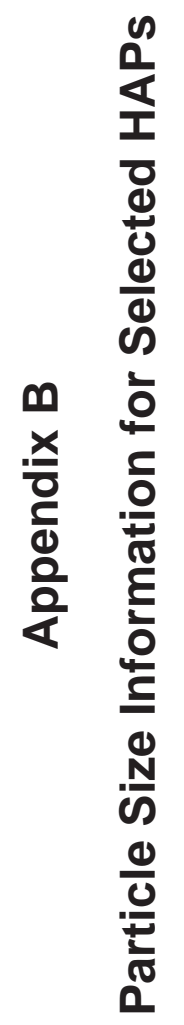




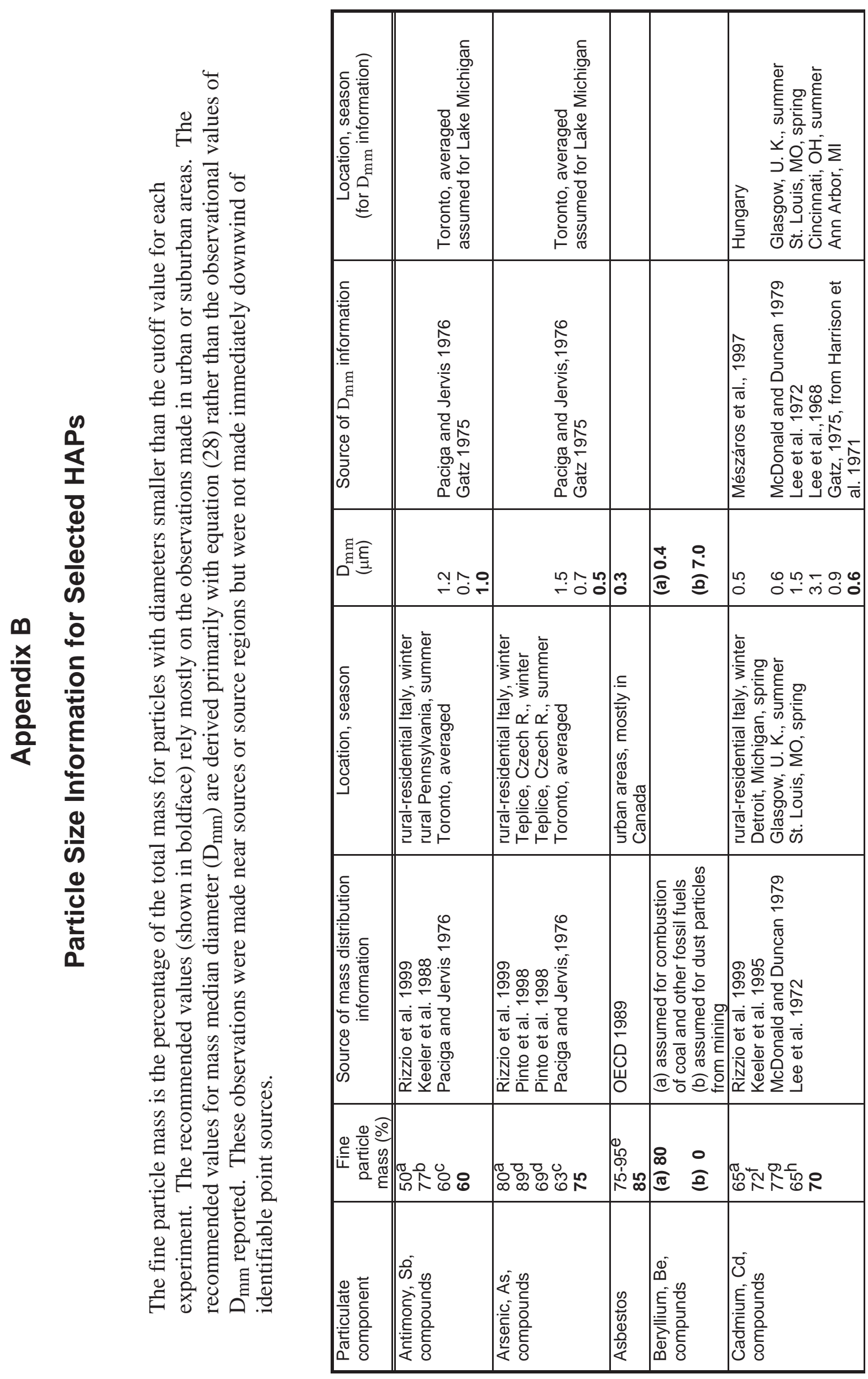




\begin{tabular}{|c|c|c|c|c|c|c|c|c|}
\hline 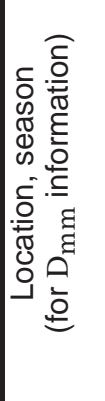 & 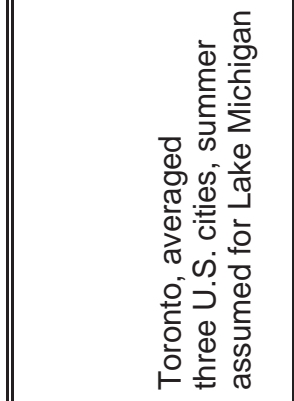 & 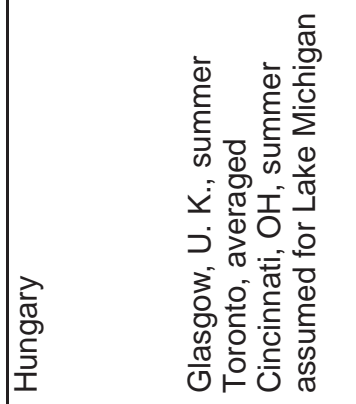 & 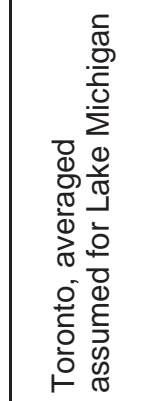 & & & & & \\
\hline 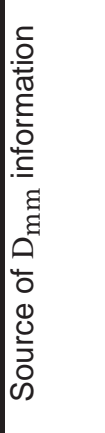 & 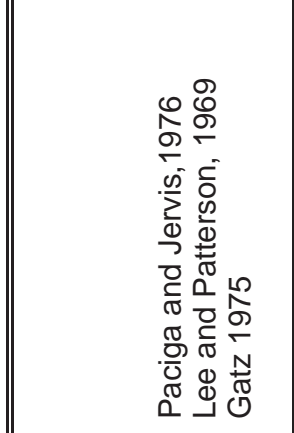 & 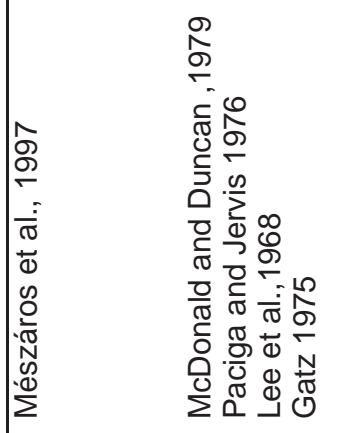 & 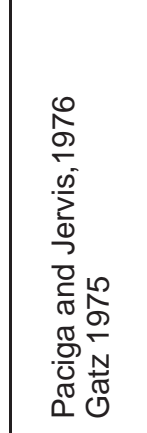 & & & & & \\
\hline 伺 & تَ & ت & 임욤용 & it & ب & & & \\
\hline 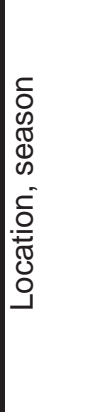 & 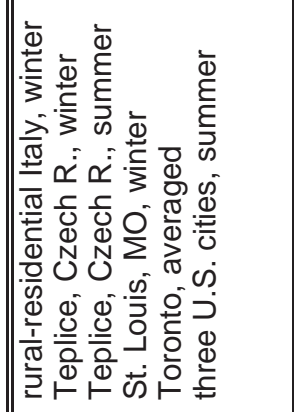 & 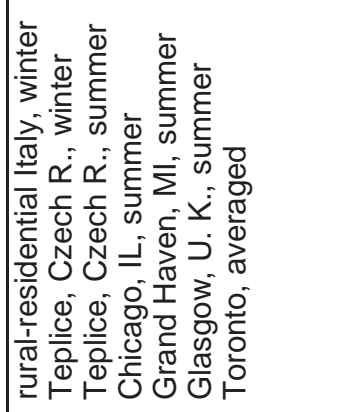 & 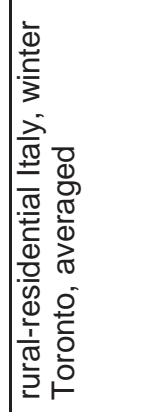 & & & & & \\
\hline 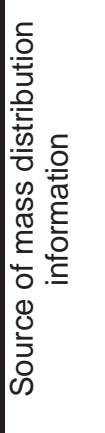 & 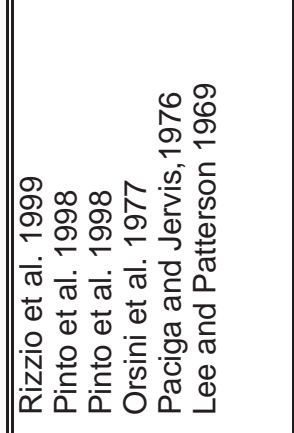 & 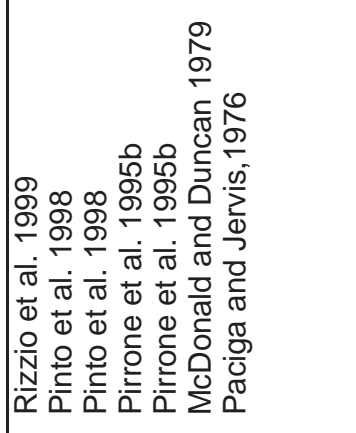 & 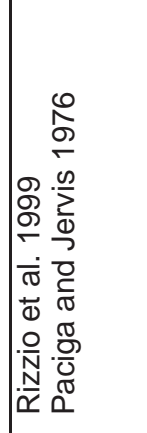 & 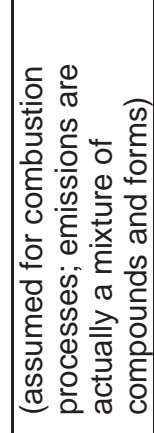 & 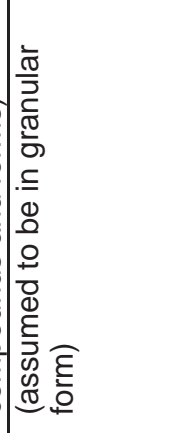 & 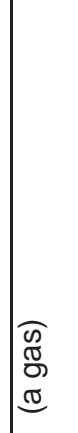 & 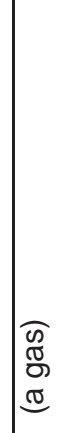 & 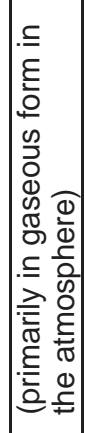 \\
\hline 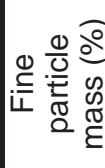 & 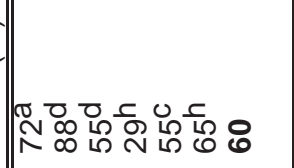 & 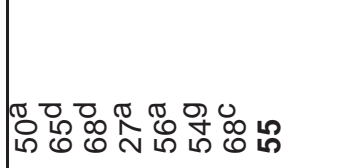 & $\frac{\pi}{\pi} 0$ & ঃ & 0 & $\frac{\pi}{2}$ & $\frac{\pi}{2}$ & $\frac{\pi}{\Omega}$ \\
\hline 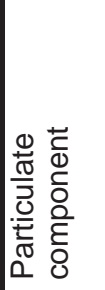 & 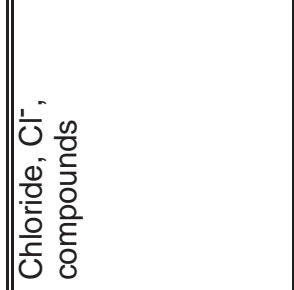 & 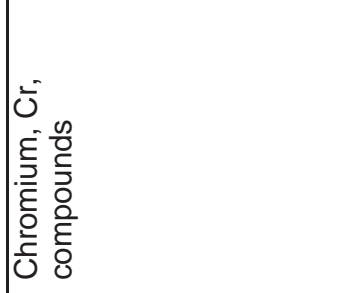 & 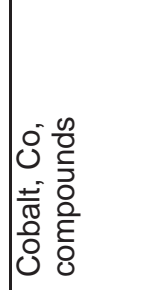 & 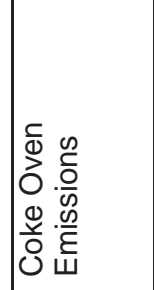 & 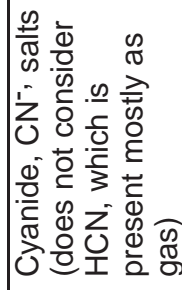 & 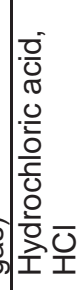 & 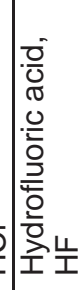 & 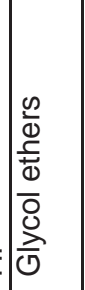 \\
\hline
\end{tabular}




\begin{tabular}{|c|c|c|c|c|}
\hline 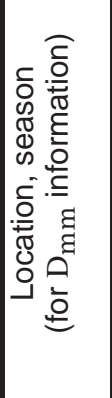 & 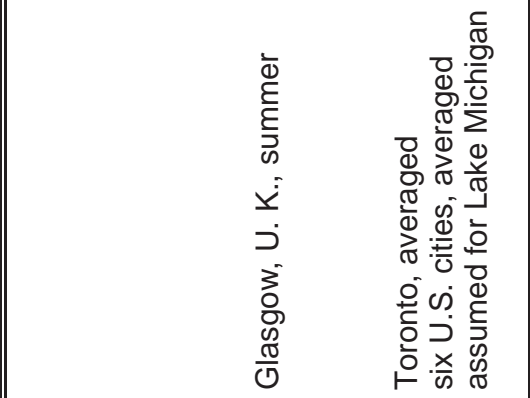 & 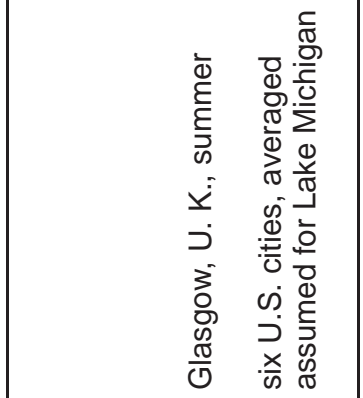 & $\begin{array}{l}\sum \\
\bar{\Sigma} \\
\overline{\overline{0}} \\
\overline{\frac{1}{0}} \\
0\end{array}$ & \\
\hline 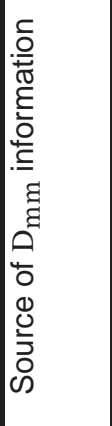 & 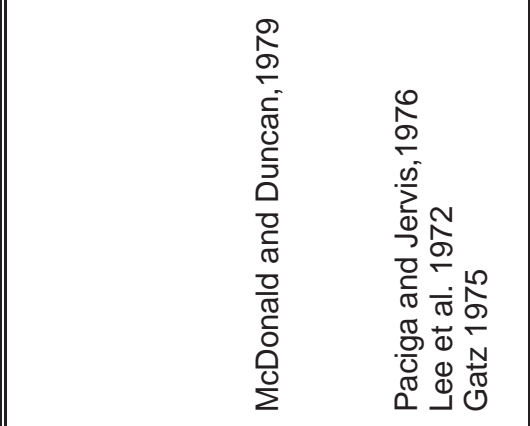 & 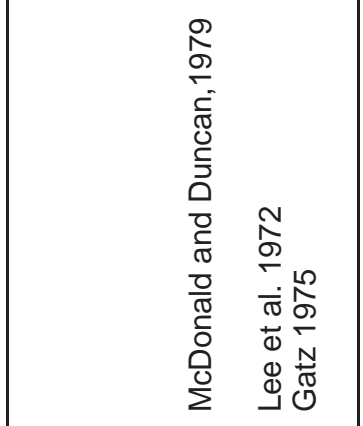 & 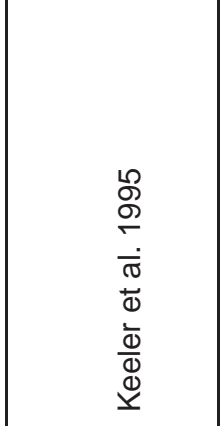 & \\
\hline 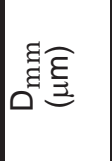 & 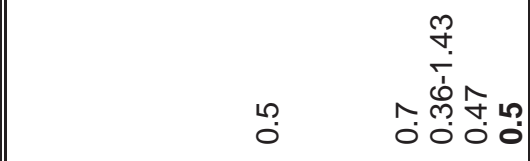 & 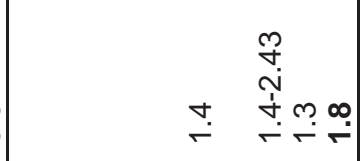 & 苗 & - \\
\hline 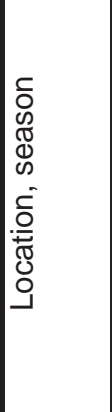 & 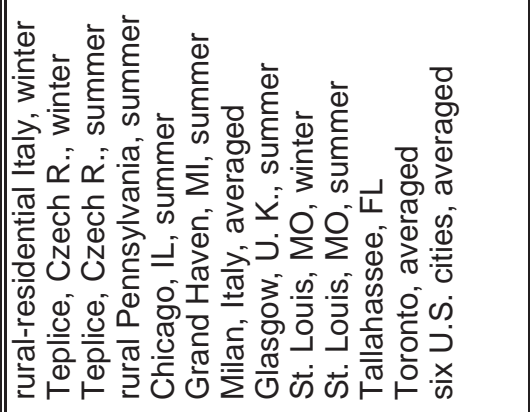 & 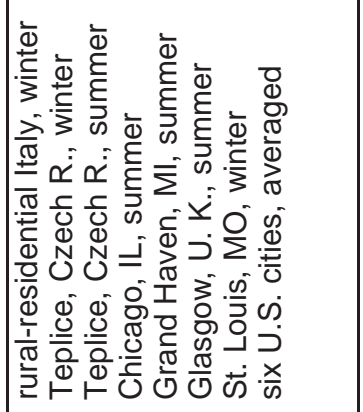 & 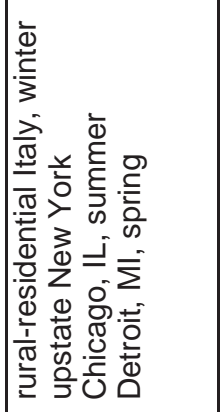 & \\
\hline 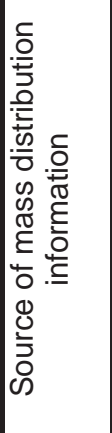 & 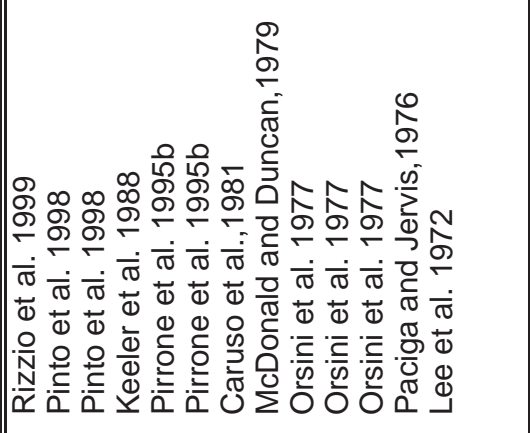 & 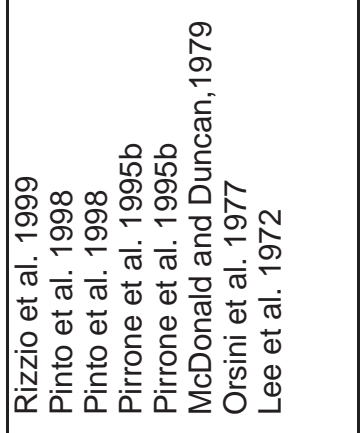 & 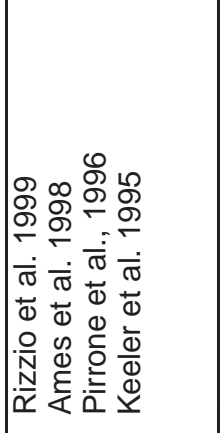 & 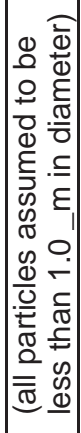 \\
\hline 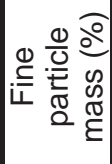 & 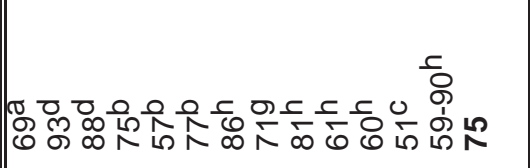 & 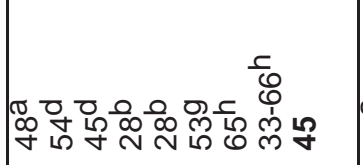 & 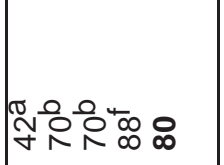 & 욱 \\
\hline 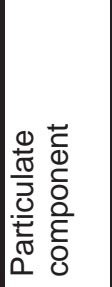 & 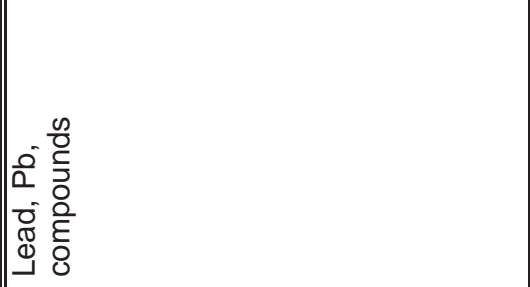 & 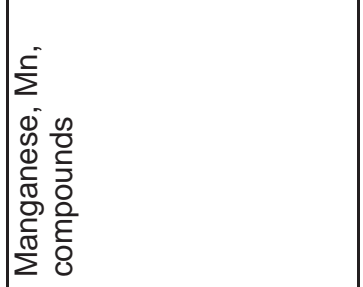 & 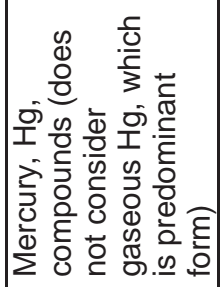 & 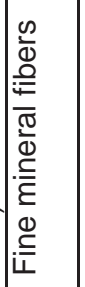 \\
\hline
\end{tabular}




\begin{tabular}{|c|c|c|c|c|c|c|}
\hline 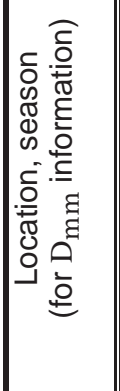 & 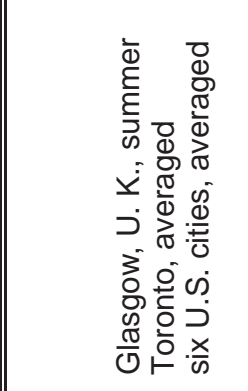 & 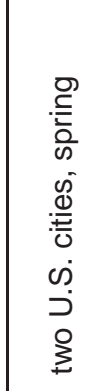 & & & & \\
\hline 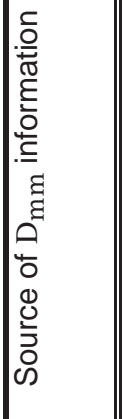 & 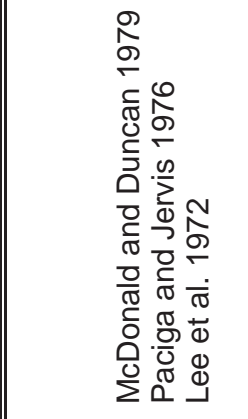 & 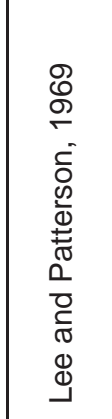 & & & & \\
\hline 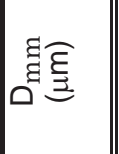 & 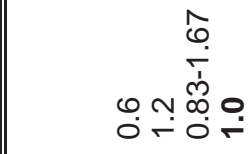 & $\begin{array}{l}\infty N N \\
\end{array}$ & $\overbrace{0}^{\circ}$ & - & -1 & Ȯ \\
\hline 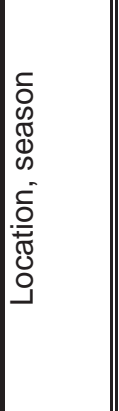 & 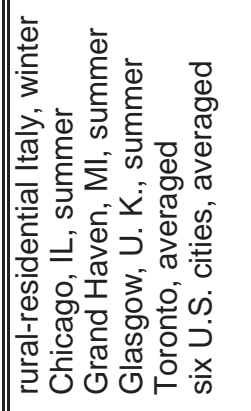 & 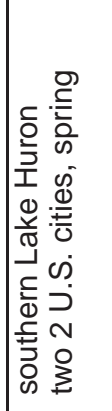 & & 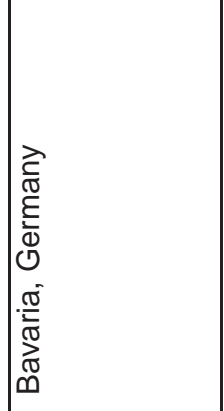 & 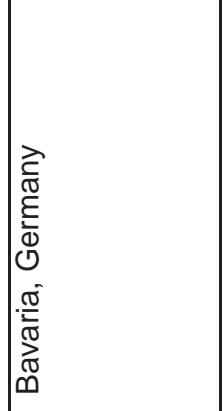 & \\
\hline 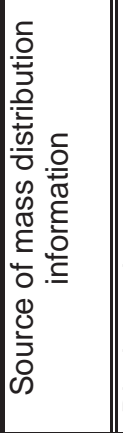 & 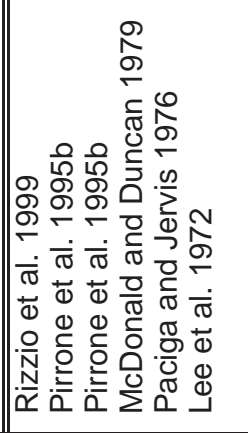 & 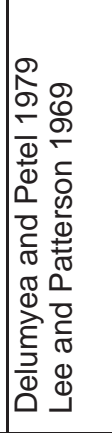 & 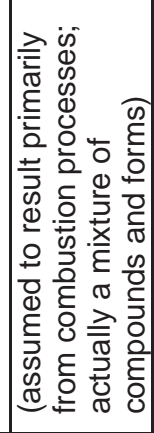 & 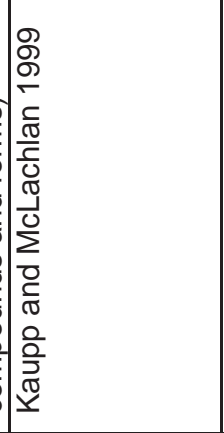 & 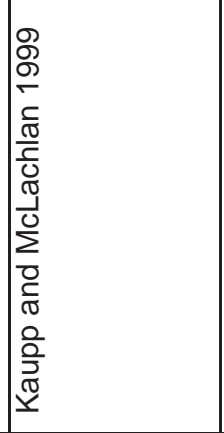 & 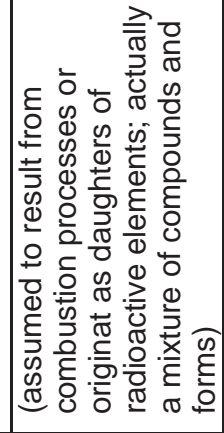 \\
\hline 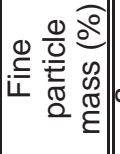 & 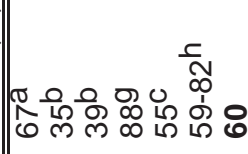 & 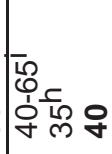 & ৪ & నু & $\begin{array}{l} \\
\sigma \\
\infty \\
\infty \\
\infty \\
\infty\end{array}$ & ঃ \\
\hline 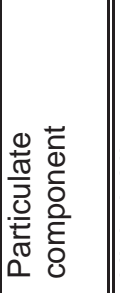 & 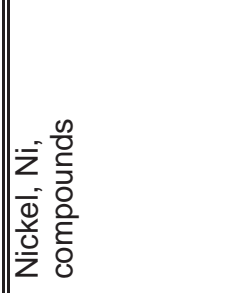 & 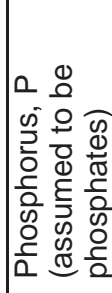 & 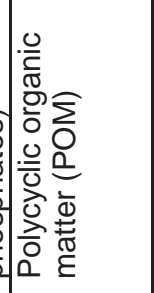 & 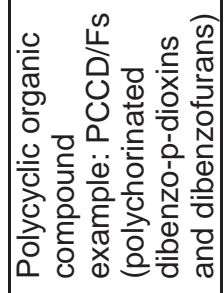 & 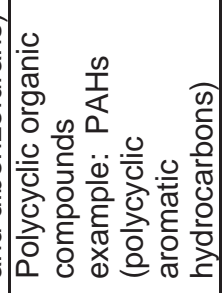 & 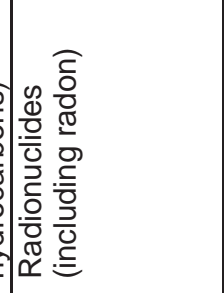 \\
\hline
\end{tabular}




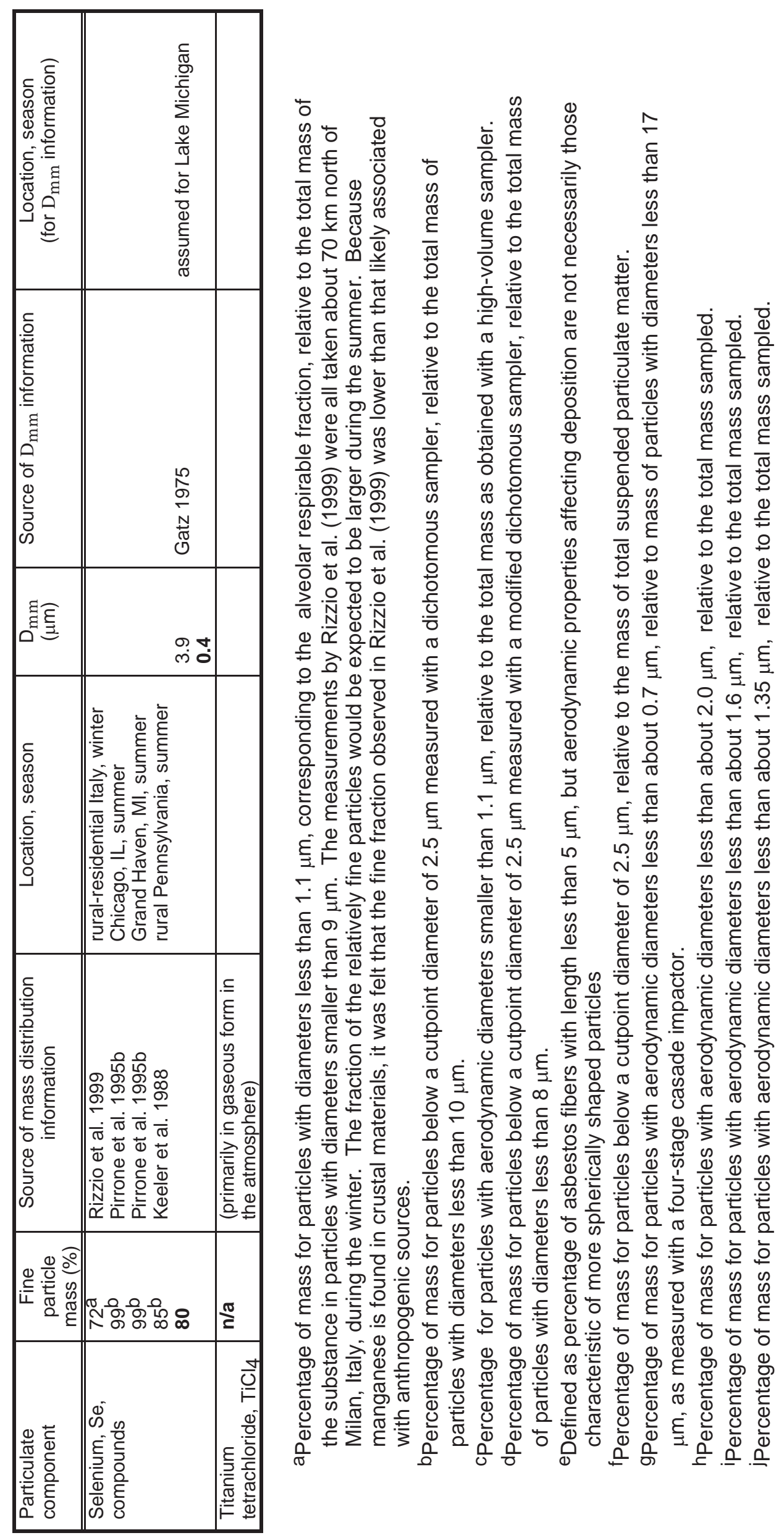

ค่ 


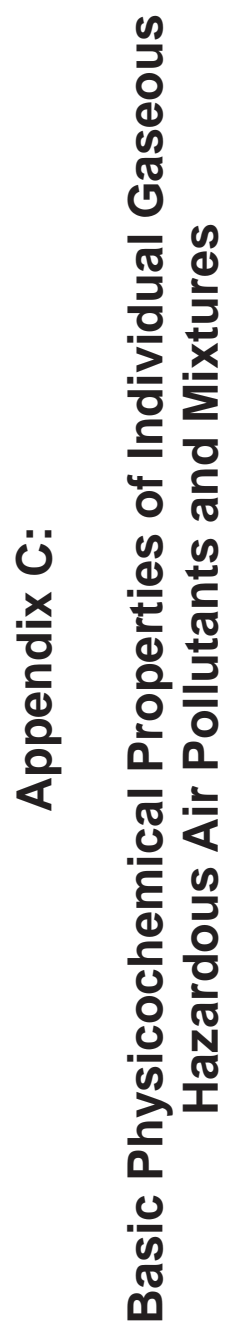




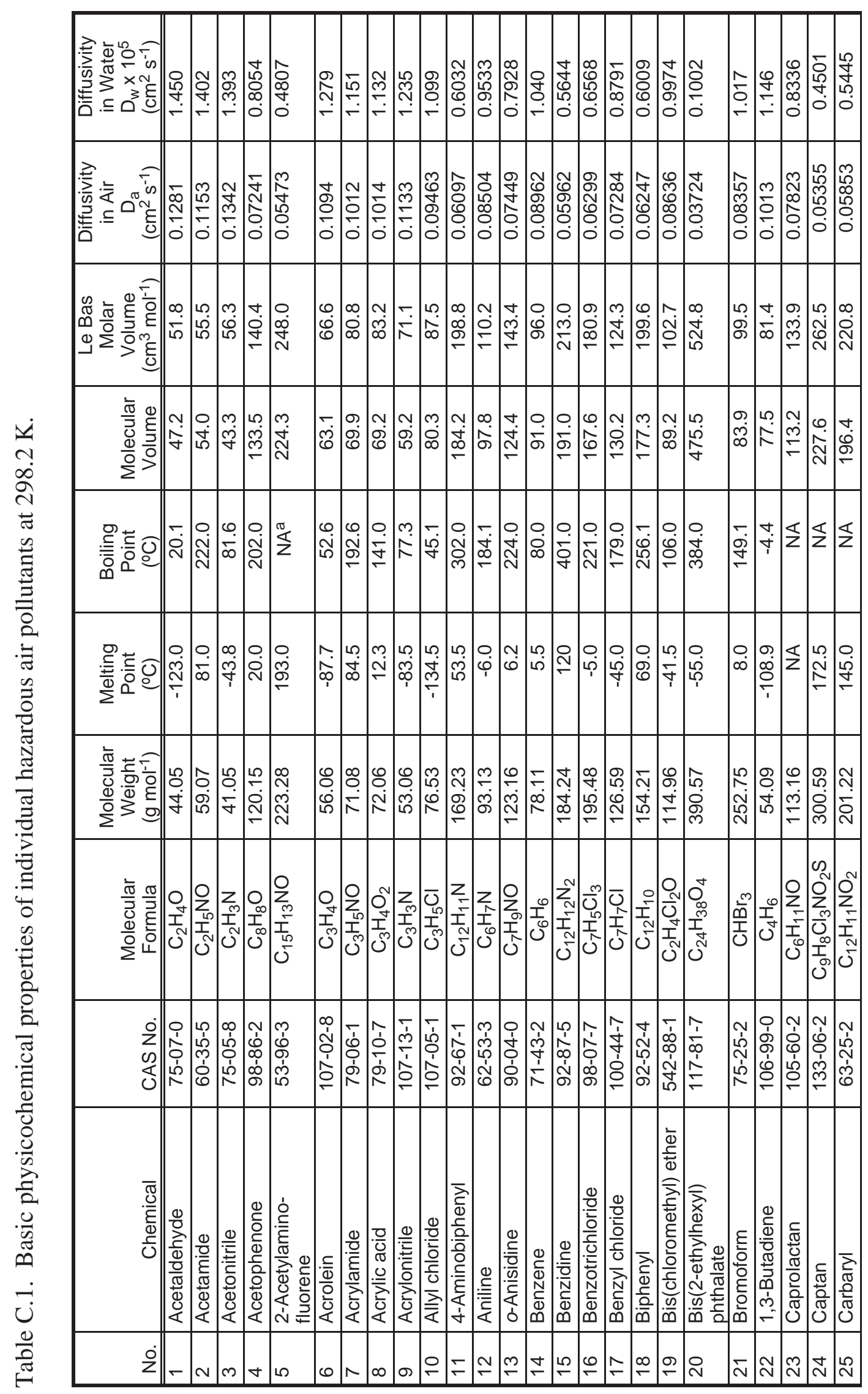




\begin{tabular}{|c|c|c|c|c|c|c|c|c|c|c|c|c|c|c|c|c|c|c|c|c|c|c|c|c|}
\hline 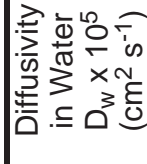 & 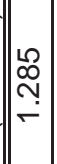 & 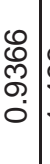 & হু & 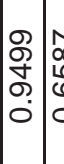 & 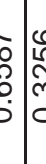 & 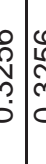 & : & 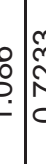 & & 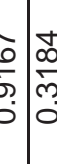 & & & $\underset{⿱}{\stackrel{m}{+}}$ & & 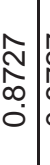 & & $\begin{array}{c}\hat{N} \\
\mathbb{N} \\
\infty \\
0 \\
0\end{array}$ & & & 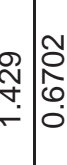 & 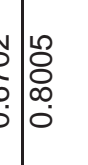 & $\begin{array}{c}\hat{N} \\
\hat{N} \\
0 \\
0 \\
0\end{array}$ & $\begin{array}{l}\bar{c} \\
\bar{p} \\
0 \\
0\end{array}$ & 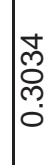 \\
\hline 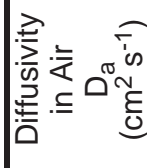 & $\mid$\begin{tabular}{l}
$\infty$ \\
\hdashline \\
0 \\
\hdashline \\
0 \\
0
\end{tabular} & & 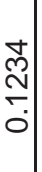 & 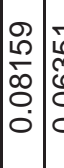 & 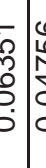 & 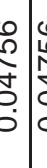 & 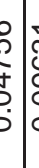 & 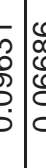 & & 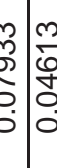 & & \begin{tabular}{l|l} 
& \\
8 & \\
0 \\
0 \\
0 \\
0
\end{tabular} & $\begin{array}{l}0 \\
\text { 응 } \\
\text { O্ণ } \\
0 \\
0\end{array}$ & $\mid \begin{array}{l}n \\
1 \\
0 \\
0 \\
0 \\
0\end{array}$ & $\begin{array}{l}\tilde{N} \\
\tilde{N} \\
\vdots \\
0 \\
0\end{array}$ & 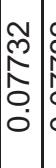 & 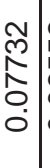 & 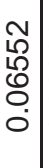 & 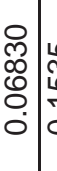 & 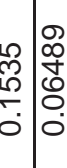 & 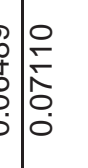 & 竞 & 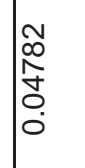 & $\begin{array}{l}0 \\
0 \\
0 \\
0 \\
0 \\
0 \\
0\end{array}$ \\
\hline 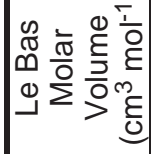 & $\mid \begin{array}{l}0 \\
0 \\
0 \\
0\end{array}$ & $\begin{array}{l}\stackrel{N}{ } \\
\stackrel{m}{\sigma}\end{array}$ & \begin{tabular}{|c|}
$\hat{a}$ \\
$\infty$ \\
$o$ \\
\end{tabular} & 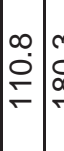 & & & & & & & & $\begin{array}{l}m \\
\\
\tilde{\sigma}\end{array}$ & 文 & & $\begin{array}{c}0 \\
\stackrel{2}{N} \\
\stackrel{2}{-}\end{array}$ & 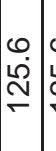 & $\begin{array}{l}0 \\
\stackrel{2}{N} \\
\stackrel{-}{-}\end{array}$ & & 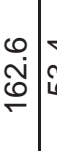 & 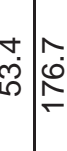 & $=\frac{d}{d}$ & 赵 & 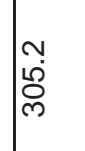 & $\begin{array}{l}\text { va } \\
\text { N } \\
\text { ले }\end{array}$ \\
\hline 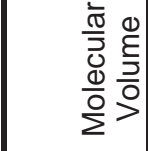 & $\frac{\sim}{6}$ & ه. & $\begin{array}{l}\mathscr{g} \\
\dot{f}\end{array}$ & 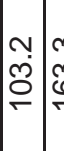 & 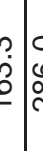 & 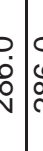 & $\begin{array}{lll}0 & \\
0 \\
0 \\
0\end{array}$ & : & & P. & & 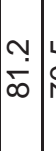 & $\stackrel{n}{R}$ & $\begin{array}{l}\tilde{x} \\
\mathscr{\delta} \\
\mathscr{\delta}\end{array}$ & $\begin{array}{l}0 \\
\\
\underline{T}\end{array}$ & $\begin{array}{l}0 \\
\dot{1} \\
\\
\end{array}$ & $\begin{array}{l}0 \\
\dot{1} \\
\bar{T}\end{array}$ & \begin{tabular}{l}
- \\
$\dot{0}$ \\
\hdashline
\end{tabular} & 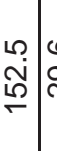 & 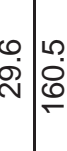 & 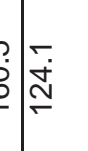 & ळ্் & $\begin{array}{l}\infty \\
\infty \\
\infty \\
\infty \\
\sim\end{array}$ & $\frac{⿱ 亠 䒑}{\frac{\pi}{m}}$ \\
\hline 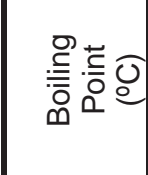 & $\mid \begin{array}{l}0 \\
\dot{\theta} \\
\dot{q}\end{array}$ & $\begin{array}{l}\infty \\
\dot{\rho} \\
\stackrel{1}{*}\end{array}$ & $\begin{array}{l}0 \\
\dot{0} \\
\vdots \\
.\end{array}$ & $\mid \begin{array}{c}0 \\
\dot{j} \\
\stackrel{1}{N} \\
N\end{array}$ & & $\mathbb{Z} \Sigma$ & $z$ & 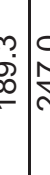 & & $=$ & 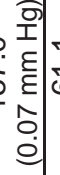 & $\div$ & مீ & 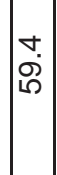 & $\begin{array}{l}0 \\
\dot{\sigma} \\
\frac{5}{-}\end{array}$ & 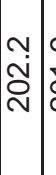 & @) & $\begin{array}{l}0 \\
\infty \\
\infty \\
\infty\end{array}$ & 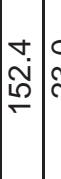 & 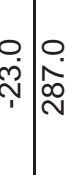 & $\begin{array}{l}0 \\
0 \\
0 \\
0\end{array}$ & $\left|\begin{array}{rr}0 & \overline{0} \\
0 & 0 \\
0 & \varepsilon \\
0 & \varepsilon \\
0 & 0 \\
0 \\
0\end{array}\right|$ & $\frac{1}{z}$ & $\begin{array}{l}0 \\
\text { o } \\
\text { ले }\end{array}$ \\
\hline 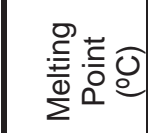 & 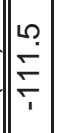 & 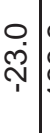 & $\begin{array}{l}\infty \\
\infty \\
m \\
\\
\end{array}$ & 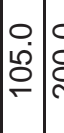 & bे & 2 & 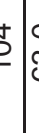 & 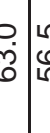 & & 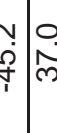 & & $\mid \begin{array}{l}0 \\
0 \\
\tilde{n} \\
0 \\
1\end{array}$ & مִ & \begin{tabular}{l}
0 \\
\hdashline \\
$\dot{m}$ \\
1
\end{tabular} & 悉 & 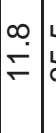 & $\mid$ & & 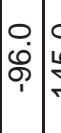 & 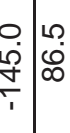 & $\dot{b}$ & مُ & ○ & $\begin{array}{l}0 \\
\text { p. } \\
\text { p. }\end{array}$ \\
\hline 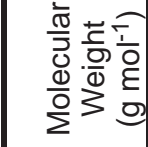 & 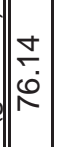 & 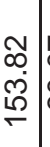 & $\begin{array}{l}\hat{0} \\
\dot{0}\end{array}$ & 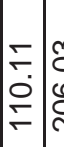 & 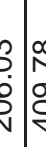 & $\begin{array}{ll}\infty & 0 \\
\vdots \\
\vdots \\
\sigma\end{array}$ & 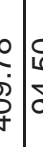 & 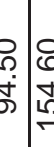 & 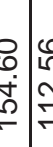 & 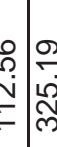 & & 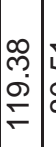 & 占 & $\mid \begin{array}{l}1 \\
10 \\
\infty \\
\infty \\
\infty\end{array}$ & 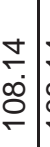 & 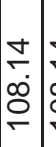 & $\mid$\begin{tabular}{l}
0 \\
\hdashline \\
$\infty$ \\
0 \\
0
\end{tabular} & $\mid \begin{array}{l}\infty \\
\frac{0}{0} \\
10 \\
1\end{array}$ & 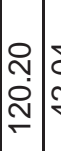 & 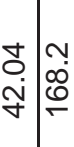 & 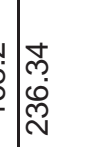 & 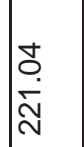 & $\begin{array}{l}\infty \\
0 \\
\infty \\
\infty \\
\infty\end{array}$ & 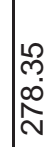 \\
\hline 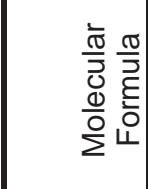 & $\mid$ & তั & ญ. & 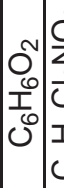 & 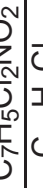 & 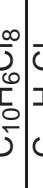 & 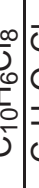 & כָ & 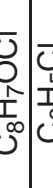 & 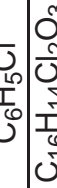 & & $\frac{m}{0}$ & 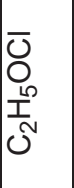 & $\begin{array}{l}\overline{0} \\
15 \\
T^{J} \\
0\end{array}$ & $\begin{array}{l}0 \\
0 \\
\frac{\infty}{1} \\
0 \\
0\end{array}$ & 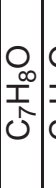 & $\mid \begin{array}{c}0 \\
0 \\
1 \\
1 \\
0 \\
0\end{array}$ & 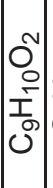 & & 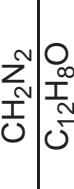 & 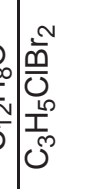 & $\begin{array}{l}N_{0} \\
0 \\
0 \\
1 \\
I \\
0 \\
0\end{array}$ & 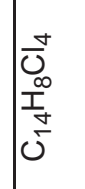 & 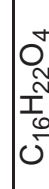 \\
\hline $\begin{array}{l}0 \\
2 \\
0 \\
0 \\
0\end{array}$ & 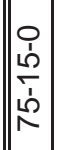 & 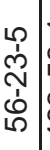 & 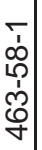 & 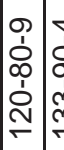 & 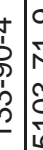 & 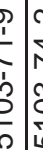 & 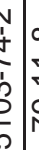 & 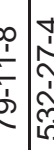 & 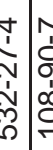 & 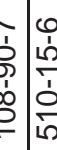 & & $\begin{array}{l}m \\
0 \\
0 \\
0 \\
1 \\
0 \\
0\end{array}$ & 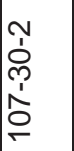 & 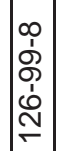 & 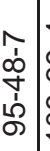 & 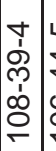 & $\mid \begin{array}{l}1 \\
1 \\
f \\
f \\
\vdots \\
0 \\
0 \\
0\end{array}$ & 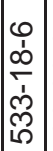 & 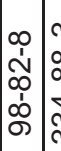 & 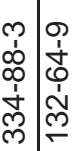 & 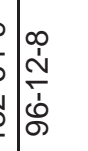 & $\begin{array}{l}\hat{1} \\
\hat{n} \\
\hat{j} \\
\dot{\sigma}\end{array}$ & $\begin{array}{l}0 \\
1 \\
1 \\
1 \\
1 \\
\\
\end{array}$ & \begin{tabular}{l}
$N$ \\
\multirow{2}{*}{} \\
$\hat{N}$ \\
$\dot{y}$ \\
$\infty$
\end{tabular} \\
\hline $\begin{array}{l}\overline{\bar{J}} \\
\frac{0}{E} \\
\bar{D} \\
\bar{U} \\
\end{array}$ & 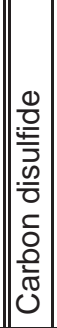 & 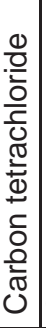 & 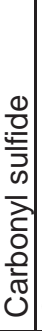 & 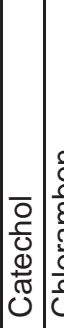 & 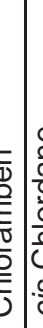 & 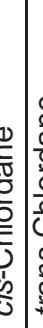 & 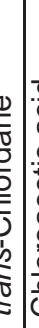 & 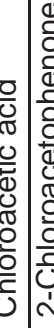 & 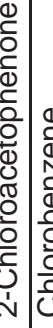 & 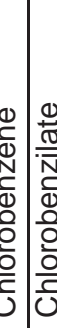 & & $\begin{array}{l} \\
\frac{\varepsilon}{2} \\
\frac{0}{0} \\
\frac{0}{0} \\
\frac{0}{0} \\
0\end{array}$ & 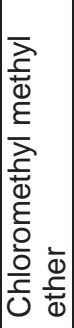 & 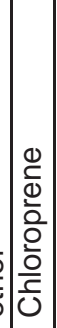 & $\begin{array}{l}\overline{0} \\
\mathscr{d} \\
\underline{d} \\
\vdots \\
\vdots\end{array}$ & $\begin{array}{l}\overline{0} \\
\dot{d} \\
\mathbb{d} \\
\bar{\nu} \\
1 \\
\Sigma\end{array}$ & 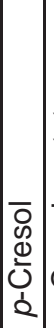 & 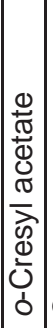 & 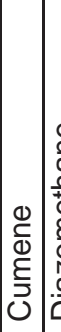 & 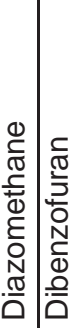 & 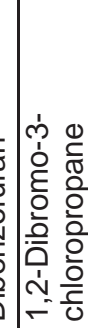 & 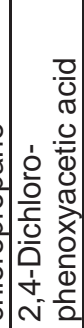 & 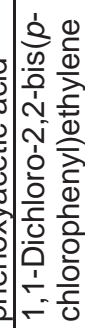 & 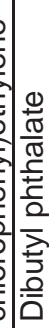 \\
\hline in & $\stackrel{\leftrightarrow}{\stackrel{2}{*}}$ & $\hat{N}$ & & $\mathbb{N}$ & $\vec{n}$ & $\bar{m}$ & va & 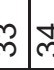 & & 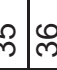 & & $\hat{m}$ & 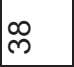 & প্ল & 8 & 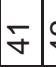 & | & 隹 & $\forall$ & Lে & f & $\stackrel{\wp}{\&}$ & 守 & in \\
\hline
\end{tabular}




\begin{tabular}{|c|c|c|c|c|c|c|c|c|c|c|c|c|c|c|c|c|c|c|c|c|c|c|c|}
\hline 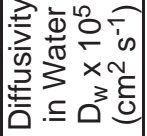 & $\mid \begin{array}{c}1 \\
0 \\
0 \\
\infty \\
0 \\
0\end{array}$ & 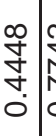 & 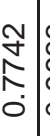 & 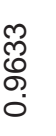 & 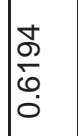 & $\mid \begin{array}{c}\mathbb{N} \\
\hat{L} \\
\infty \\
0 \\
0\end{array}$ & $\mid$ & 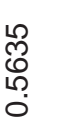 & $\mid$ & & $\mid$\begin{tabular}{c}
$\mathscr{N}$ \\
\multirow{N}{N}{} \\
0 \\
0
\end{tabular} & $\begin{array}{l}n \\
0 \\
0 \\
0 \\
0\end{array}$ & 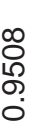 & \begin{tabular}{l}
$\infty$ \\
$\infty$ \\
$\infty$ \\
\hdashline \\
\end{tabular} & 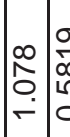 & 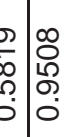 & 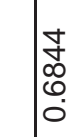 & 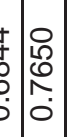 & $\begin{array}{l}\infty \\
\infty \\
0 \\
⿱ 乛 \\
0 \\
0\end{array}$ & 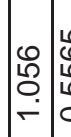 & 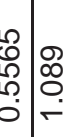 & & $\mid \begin{array}{l}m \\
m \\
\infty \\
\infty \\
0 \\
0\end{array}$ \\
\hline 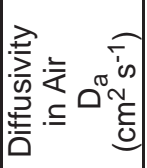 & $\left|\begin{array}{c}0 \\
\mathbb{N} \\
\text { N } \\
0 \\
0 \\
0\end{array}\right|$ & 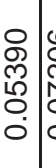 & 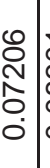 & $\begin{array}{l}\text { Dे } \\
\text { o } \\
0 \\
0 \\
0\end{array}$ & $\begin{array}{l}\hat{N} \\
\tilde{O} \\
0 \\
0\end{array}$ & $\mid \begin{array}{l}+ \\
\frac{\pi}{0} \\
\infty \\
0 \\
0\end{array}$ & 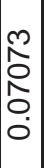 & 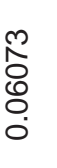 & $\begin{array}{l}\mathcal{1} \\
\text { N్} \\
\hat{\wp} \\
0 \\
0\end{array}$ & $\begin{array}{l}\stackrel{2}{N} \\
\text { N } \\
\text { Oొ } \\
0 \\
0\end{array}$ & $\mid$\begin{tabular}{c}
$\mp$ \\
\hdashline \\
\hdashline \\
0 \\
0
\end{tabular} & 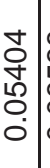 & 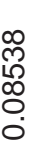 & $\begin{array}{l}\stackrel{8}{2} \\
\infty \\
\infty \\
0 \\
0\end{array}$ & 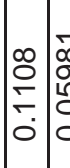 & 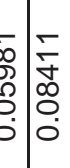 & $\begin{array}{l}\overline{1} \\
0 \\
0 \\
0 \\
0 \\
0\end{array}$ & 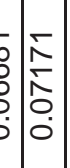 & $\begin{array}{l}10 \\
\infty \\
0 \\
0 \\
0 \\
0\end{array}$ & 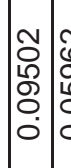 & 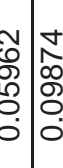 & 음 & $\mid \begin{array}{ll}2 \\
0 \\
0 \\
0\end{array}$ \\
\hline 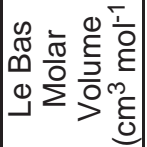 & 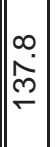 & 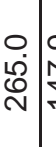 & $\begin{array}{l}\stackrel{g}{*} \\
\dot{f} \\
\leftarrow\end{array}$ & $\begin{array}{l}+ \\
\infty \\
\infty\end{array}$ & ஜூ & No & 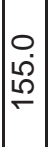 & $\frac{m}{m}$ & 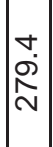 & $\begin{array}{l}0 \\
\text { in } \\
\text { N }\end{array}$ & & 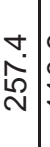 & $\begin{array}{l}\stackrel{0}{0} \\
\stackrel{0}{+}\end{array}$ & $\hat{\infty}$ & சें & $\begin{array}{l}\text { : } \\
\dot{S}\end{array}$ & $\stackrel{\substack{N\\
}}{\frac{1}{n}}$ & 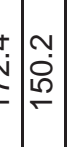 & 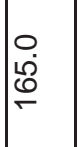 & 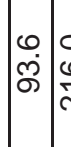 & \begin{tabular}{c|c}
0 \\
\hdashline \\
& 0 \\
& $\infty$ \\
$\infty$
\end{tabular} & & 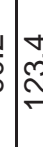 \\
\hline 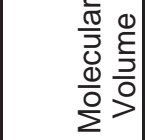 & 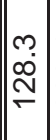 & 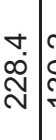 & $\begin{array}{l}N \\
\grave{s} \\
\text { ma } \\
\end{array}$ & ळ. & Гூ & $\mid$\begin{tabular}{l}
$\infty$ \\
$\infty$ \\
0 \\
\hdashline
\end{tabular} & $\begin{array}{l}0 \\
\dot{m} \\
-\end{array}$ & ז. & $\begin{array}{c}m \\
\dot{d} \\
\sim \\
N\end{array}$ & Wึ & $\begin{array}{l}0 \\
\infty \\
m \\
-1 \\
\end{array}$ & $\begin{array}{l}\circ \\
\text { N̦} \\
\text { N }\end{array}$ & 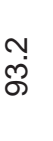 & 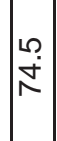 & मे & 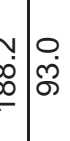 & مُ & 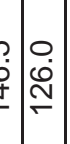 & 守 & 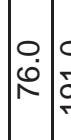 & 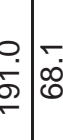 & סृ & $\underset{c}{c}$ \\
\hline 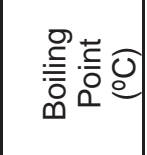 & 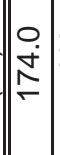 & $\S$ & 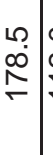 & $\stackrel{\stackrel{O}{\mathrm{i}}}{\mathrm{E}}$ & 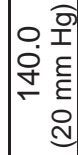 & $\begin{array}{l}\infty \\
\infty \\
\infty \\
\infty \\
\infty\end{array}$ & $\begin{array}{l}0 \\
\infty \\
0 \\
\infty \\
N\end{array}$ & $\frac{O}{\text { N̦ }}$ & $\Sigma$ & $\Sigma$ & 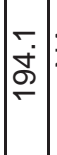 & $\Sigma$ & $\begin{array}{l}\stackrel{0}{r} \\
\stackrel{0}{0} \\
\end{array}$ & $\begin{array}{l}0 \\
\tilde{N} \\
\underline{0} \\
\\
\end{array}$ & 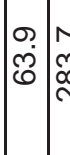 & : & 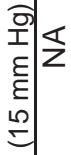 & 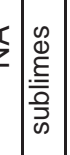 & 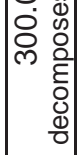 & 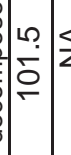 & $\underset{\substack{0 \\
\Sigma}}{\frac{0}{\pi}}$ & & ১ \\
\hline 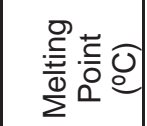 & 泠 & 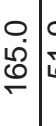 & ?ִ & $\Sigma$ & $\Sigma$ & $\begin{array}{l}0 \\
\infty \\
\stackrel{\infty}{a}\end{array}$ & $\begin{array}{l}0 \\
\dot{\mathfrak{j}} \\
\stackrel{1}{1}\end{array}$ & $\begin{array}{l}0 \\
0 \\
0 \\
0 \\
1\end{array}$ & $\begin{array}{l}0 \\
\stackrel{1}{2} \\
\underline{m} \\
\end{array}$ & 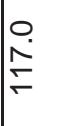 & ì & $\mid$ & @्. & $\mid \begin{array}{l}\dot{0} \\
\dot{0} \\
\dot{0} \\
1\end{array}$ & 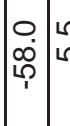 & ָْ & $\mid$ & 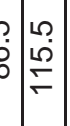 & $\frac{0}{\frac{1}{r}}$ & $\underset{c}{\infty}$ & 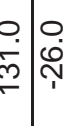 & $\begin{array}{l}0 \\
0 \\
10 \\
10 \\
1\end{array}$ & ? \\
\hline
\end{tabular}

$\frac{7}{\frac{7}{2}}$

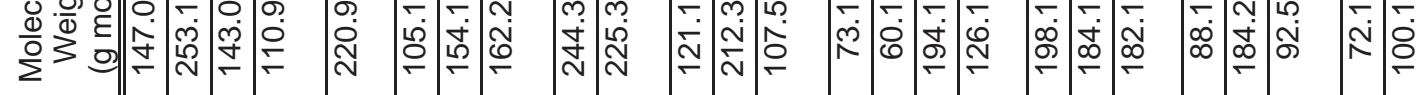

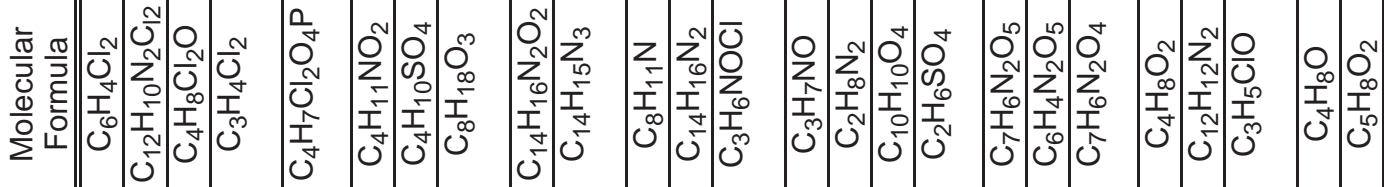

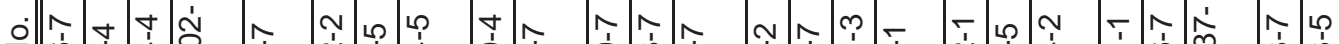

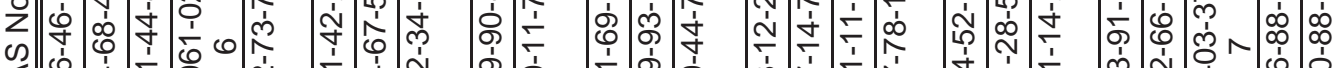

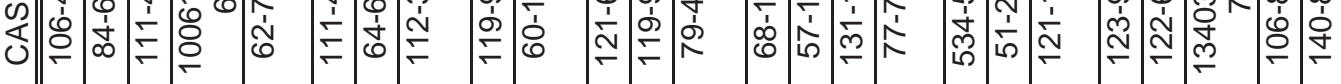

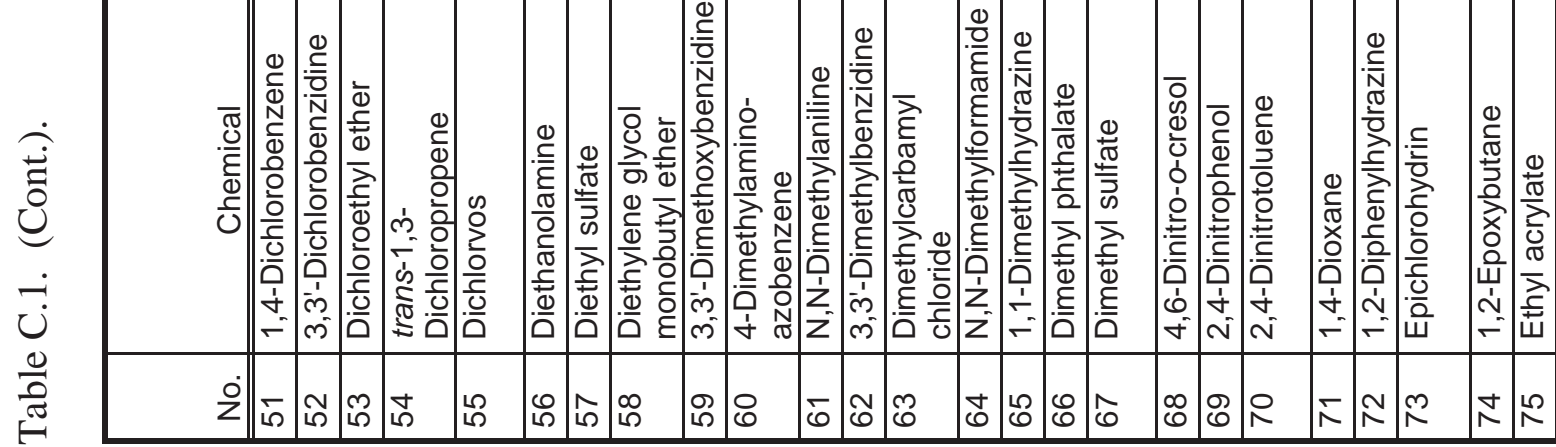




\begin{tabular}{|c|c|c|c|c|c|c|c|c|c|c|c|c|c|c|c|c|c|c|c|c|c|c|c|c|}
\hline 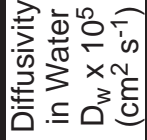 & $\mid \begin{array}{l}\mathcal{N} \\
\tilde{D} \\
0 \\
0 \\
0 \\
0\end{array}$ & 음 & & 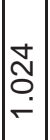 & $\left|\begin{array}{l}0 \\
\stackrel{0}{0} \\
- \\
-\end{array}\right|$ & $\mid \begin{array}{l}\stackrel{P}{2} \\
\stackrel{N}{N} \\
-\end{array}$ & 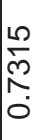 & مَ & 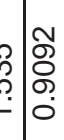 & & 年 & 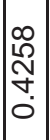 & 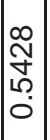 & 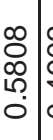 & & 令 & $\mid \begin{array}{l}0 \\
\frac{\sigma}{1} \\
0 \\
0 \\
0 \\
0\end{array}$ & $\begin{array}{l}1 \\
0 \\
0 \\
0 \\
0 \\
0 \\
0\end{array}$ & 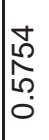 & 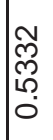 & 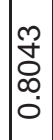 & & 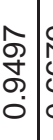 & \\
\hline 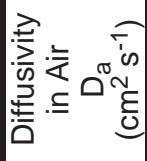 & $\mid \begin{array}{l}8 \\
0 \\
0 \\
0 \\
0 \\
0 \\
0\end{array}$ & 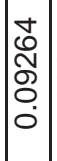 & 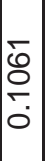 & 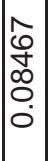 & 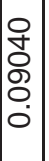 & $\frac{m}{\frac{m}{2}}$ & $\begin{array}{l}4 \\
\frac{0}{1} \\
0 \\
0 \\
0\end{array}$ & 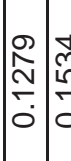 & 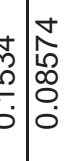 & $\begin{array}{l}\text { 웅 } \\
\text { Oे } \\
0\end{array}$ & $\begin{array}{c}\text { N } \\
\\
.\end{array}$ & 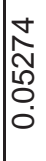 & $\left|\begin{array}{l}\hat{1} \\
0 \\
0 \\
0 \\
0 \\
0\end{array}\right|$ & \begin{tabular}{l|l}
$\overline{0}$ & \\
0 & \\
0 & \\
0 & \\
0 &
\end{tabular} & 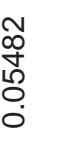 & \begin{tabular}{l}
$\approx$ \\
$\infty$ \\
\multirow{2}{0}{} \\
0 \\
0 \\
0
\end{tabular} & $\begin{array}{l}\widetilde{1} \\
\infty \\
\infty \\
0 \\
0 \\
0 \\
0 \\
0\end{array}$ & 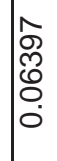 & 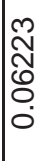 & 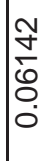 & $\begin{array}{l}0 \\
0 \\
0 \\
0 \\
0 \\
0\end{array}$ & & 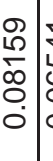 & 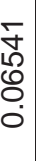 \\
\hline
\end{tabular}

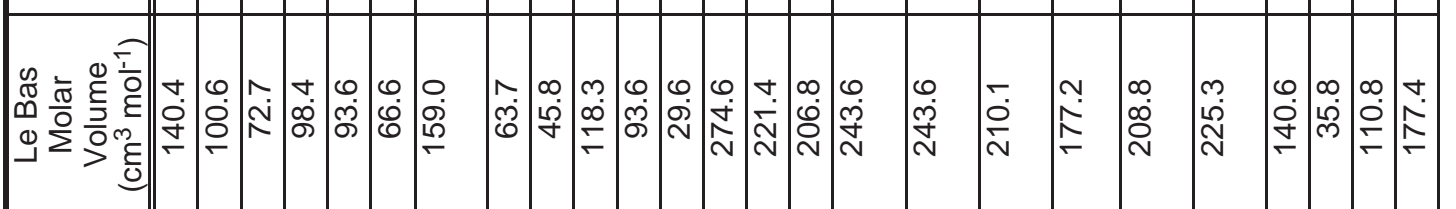

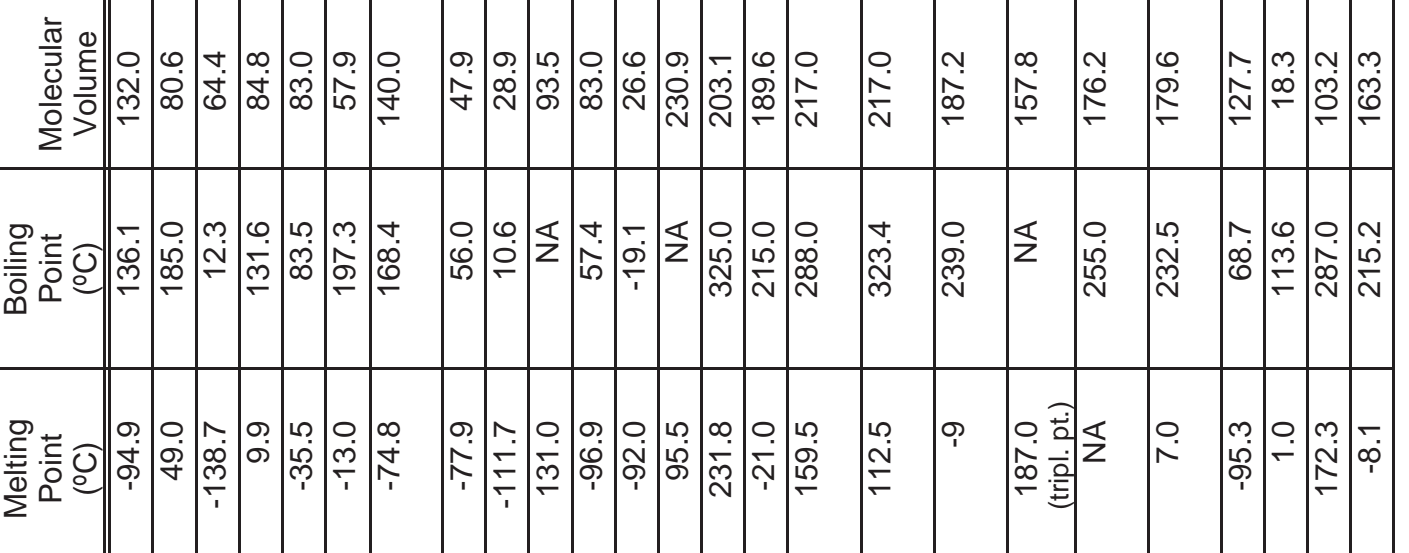

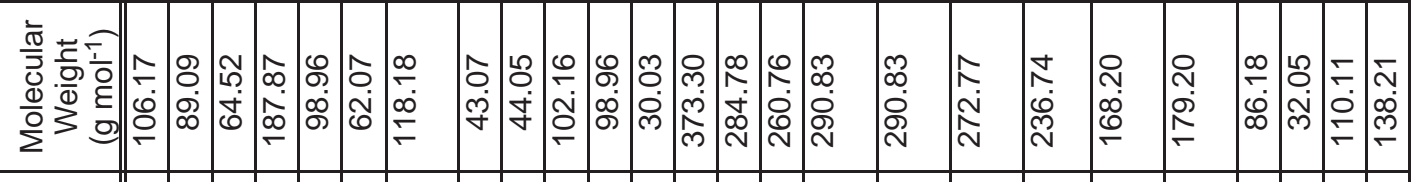

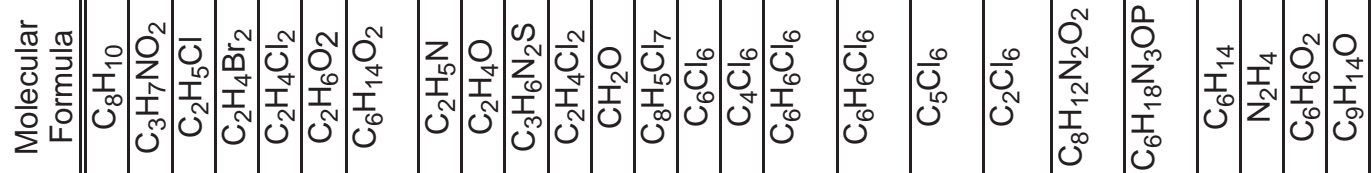

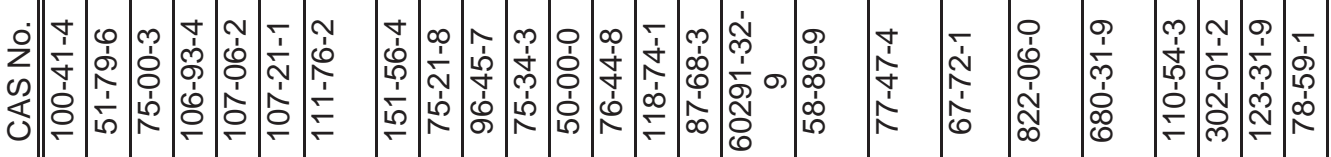

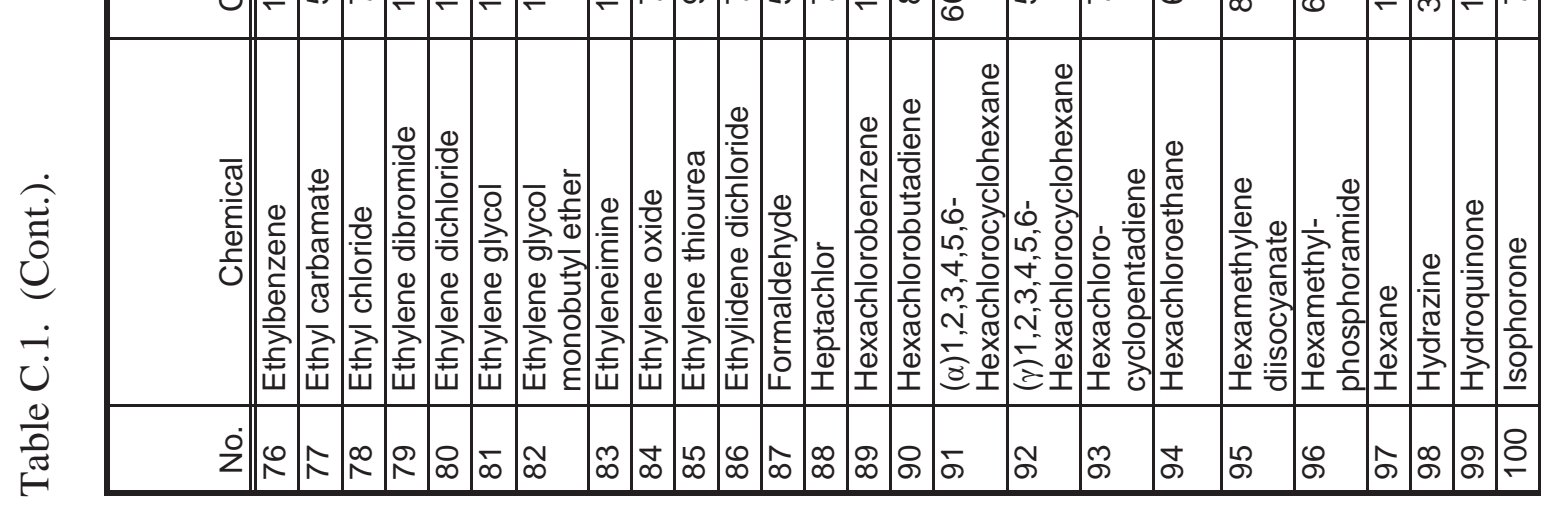




\begin{tabular}{|c|c|c|c|c|c|c|c|c|c|c|c|c|c|c|c|c|c|c|c|c|c|c|c|}
\hline 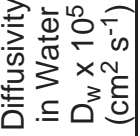 & $\frac{8}{8}$ & 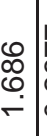 & $\begin{array}{c}\hat{N} \\
\text { o } \\
\text { o. } \\
0\end{array}$ & 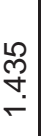 & $\begin{array}{l}\hat{n} \\
\hat{o} \\
\dot{-}\end{array}$ & 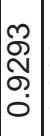 & 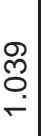 & 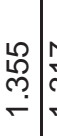 & & 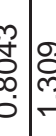 & 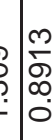 & b. & 孚 & ్ָల్ & 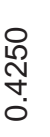 & 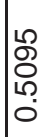 & $\mid \begin{array}{c}1 \\
2 \\
n \\
2 \\
0 \\
0\end{array}$ & & 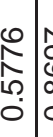 & 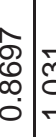 & כִ. & $\infty$ & $\mid \begin{array}{l}0 \\
\stackrel{n}{n} \\
\infty \\
\infty \\
0 \\
0\end{array}$ \\
\hline 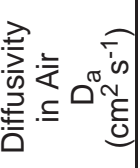 & 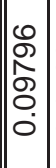 & \begin{tabular}{l|l}
$\infty$ \\
0 \\
\\
\\
\end{tabular} & 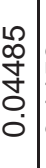 & 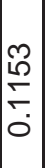 & 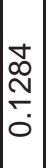 & 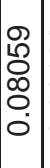 & $\begin{array}{l}8 \\
\infty \\
\\
0 \\
0 \\
0\end{array}$ & 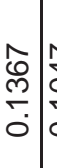 & 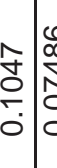 & 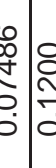 & 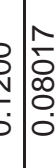 & $\mid$\begin{tabular}{l}
$m$ \\
\multirow{2}{\infty}{} \\
$\infty$ \\
0 \\
0 \\
0
\end{tabular} & $\begin{array}{l}0 \\
0 \\
18 \\
0 \\
0\end{array}$ & $\begin{array}{l}m \\
\mathscr{\delta} \\
0 \\
0 \\
0\end{array}$ & 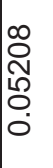 & $\begin{array}{l}\text { D } \\
0 \\
0 \\
0 \\
0 \\
0 \\
0\end{array}$ & 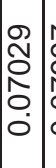 & $\begin{array}{l}\hat{2} \\
\text { ब. } \\
0 \\
0 \\
0\end{array}$ & 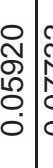 & 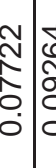 & 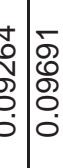 & 孚 & 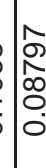 \\
\hline 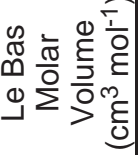 & $\stackrel{\infty}{\infty}$ & م. & $\begin{array}{l}- \\
\infty \\
\infty \\
\infty\end{array}$ & 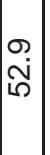 & $\left|\begin{array}{l}\mid \\
0 \\
0 \\
0\end{array}\right|$ & 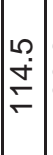 & $\begin{array}{l}\sim \\
\mathscr{\leftrightarrow} \\
\mathscr{\leftrightarrow}\end{array}$ & 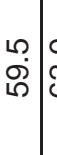 & 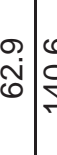 & $\begin{array}{l}0 \\
\dot{0} \\
\dot{1}\end{array}$ & $\stackrel{\infty}{\stackrel{\infty}{N}}$ & ì & 吕 & $\frac{\dot{T}}{\pi}$ & $\begin{array}{l}0 \\
\stackrel{0}{\alpha} \\
\end{array}$ & 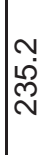 & 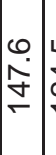 & $\begin{array}{l}\stackrel{\sim}{+} \\
\stackrel{+}{-}\end{array}$ & 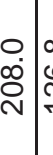 & & יִ & & : \\
\hline 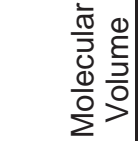 & $\left(\begin{array}{c}\infty \\
\infty \\
0 \\
0\end{array}\right.$ & m & 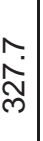 & $\begin{array}{l}N \\
\dot{g}\end{array}$ & $\begin{array}{l}\infty \\
\dot{\gamma} \\
\dot{\gamma}\end{array}$ & 술. & $\begin{array}{l}\sim \\
\infty \\
\infty \\
\infty\end{array}$ & 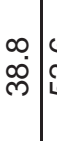 & 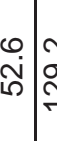 & \begin{tabular}{c|c} 
\\
Na \\
\end{tabular} & 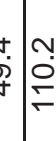 & 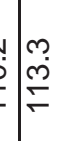 & 苘 & 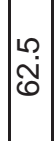 & $\begin{array}{l}\text { m} \\
\stackrel{\leftrightarrow}{+} \\
\sim\end{array}$ & $\frac{n}{i}$ & $\mid$\begin{tabular}{l}
0 \\
$\dot{q}$ \\
$\dot{1}$ \\
\hdashline \\
$\vdots$
\end{tabular} & ㄴ. & 我 & & 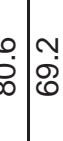 & & 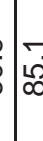 \\
\hline 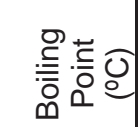 & 尺 & $\begin{array}{l}0 \\
\dot{0}\end{array}$ & & 吕 & $\mid \begin{array}{l}0 \\
\dot{\sim} \\
\underset{\sim}{\sim}\end{array}$ & $\left|\begin{array}{l}0 \\
\dot{⿰} \\
\end{array}\right|$ & $\begin{array}{l}0 \\
\stackrel{2}{N}\end{array}$ & 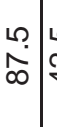 & 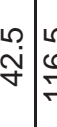 & 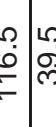 & 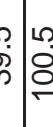 & 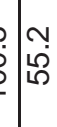 & $\mathbb{z}$ & $\begin{array}{l}0 \\
\dot{y} \\
\dot{y}\end{array}$ & $\$$ & $\mathscr{D}^{\infty}$ & 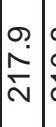 & \begin{tabular}{l|l}
$\infty$ & \\
$\dot{0}$ \\
\end{tabular} & 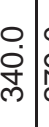 & & 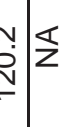 & $\begin{array}{l}0 \\
15 \\
15\end{array}$ & 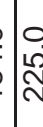 \\
\hline 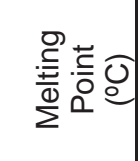 & م & $\begin{array}{l}0 \\
\text { के } \\
\text { के }\end{array}$ & $\begin{array}{l}0 \\
\stackrel{1}{\infty} \\
\infty\end{array}$ & 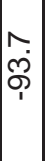 & 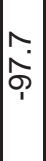 & $\begin{array}{l}+ \\
\dot{0} \\
\grave{1}\end{array}$ & $\begin{array}{l}\hat{1} \\
\dot{0} \\
0 \\
1\end{array}$ & 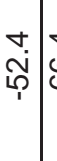 & 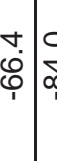 & 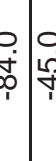 & 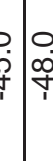 & 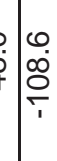 & $\mathbb{z}$ & 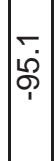 & 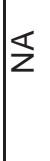 & مٌ & \begin{tabular}{|l}
$N$ \\
\hdashline \\
$\infty$ \\
$\infty$
\end{tabular} & مْ & \pm & 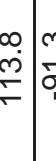 & 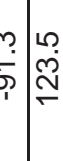 & 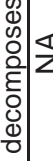 & : \\
\hline
\end{tabular}

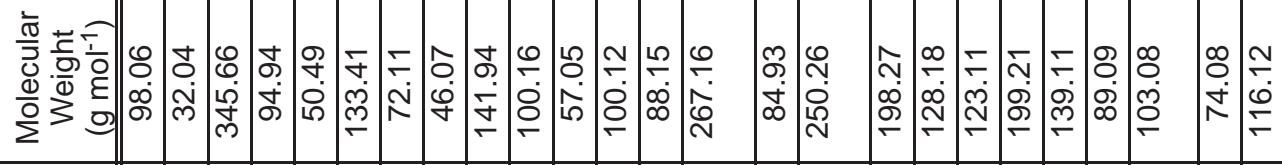

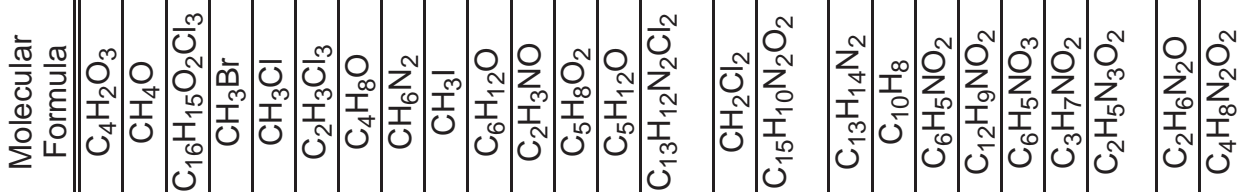

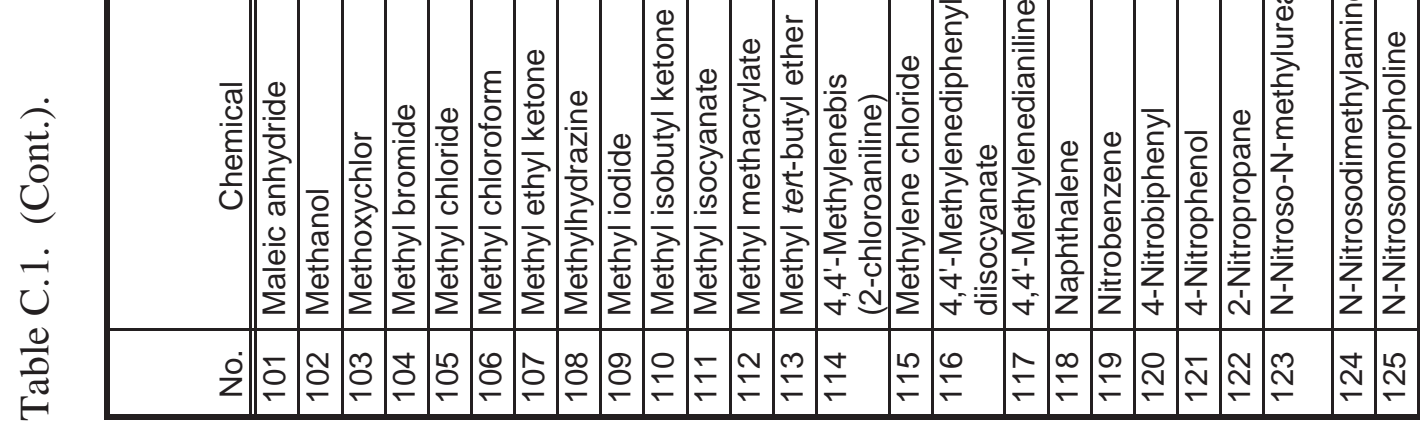




\begin{tabular}{|c|c|c|c|c|c|c|c|c|c|c|c|c|c|c|c|c|c|c|c|c|c|c|c|}
\hline 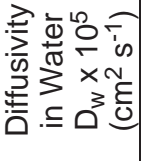 & 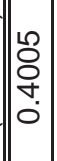 & & $\begin{array}{l}0 \\
0 \\
0 \\
0 \\
0 \\
0\end{array}$ & $\stackrel{0}{0}$ & $\mid \begin{array}{l}\infty \\
\infty \\
0 \\
\infty \\
0 \\
0\end{array}$ & & $\mid \begin{array}{l}0 \\
\frac{0}{N} \\
-\end{array}$ & $\mid$\begin{tabular}{l|l}
0 & 1 \\
0 & 1 \\
$\vdots$ & 1 \\
$\infty$ & 1 \\
0 & \\
0
\end{tabular} & $\begin{array}{l}\hat{a} \\
0 \\
\hat{0} \\
0 \\
0\end{array}$ & 芯 & 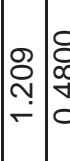 & 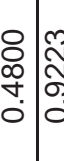 & & 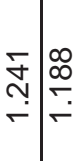 & 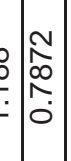 & \begin{tabular}{l|l}
$\mathscr{N}$ & $\hat{N}$ \\
$\mathcal{N}$ & \\
$\mathscr{O}$ & $\infty$ \\
0 & $\infty$ \\
0
\end{tabular} & 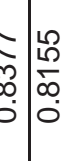 & 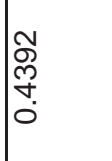 & $\begin{array}{c}\text { P } \\
\text { o } \\
0 \\
0\end{array}$ & 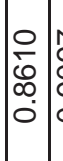 & 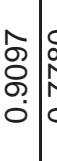 & & 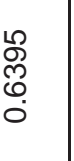 \\
\hline 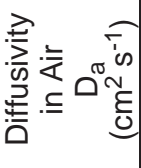 & $\mid \begin{array}{l}\hat{0} \\
8 \\
0 \\
0 \\
0 \\
0\end{array}$ & 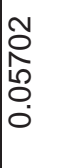 & $\mid \begin{array}{l}m \\
\mathscr{3} \\
\infty \\
0 \\
0 \\
0 \\
0\end{array}$ & $\mid \begin{array}{l} \\
\\
D \\
0 \\
0 \\
0 \\
0\end{array}$ & $\mid \begin{array}{c}\infty \\
0 \\
0 \\
0 \\
0 \\
0 \\
0\end{array}$ & $\begin{array}{l}\dot{1} \\
0 \\
0 \\
0 \\
\end{array}$ & $\begin{array}{l}0 \\
0 \\
0 \\
0 \\
0\end{array}$ & 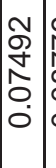 & $\begin{array}{l}0 \\
R \\
0 \\
0 \\
0 \\
0\end{array}$ & 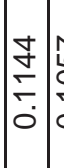 & 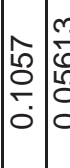 & 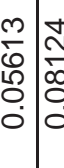 & 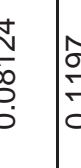 & 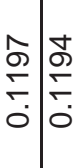 & 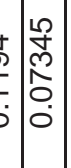 & \begin{tabular}{|l|l} 
& 8 \\
W & 0 \\
$\infty$ & 0 \\
0 & 0 \\
0 & 0 \\
0 & 0
\end{tabular} & 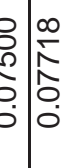 & 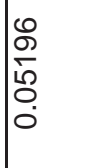 & $\begin{array}{l}\overline{0} \\
\tilde{N} \\
0 \\
0 \\
0\end{array}$ & 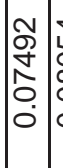 & 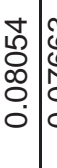 & \begin{tabular}{l|l}
0 & \\
0 & \\
0 & 0 \\
0 & 0 \\
0 & 0 \\
0 & 0 \\
0
\end{tabular} & $\begin{array}{l}\bar{c} \\
\vdots \\
0 \\
0 \\
0 \\
0\end{array}$ \\
\hline 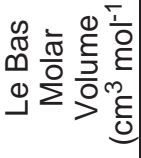 & $\mid \begin{array}{l}\infty \\
\infty \\
\infty \\
\infty \\
\sim\end{array}$ & ๙্ & Õ & ڤं & $\mid \begin{array}{l}\stackrel{+}{\dot{d}} \\
\stackrel{\sim}{\sim}\end{array}$ & $\frac{\dot{v}}{\grave{r}}$ & $\begin{array}{l}\hat{n} \\
\dot{\rho} \\
\rho\end{array}$ & 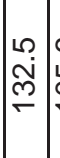 & \begin{tabular}{l}
0 \\
0 \\
0 \\
0 \\
\hdashline
\end{tabular} & $\overline{8}: 5$ & \begin{tabular}{l|l}
0 & $m$ \\
$\dot{T}$ & $\alpha$ \\
$\alpha$ & $a$ \\
\end{tabular} & 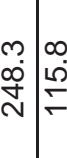 & $\begin{array}{l}0 \\
\stackrel{0}{0} \\
\end{array}$ & 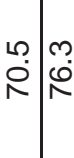 & 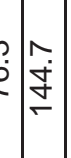 & 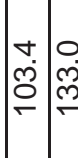 & 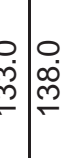 & $\begin{array}{l}\infty \\
\stackrel{\infty}{\infty} \\
\stackrel{\infty}{N}\end{array}$ & ڤ্ं & 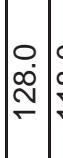 & 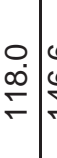 & & $\begin{array}{l}\stackrel{+}{0} \\
\infty \\
\stackrel{\infty}{ }\end{array}$ \\
\hline 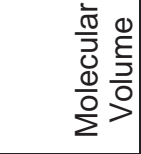 & | & ه্ & 吕 & ब̄. & $\begin{array}{l}\hat{1} \\
\dot{0} \\
\\
\end{array}$ & $\begin{array}{l}0 \\
\dot{0}\end{array}$ & $\begin{array}{l}\infty \\
\stackrel{\infty}{\infty} \\
\mathbb{N}\end{array}$ & $\mid$\begin{tabular}{c}
$\sim$ \\
$\infty$ \\
\hdashline \\
\hdashline
\end{tabular} & 足 & \begin{tabular}{l|l}
0 & 1 \\
$\dot{10}$ & 1 \\
10 & 0
\end{tabular} & $\mid \begin{array}{l}\hat{i} \\
\hat{0} \\
\hat{0}\end{array}$ & 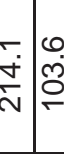 & مُ & \begin{tabular}{|l|l|l}
$\dot{\sigma}$ \\
$\dot{\sigma}$ \\
$\dot{\sigma}$
\end{tabular} & 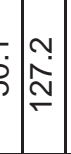 & 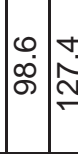 & 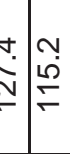 & 守 & ¿̊. & 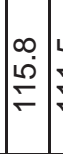 & 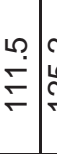 & & $\begin{array}{l}0 \\
\stackrel{8}{8} \\
\stackrel{8}{0}\end{array}$ \\
\hline 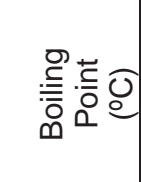 & 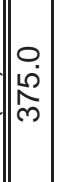 & 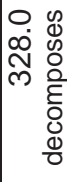 & $\begin{array}{ll}0 & 0 \\
0 & 0 \\
0 & 0 \\
\infty & 0 \\
0 & 0 \\
0 \\
0 \\
0 \\
0\end{array}$ & $\mid$ & $\begin{array}{l}0 \\
0 \\
\dot{i} \\
\stackrel{N}{ }\end{array}$ & $\begin{array}{l}0 \\
\infty \\
\infty\end{array}$ & $\mid \begin{array}{c}\infty \\
i \\
\infty \\
1\end{array}$ & $\begin{array}{l}\text { O } \\
\stackrel{\leftrightarrow}{\mathscr{N}} \\
\text { N }\end{array}$ & 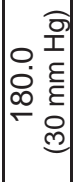 & 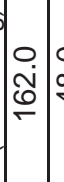 & 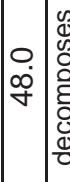 & 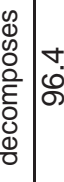 & in & 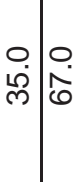 & 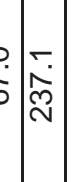 & 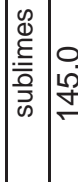 & \begin{tabular}{l|l}
0 \\
$\dot{f}$ \\
\hdashline
\end{tabular} & $\begin{array}{l}n \\
0 \\
0 \\
8 \\
8\end{array}$ & 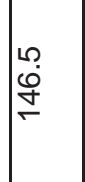 & $\left(\begin{array}{l}m \\
\dot{N} \\
\stackrel{N}{T}\end{array}\right.$ & 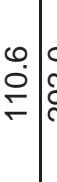 & & $\stackrel{5}{\sim}$ \\
\hline 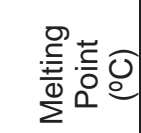 & 6 & $\begin{array}{l}0 \\
\dot{J} \\
\end{array}$ & $\begin{array}{l}0 \\
\stackrel{i}{N} \\
-\end{array}$ & 审 & \begin{tabular}{|c|}
0 \\
$\dot{6}$ \\
+1 \\
\end{tabular} & 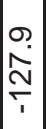 & $\begin{array}{l}0 \\
\stackrel{\mathscr{m}}{m} \\
\overline{1}\end{array}$ & $\begin{array}{l}\infty \\
0 \\
\dot{m} \\
- \\
\end{array}$ & o & 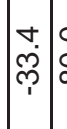 & \begin{tabular}{|c|c}
0 & 0 \\
0 & $\infty$ \\
$\infty$ & $\infty$ \\
1 & $\infty$
\end{tabular} & 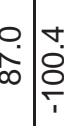 & : & 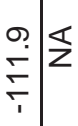 & 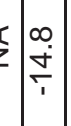 & 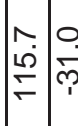 & 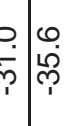 & $\begin{array}{l}0 \\
\text { L } \\
0 \\
0\end{array}$ & 䎹 & 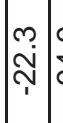 & 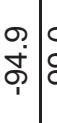 & & ¿ุ \\
\hline 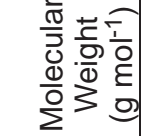 & 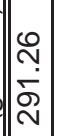 & 点 & 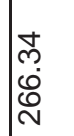 & $\begin{array}{l}\mp \\
\dot{\sigma} \\
\dot{\sigma}\end{array}$ & $\mid$\begin{tabular}{l}
$\forall$ \\
\hdashline \\
$\infty$ \\
0 \\
\end{tabular} & $\begin{array}{l}\sim \\
\tilde{\sigma} \\
\infty \\
\sigma\end{array}$ & $\begin{array}{l}8 \\
\dot{0} \\
\dot{m}\end{array}$ & $\mid$\begin{tabular}{c}
$\cong$ \\
\hdashline$\infty$ \\
$o$ \\
$\stackrel{+}{-}$
\end{tabular} & İ & 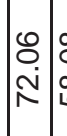 & 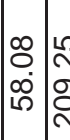 & 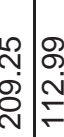 & & \begin{tabular}{l|l}
$\infty$ & 0 \\
0 & 0 \\
$\infty$ & 0 \\
$i n$ & 10 \\
$i n$
\end{tabular} & 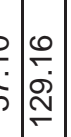 & 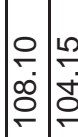 & 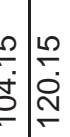 & 䑧 & $\begin{array}{l}\infty \\
\infty \\
\\
\underline{0} \\
\end{array}$ & $\left|\begin{array}{c}\mathscr{\infty} \\
\infty \\
\infty \\
\infty \\
0 \\
0\end{array}\right|$ & 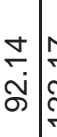 & & $\begin{array}{l}\frac{0}{N} \\
\stackrel{+}{+}\end{array}$ \\
\hline 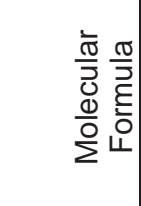 & 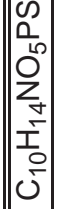 & $\begin{array}{l}O^{N} \\
\sum_{L} \\
0 \\
0 \\
0\end{array}$ & $\begin{array}{l}I \\
\text { I } \\
\frac{10}{0} \\
0 \\
0\end{array}$ & $\begin{array}{c}1 \\
0 \\
0 \\
10 \\
0 \\
0 \\
0\end{array}$ & $\mid \begin{array}{l}2 \\
\sum_{\infty}^{\infty} \\
I \\
0 \\
0\end{array}$ & & $\frac{n}{1}$ & $\mid \begin{array}{c}\infty \\
0 \\
I^{\infty} \\
0 \\
0\end{array}$ & 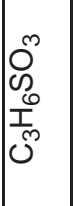 & 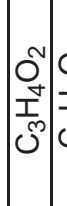 & 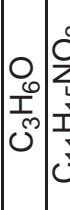 & 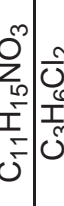 & & 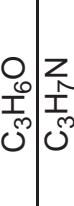 & 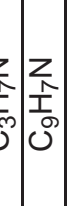 & 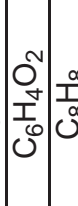 & 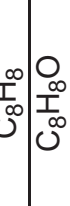 & 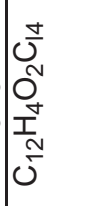 & $\begin{array}{l}\frac{N}{U} \\
\tilde{N}^{N} \\
\tilde{N}^{N}\end{array}$ & 式 & 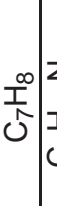 & 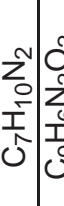 & 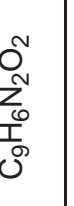 \\
\hline $\begin{array}{l}0 \\
2 \\
0 \\
0 \\
0\end{array}$ & $\mid \begin{array}{l}\infty \\
\infty \\
\infty \\
\infty \\
\vdots \\
0 \\
\omega\end{array}$ & 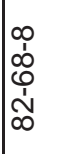 & $\mid \begin{array}{l}10 \\
0 \\
0 \\
0 \\
1 \\
1 \\
\infty\end{array}$ & 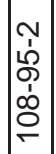 & \begin{tabular}{|l}
$\mid$ \\
0 \\
0 \\
1 \\
0 \\
0 \\
0 \\
\end{tabular} & 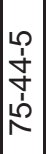 & 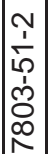 & 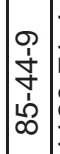 & 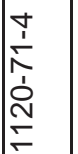 & 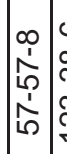 & 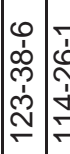 & 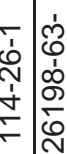 & 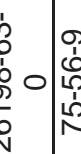 & 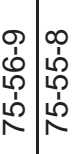 & 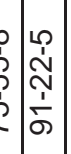 & 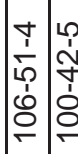 & 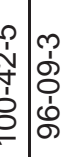 & 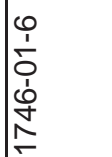 & 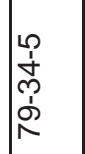 & 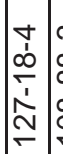 & 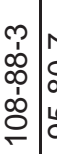 & & $\begin{array}{l}9 \\
1 \\
\infty \\
1 \\
1 \\
0 \\
0 \\
0\end{array}$ \\
\hline 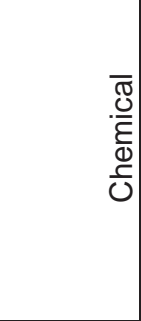 & 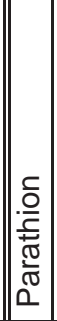 & 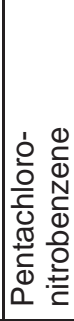 & 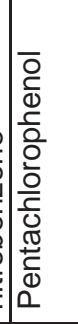 & \begin{tabular}{|c|} 
\\
$\overline{0}$ \\
$\frac{0}{0}$ \\
$\frac{\bar{c}}{\alpha}$ \\
\end{tabular} & 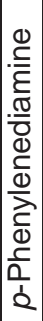 & 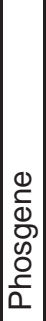 & 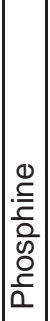 & 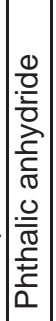 & 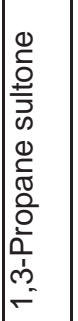 & 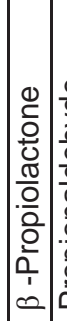 & 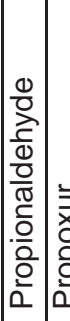 & 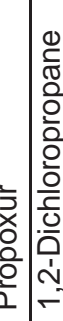 & 步 & 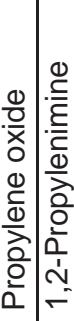 & 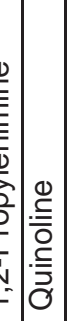 & 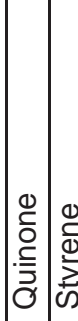 & 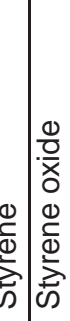 & 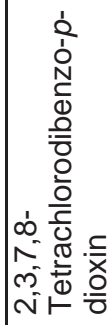 & 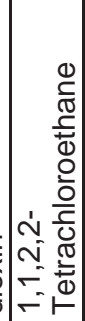 & 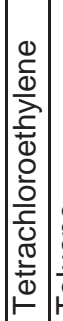 & 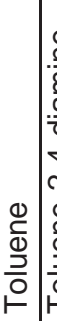 & 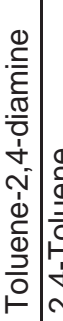 & 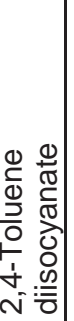 \\
\hline í & 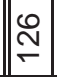 & $\stackrel{\hat{N}}{-}$ & $\stackrel{\infty}{\stackrel{\sim}{\sim}}$ & $\stackrel{\overbrace{}}{\stackrel{N}{\sim}}$ & 오 & $\bar{m}$ & $\stackrel{\widetilde{m}}{\sim}$ & $\stackrel{m}{\longrightarrow}$ & 岕 & 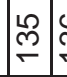 & $\begin{array}{c}\mathscr{N} \\
\stackrel{N}{\longrightarrow}\end{array}$ & $\begin{array}{l}m \\
m\end{array}$ & 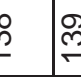 & 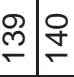 & \begin{tabular}{l|l|}
$f$ & - \\
\end{tabular} & 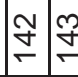 & $\begin{array}{l}\dot{P} \\
\dot{P}\end{array}$ & $\stackrel{0}{+}$ & $\stackrel{O}{\square}$ & 守 & $\stackrel{\infty}{\stackrel{\infty}{+}}$ & 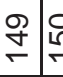 & 온 \\
\hline
\end{tabular}




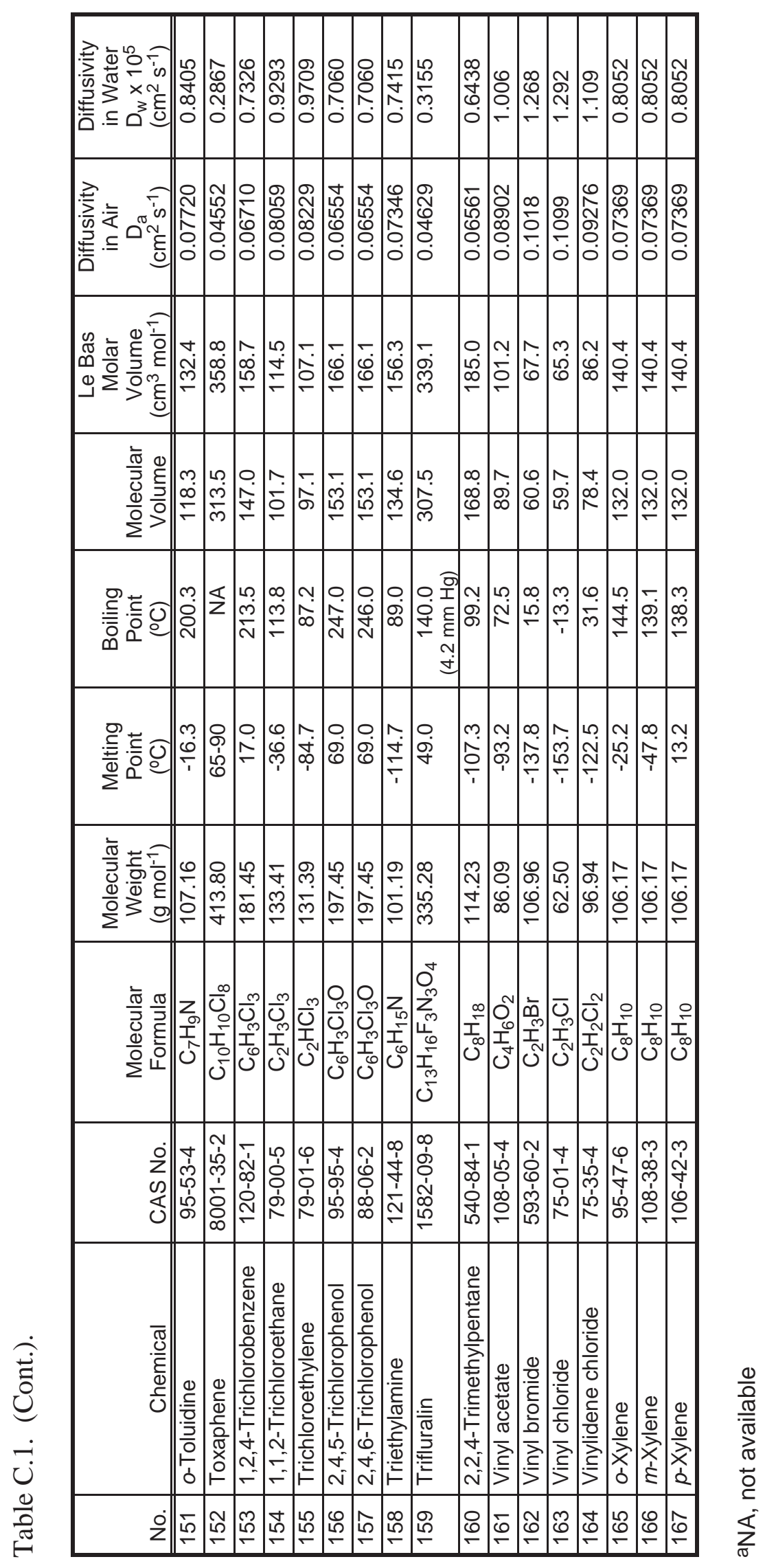




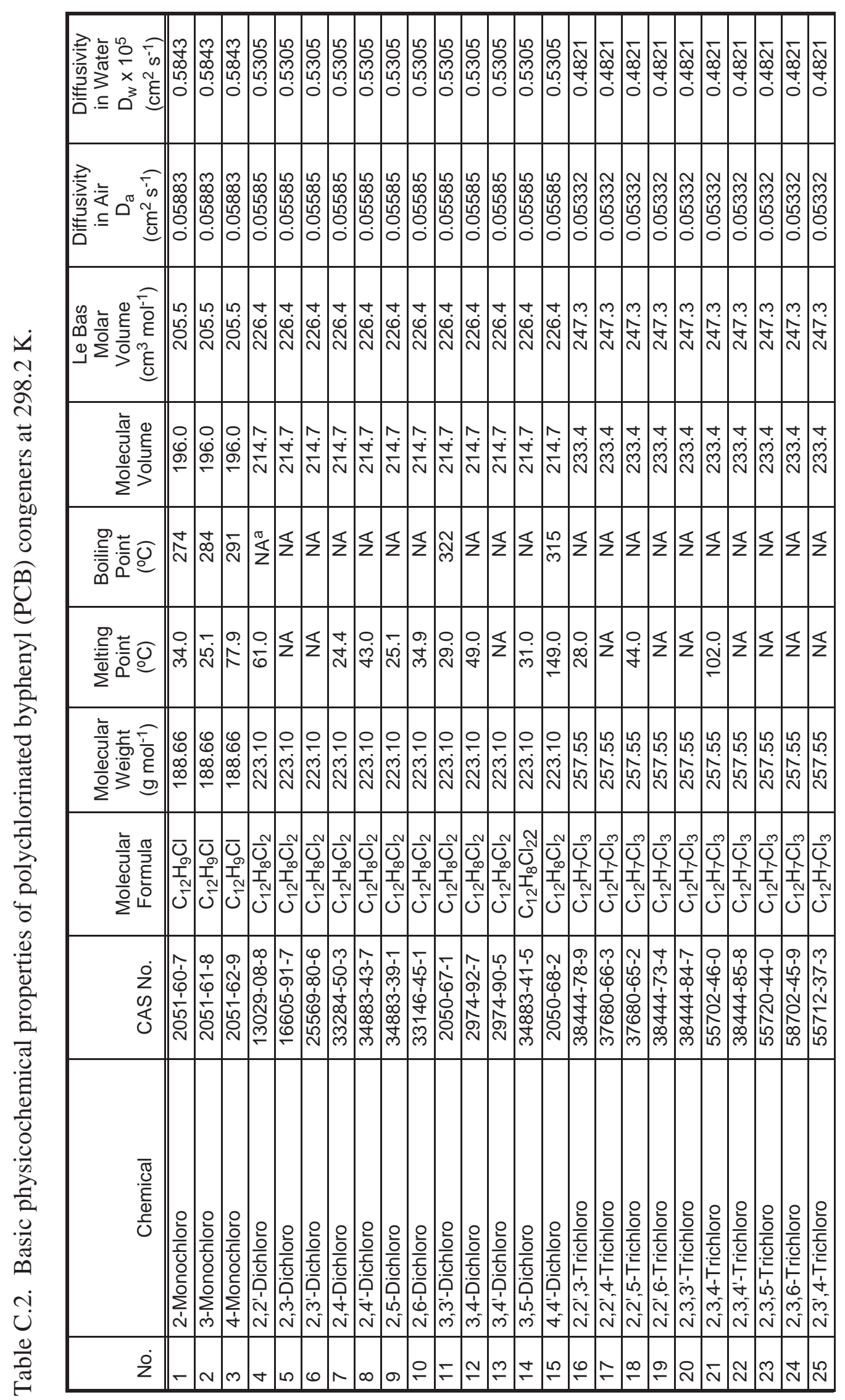




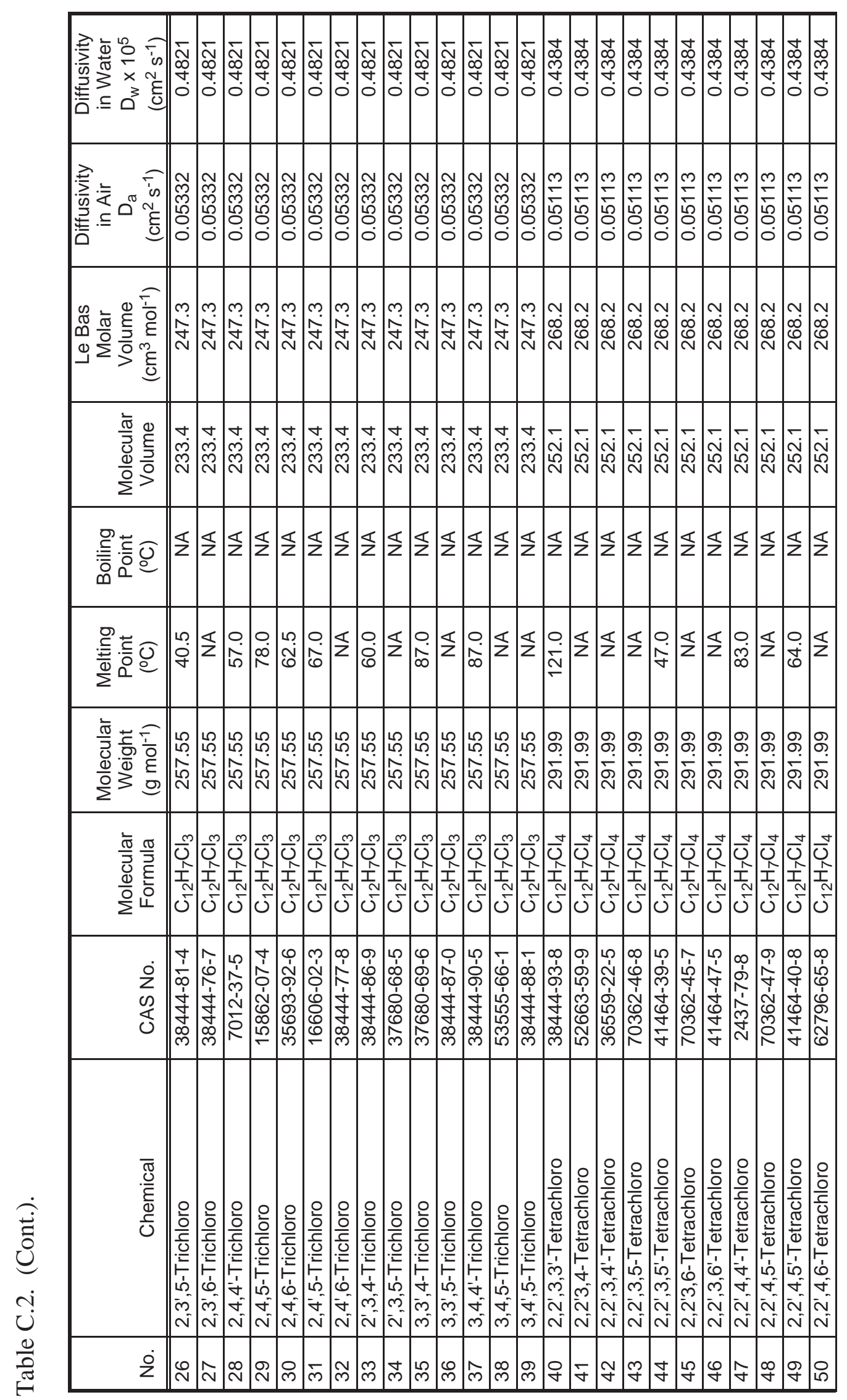




\begin{tabular}{|c|c|c|c|c|c|c|c|c|c|c|c|c|c|c|c|c|c|c|c|c|c|c|c|c|c|}
\hline 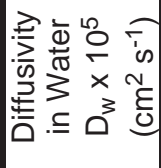 & 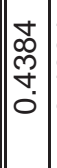 & & $\mid$\begin{tabular}{l}
+ \\
$\infty$ \\
o \\
\multirow{2}{0}{} \\
0
\end{tabular} & 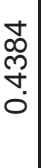 & 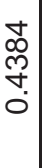 & $\begin{array}{l}+ \\
0 \\
\tilde{m} \\
\vdots \\
0\end{array}$ & $\mid$ & 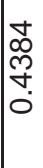 & 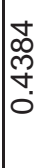 & 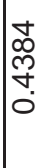 & 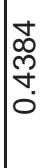 & & & 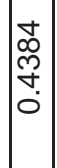 & 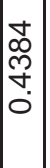 & $\begin{array}{l}+ \\
\infty \\
\text { o } \\
\vdots \\
0\end{array}$ & 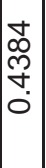 & & & & & & & & \\
\hline 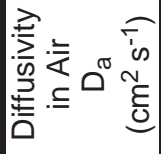 & $\mid \begin{array}{c}m \\
\frac{m}{2} \\
0 \\
0\end{array}$ & $\mid \begin{array}{l}m \\
\frac{m}{5} \\
0 \\
0 \\
0 \\
0\end{array}$ & $\begin{array}{l}m \\
\frac{m}{2} \\
0 \\
0 \\
0 \\
0\end{array}$ & $\begin{array}{c}\frac{m}{2} \\
\frac{1}{8} \\
0 \\
0\end{array}$ & $\begin{array}{l}m \\
\frac{m}{2} \\
0 \\
0 \\
0\end{array}$ & $\begin{array}{l}m \\
\frac{m}{2} \\
\\
0 \\
0\end{array}$ & $\begin{array}{l}\frac{m}{2} \\
\frac{1}{2} \\
0 \\
0 \\
0\end{array}$ & $\begin{array}{l}\frac{m}{2} \\
\frac{7}{10} \\
0 \\
0 \\
0\end{array}$ & $\begin{array}{l}m \\
\frac{m}{2} \\
\frac{2}{2} \\
0 \\
0\end{array}$ & $\begin{array}{l}m \\
\frac{m}{2} \\
\frac{0}{0} \\
0 \\
0\end{array}$ & $\begin{array}{l}m \\
\frac{m}{2} \\
\frac{1}{0} \\
0 \\
0\end{array}$ & $\begin{array}{l}\frac{m}{2} \\
\frac{m}{2} \\
0 \\
0\end{array}$ & $\begin{array}{l}\frac{m}{2} \\
\frac{2}{2} \\
0 \\
0 \\
0\end{array}$ & $\begin{array}{l}\frac{m}{2} \\
\frac{1}{20} \\
0 \\
0\end{array}$ & \begin{tabular}{|l|}
$m$ \\
$\frac{m}{2}$ \\
0 \\
0 \\
0 \\
0
\end{tabular} & $\begin{array}{l}m \\
\frac{m}{5} \\
\frac{1}{0} \\
0 \\
0\end{array}$ & $\mid \begin{array}{l}m \\
\frac{m}{2} \\
\\
0 \\
0 \\
0\end{array}$ & $\mid \begin{array}{l}m \\
\frac{m}{2} \\
0 \\
0 \\
0 \\
0\end{array}$ & $\begin{array}{c}m \\
\frac{m}{2} \\
\frac{1}{2} \\
0 \\
0\end{array}$ & $\begin{array}{l}\frac{m}{2} \\
\frac{2}{2} \\
0 \\
0\end{array}$ & $\begin{array}{l}m \\
\frac{1}{2} \\
\vdots 0 \\
0 \\
0\end{array}$ & $\begin{array}{c}\frac{m}{2} \\
\frac{2}{8} \\
0 \\
0\end{array}$ & $\begin{array}{l}\frac{m}{2} \\
\frac{m}{2} \\
0 \\
0\end{array}$ & & $\begin{array}{l}m \\
\frac{m}{2} \\
\frac{1}{0} \\
0 \\
0\end{array}$ \\
\hline 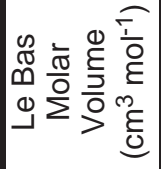 & $\mid \begin{array}{c}0 \\
N \\
o \\
o \\
N\end{array}$ & & o & & $\begin{array}{l}\sim \\
o \mathfrak{z} \\
o \\
\sim\end{array}$ & $\begin{array}{l}\sim ָ \\
o \\
o \\
\sim \\
N\end{array}$ & 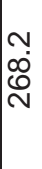 & $\begin{array}{l}\sim \\
\infty \\
\infty \\
\infty \\
\sim\end{array}$ & 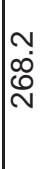 & 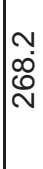 & 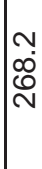 & 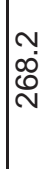 & & $\begin{array}{l}\text { va } \\
o \infty \\
o ̂ \\
N\end{array}$ & $\begin{array}{l}\sim \\
\infty \\
\infty \\
\stackrel{o}{N}\end{array}$ & $\begin{array}{l}\sim ָ \\
o \\
o \\
\sim \\
N\end{array}$ & $\mid \begin{array}{c}\sim \\
0 \\
0 \\
o \\
N\end{array}$ & 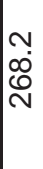 & & & & & & & 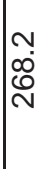 \\
\hline 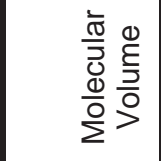 & $\begin{array}{l}- \\
\text { నิ่ } \\
\text { N }\end{array}$ & 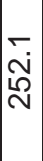 & సิ่ & స్ & $\begin{array}{c}\overline{\mathrm{N}} \\
\stackrel{\mathrm{N}}{\mathrm{N}}\end{array}$ & 灾 & స్. & స్తి & - & స్ & సิ่ & స్ & స్ & 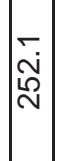 & 㲾 & స్. & 灾 & 芯 & 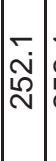 & & 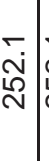 & స్. & స్. & & స్ \\
\hline 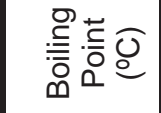 & $\mathbb{z}$ & $\$$ & \& & $\mathbb{z}$ & $\mathbb{z}$ & $\Sigma$ & $\$$ & 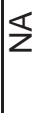 & 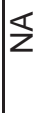 & 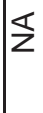 & 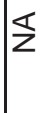 & 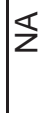 & $\S$ & $\mathbb{z}$ & $\varangle$ & 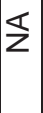 & 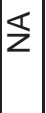 & 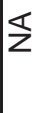 & $\mathbb{z}$ & $\Sigma$ & $\Sigma$ & $\Sigma$ & 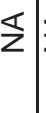 & & 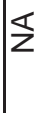 \\
\hline 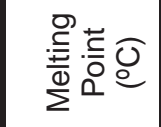 & $\varangle$ & 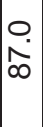 & $\begin{array}{l}0 \\
\dot{0} \\
\underline{0}\end{array}$ & $\begin{array}{l}0 \\
\infty \\
\infty \\
0 \\
\end{array}$ & 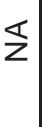 & $\Sigma$ & $\$$ & $\$$ & $\S$ & 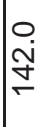 & $\begin{array}{l}\text { 오 } \\
\text { ๙ે }\end{array}$ & 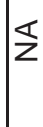 & 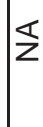 & $\S$ & \begin{tabular}{l}
0 \\
\hdashline \\
$⿱ 亠$ \\
\end{tabular} & 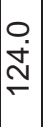 & $\Sigma$ & $\$$ & 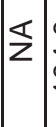 & $\begin{array}{l}0 \\
\dot{\forall} \\
\stackrel{0}{-1}\end{array}$ & $\Sigma$ & $\Sigma$ & 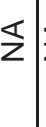 & & $\Sigma$ \\
\hline 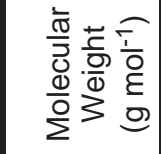 & 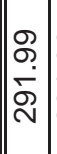 & 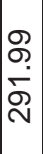 & 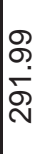 & & & 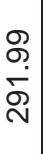 & 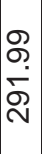 & 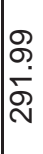 & & | & 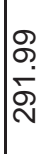 & 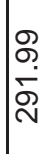 & & হ. & & & $\mid \begin{array}{l}\mathscr{S} \\
\stackrel{2}{\mathrm{~N}} \\
\end{array}$ & & & & & & & & \\
\hline 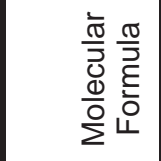 & $\mid \begin{array}{l}0 \\
0 \\
\\
N \\
0 \\
0\end{array}$ & $\mid \begin{array}{l}0 \\
0 \\
N \\
N \\
N \\
0 \\
N\end{array}$ & $\begin{array}{l}0 \\
0 \\
N^{2} \\
N^{N} \\
0\end{array}$ & 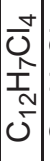 & $\begin{array}{l}0 \\
0 \\
N_{1} \\
N \\
0\end{array}$ & $\begin{array}{l}0 \\
0 \\
N_{1} \\
N \\
0\end{array}$ & $\begin{array}{l}0 \\
0 \\
1 \\
N \\
0 \\
0\end{array}$ & $\begin{array}{l}0 \\
0 \\
N^{2} \\
\text { N } \\
0\end{array}$ & 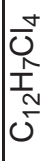 & $\begin{array}{l}0 \\
0 \\
\hat{N} \\
\text { N } \\
0\end{array}$ & 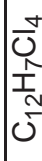 & $\mid \begin{array}{l}1 \\
0 \\
\frac{1}{1} \\
0 \\
0\end{array}$ & $\begin{array}{l}\frac{1}{0} \\
0 \\
\frac{N}{n} \\
0 \\
0\end{array}$ & $\mid \begin{array}{l}0 \\
0 \\
N \\
N \\
v \\
0 \\
N\end{array}$ & 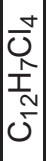 & $\begin{array}{l}0 \\
0 \\
N \\
N \\
0 \\
0\end{array}$ & 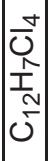 & $\mid \begin{array}{l}0 \\
0 \\
N \\
I \\
N \\
0\end{array}$ & $\mid \begin{array}{c}0 \\
0 \\
1 \\
N \\
0 \\
0\end{array}$ & $\begin{array}{l}0 \\
0 \\
\\
N \\
0\end{array}$ & $\begin{array}{l}\bar{U} \\
\bar{N} \\
\bar{N} \\
\bar{N}\end{array}$ & 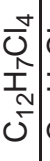 & $\begin{array}{l}\bar{N} \\
N^{N} \\
\vdots \\
0\end{array}$ & & 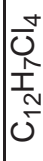 \\
\hline $\begin{array}{l}2 \\
2 \\
0 \\
0 \\
0\end{array}$ & $\mid \begin{array}{c}1 \\
\dot{1} \\
0 \\
\dot{j} \\
0 \\
1 \\
0 \\
0\end{array}$ & 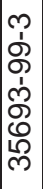 & 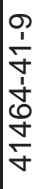 & 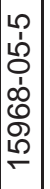 & 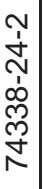 & 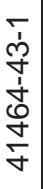 & 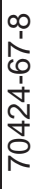 & 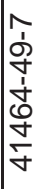 & 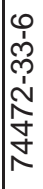 & 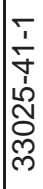 & 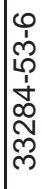 & 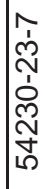 & 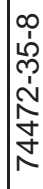 & $\begin{array}{l}0 \\
0 \\
0 \\
0 \\
0 \\
0 \\
0 \\
\mathbb{N} \\
\tilde{N}\end{array}$ & $\begin{array}{l}1 \\
\dot{1} \\
\dot{0} \\
\dot{1} \\
\infty \\
\tilde{N} \\
\tilde{m}\end{array}$ & 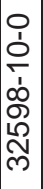 & 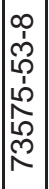 & 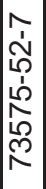 & 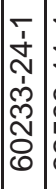 & 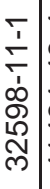 & 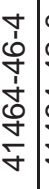 & 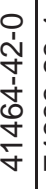 & 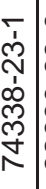 & & 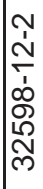 \\
\hline 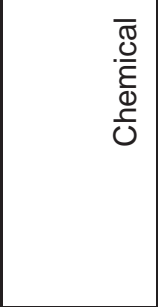 & 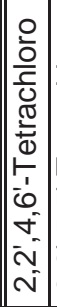 & 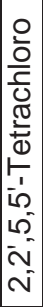 & 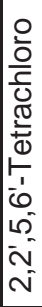 & 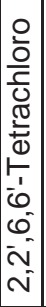 & 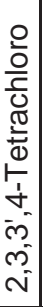 & 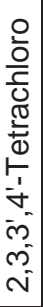 & 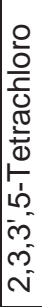 & 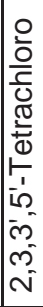 & 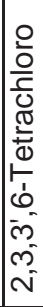 & 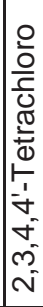 & 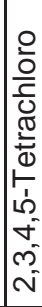 & 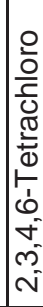 & 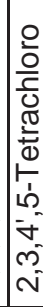 & 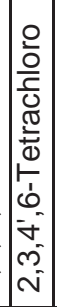 & 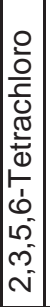 & 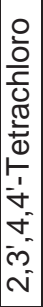 & 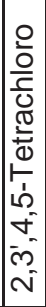 & 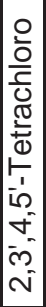 & 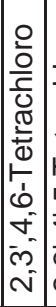 & 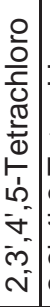 & 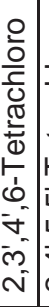 & 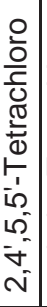 & 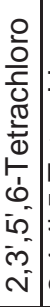 & & 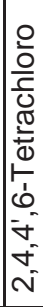 \\
\hline & is & I & I & & & & Th & |나 & & & $\overline{6}$ & 6 & & षे & 10 & 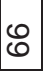 & $\hat{\omega}$ & 0 & 8 & 1 & $\bar{N}$ & $\mathbb{N}$ & $\stackrel{\Re}{\sim}$ & & \\
\hline
\end{tabular}




\begin{tabular}{|c|c|c|c|c|c|c|c|c|c|c|c|c|c|c|c|c|c|c|c|c|c|c|c|c|c|}
\hline 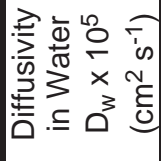 & 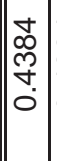 & & $\mid$\begin{tabular}{c}
\multirow{2}{*}{} \\
m \\
\multirow{\sigma}{*}{} \\
0
\end{tabular} & 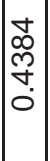 & & $\begin{array}{l}+ \\
0 \\
\tilde{m} \\
\vdots \\
0\end{array}$ & & & & م & $\mid$ & & & & & & & & & & & & & & \\
\hline 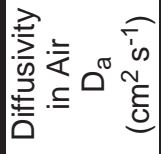 & $\mid \begin{array}{c}m \\
\frac{m}{2} \\
0 \\
0\end{array}$ & $\mid \begin{array}{l}m \\
\frac{1}{2} \\
10 \\
0 \\
0 \\
0\end{array}$ & $\mid \begin{array}{l}m \\
\frac{m}{20} \\
0 \\
0 \\
0\end{array}$ & $\begin{array}{l}\frac{m}{2} \\
\frac{1}{0} \\
0 \\
0 \\
0\end{array}$ & $\begin{array}{l}m \\
\frac{m}{2} \\
0 \\
0 \\
0\end{array}$ & $\begin{array}{l}m \\
\frac{m}{2} \\
\\
0 \\
0\end{array}$ & 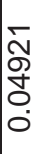 & \begin{tabular}{l}
$\bar{N}$ \\
Oे \\
\multirow{O}{0}{} \\
0 \\
0
\end{tabular} & $\begin{array}{l}\bar{N} \\
\text { Oे } \\
\text { Oे } \\
0 \\
0\end{array}$ & స్̃ & $\begin{array}{c}\overline{\grave{N}} \\
\text { Oे } \\
\\
0\end{array}$ & 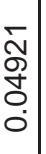 & 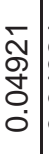 & \begin{tabular}{l}
$\bar{N}$ \\
\multirow{2}{\delta}{} \\
0 \\
$\dot{0}$
\end{tabular} & $\begin{array}{l}\bar{N} \\
\bar{\sigma} \\
\vdots \\
0 \\
0\end{array}$ & $\begin{array}{l}\bar{N} \\
\bar{o} \\
0 \\
0 \\
0\end{array}$ & $\mid \begin{array}{l}\bar{N} \\
\delta \\
0 \\
0 \\
0 \\
0\end{array}$ & 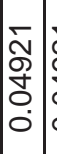 & & 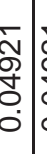 & 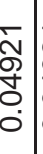 & 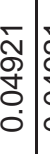 & 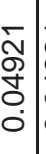 & & $\begin{array}{l}\bar{N} \\
\text { के } \\
\text { Oे } \\
0\end{array}$ \\
\hline 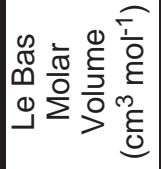 & $\mid \begin{array}{c}0 \\
N \\
o \\
o \\
N\end{array}$ & & $\begin{array}{l}N \\
\infty \\
0 \\
0 \\
N\end{array}$ & $\begin{array}{l}N \\
\infty \\
\infty \\
\infty \\
N\end{array}$ & & $\begin{array}{l}N \\
o \\
o \\
o \\
N\end{array}$ & - & - & ז্் & 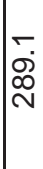 & - & $\stackrel{-}{\stackrel{\check{d}}{\mathrm{~N}}}$ & 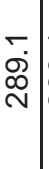 & & 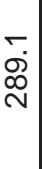 & 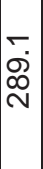 & - & & & & & 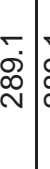 & & & 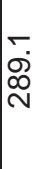 \\
\hline 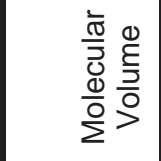 & $\begin{array}{l}- \\
\text { Nิ่ } \\
\text { Nิ }\end{array}$ & $\begin{array}{l}- \\
\text { Nิ่ } \\
\text { Nิ }\end{array}$ & సิ & $\begin{array}{l}- \\
\text { ָें } \\
\text { N̦ }\end{array}$ & $\begin{array}{c}\overline{\mathrm{N}} \\
\stackrel{\mathrm{N}}{\mathrm{N}}\end{array}$ & స్. & 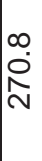 & 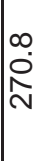 & $\begin{array}{l}\infty \\
0 \\
i \\
\end{array}$ & 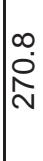 & $\mid \begin{array}{l}\infty \\
0 \\
0 \\
\\
\end{array}$ & 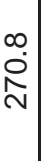 & $\begin{array}{l}\infty \\
\dot{0} \\
\text { N }\end{array}$ & $\begin{array}{l}\infty \\
\stackrel{\infty}{0} \\
N \\
N\end{array}$ & 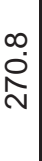 & 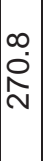 & $\begin{array}{l}\infty \\
0 \\
0 \\
N \\
N\end{array}$ & 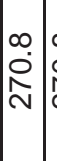 & 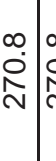 & 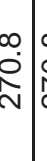 & 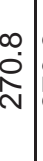 & 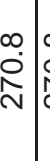 & & & م \\
\hline 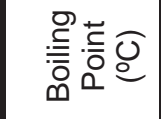 & $\mathbb{z}$ & $\mathbb{z}$ & $\Sigma$ & $\Sigma$ & $\mathbb{z}$ & $\Sigma$ & $\$$ & 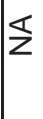 & 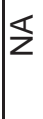 & 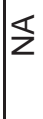 & 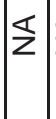 & 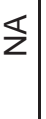 & $\S$ & $\mathbb{z}$ & $\S$ & 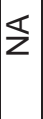 & $\Sigma$ & 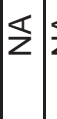 & $\Sigma \leq$ & $\mathbb{z}$ & 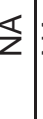 & $\mathbb{Z}$ & 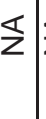 & & ¿ \\
\hline 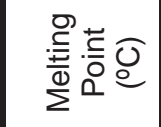 & $\varangle$ & $\begin{array}{l}0 \\
0 \\
\infty \\
0\end{array} \mid$ & $\$$ & $\Sigma$ & $\begin{array}{l}0 \\
\dot{+} \\
\stackrel{0}{0}\end{array}$ & $\mathbb{z}$ & $\$$ & $\$$ & $\$$ & $\varangle$ & 웅 & $\begin{array}{l}0 \\
\dot{+} \\
\leftarrow\end{array}$ & $\begin{array}{l}0 \\
\dot{0} \\
0 \\
\end{array}$ & $\varangle$ & $\Sigma$ & 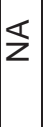 & $\Sigma$ & 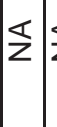 & & : & $\Sigma$ & $\Sigma$ & 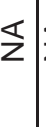 & & 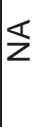 \\
\hline 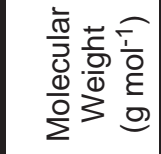 & 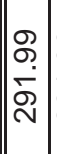 & $\mid$ & 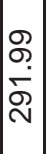 & 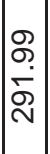 & & 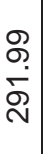 & 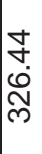 & 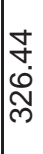 & 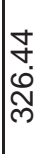 & 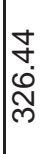 & $\mid \begin{array}{l}\mathbb{J} \\
\dot{N} \\
\tilde{m}\end{array}$ & $\mid$ & $\mid$ & 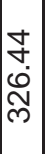 & 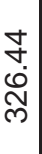 & $\begin{array}{l}\dot{Z} \\
\dot{0} \\
\dot{N} \\
\tilde{c}\end{array}$ & 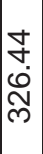 & $\left|\begin{array}{l}7 \\
\vdots \\
\dot{N} \\
m \\
m\end{array}\right|$ & 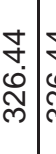 & $\begin{array}{l}8 \\
c \\
0 \\
c \\
c\end{array}$ & 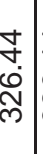 & 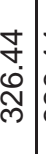 & 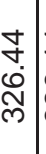 & & $\begin{array}{l}\text { Vे } \\
\stackrel{\leftrightarrow}{0} \\
\text { N }\end{array}$ \\
\hline 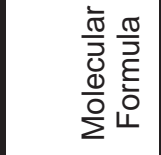 & $\mid \begin{array}{l}0 \\
0 \\
\\
N \\
0 \\
0\end{array}$ & & 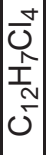 & $\mid \begin{array}{c}0 \\
0 \\
N \\
N \\
\tilde{N} \\
0\end{array}$ & $\begin{array}{l}0 \\
0 \\
N_{1} \\
N \\
0\end{array}$ & $\begin{array}{l}0 \\
0 \\
N_{1} \\
N \\
0\end{array}$ & $\begin{array}{l}\frac{10}{0} \\
10 \\
1 \\
0 \\
0\end{array}$ & $\mid \begin{array}{l}\frac{10}{0} \\
0 \\
10 \\
\frac{10}{N} \\
0 \\
0\end{array}$ & $\begin{array}{l}\frac{10}{0} \\
0 \\
10 \\
T \\
0 \\
0\end{array}$ & 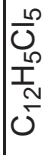 & 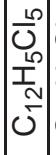 & $\mid \begin{array}{c}\frac{\omega}{0} \\
0 \\
\frac{1}{1} \\
N \\
0 \\
0\end{array}$ & $\left|\begin{array}{c}\frac{1}{0} \\
0 \\
T \\
N \\
0 \\
0\end{array}\right|$ & $\left|\begin{array}{c}\frac{1}{0} \\
0 \\
T \\
N \\
0 \\
0\end{array}\right|$ & $\begin{array}{l}\frac{L}{0} \\
\text { L } \\
T^{N} \\
0 \\
0\end{array}$ & 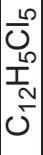 & $\mid \begin{array}{l}\frac{1}{0} \\
0 \\
1 \\
\\
0 \\
0\end{array}$ & 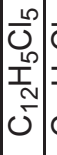 & 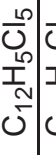 & 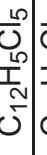 & 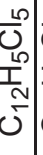 & 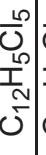 & $\begin{array}{l}\frac{10}{0} \\
\frac{10}{1} \\
j \\
0\end{array}$ & & $\begin{array}{l}\frac{10}{0} \\
0 \\
10 \\
\frac{10}{N} \\
0\end{array}$ \\
\hline 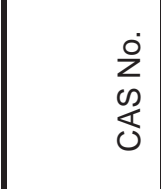 & $\mid \begin{array}{l}0 \\
0 \\
0 \\
0 \\
\vdots \\
0 \\
0 \\
0 \\
0 \\
1\end{array}$ & 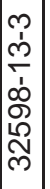 & 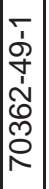 & 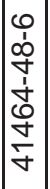 & 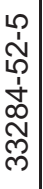 & 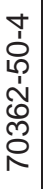 & 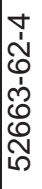 & 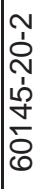 & 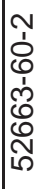 & 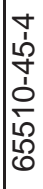 & 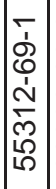 & 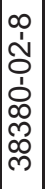 & 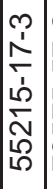 & $\begin{array}{l}N \\
1 \\
1 \\
1 \\
1 \\
N \\
N \\
N \\
N\end{array}$ & 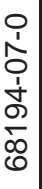 & $\begin{array}{l}\infty \\
0 \\
1 \\
0 \\
0 \\
1 \\
0 \\
0 \\
0 \\
1 \\
1\end{array}$ & 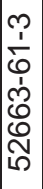 & 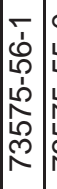 & 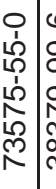 & 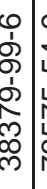 & 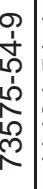 & 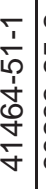 & 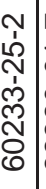 & & 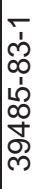 \\
\hline $\begin{array}{l}\frac{\pi}{\frac{0}{E}} \\
\frac{0}{0} \\
\frac{d}{0}\end{array}$ & 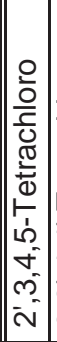 & 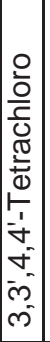 & 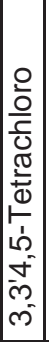 & 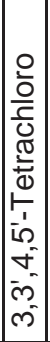 & 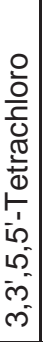 & 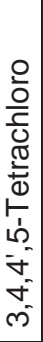 & 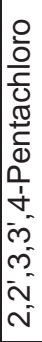 & 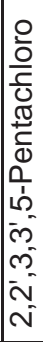 & 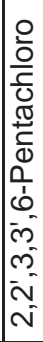 & 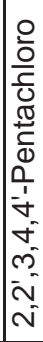 & 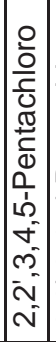 & 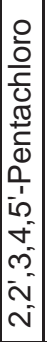 & 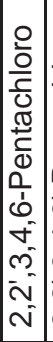 & 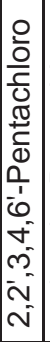 & 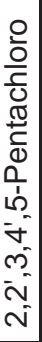 & 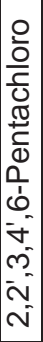 & 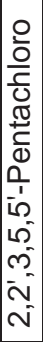 & 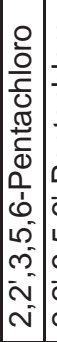 & 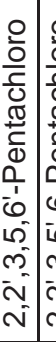 & 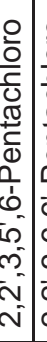 & 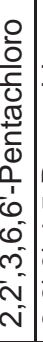 & 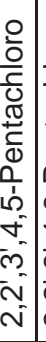 & 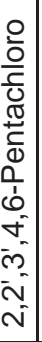 & & 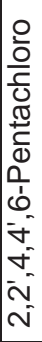 \\
\hline & $c$ & & & & & & & & & & 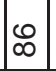 & 领 & $\ddot{\alpha}$ & & ৪ & $\sigma$ & Nু & প্. & 0 & "ै & ๘ & à & ஜ & & \\
\hline
\end{tabular}




\begin{tabular}{|c|c|c|c|c|c|c|c|c|c|c|c|c|c|c|c|c|c|c|c|c|c|c|c|c|}
\hline 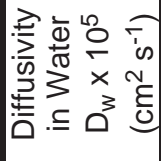 & $\mid \begin{array}{l}\infty \\
\infty \\
0 \\
0 \\
0\end{array}$ & & & & & $\begin{array}{l}0 \\
\infty \\
\infty \\
\infty \\
0 \\
0\end{array}$ & & & & 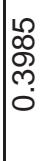 & $\mid$ & & & & & & & & 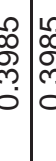 & & $b_{0}^{\infty}$ & & & \\
\hline 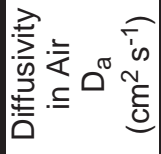 & $\mid \begin{array}{c}\bar{N} \\
\text { Oे } \\
\\
0 \\
0\end{array}$ & $\begin{array}{l}\bar{N} \\
\bar{\sigma} \\
\vdots \\
0 \\
0\end{array}$ & $\begin{array}{l}\bar{N} \\
\bar{\sigma} \\
\vdots \\
0 \\
0\end{array}$ & $\begin{array}{c}\bar{N} \\
\bar{\sigma} \\
\vdots \\
0 \\
0\end{array}$ & $\begin{array}{l}\bar{N} \\
\bar{\sigma} \\
\vdots \\
\vdots \\
0\end{array}$ & $\begin{array}{l}\bar{N} \\
\bar{\sigma} \\
0 \\
0 \\
0\end{array}$ & \begin{tabular}{l}
$\bar{N}$ \\
\multirow{O}{O}{} \\
0 \\
0 \\
0
\end{tabular} & \begin{tabular}{l}
$\bar{N}$ \\
Oे \\
\multirow{O}{0}{} \\
0 \\
0
\end{tabular} & 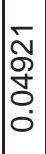 & 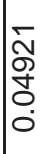 & $\begin{array}{c}\overline{\grave{N}} \\
\text { Oे } \\
\\
0\end{array}$ & 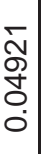 & 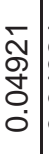 & 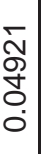 & $\begin{array}{l}\bar{N} \\
\bar{\sigma} \\
\text { Oे } \\
\dot{0}\end{array}$ & & & & 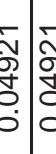 & & & & & స్̃ \\
\hline 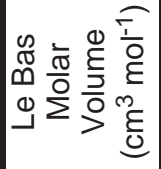 & - & 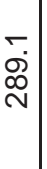 & 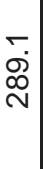 & 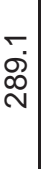 & 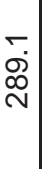 & 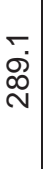 & - & - & - & - & - & 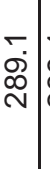 & 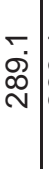 & & 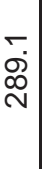 & & $\begin{array}{c}- \\
\stackrel{D}{\sim}\end{array}$ & & 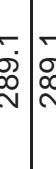 & & $\vec{b} \vec{b}$ & & & \\
\hline 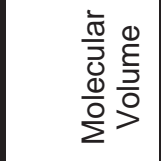 & $\mid \begin{array}{l}\infty \\
0 \\
0 \\
N\end{array}$ & 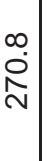 & 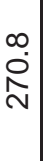 & 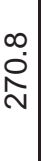 & 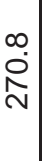 & 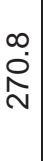 & 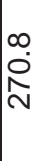 & 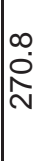 & $\mid \begin{array}{l}\infty \\
0 \\
i \\
N \\
\end{array}$ & $\mid \begin{array}{l}\infty \\
0 \\
i \\
N \\
\end{array}$ & $\mid \begin{array}{l}\infty \\
0 \\
0 \\
\\
\end{array}$ & 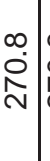 & $\begin{array}{l}\infty \\
\dot{0} \\
\text { N }\end{array}$ & 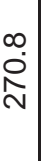 & 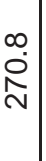 & 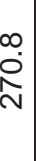 & \begin{tabular}{l|l}
$\infty$ \\
$\vdots$ \\
\\
\end{tabular} & & 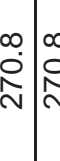 & & ja & & & \\
\hline 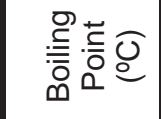 & $\mathbb{z}$ & $\mathbb{Z}$ & $\mathbb{Z}$ & $\mathbb{z}$ & $\mathbb{z}$ & $\Sigma$ & $\$$ & 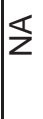 & $\varangle$ & $\varangle$ & 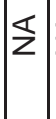 & $\Sigma$ & $\S$ & $\mathbb{z}$ & $\Sigma$ & 7 & 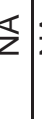 & $\bar{z}$ & $\Sigma \mathbb{z}$ & $\begin{array}{r}\frac{\pi}{2} \\
2\end{array}$ & $\frac{\pi}{z}$ & $\frac{\nwarrow}{z}$ & $\mathbb{Z}$ & 这 \\
\hline 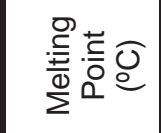 & $\left|\begin{array}{l}1 \\
0 \\
0 \\
\curvearrowright\end{array}\right|$ & $\varangle$ & $\mathbb{z}$ & $\mathbb{z}$ & & $\Sigma$ & $\$$ & 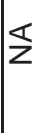 & $\S$ & $\varangle$ & $\varangle$ & $\mathbb{z}$ & $\mathbb{z}$ & § & 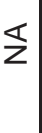 & f & $\S$ & ? & $\Sigma$ & 5 & 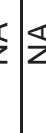 & $\Sigma$ & $\S$ & ¿ \\
\hline 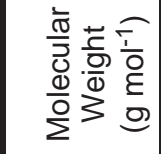 & 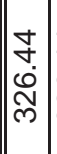 & 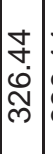 & 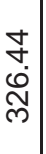 & $\mid$\begin{tabular}{l}
\multirow{2}{*}{} \\
$\dot{0}$ \\
$\mathbb{N}$ \\
$\infty$
\end{tabular} & 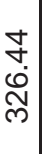 & 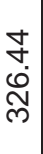 & 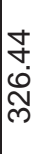 & 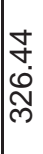 & 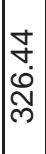 & 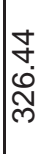 & $\mid \begin{array}{l}\mathbb{J} \\
\dot{N} \\
\tilde{m}\end{array}$ & 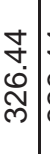 & 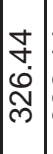 & 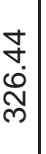 & 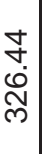 & $\begin{array}{l}\text { \& } \\
\dot{0}\end{array}$ & 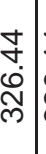 & 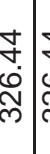 & 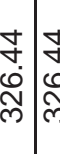 & & & & & 竎 \\
\hline 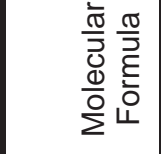 & $\mid \begin{array}{c}\frac{\omega}{0} \\
0 \\
\frac{1}{1} \\
N \\
0 \\
0\end{array}$ & 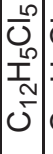 & 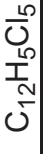 & $\begin{array}{c}\frac{10}{0} \\
0 \\
T \\
N \\
0 \\
0\end{array}$ & 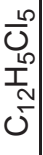 & $\begin{array}{l}\frac{10}{0} \\
\frac{1}{T} \\
T^{N} \\
0\end{array}$ & \begin{tabular}{|l}
$\frac{10}{0}$ \\
0 \\
$\frac{10}{2}$ \\
0 \\
0
\end{tabular} & $\mid \begin{array}{l}\frac{10}{0} \\
0 \\
10 \\
\frac{10}{N} \\
0 \\
0\end{array}$ & $\mid \begin{array}{c}\frac{L}{0} \\
0 \\
\frac{1}{1} \\
N \\
0 \\
0\end{array}$ & 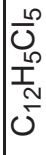 & 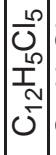 & 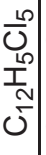 & $\left|\begin{array}{c}\frac{1}{0} \\
0 \\
T \\
N \\
0 \\
0\end{array}\right|$ & 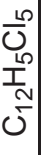 & $\begin{array}{l}\frac{L}{0} \\
\text { L } \\
T^{N} \\
0 \\
0\end{array}$ & $\begin{array}{l}\frac{L}{U} \\
\text { Ln } \\
\text { T. } \\
\text { U. }\end{array}$ & $\begin{array}{l}\frac{\infty}{0} \\
0, \\
\frac{1}{n} \\
0 \\
0\end{array}$ & 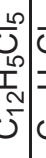 & 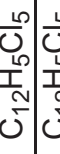 & 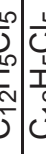 & 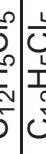 & $\frac{\frac{L}{2}}{0.5}$ & $\mid \begin{array}{l}\frac{10}{0} \\
\frac{1}{20} \\
\frac{1}{2} \\
0 \\
0\end{array}$ & $\frac{10}{\frac{10}{10}}$ \\
\hline $\begin{array}{l}2 \\
2 \\
\infty \\
\text { Tे } \\
0\end{array}$ & $\mid \begin{array}{l}0 \\
\infty \\
\tilde{N} \\
\hat{D} \\
0 \\
0 \\
0 \\
\infty\end{array}$ & $\mid \begin{array}{l}0 \\
0 \\
0 \\
0 \\
1 \\
0 \\
0 \\
0 \\
0\end{array}$ & 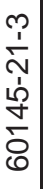 & $\mid$\begin{tabular}{l}
$\infty$ \\
0 \\
0 \\
\hdashline \\
$\infty$ \\
0 \\
0 \\
0 \\
0 \\
1
\end{tabular} & 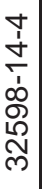 & 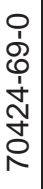 & 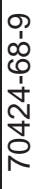 & 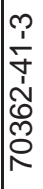 & 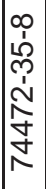 & 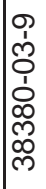 & 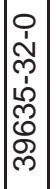 & 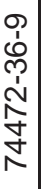 & $\mid$\begin{tabular}{l}
1 \\
0 \\
0 \\
\hdashline \\
$\dot{d}$ \\
$\sigma$ \\
00 \\
0 \\
0
\end{tabular} & 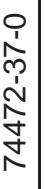 & 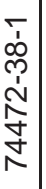 & 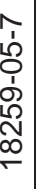 & 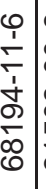 & 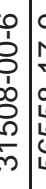 & 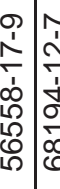 & 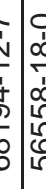 & 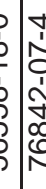 & 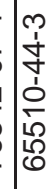 & 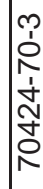 & 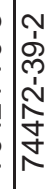 \\
\hline 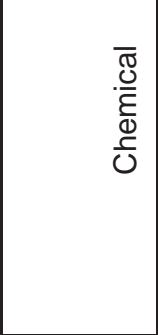 & 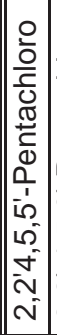 & 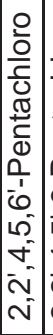 & 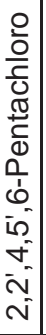 & 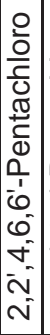 & 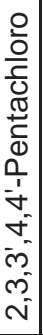 & 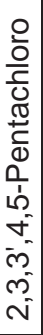 & 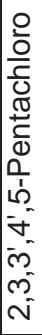 & 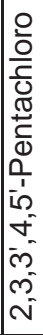 & 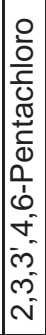 & 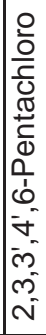 & 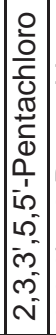 & 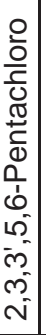 & 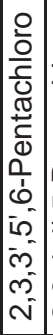 & 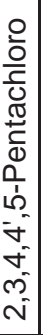 & 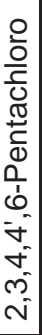 & $\begin{array}{l}\frac{0}{0} \\
\frac{1}{0} \\
\frac{\pi}{0} \\
0 \\
0 \\
0 \\
0 \\
0 \\
0\end{array}$ & 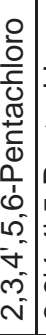 & 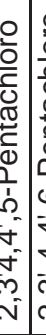 & 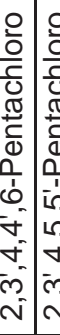 & 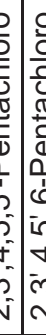 & 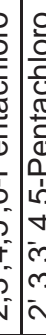 & 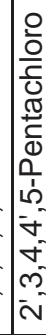 & 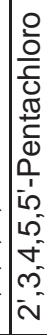 & 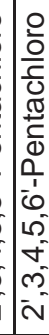 \\
\hline & 흐 & $\approx$ & g & & & & & & & & $\mp$ & $\stackrel{\cong}{\mp}$ & $\stackrel{m}{-1}$ & \pm & $\stackrel{\Omega}{\sim}$ & & $E$ & $\frac{\infty}{\div}$ & $\stackrel{2}{\square}$ & $\vec{v}$ & $\underline{v}$ & 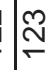 & $\stackrel{+}{\stackrel{\sim}{\sim}}$ & \\
\hline
\end{tabular}




\begin{tabular}{|c|c|c|c|c|c|c|c|c|c|c|c|c|c|c|c|c|c|c|c|c|c|c|c|c|}
\hline 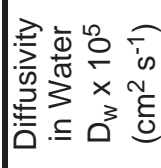 & $\mid \begin{array}{l}\infty \\
\infty \\
0 \\
0 \\
0 \\
0\end{array}$ & & & & $\left|\begin{array}{c}0 \\
0 \\
0 \\
0 \\
0 \\
0\end{array}\right|$ & 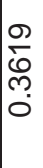 & 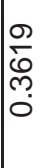 & 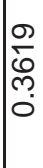 & 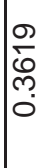 & $\begin{array}{l}\frac{\sigma}{2} \\
0 \\
0 \\
0 \\
0\end{array}$ & $\mid \begin{array}{l}0 \\
0 \\
\tilde{\rho} \\
0 \\
0\end{array}$ & & & & & 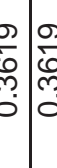 & $\begin{array}{l}\vdots \\
\vdots \\
\vdots \\
\vdots\end{array}$ & & $\mid \begin{array}{l}0 \\
0 \\
0 \\
0 \\
0 \\
0\end{array}$ & & & & & \\
\hline 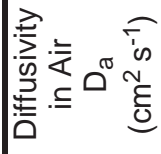 & 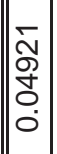 & $\begin{array}{l}\bar{N} \\
\text { Oे } \\
\text { Oे } \\
0\end{array}$ & 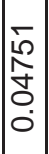 & 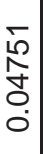 & $\mid$\begin{tabular}{|c|}
- \\
5 \\
0 \\
0 \\
0 \\
0
\end{tabular} & 守 & $\begin{array}{l}1 \\
\frac{1}{2} \\
\text { O } \\
0 \\
0\end{array}$ & $\begin{array}{l}- \\
\frac{1}{2} \\
\text { 年 } \\
0 \\
0\end{array}$ & $\begin{array}{l}5 \\
\frac{1}{2} \\
\text { 年 } \\
0 \\
0\end{array}$ & $\begin{array}{l}5 \\
\frac{1}{2} \\
0 \\
0 \\
0\end{array}$ & 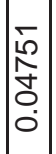 & 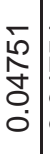 & $\mid$\begin{tabular}{|c|}
1 \\
\\
0 \\
0 \\
0 \\
0
\end{tabular} & $\begin{array}{c}5 \\
\frac{1}{2} \\
0 \\
0 \\
0\end{array}$ & & & 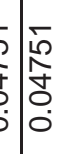 & 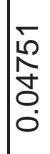 & 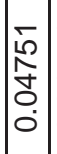 & & $\begin{array}{l}0 \\
0 \\
0 \\
0\end{array}$ & 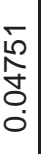 & & 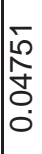 \\
\hline 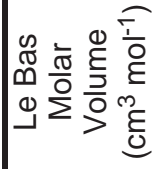 & 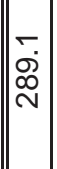 & - & 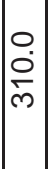 & & $\begin{array}{l}0 \\
\dot{0} \\
\\
m\end{array}$ & $\begin{array}{l}0 \\
\stackrel{0}{0} \\
\frac{0}{m}\end{array}$ & $\begin{array}{l}0 \\
\frac{0}{0} \\
\frac{0}{m}\end{array}$ & 吕 & 奋 & $\begin{array}{l}\frac{0}{0} \\
\frac{0}{m}\end{array}$ & $\begin{array}{l}0 \\
\stackrel{0}{0} \\
\bar{m}\end{array}$ & & & & & & : & & $\begin{array}{l}0 \\
0 \\
0 \\
m\end{array}$ & & & & & \\
\hline 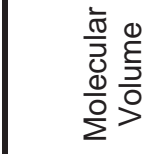 & $\left.\mid \begin{array}{l}\infty \\
0 \\
0 \\
N\end{array}\right)$ & 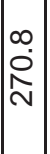 & 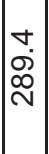 & $\begin{array}{l}\stackrel{\leftrightarrow}{.} \\
\stackrel{D}{\sim} \\
\stackrel{D}{0}\end{array}$ & 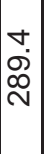 & 衣 & 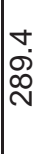 & 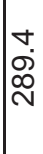 & 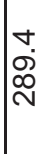 & 离 & 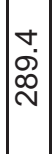 & 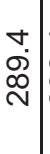 & 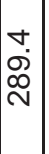 & 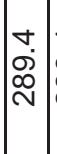 & 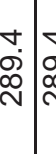 & 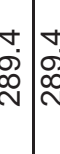 & : & 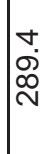 & 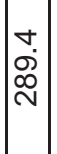 & 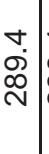 & 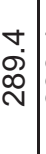 & 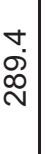 & & \\
\hline 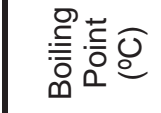 & $\Sigma$ & $\Sigma$ & 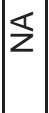 & 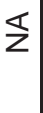 & $\mathbb{z}$ & $\Sigma$ & 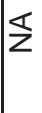 & 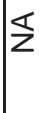 & 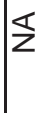 & 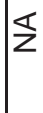 & 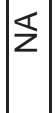 & $\mathbb{Z}$ & 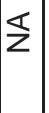 & $\mathbb{Z}$ & $\Sigma$ & $\mathbb{z} \mid \mathbb{z}$ & 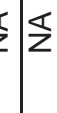 & & 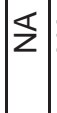 & 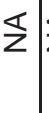 & $\Sigma$ & $\S$ & & 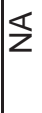 \\
\hline 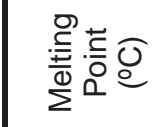 & $\Sigma$ & 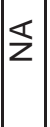 & $\begin{array}{l}0 \\
0 \\
0 \\
10 \\
-1\end{array}$ & $\begin{array}{l}0 \\
\dot{\rho} \\
\infty \\
\infty\end{array}$ & $\$$ & $\$$ & $\$$ & $\$$ & 웅 & $\S$ & 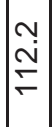 & 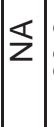 & $\begin{array}{l}0 \\
\dot{\infty} \\
\dot{\infty}\end{array}$ & 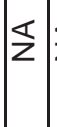 & $\Sigma$ & $\mathbb{z}$ & $\sum \Sigma$ & z & $\S$ & 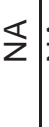 & $\Sigma$ & 乏 & & $\varangle$ \\
\hline 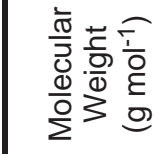 & 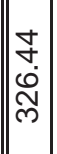 & \begin{tabular}{l}
\multirow{4}{*}{} \\
$\stackrel{0}{0}$ \\
$\tilde{\omega}$
\end{tabular} & $\mid \begin{array}{l}\infty \\
\infty \\
0 \\
0 \\
\infty\end{array}$ & & $\mid \begin{array}{l}\infty \\
\infty \\
0 \\
0 \\
0 \\
\infty\end{array}$ & $\begin{array}{l}\infty \\
\infty \\
\infty \\
0 \\
0 \\
\infty\end{array}$ & $\begin{array}{l}\infty \\
\infty \\
\infty \\
\dot{0} \\
\infty \\
\infty\end{array}$ & 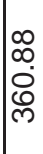 & $\begin{array}{l}\infty \\
\infty \\
\infty \\
0 \\
0 \\
0\end{array}$ & $\begin{array}{l}\infty \\
\infty \\
0 \\
0 \\
0 \\
0\end{array}$ & $\mid \begin{array}{l}\infty \\
\infty \\
0 \\
0 \\
\infty\end{array}$ & $\mid \begin{array}{l}\infty \\
\infty \\
0 \\
0 \\
0 \\
0\end{array}$ & $\mid$ & & & & $\begin{array}{l}0 \\
\vdots \\
0\end{array}$ & . & $\mid \begin{array}{l}\infty \\
\infty \\
0 \\
0 \\
0\end{array}$ & $\mid \begin{array}{l}\infty \\
\infty \\
0 \\
0 \\
0 \\
\infty\end{array}$ & $\begin{array}{l}\infty \\
\infty \\
0 \\
0 \\
ల\end{array}$ & $\begin{array}{l}\infty \\
\infty \\
0 \\
0 \\
ల\end{array}$ & & $\begin{array}{l}\infty \\
\infty \\
0 \\
0 \\
\infty \\
ల\end{array}$ \\
\hline 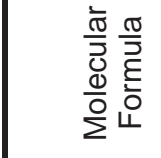 & 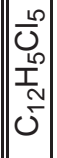 & 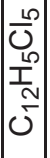 & 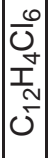 & $\begin{array}{l}0 \\
0 \\
I^{0} \\
I^{N} \\
0\end{array}$ & $\mid \begin{array}{c}0 \\
0 \\
I^{+} \\
N \\
0 \\
0\end{array}$ & $\mid \begin{array}{l}0 \\
0 \\
J^{\prime} \\
I^{N} \\
0 \\
0\end{array}$ & $\mid \begin{array}{l}0 \\
0 \\
I^{\prime} \\
I^{N} \\
0 \\
0\end{array}$ & 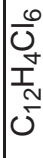 & $\begin{array}{l}0 \\
0 \\
I^{+} \\
I^{n} \\
0 \\
0\end{array}$ & $\mid \begin{array}{l}0 \\
0 \\
I^{\prime} \\
N \\
0 \\
0\end{array}$ & 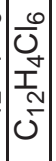 & 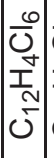 & 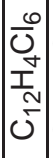 & $\left|\begin{array}{c}0 \\
0 \\
T^{+} \\
\underline{N} \\
0 \\
0\end{array}\right|$ & 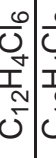 & 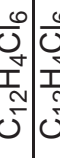 & 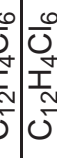 & $=\mid \begin{array}{l}0 \\
0 \\
U^{+} \\
U^{N}\end{array}$ & $\left|\begin{array}{c}0 \\
0 \\
T^{0} \\
\\
0 \\
0\end{array}\right|$ & 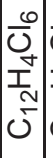 & $\begin{array}{l}0 \\
0 \\
I^{\prime} \\
\tilde{N} \\
\mathcal{N}\end{array}$ & $\mid \begin{array}{c}0 \\
0 \\
I^{0} \\
I^{2} \\
0 \\
0\end{array}$ & & $\begin{array}{l}0 \\
0 \\
I^{\prime} \\
I^{N} \\
0\end{array}$ \\
\hline 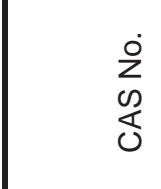 & $\mid \begin{array}{l}0 \\
0 \\
0 \\
0 \\
N \\
1 \\
0 \\
0 \\
1 \\
1 \\
0\end{array}$ & 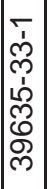 & $\begin{array}{l}0 \\
0 \\
i \\
0 \\
0 \\
\infty \\
0 \\
0 \\
\infty \\
m\end{array}$ & 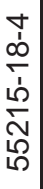 & 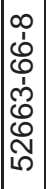 & 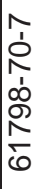 & 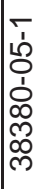 & 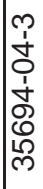 & 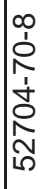 & 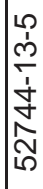 & 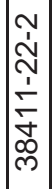 & 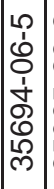 & 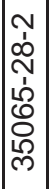 & $\begin{array}{l}0 \\
0 \\
0 \\
1 \\
0 \\
0 \\
0 \\
0 \\
1 \\
1\end{array}$ & 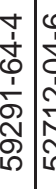 & 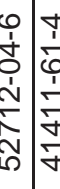 & 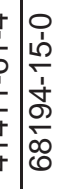 & 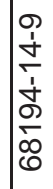 & 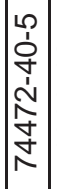 & $\begin{array}{l}\infty \\
0 \\
0 \\
0 \\
0 \\
0 \\
0 \\
\vdots \\
i n\end{array}$ & 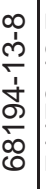 & 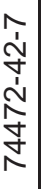 & & 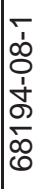 \\
\hline $\begin{array}{l}\frac{\pi}{\frac{0}{\varepsilon}} \\
\frac{0}{0} \\
\frac{\pi}{U}\end{array}$ & 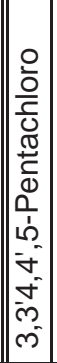 & 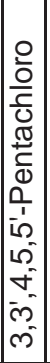 & 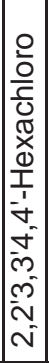 & 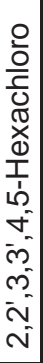 & 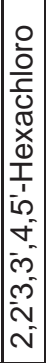 & 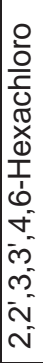 & 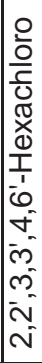 & 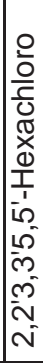 & 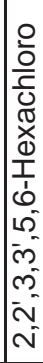 & 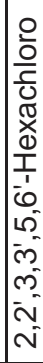 & 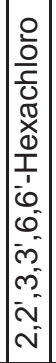 & 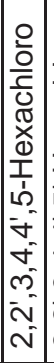 & 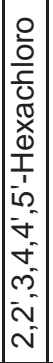 & 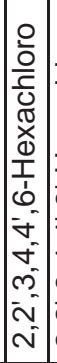 & 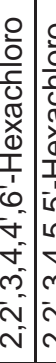 & 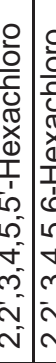 & 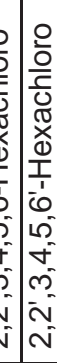 & 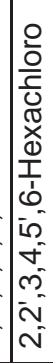 & 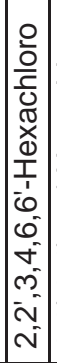 & 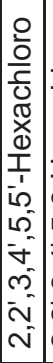 & 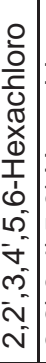 & 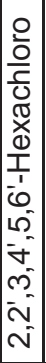 & & 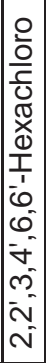 \\
\hline & $\| \stackrel{\leftrightarrow}{N}$ & & & & & & & & & & लि & & & & & $\dot{ \pm} \mid q$ & \begin{tabular}{l}
$y$ \\
\hdashline \\
\end{tabular} & & 过 & & & & & \\
\hline
\end{tabular}




\begin{tabular}{|c|c|c|c|c|c|c|c|c|c|c|c|c|c|c|c|c|c|c|c|c|c|c|c|}
\hline 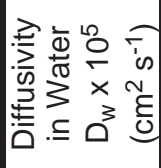 & $\mid \begin{array}{l}0 \\
0 \\
m \\
0 \\
0\end{array}$ & & $\left|\begin{array}{l}0 \\
0 \\
0 \\
0 \\
0 \\
0\end{array}\right|$ & $\begin{array}{l}\frac{\pi}{0} \\
\overline{0} \\
\text { o. } \\
0\end{array}$ & $\begin{array}{l}\sigma \\
\overline{0} \\
\text { en } \\
0 \\
0\end{array}$ & $\begin{array}{l}0 \\
0 \\
0 \\
0 \\
0\end{array}$ & $\mid \begin{array}{l}0 \\
0 \\
0 \\
0 \\
0 \\
0\end{array}$ & 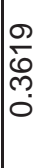 & 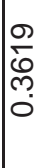 & $\begin{array}{l}\sigma \\
\frac{0}{0} \\
0 \\
0 \\
0\end{array}$ & $\left|\begin{array}{l}0 \\
0 \\
0 \\
0 \\
0\end{array}\right|$ & $\begin{array}{c}0 \\
\\
0 \\
0 \\
0\end{array}$ & & & & 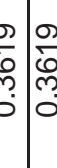 & 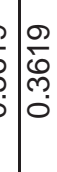 & & & & & & \\
\hline 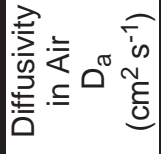 & $\mid \begin{array}{c}- \\
5 \\
0 \\
0 \\
0\end{array}$ & 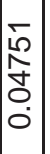 & 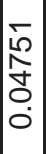 & $\begin{array}{l}5 \\
5 \\
5 \\
0 \\
0\end{array}$ & $\begin{array}{l}5 \\
5 \\
0 \\
0 \\
0\end{array}$ & $\begin{array}{l}8 \\
0 \\
0 \\
0\end{array}$ & $\begin{array}{l}- \\
5 \\
0 \\
0 \\
0\end{array}$ & $\begin{array}{l}-5 \\
5 \\
0 \\
0 \\
0 \\
0\end{array}$ & $\begin{array}{l}5 \\
\frac{1}{2} \\
\text { Oे } \\
0 \\
0\end{array}$ & $\begin{array}{l}-5 \\
\frac{1}{2} \\
\text { 年 } \\
0 \\
0\end{array}$ & 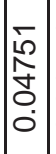 & $\begin{array}{l}- \\
5 \\
5 \\
0 \\
0\end{array}$ & 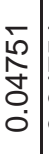 & $\mid \begin{array}{ll}- \\
0 \\
0 \\
0 \\
0 \\
0 \\
0 \\
0\end{array}$ & & & $\begin{array}{l}5 \\
\frac{1}{2} \\
0 \\
0 \\
0\end{array}$ & $\mid \begin{array}{l}\bar{n} \\
\frac{1}{2} \\
\dot{0} \\
0 \\
0\end{array}$ & $\begin{array}{l}0 \\
0 \\
0 \\
0 \\
0\end{array}$ & 容 & & & \\
\hline 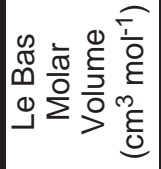 & 奋 & & $\frac{0}{0}$ & & & $\begin{array}{l}0 \\
\dot{0} \\
\frac{0}{m}\end{array}$ & $\begin{array}{l}0 \\
\dot{0} \\
\\
\end{array}$ & $\begin{array}{l}\frac{0}{0} \\
\frac{0}{m} \\
\text { m. }\end{array}$ & $\begin{array}{l}0.0 \\
\frac{0}{m} \\
\frac{0}{m}\end{array}$ & $\begin{array}{l}0 \\
0 \\
\text { o. } \\
\text { m }\end{array}$ & $\begin{array}{l}0 \\
0 \\
0 \\
\end{array}$ & $\begin{array}{l}0 \\
\dot{0} \\
\frac{0}{m}\end{array}$ & & & & & : & & & 。্ & & & \\
\hline 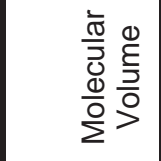 & $\begin{array}{l}\forall \\
\stackrel{\infty}{ } \\
\stackrel{\infty}{N}\end{array}$ & $\left|\begin{array}{l}\forall \\
\mathbb{D} \\
\mathbb{\infty} \\
\sim\end{array}\right|$ & 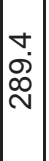 & 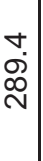 & $\begin{array}{l}\dot{D} \\
\stackrel{D}{\infty} \\
\stackrel{\sim}{\sim}\end{array}$ & 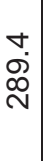 & 宓 & 㐫 & 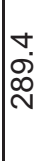 & 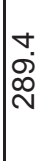 & 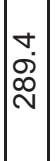 & 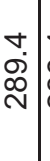 & $\begin{array}{l}\stackrel{\nabla}{.} \\
\stackrel{\sim}{\sim}\end{array}$ & 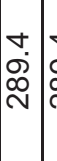 & 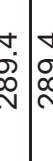 & 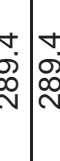 & 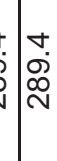 & 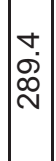 & $\begin{array}{c}- \\
\infty \\
\\
\end{array}$ & ;) & 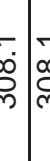 & 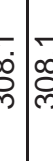 & \\
\hline 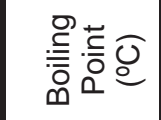 & $\mathbb{z}$ & $\mathbb{z}$ & 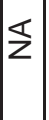 & $\mathbb{z}$ & $\mathbb{z}$ & $\Sigma$ & $\$$ & 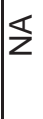 & 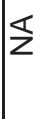 & 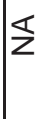 & 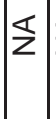 & $\Sigma$ & $\S$ & $\Sigma$ & & $\mathbb{z} \mid \mathbb{z}$ & 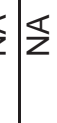 & 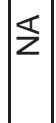 & $\Sigma$ & $\Sigma$ & $\begin{array}{r}\mathbf{z} \\
\mathbf{z}\end{array}$ & $\bar{z}$ & 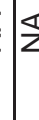 \\
\hline 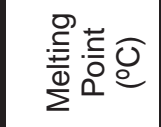 & $\S$ & $\mathbb{z}$ & 일 & $\mathbb{z}$ & $\begin{array}{l}0 \\
\dot{+} \\
\leftarrow\end{array}$ & $\Sigma$ & 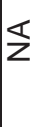 & $\S$ & $\S$ & $\varangle$ & $\varangle$ & $\Sigma$ & $\Sigma$ & $\S$ & 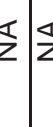 & $\mathbb{z} \mid \mathbf{z}$ & 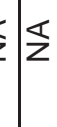 & 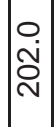 & 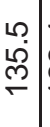 & $\begin{array}{l}\text { Ṅ } \\
\text { Ṅ}\end{array}$ & Z & $\frac{4}{2}$ & 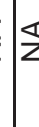 \\
\hline 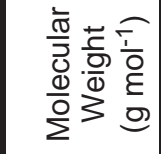 & $\mid \begin{array}{l}\infty \\
\infty \\
0 \\
0 \\
\infty \\
\infty\end{array}$ & $\begin{array}{l}\infty \\
\infty \\
0 \\
0 \\
0 \\
0\end{array}$ & $\mid \begin{array}{l}\infty \\
0 \\
0 \\
0 \\
0 \\
0\end{array}$ & $\begin{array}{c}\infty \\
\infty \\
0 \\
0 \\
\infty \\
\infty\end{array}$ & $\begin{array}{l}\infty \\
\infty \\
0 \\
0 \\
0 \\
\text { D. }\end{array}$ & $\begin{array}{l}\infty \\
\infty \\
0 \\
0 \\
\infty \\
\text {. }\end{array}$ & $\begin{array}{l}\infty \\
\infty \\
\infty \\
0 \\
0 \\
\infty\end{array}$ & $\begin{array}{l}\infty \\
\infty \\
\infty \\
0 \\
\infty \\
\infty\end{array}$ & $\begin{array}{l}\infty \\
\infty \\
\infty \\
0 \\
\infty \\
\infty\end{array}$ & 舟 & $\mid$ & $\mid \begin{array}{l}\infty \\
\infty \\
\infty \\
0 \\
0 \\
m\end{array}$ & $\mid \begin{array}{l}\infty \\
\infty \\
0 \\
0 \\
\infty \\
m\end{array}$ & & & & 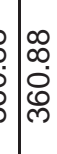 & $\mid \begin{array}{l}\infty \\
\infty \\
0 \\
0 \\
\infty \\
\infty\end{array}$ & 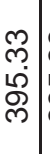 & 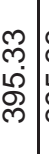 & & & \\
\hline 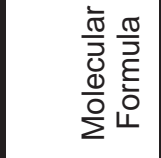 & $\left|\begin{array}{c}0 \\
0 \\
0 \\
I \\
N \\
0 \\
0 \\
N\end{array}\right|$ & & 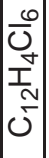 & 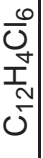 & $\begin{array}{l}0 \\
\\
I^{0} \\
I^{N} \\
0\end{array}$ & 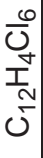 & \begin{tabular}{|l}
0 \\
0 \\
$I^{\prime}$ \\
$N$ \\
0 \\
0
\end{tabular} & $\mid \begin{array}{l}0 \\
0 \\
I^{+} \\
I^{N} \\
0 \\
0\end{array}$ & $\mid \begin{array}{l}0 \\
0 \\
I^{+} \\
I^{2} \\
0 \\
0\end{array}$ & $\mid \begin{array}{l}0 \\
0 \\
\\
I \\
N \\
j \\
j\end{array}$ & 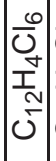 & $\mid \begin{array}{c}0 \\
0 \\
I^{+} \\
N \\
0 \\
0\end{array}$ & $\left|\begin{array}{c}0 \\
0 \\
I^{+} \\
N \\
0 \\
0\end{array}\right|$ & $\left|\begin{array}{c|c}0 \\
0 \\
\\
I \\
N \\
\tilde{N} \\
0 \\
0\end{array}\right|$ & & 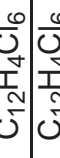 & 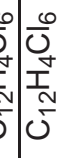 & 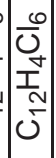 & $\begin{array}{l}\hat{N} \\
0 \\
\Lambda \\
N \\
0\end{array}$ & $\begin{array}{l}\bar{N} \\
\mathcal{m}^{N} \\
\bar{N}\end{array}$ & 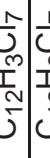 & 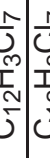 & 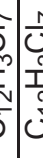 \\
\hline $\begin{array}{l}20 \\
\text { ¿n } \\
\text { Q }\end{array}$ & 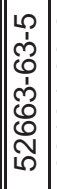 & 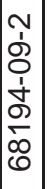 & 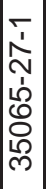 & 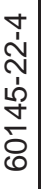 & 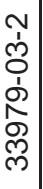 & $\begin{array}{l}0 \\
\dot{1} \\
0 \\
0 \\
0 \\
0 \\
0 \\
0 \\
0 \\
0 \\
0\end{array}$ & $\begin{array}{l}\hat{1} \\
\dot{0} \\
0 \\
\dot{1} \\
\infty \\
0 \\
0 \\
0 \\
0\end{array}$ & 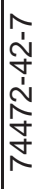 & 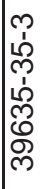 & 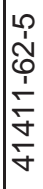 & 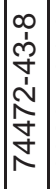 & 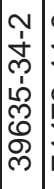 & 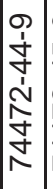 & 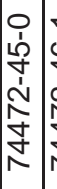 & 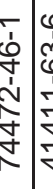 & 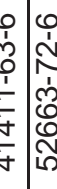 & 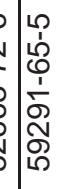 & $\begin{array}{l}0 \\
0 \\
0 \\
1 \\
d \\
N \\
\hat{d} \\
\tilde{N}\end{array}$ & 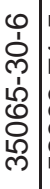 & 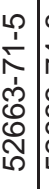 & 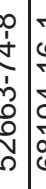 & 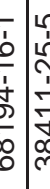 & 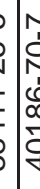 \\
\hline 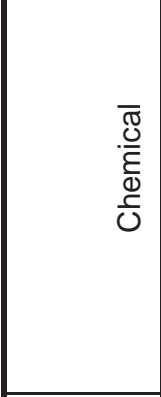 & 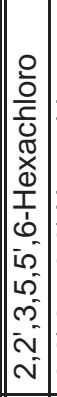 & 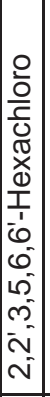 & 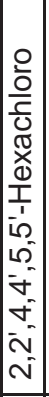 & 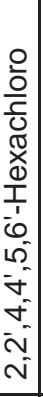 & 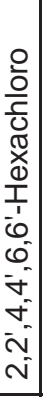 & 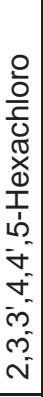 & 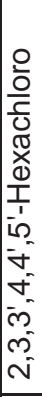 & 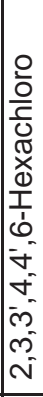 & 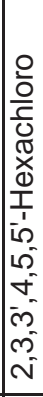 & 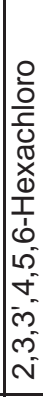 & 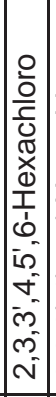 & 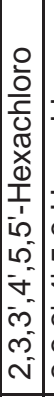 & 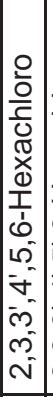 & 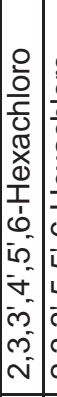 & 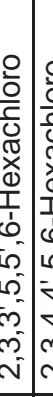 & 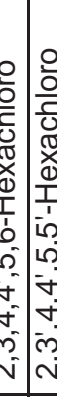 & 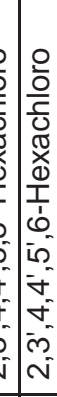 & 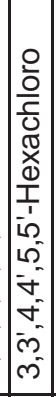 & 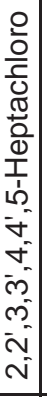 & 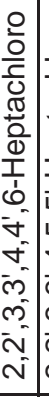 & 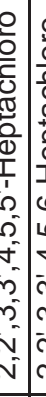 & 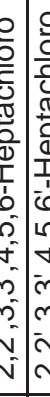 & 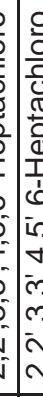 \\
\hline 으 & $\mid \underline{5}$ & $\underline{\tilde{N}}$ & & & & & & L & & & $\overline{0}$ & 6 & అ్రి & षै & ๑ొ & : & $\dot{1}$ & $\stackrel{8}{\varrho}$ & $\stackrel{\Sigma}{\Sigma}$ & & 7 & ? & \\
\hline
\end{tabular}




\begin{tabular}{|c|c|c|c|c|c|c|c|c|c|c|c|c|c|c|c|c|c|c|c|c|c|c|c|}
\hline 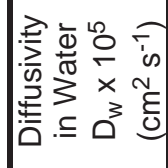 & $\mid \begin{array}{c}- \\
\infty \\
\infty \\
0 \\
0\end{array}$ & $\mid$ & $\mid \begin{array}{c}-\infty \\
\stackrel{N}{ } \\
m \\
0 \\
0\end{array}$ & & & 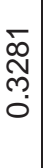 & 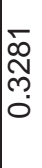 & 免 & 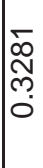 & 免 & 柋 & 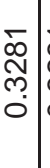 & $\begin{array}{c}\bar{\infty} \\
\stackrel{\infty}{ } \\
\text { o. } \\
\dot{0}\end{array}$ & & & 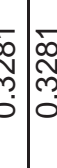 & 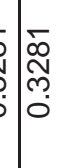 & 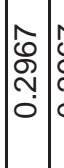 & & & & & \\
\hline 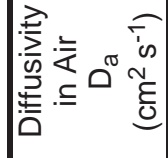 & $\mid \begin{array}{c}\hat{\alpha} \\
0 \\
0 \\
0 \\
0 \\
0\end{array}$ & $\mid \begin{array}{l}1 \\
0 \\
0 \\
0 \\
0 \\
0\end{array}$ & 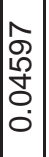 & & & $\begin{array}{l}1 \\
0 \\
0 \\
0 \\
0 \\
0\end{array}$ & $\begin{array}{l}1 \\
\text { Oे } \\
0 \\
0 \\
0 \\
0\end{array}$ & 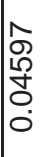 & $\begin{array}{l}\hat{\beta} \\
\text { r } \\
0 \\
0 \\
0\end{array}$ & $\mid \begin{array}{l}1 \\
0 \\
i \\
0 \\
0 \\
0\end{array}$ & 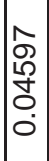 & $\begin{array}{l}1 \\
0 \\
0 \\
0 \\
0 \\
0\end{array}$ & $\begin{array}{l}0 \\
\dot{0} \\
0 \\
0 \\
0 \\
0\end{array}$ & & & 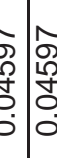 & bे & 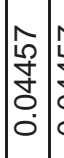 & 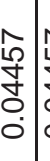 & & & & 3 \\
\hline 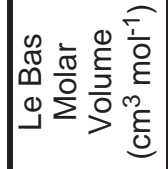 & 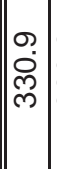 & 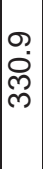 & 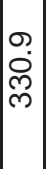 & & & 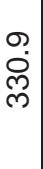 & 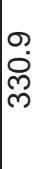 & 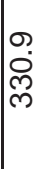 & 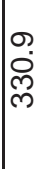 & 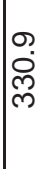 & 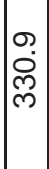 & 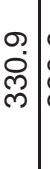 & 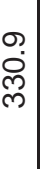 & & & & ?. & & & & & & \\
\hline 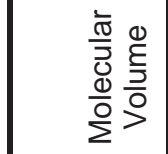 & $\begin{array}{l}- \\
\infty \\
0 \\
0\end{array}$ & $\mid \begin{array}{l}- \\
\infty \\
0 \\
\infty \\
\end{array}$ & - & $\begin{array}{l}- \\
\infty \\
0 \\
0\end{array}$ & $\begin{array}{l}- \\
\infty \\
\stackrel{\rho}{\circ}\end{array}$ & - & - & - & - & - & - & $\begin{array}{c}- \\
\infty \\
\infty \\
\infty\end{array}$ & - & 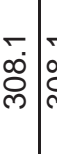 & & 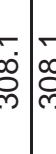 & bे & 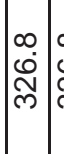 & 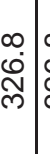 & 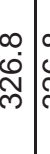 & 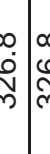 & & 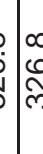 \\
\hline 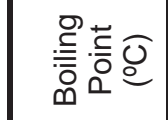 & 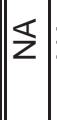 & $\$$ & $\Sigma$ & $\Sigma$ & $\mathbb{z}$ & $\Sigma$ & $\$$ & 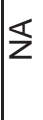 & 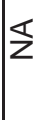 & 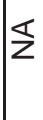 & z & $\Sigma$ & ¿ & $\mathbb{z}$ & & $\mathbb{z} \leq$ & 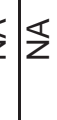 & & $\Sigma$ & $\Sigma$ & $\begin{array}{l}\mathbf{z} \\
\mathrm{z}\end{array}$ & $\mathbb{Z}$ & $\bar{Z}$ \\
\hline 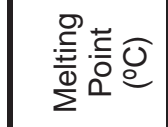 & $\varangle$ & $\Sigma$ & $\Sigma$ & $\Sigma$ & & $\Sigma$ & $\Sigma$ & $\S$ & $\$$ & 宲 & $\Sigma$ & $\mathbb{Z}$ & $\mathbb{Z}$ & & 齐 & $\mathbb{z}$ & $\S$ & 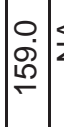 & $\S$ & $\Sigma$ & Z & $\frac{\mathbb{s}}{z}$ & 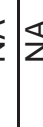 \\
\hline 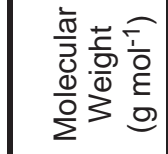 & 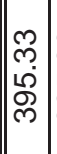 & 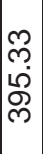 & 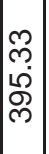 & & 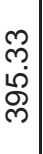 & 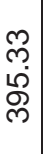 & 孞 & 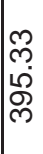 & 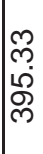 & 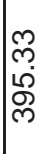 & $\begin{array}{l}\tilde{m} \\
\tilde{m} \\
\mathscr{m} \\
\tilde{m}\end{array}$ & 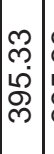 & 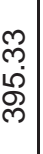 & & & & p. & 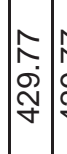 & 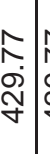 & 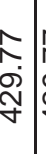 & & & 方 \\
\hline 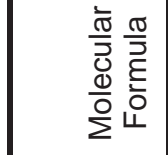 & 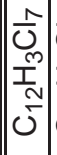 & & \begin{tabular}{|l}
$N$ \\
0 \\
$\mathbb{M}$ \\
$\underline{N}$ \\
$\tilde{N}$
\end{tabular} & 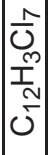 & 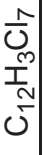 & $\begin{array}{l}\frac{N}{0} \\
N_{1}^{N} \\
U^{N}\end{array}$ & $\begin{array}{l}\frac{N}{0} \\
T^{2} \\
N^{N} \\
0\end{array}$ & 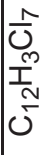 & 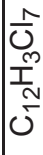 & 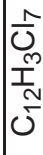 & $\begin{array}{l}\frac{1}{0} \\
\mathbb{m} \\
\underline{N} \\
\tilde{J}\end{array}$ & $\mid \begin{array}{c}\hat{N} \\
0 \\
\underline{N} \\
\mathcal{N}\end{array}$ & 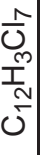 & 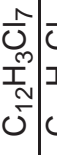 & 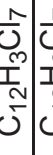 & 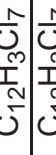 & 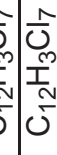 & 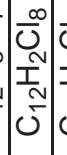 & 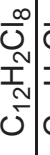 & $\begin{array}{c}\infty \\
0 \\
N_{1}^{\infty} \\
0 \\
0\end{array}$ & 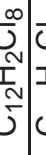 & 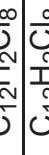 & 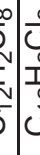 \\
\hline $\begin{array}{l}2 \\
2 \\
0 \\
0 \\
0\end{array}$ & $\mid \begin{array}{l}1 \\
1 \\
0 \\
0 \\
\infty \\
0 \\
0 \\
\omega \\
\hat{n}\end{array}$ & 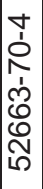 & 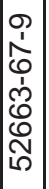 & $\begin{array}{l}0 \\
1 \\
0 \\
0 \\
0 \\
0 \\
0 \\
\tilde{N} \\
1\end{array}$ & 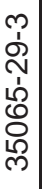 & 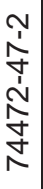 & 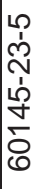 & 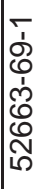 & 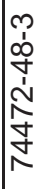 & 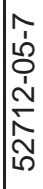 & 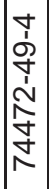 & 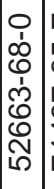 & 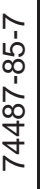 & 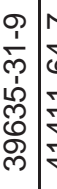 & 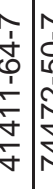 & 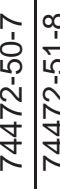 & 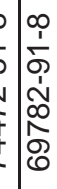 & 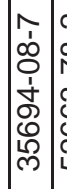 & 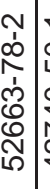 & 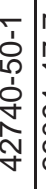 & 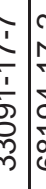 & 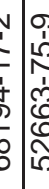 & 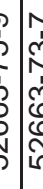 \\
\hline 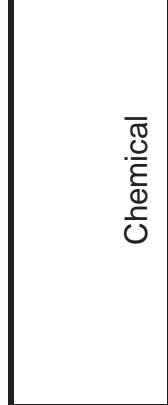 & 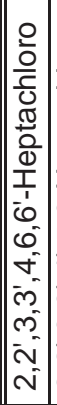 & 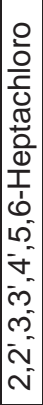 & 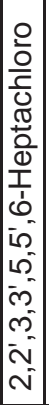 & 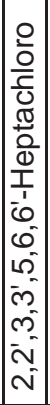 & 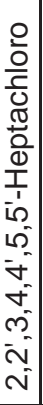 & 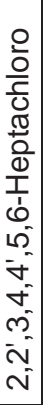 & 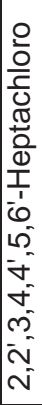 & 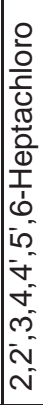 & 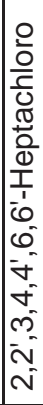 & 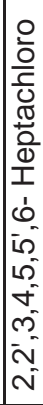 & 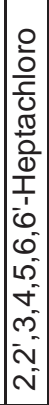 & 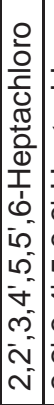 & 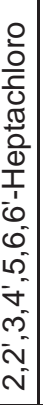 & 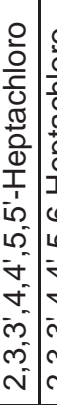 & 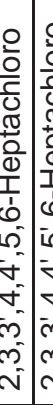 & 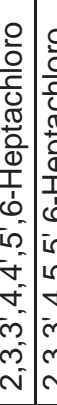 & 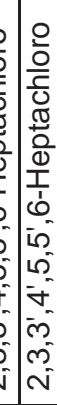 & 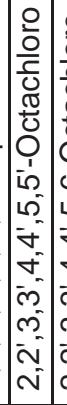 & 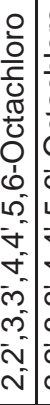 & 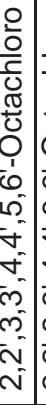 & 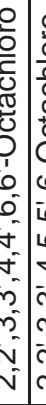 & 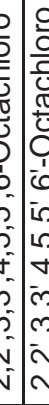 & 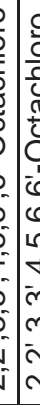 \\
\hline & $\infty$ & & & & & & & & & & $\infty$ & & & \&) & & $\bar{\sigma} \sigma \frac{0}{\sigma}$ & |ळ & Ðே & & & & & \\
\hline
\end{tabular}




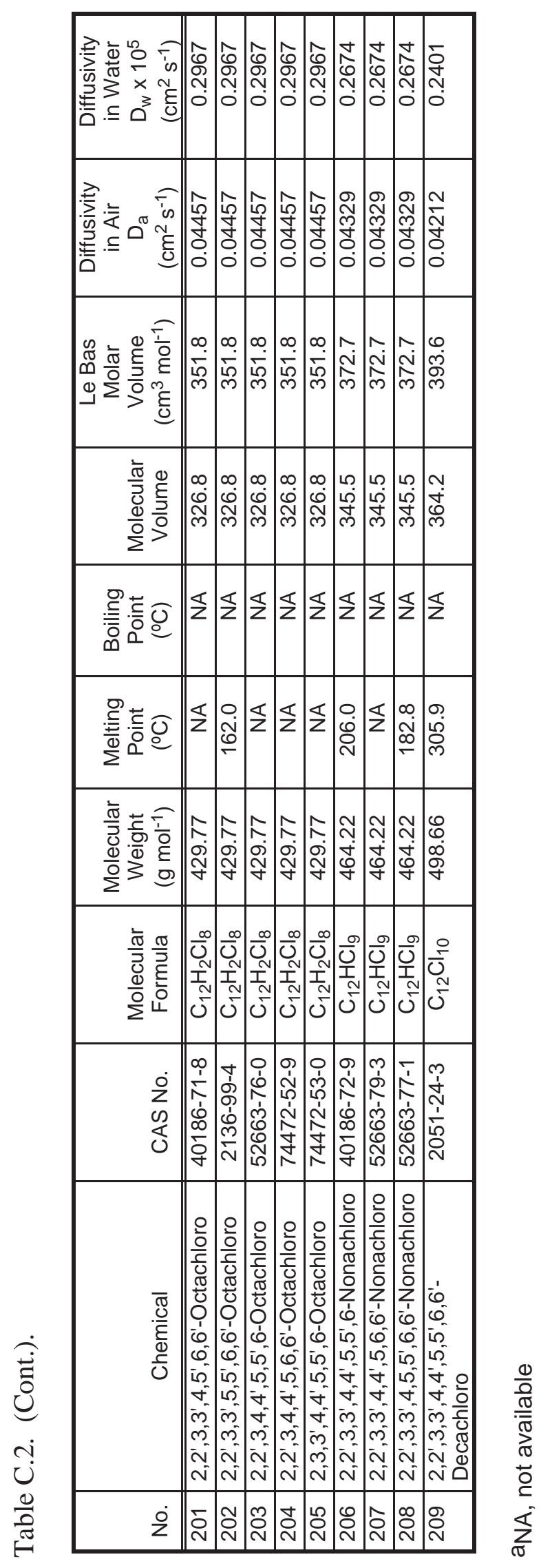




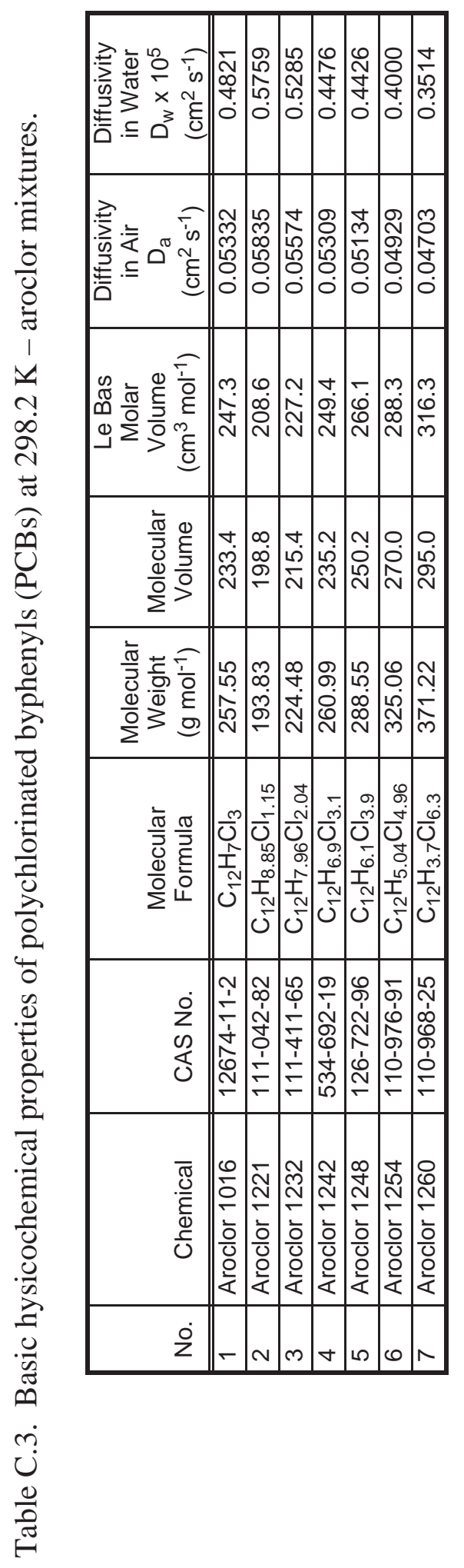

$\frac{\infty}{U^{\prime}}$ 


\begin{tabular}{|c|c|c|c|c|c|c|c|c|c|c|c|c|c|c|c|}
\hline 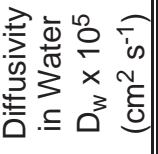 & $\mid \begin{array}{l}\infty \\
6 \\
0 \\
0 \\
0 \\
0\end{array}$ & 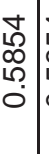 & \begin{tabular}{c|c}
0 \\
0 \\
0 \\
0 \\
0 \\
0
\end{tabular} & 旡 & 占 & 占 & 府 & ๙ิ & 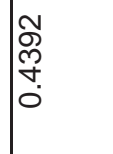 & 凩 & 令 & लె & 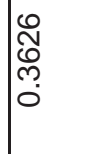 & 成 & $\begin{array}{l}\text { N } \\
\hat{N} \\
\text { N̦ } \\
0\end{array}$ \\
\hline 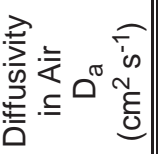 & $\mid \begin{array}{c}0 \\
0 \\
\dot{f} \\
0 \\
0 \\
0\end{array}$ & 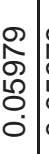 & 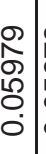 & $\begin{array}{l}\infty \\
1 \\
0 \\
0 \\
0 \\
0 \\
0\end{array}$ & $\begin{array}{l}\infty \\
1 \\
0 \\
0 \\
0 \\
0 \\
0\end{array}$ & $\begin{array}{l}\infty \\
0 \\
0 \\
0 \\
0 \\
0 \\
0\end{array}$ & 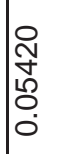 & $\begin{array}{l}0 \\
0 \\
\frac{1}{2} \\
0 \\
0 \\
0 \\
0\end{array}$ & 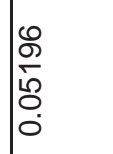 & 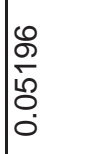 & 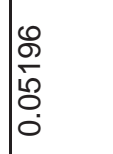 & $\begin{array}{l}\infty \\
\stackrel{\infty}{\circ} \\
\text { क्ष } \\
0 \\
0\end{array}$ & $\begin{array}{l}\tilde{N} \\
\infty \\
0 \\
0 \\
0 \\
0\end{array}$ & $\mid \begin{array}{l}0 \\
0 \\
0 \\
0 \\
0 \\
0 \\
0\end{array}$ & $\begin{array}{l}\frac{0}{10} \\
\frac{10}{0} \\
0 \\
0\end{array}$ \\
\hline 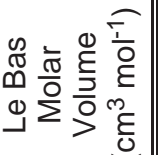 & $\mid \begin{array}{c} \\
\sim \\
\sim \\
\infty \\
0 \\
- \\
\end{array}$ & - & С્ & $\begin{array}{l}0 \\
\stackrel{0}{N} \\
\text { N }\end{array}$ & $\begin{array}{l}0 \\
\dot{\mathfrak{N}} \\
\text { N }\end{array}$ & $\begin{array}{l}0 \\
\stackrel{0}{ } \\
\text { N }\end{array}$ & or & $\begin{array}{l}\infty \\
N \\
\stackrel{\omega}{N} \\
N\end{array}$ & $\begin{array}{l}\infty \\
\hat{N} \\
\stackrel{\infty}{N}\end{array}$ & $\begin{array}{l}\infty \\
i \\
\stackrel{\omega}{N} \\
N\end{array}$ & $\begin{array}{l}\infty \\
\hat{N} \\
\stackrel{N}{N}\end{array}$ & $\begin{array}{l}\hat{N} \\
\infty \\
\infty \\
\sim\end{array}$ & $\begin{array}{l}0 \\
\dot{0} \\
0\end{array}$ & 售 & $\frac{\dot{v}}{\dot{\infty}}$ \\
\hline 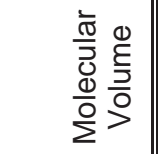 & $\begin{array}{l}0 \\
0 \\
0 \\
0 \\
-1\end{array}$ & $\begin{array}{c}\infty \\
\infty \\
\infty \\
\infty\end{array}$ & $\begin{array}{l}\infty \\
\infty \\
\infty \\
\infty\end{array}$ & O & O̊ & O̊. & Nิָ̃ & 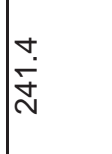 & 芦 & $\mid \frac{a}{\dot{d}}$ & $\frac{\text { d. }}{\stackrel{d}{d}}$ & $\check{\grave{Q}}$ & $\begin{array}{l}\hat{N} \\
\infty \\
\stackrel{\infty}{N}\end{array}$ & సি. & $\frac{\bar{\omega}}{\frac{\omega}{m}}$ \\
\hline 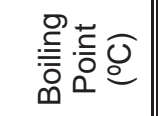 & $\mid \begin{array}{l}\infty \\
\infty \\
\infty \\
\sim \\
\infty \\
\infty\end{array}$ & $\begin{array}{l}n \\
\frac{\rho}{m} \\
\end{array}$ & $\begin{array}{l}0 \\
\dot{0} \\
\frac{m}{m}\end{array}$ & o & 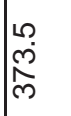 & $\stackrel{\infty}{\Sigma}$ & 吕 & $\frac{0}{\partial}$ & $\begin{array}{l}m \\
\infty \\
\dddot{\rho} \\
q\end{array}$ & $\begin{array}{l}m \\
\infty \\
\infty \\
\tilde{q}\end{array}$ & $\mid \begin{array}{l}10 \\
0 \\
0 \\
\sigma \\
\end{array}$ & 家 & 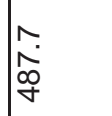 & $\begin{array}{l}\text { nִ } \\
\text { No } \\
\text { n }\end{array}$ & $\begin{array}{l}0 \\
0 \\
\text { in }\end{array}$ \\
\hline 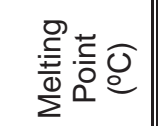 & 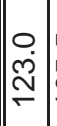 & $\begin{array}{l}0 \\
\stackrel{0}{0} \\
0 \\
\end{array}$ & $\begin{array}{l}0 \\
\dot{a} \\
\infty\end{array}$ & $\begin{array}{l}0 \\
\dot{0} \\
0\end{array}$ & 웅 & $\begin{array}{l}0 \\
\frac{1}{10} \\
1\end{array}$ & 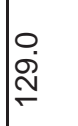 & 웅 & $\begin{array}{l}0 \\
\stackrel{N}{N} \\
\end{array}$ & $\begin{array}{l}\circ \\
\stackrel{0}{N}\end{array}$ & $\begin{array}{l}\text { o } \\
\text { ம் } \\
\text { p' }\end{array}$ & 옴 & $\begin{array}{l}\circ \\
\stackrel{0}{N} \\
\text { N }\end{array}$ & $\begin{array}{l}0 \\
\text { in } \\
\stackrel{\leftrightarrow}{\text { N }}\end{array}$ & $\begin{array}{l}\text { 오 } \\
\text { స్ }\end{array}$ \\
\hline 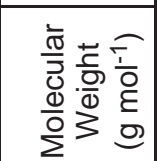 & 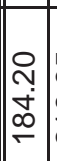 & $\begin{array}{l}0 \\
0 \\
\infty \\
\\
\end{array}$ & $\begin{array}{l}\infty \\
0 \\
\infty \\
\\
\end{array}$ & 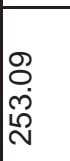 & 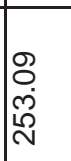 & 呑 & 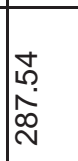 & 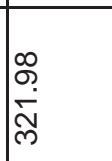 & $\begin{array}{l}\infty \\
\stackrel{\infty}{-} \\
\bar{\sim}\end{array}$ & 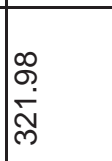 & $\begin{array}{l}\infty \\
\text { ọ } \\
\bar{N} \\
\infty\end{array}$ & 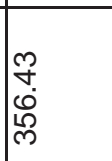 & $\begin{array}{l}\hat{\infty} \\
\dot{\infty} \\
\dot{m}\end{array}$ & 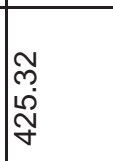 & 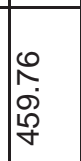 \\
\hline 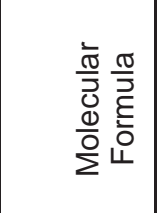 & $\left|\begin{array}{c}N \\
0 \\
0 \\
I \\
N \\
0 \\
0\end{array}\right|$ & $\begin{array}{l}\overline{0} \\
0 \\
0 \\
1 \\
N \\
j\end{array}$ & $\begin{array}{l}\bar{N} \\
0 \\
O_{N} \\
\text { N} \\
0\end{array}$ & 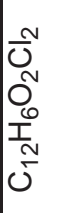 & 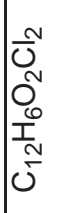 & 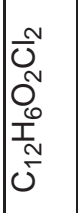 & 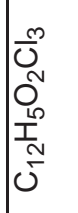 & $\begin{array}{l}0^{+} \\
0_{N} \\
O^{+} \\
I^{N} \\
0\end{array}$ & 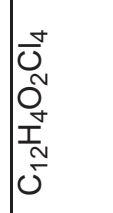 & 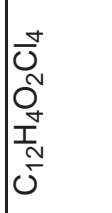 & 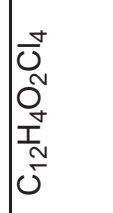 & 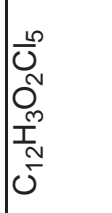 & 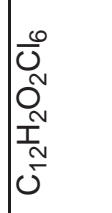 & 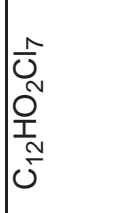 & $\begin{array}{l}\infty \\
0 \\
0 \\
\mathcal{N}^{N} \\
j\end{array}$ \\
\hline $\begin{array}{l}\dot{0} \\
2 \\
0 \\
0\end{array}$ & 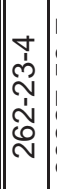 & 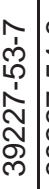 & 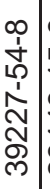 & 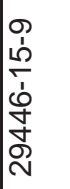 & $\begin{array}{l}0 \\
0 \\
0 \\
N \\
1 \\
1 \\
\infty \\
\infty \\
m\end{array}$ & 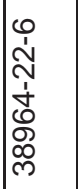 & 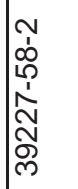 & $\begin{array}{l}\infty \\
0 \\
0 \\
0 \\
1 \\
0 \\
0 \\
1 \\
\hat{0} \\
0 \\
0\end{array}$ & 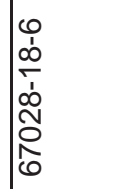 & $\begin{array}{l}\infty \\
0 \\
0 \\
0 \\
1 \\
0 \\
1 \\
0 \\
0 \\
0\end{array}$ & $\begin{array}{l}0 \\
1 \\
0 \\
0 \\
0 \\
⿱ 亠 䒑 \\
\\
\end{array}$ & $\begin{array}{l}\hat{1} \\
\overline{1} \\
\underline{1} \\
\hat{N} \\
\mathcal{N} \\
\tilde{m}\end{array}$ & 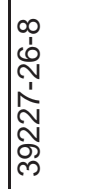 & 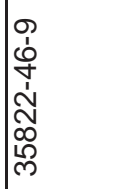 & 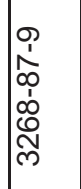 \\
\hline $\begin{array}{l}\overline{\widetilde{J}} \\
\frac{.}{E} \\
\frac{\Phi}{U}\end{array}$ & 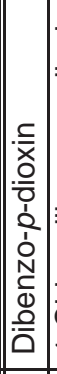 & 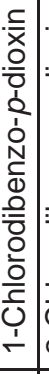 & 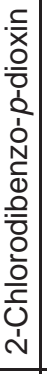 & 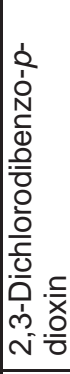 & 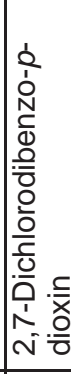 & 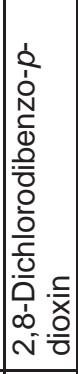 & 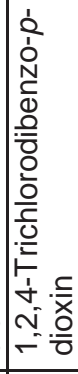 & 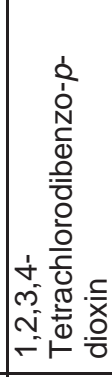 & 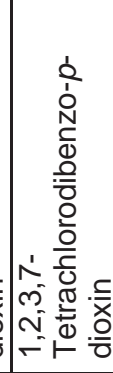 & 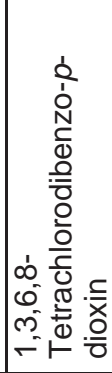 & 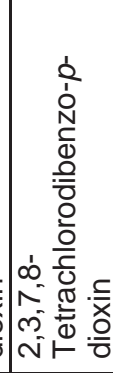 & 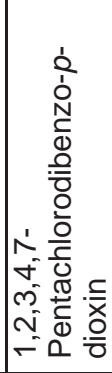 & 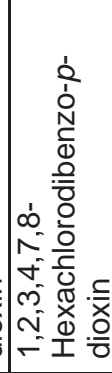 & 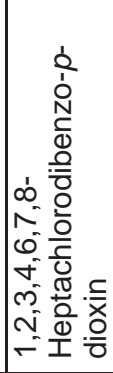 & 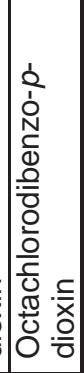 \\
\hline$\dot{z}$ & - & $\sim$ & m) & $\theta$ & ما & 6 & $\Lambda$ & $\infty$ & o & 음 & $\mp$ & $\cong$ & $\stackrel{m}{-1}$ & \pm & $\stackrel{20}{\circ}$ \\
\hline
\end{tabular}

$\frac{a}{u}$ 


\begin{tabular}{|c|c|c|c|c|c|c|c|c|c|}
\hline 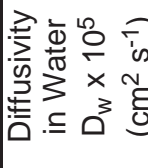 & $\mid \begin{array}{l}N \\
o \\
0 \\
0 \\
0 \\
0\end{array}$ & \begin{tabular}{l}
\multirow{1}{*}{} \\
0 \\
0 \\
0 \\
0
\end{tabular} & 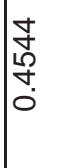 & $\begin{array}{l}\tilde{N} \\
\frac{m}{+} \\
0 \\
0\end{array}$ & $\begin{array}{l}0 \\
\text { N } \\
0 \\
0 \\
0 \\
0\end{array}$ & 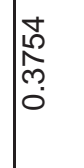 & $\begin{array}{l}0 \\
0 \\
0 \\
\text { m } \\
0\end{array}$ & $\begin{array}{l}0 \\
0 \\
\text { pे } \\
0 \\
0\end{array}$ & 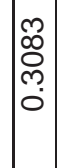 \\
\hline 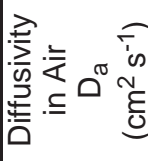 & $\mid$\begin{tabular}{l}
$\infty$ \\
$\infty$ \\
\multirow{1}{0}{} \\
0 \\
0 \\
0
\end{tabular} & $\begin{array}{l}\text { क } \\
8 \\
0 \\
0 \\
0 \\
0\end{array}$ & 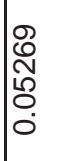 & $\begin{array}{l}\infty \\
0 \\
0 \\
0 \\
0 \\
0\end{array}$ & $\begin{array}{l}0 \\
\infty \\
\infty \\
0 \\
0 \\
0\end{array}$ & $\mid \begin{array}{l}0 \\
\infty \\
\infty \\
0 \\
0 \\
0\end{array}$ & 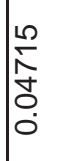 & 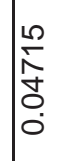 & $\begin{array}{l}0 \\
0 \\
0 \\
0 \\
0 \\
0\end{array}$ \\
\hline 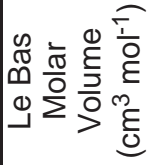 & $\begin{array}{c}\hat{0} \\
\hat{0} \\
\stackrel{2}{2}\end{array}$ & 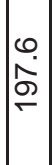 & 梁 & 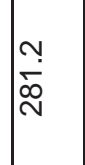 & ন্் & స్తి & o. & $\begin{array}{l}0 \\
\text { ָె } \\
\text { ले }\end{array}$ & 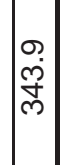 \\
\hline 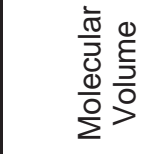 & مُ & 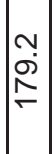 & लि & क्ञ & $\begin{array}{l}0 \\
\stackrel{N}{N} \\
\text { N }\end{array}$ & $\begin{array}{l}0 \\
\text { Na } \\
\text { N }\end{array}$ & হై & হִ & $\begin{array}{l}0 \\
0 \\
0 \\
m\end{array}$ \\
\hline 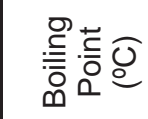 & $\begin{array}{l}0 \\
\text { i. } \\
\text { N } \\
\text { N }\end{array}$ & 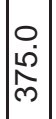 & $\begin{array}{l}m \\
\infty \\
\infty \\
\tilde{\sigma} \\
\end{array}$ & 傗 & 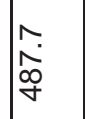 & 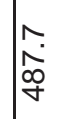 & Nִ & Nִ & $\begin{array}{l}0 \\
\hat{\sim} \\
\hat{n} \\
\end{array}$ \\
\hline 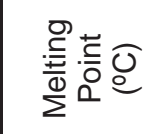 & $\mid \begin{array}{l}\infty \\
\infty \\
\infty\end{array}$ & $\begin{array}{l}0 \\
\dot{\dot{d}} \\
\infty \\
\end{array}$ & 只 & $\begin{array}{l}0 \\
\dot{0} \\
\mathscr{0}\end{array}$ & $\begin{array}{l}\stackrel{\rho}{0} \\
\stackrel{N}{N} \\
\tilde{N}\end{array}$ & 㞷 & $\begin{array}{l}0 \\
0 \\
\tilde{N} \\
\text { N }\end{array}$ & 옴 & 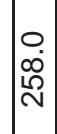 \\
\hline 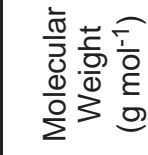 & 足 & 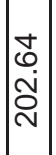 & 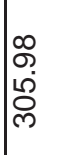 & 䒚 & 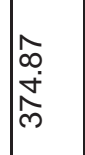 & $\begin{array}{l}\hat{\infty} \\
\infty \\
\dot{N} \\
\hat{m}\end{array}$ & $\begin{array}{l}\bar{m} \\
\ddot{O} \\
\dot{\sigma}\end{array}$ & $\begin{array}{l}\bar{s} \\
\dot{\delta} \\
\dot{\sigma}\end{array}$ & \begin{tabular}{l}
$\stackrel{0}{2}$ \\
$\stackrel{P}{f}$ \\
\multirow{f}{*}{}
\end{tabular} \\
\hline 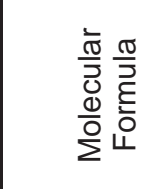 & $\begin{array}{l}0 \\
0^{\infty} \\
I^{2} \\
j \\
j\end{array}$ & 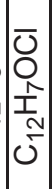 & 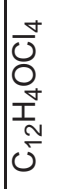 & $\begin{array}{l}\frac{L}{0} \\
0 \\
0 \\
I \\
I \\
j \\
j\end{array}$ & $\begin{array}{l}\frac{0}{0} \\
0 \\
0 \\
N_{N}^{N} \\
0 \\
0\end{array}$ & $\begin{array}{l}0 \\
0 \\
0 \\
0 \\
N_{N}^{N} \\
j\end{array}$ & $\begin{array}{l}\frac{1}{0} \\
0 \\
\frac{1}{1} \\
\tilde{N} \\
j\end{array}$ & $\begin{array}{l}\frac{1}{0} \\
\frac{O}{1} \\
\text { Na } \\
\text { Ũ }\end{array}$ & 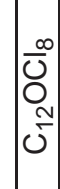 \\
\hline $\begin{array}{l}0 \\
2 \\
0 \\
0\end{array}$ & 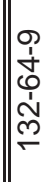 & $\begin{array}{l}0 \\
0 \\
\infty \\
\infty \\
0 \\
0 \\
0 \\
\vdots \\
1\end{array}$ & 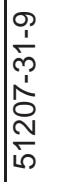 & 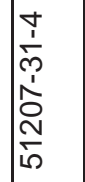 & $\begin{array}{l}0 \\
0 \\
0 \\
N \\
00 \\
0 \\
0 \\
0 \\
0\end{array}$ & 站 & 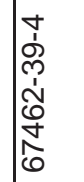 & 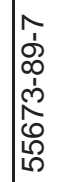 & 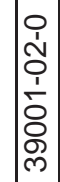 \\
\hline $\begin{array}{l}\overline{\mathscr{J}} \\
\frac{0}{E} \\
\overline{0} \\
\bar{U}\end{array}$ & 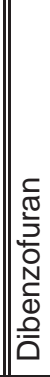 & 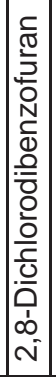 & 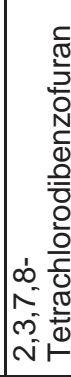 & 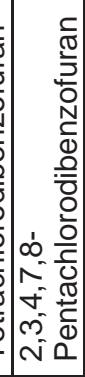 & 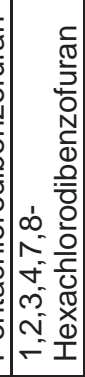 & 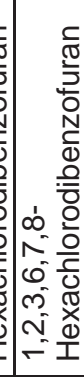 & 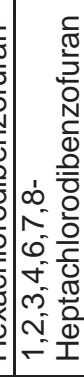 & 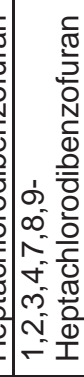 & 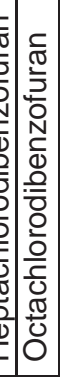 \\
\hline ì & $\|-$ & $\sim$ & & 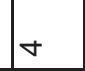 & ما & 6 & $\Lambda$ & $\infty$ & $\sigma$ \\
\hline
\end{tabular}




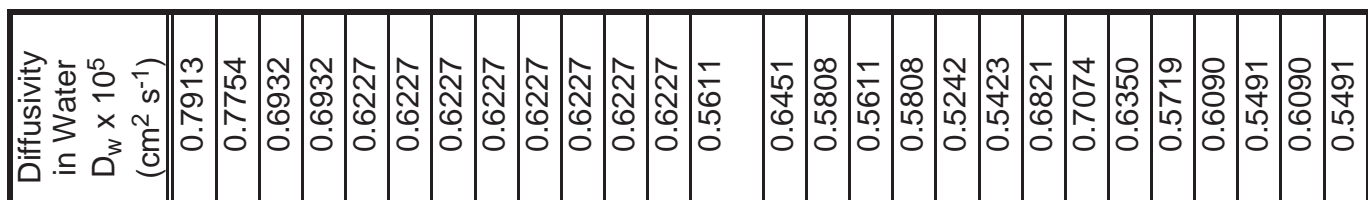

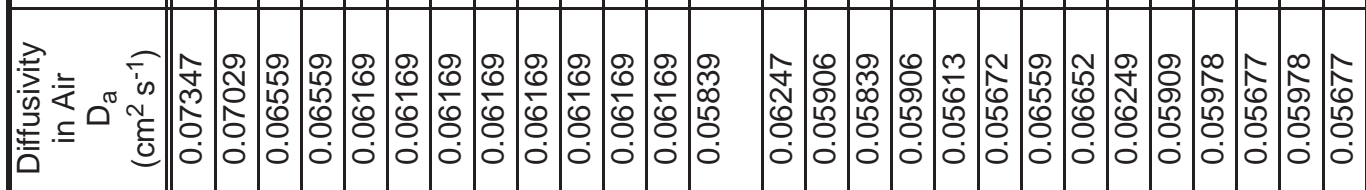

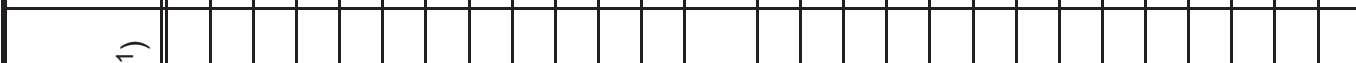

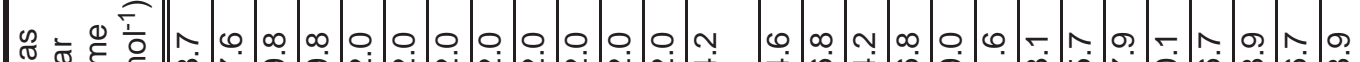

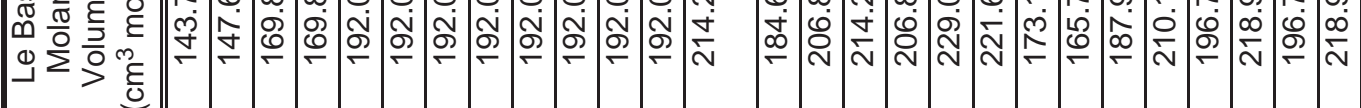

\begin{tabular}{|c|c|c|c|c|c|c|c|c|c|c|c|c|c|c|c|c|c|c|c|c|c|}
\hline 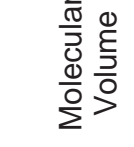 & 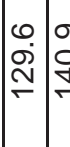 & : & $\frac{8}{6}$ & $\begin{array}{l}\infty \\
\frac{\infty}{\infty} \\
\frac{\infty}{\square}\end{array}$ & 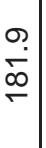 & & $\begin{array}{l}? \\
\vdots \\
0\end{array}$ & ?ִ & 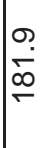 & $\begin{array}{l}\sigma \\
\bar{\infty} \\
\infty \\
\end{array}$ & & $\begin{array}{c}m \\
\stackrel{m}{*} \\
\stackrel{2}{*}\end{array}$ & ?. & & 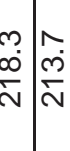 & 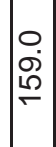 & 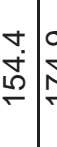 & : & & $\sum$ & $\frac{m}{i}$ \\
\hline 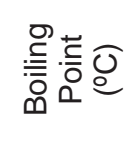 & 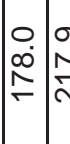 & 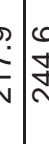 & $\frac{\text { d }}{\frac{d}{d}}$ & $\begin{array}{l}0 \\
\hat{i} \\
\stackrel{0}{N} \\
\end{array}$ & 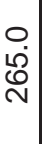 & 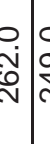 & 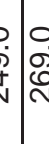 & i & 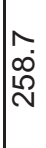 & $\begin{array}{l}\circ \\
\text { Ṅ } \\
\stackrel{2}{N}\end{array}$ & $\begin{array}{l}\text { 이 } \\
\infty \\
\end{array}$ & 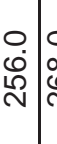 & م) & 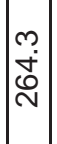 & 일 & $\begin{array}{l}n \\
\stackrel{n}{N} \\
\text { N }\end{array}$ & 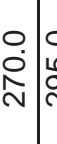 & 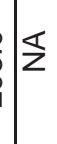 & & & $\begin{array}{l}0 \\
\text { مึ } \\
\text { लె }\end{array}$ \\
\hline 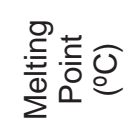 & 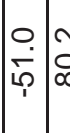 & 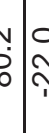 & $\begin{array}{l}0 \\
\dot{m}\end{array}$ & $\frac{0}{\overline{1}}$ & $\stackrel{\pi}{z}$ & r. & مُ & 象 & $\begin{array}{l}\infty \\
\stackrel{\infty}{m} \\
1\end{array}$ & $\begin{array}{l}0 \\
0 \\
P_{1}\end{array}$ & \begin{tabular}{l}
0 \\
\multirow{f}{0}{}
\end{tabular} & $\begin{array}{l}0 \\
\dot{R} \\
\bar{\Lambda}\end{array}$ & 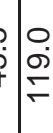 & $\begin{array}{l}0 \\
\stackrel{\leftrightarrow}{\stackrel{2}{ }} \\
\end{array}$ & 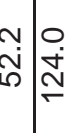 & $\begin{array}{l}\sim \\
\mathscr{8} \\
\mathscr{8}\end{array}$ & & $\mid \begin{array}{l}0 \\
\infty \\
\infty\end{array}$ & & & جִ \\
\hline 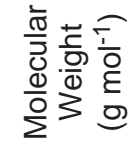 & 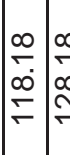 & 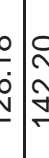 & ป̀ & 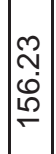 & 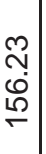 & 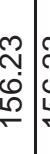 & $\begin{array}{l}3 \\
\vdots \\
\vdots \\
\vdots \\
\end{array}$ & 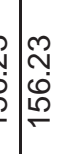 & 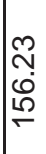 & 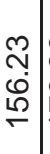 & 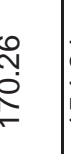 & & 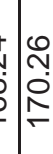 & \begin{tabular}{c}
$\stackrel{+}{N}$ \\
$\infty$ \\
$o$ \\
\hdashline \\
-
\end{tabular} & 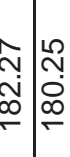 & $\begin{array}{l}\bar{N} \\
\dot{N} \\
\underline{n} \\
\end{array}$ & & 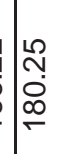 & 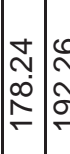 & & 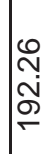 \\
\hline 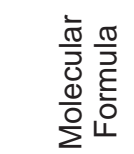 & 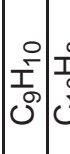 & 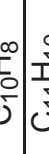 & $\frac{0}{\frac{1}{1}}$ & $=\begin{array}{l}N \\
\\
\tilde{N} \\
0\end{array}$ & $\begin{array}{l}\stackrel{N}{I} \\
N_{0}^{N}\end{array}$ & $\begin{array}{l}\stackrel{N}{T} \\
\tilde{U}^{N}\end{array}$ & 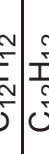 & : va & $\frac{N}{N_{N}^{N}}$ & 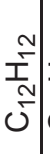 & j) & & 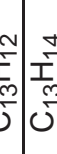 & $\mid \begin{array}{l}\frac{N}{T} \\
\frac{N}{\tilde{m}} \\
\tilde{U}\end{array}$ & & & & $\frac{0}{\frac{1}{4}}$ & & & $\left\{\begin{array}{l}\frac{N}{1} \\
\frac{10}{5}\end{array}\right.$ \\
\hline $\begin{array}{l}\dot{2} \\
\text { co } \\
0\end{array}$ & 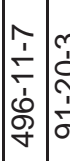 & 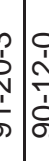 & $\mid \begin{array}{l}0 \\
\frac{1}{10} \\
\frac{1}{1} \\
\frac{1}{a}\end{array}$ & $\begin{array}{l}\infty \\
\infty \\
\infty \\
\infty \\
\infty \\
\infty \\
1 \\
1\end{array}$ & 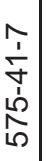 & 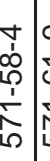 & 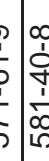 & 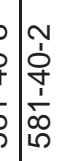 & $\begin{array}{c}0 \\
0 \\
0 \\
N \\
\hat{N} \\
\hat{N} \\
\end{array}$ & 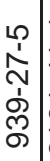 & 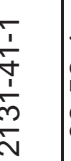 & 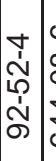 & 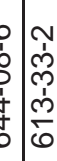 & $\mid \begin{array}{c}1 \\
1 \\
0 \\
0 \\
1 \\
0 \\
\\
\end{array}$ & 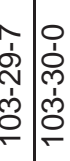 & $\begin{array}{l}\text { D } \\
\grave{N} \\
\tilde{D} \\
\infty \\
\infty\end{array}$ & 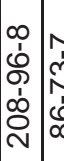 & 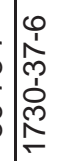 & & 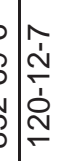 & 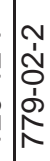 \\
\hline $\begin{array}{l}\overline{\overparen{J}} \\
. \frac{0}{E} \\
\frac{\Phi}{U}\end{array}$ & 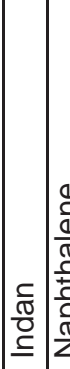 & 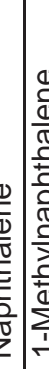 & 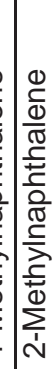 & 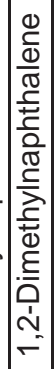 & 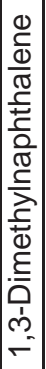 & 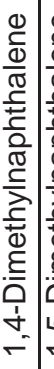 & 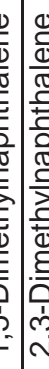 & $\begin{array}{ll}0 \\
\end{array}$ & 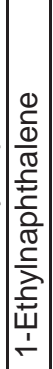 & 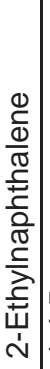 & 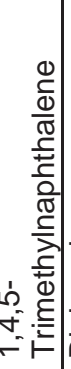 & 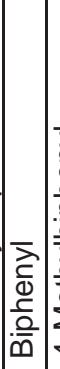 & 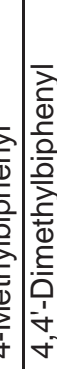 & 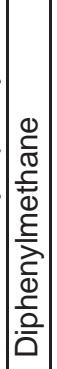 & 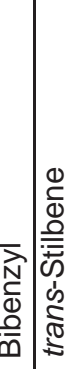 & 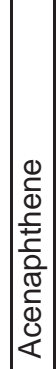 & 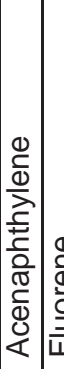 & 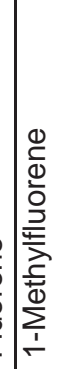 & 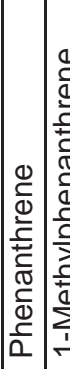 & 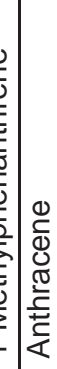 & 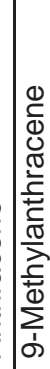 \\
\hline ì & $-\Omega$ & & & ما & 0 & $\wedge$ & \begin{tabular}{l|l}
0 & $\sigma$
\end{tabular} & $1-$ & & - & $\stackrel{m}{\longrightarrow}$ & 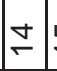 & & 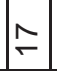 & \begin{tabular}{l|l}
$\infty$ & $\square$
\end{tabular} & న & $\bar{\sim} \mid \AA$ & & & $\stackrel{N}{\stackrel{N}{*}}$ & \\
\hline
\end{tabular}




\begin{tabular}{|c|c|c|c|c|c|c|c|c|c|c|c|c|c|c|c|c|c|c|c|c|c|c|c|c|c|c|}
\hline 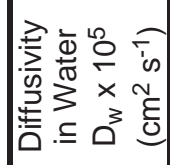 & $\mid \begin{array}{c}\bar{\sigma} \\
\text { ơ } \\
0 \\
0 \\
0\end{array}$ & & 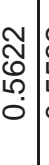 & & 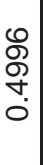 & & $\mid$ & 集 & & 保 & 占 & & 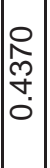 & 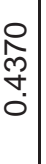 & 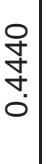 & 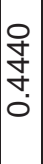 & & $\begin{array}{l}n \\
\infty \\
\infty \\
m \\
0 \\
0\end{array}$ & $\mid \begin{array}{l}n \\
\infty \\
\infty \\
0 \\
0 \\
0\end{array}$ & $\mid \begin{array}{c}\Lambda \\
\infty \\
\infty \\
m \\
0 \\
0\end{array}$ & $\mid \begin{array}{l}1 \\
n \\
n \\
d \\
0 \\
0\end{array}$ & & 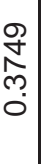 & & & \\
\hline 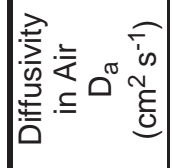 & $\mid \begin{array}{l}\hat{N} \\
\hat{b} \\
0 \\
0 \\
0 \\
0\end{array}$ & 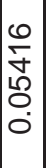 & 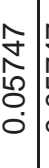 & \begin{tabular}{l|}
$\hat{2}$ \\
$\vdots$ \\
0 \\
0 \\
0 \\
0
\end{tabular} & $\begin{array}{c}\hat{N} \\
\hat{f} \\
0 \\
0 \\
0 \\
0\end{array}$ & $\begin{array}{c}\hat{N} \\
\hat{y} \\
0 \\
0 \\
0 \\
0\end{array}$ & 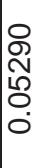 & 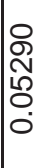 & $\begin{array}{l}\infty \\
0 \\
0 \\
0 \\
0 \\
0 \\
0\end{array}$ & 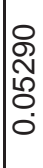 & 㣽 & $\begin{array}{l}0 \\
0 \\
\frac{m}{2} \\
0 \\
0 \\
0\end{array}$ & $\left|\begin{array}{l}0 \\
0 \\
\\
0 \\
0 \\
0\end{array}\right|$ & $\begin{array}{l}0 \\
\frac{1}{2} \\
\frac{1}{0} \\
0 \\
0\end{array}$ & $\begin{array}{l}0 \\
\frac{m}{2} \\
\frac{2}{0} \\
0 \\
0\end{array}$ & $\begin{array}{l}0 \\
m \\
\frac{m}{2} \\
0 \\
0 \\
0\end{array}$ & $\begin{array}{l}0 \\
0 \\
\frac{m}{2} \\
0 \\
0 \\
0\end{array}$ & $\begin{array}{l}\mathscr{m} \\
\infty \\
\infty \\
0 \\
0 \\
0\end{array}$ & $\mid \begin{array}{l}\infty \\
\infty \\
0 \\
0 \\
0 \\
0 \\
0 \\
0\end{array}$ & $\mid \begin{array}{l}\text { N } \\
\mathscr{D} \\
\infty \\
0 \\
0 \\
0 \\
0\end{array}$ & $\mid \begin{array}{l}0 \\
0 \\
0 \\
0 \\
0 \\
0\end{array}$ & 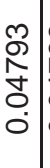 & 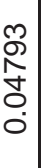 & & & $\begin{array}{l}\tilde{N} \\
\tilde{N} \\
\infty \\
0 \\
0 \\
0\end{array}$ \\
\hline 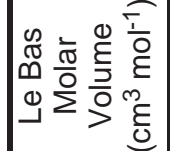 & $\mid \begin{array}{l}\infty \\
\infty \\
\frac{\infty}{N} \\
\sim\end{array}$ & 定 & $\begin{array}{ll}\infty & \\
\dot{m} & \\
\grave{N} & \end{array}$ & & 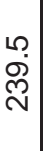 & & & $\begin{array}{l}\infty \\
\stackrel{\infty}{\infty} \\
\text { N }\end{array}$ & N & $\begin{array}{l}\infty \\
\infty \\
\infty \\
\text { N }\end{array}$ & $\begin{array}{l}\infty \\
\infty \\
\stackrel{\leftrightarrow}{\infty}\end{array}$ & 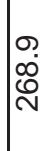 & $\begin{array}{l}O \\
\infty \\
\infty \\
\stackrel{0}{N}\end{array}$ & & & 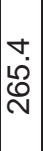 & $\mid \begin{array}{l}+ \\
\dot{d} \\
0 \\
N\end{array}$ & 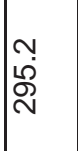 & $\begin{array}{l}\sim \\
\stackrel{2}{\infty} \\
\stackrel{N}{N}\end{array}$ & م & \begin{tabular}{l}
0 \\
\hdashline \\
$\infty$ \\
N
\end{tabular} & 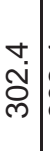 & $\begin{array}{l}\stackrel{+}{\grave{j}} \\
\text { Dे }\end{array}$ & & & $\begin{array}{l}0 \\
\stackrel{\mathcal{D}}{\text { N }}\end{array}$ \\
\hline $\begin{array}{ll}\frac{\bar{\sigma}}{J} & \oplus \\
\bar{\nu} & \frac{E}{5} \\
\frac{\Phi}{0} & \overline{0} \\
\Sigma & =\end{array}$ & $\left(\frac{m}{i}\right.$ & 我 & $\begin{array}{l}m \\
\stackrel{D}{D} \\
N\end{array}$ & 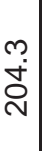 & 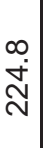 & 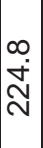 & Na & î́ & 电 & 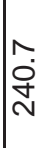 & No & $\begin{array}{l}\text { N } \\
\stackrel{5}{N}\end{array}$ & 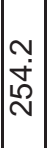 & 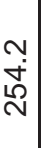 & 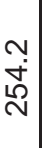 & 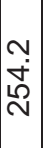 & 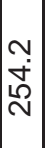 & $\frac{\infty}{\infty}$ & $\mid \infty$ & 范 & - & $\begin{array}{l}0 \\
\dot{0} \\
\text { N }\end{array}$ & $\begin{array}{l}0 \\
\dot{Q} \\
\stackrel{\mathrm{N}}{\mathbf{N}}\end{array}$ & & & 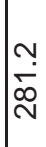 \\
\hline 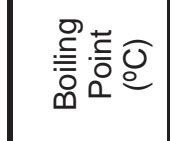 & \begin{tabular}{|l}
0 \\
$\infty$ \\
$\infty$ \\
$\infty$
\end{tabular} & 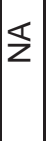 & $\begin{array}{l}0 \\
\dot{q} \\
\dot{q}\end{array}$ & 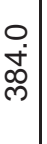 & $\begin{array}{l}0 \\
\dot{\rho} \\
\stackrel{q}{q}\end{array}$ & $\begin{array}{l}0 \\
\text { ì } \\
\text { ơ }\end{array}$ & 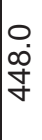 & 品 & O्. & 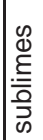 & $\begin{array}{l}\mathscr{\varphi} \\
\hat{m} \\
\dot{q}\end{array}$ & 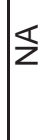 & 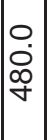 & $\begin{array}{l}0 \\
\dot{0} \\
\dot{0} \\
+\end{array}$ & 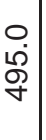 & 畺 & $\begin{array}{l}0 \\
\dot{\partial} \\
\dot{g}\end{array}$ & $\varangle$ & 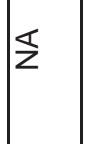 & 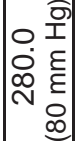 & & & $\begin{array}{l}0 \\
\infty \\
\infty \\
\infty\end{array}$ & 芯 & & 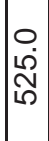 \\
\hline 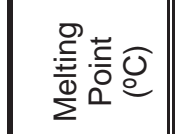 & $\begin{array}{l}0 \\
\text { Oे } \\
\text { N }\end{array}$ & $\begin{array}{l}0 \\
\stackrel{2}{i} \\
\infty\end{array}$ & $\frac{N}{5}$ & $\begin{array}{l}\infty \\
\text { i. } \\
0 \\
-\end{array}$ & \begin{tabular}{l}
0 \\
$\stackrel{0}{\infty}$ \\
$\infty$ \\
\hdashline \\
\end{tabular} & $\begin{array}{l}0 \\
\stackrel{i}{N} \\
\stackrel{N}{N}\end{array}$ & 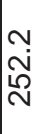 & $\begin{array}{l}\circ \\
\stackrel{8}{\circ} \\
\end{array}$ & $\frac{O}{\stackrel{m}{N}}$ & 只. & $\begin{array}{l}0 \\
\infty \\
0 \\
0 \\
\end{array}$ & 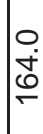 & \begin{tabular}{|l|}
0 \\
0 \\
0 \\
0 \\
\end{tabular} & $\begin{array}{l}0 \\
\stackrel{1}{N} \\
\end{array}$ & 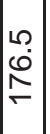 & مِ & $\begin{array}{l}0 \\
\dot{N} \\
\text { N }\end{array}$ & 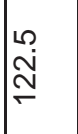 & $\begin{array}{l}\stackrel{0}{\mathrm{~N}} \\
\stackrel{\mathrm{N}}{\mathrm{N}}\end{array}$ & 官 & $\mid \begin{array}{l}0 \\
\infty \\
D \\
\\
\end{array}$ & & $\begin{array}{l}0 \\
\dot{0} \\
\stackrel{N}{ }\end{array}$ & & & 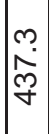 \\
\hline 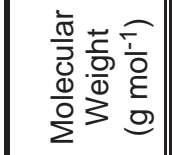 & 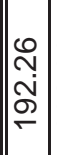 & $\begin{array}{l}\text { N } \\
\text { @ } \\
D \\
\text { N }\end{array}$ & 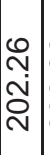 & $\mid \begin{array}{c}\stackrel{0}{N} \\
\mathbb{N} \\
\stackrel{N}{N}\end{array}$ & 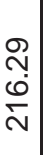 & 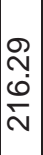 & 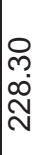 & 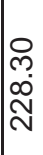 & $\begin{array}{l}\bar{m} \\
\stackrel{\infty}{0} \\
\stackrel{\infty}{N}\end{array}$ & $\begin{array}{l}\stackrel{\infty}{\infty} \\
\infty \\
\stackrel{N}{N} \\
\text { Na }\end{array}$ & $\begin{array}{l}\stackrel{\rho}{\infty} \\
\stackrel{\text { N }}{\text { N̦ }}\end{array}$ & $\begin{array}{l}\tilde{N} \\
\text { స్ట } \\
\text { Nె }\end{array}$ & 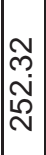 & 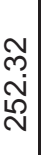 & 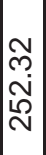 & 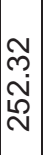 & 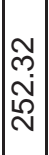 & 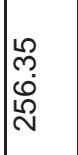 & 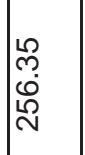 & 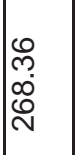 & $\begin{array}{l}0 \\
m \\
\infty \\
\varrho \\
N\end{array}$ & 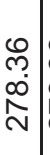 & 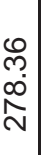 & $\begin{array}{l}\text { co } \\
\text { ले } \\
\text { aे } \\
\text { N }\end{array}$ & & | \\
\hline 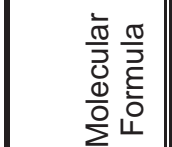 & 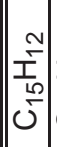 & 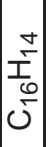 & $\begin{array}{c}\stackrel{0}{1} \\
\frac{1}{1} \\
\dot{0} \\
j\end{array}$ & $\begin{array}{l}\frac{0}{1} \\
\frac{7}{6} \\
j\end{array}$ & $\begin{array}{l}\stackrel{N}{T} \\
\frac{N}{0} \\
0\end{array}$ & 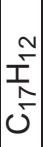 & $\begin{array}{l}\stackrel{N}{I} \\
\frac{N}{\infty} \\
j\end{array}$ & 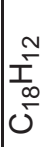 & $\begin{array}{l}\stackrel{+}{+} \\
\frac{1}{\infty} \\
j\end{array}$ & 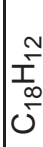 & $\frac{\widetilde{N}}{N_{\infty}^{\infty}}$ & $\frac{\widetilde{T}}{\bar{I}}$ & & 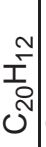 & 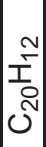 & 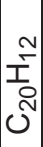 & 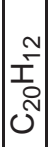 & 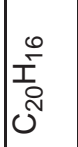 & 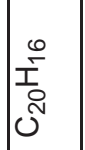 & $\begin{array}{l}\frac{0}{1} \\
\stackrel{+}{N} \\
\hat{N}\end{array}$ & $\left|\begin{array}{c}0 \\
\frac{0}{I} \\
\bar{N} \\
\mathcal{N}\end{array}\right|$ & $\begin{array}{l}\stackrel{\nabla}{ \pm} \\
\underset{N}{N} \\
J^{2}\end{array}$ & $\begin{array}{l}\stackrel{+}{ \pm} \\
\text { N } \\
\tilde{N}^{\prime}\end{array}$ & $\tilde{N}^{N}$ & & $\frac{\substack{N \\
\text { N }}}{\tilde{N}}$ \\
\hline 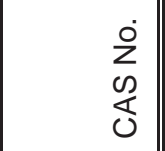 & 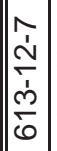 & 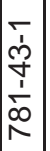 & $\begin{array}{l}0 \\
0 \\
0 \\
0 \\
1 \\
\text { ஸे } \\
-1\end{array}$ & 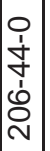 & $\begin{array}{l}0 \\
1 \\
1 \\
o \\
0 \\
0 \\
\tilde{N}\end{array}$ & 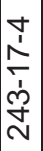 & $\mid \begin{array}{l}0 \\
1 \\
0 \\
0 \\
0 \\
\frac{1}{N}\end{array}$ & 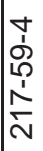 & $\begin{array}{l}+ \\
\dot{y} \\
\text { के } \\
\dot{1} \\
\text { के }\end{array}$ & 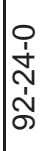 & $\begin{array}{l}m \\
1 \\
1 \\
1 \\
0 \\
0 \\
1\end{array}$ & 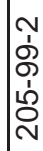 & 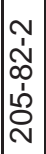 & $\begin{array}{l}0 \\
0 \\
0 \\
0 \\
1 \\
\\
\end{array}$ & $\begin{array}{c}\infty \\
0 \\
i \\
\tilde{w} \\
0 \\
0 \\
i \\
1\end{array}$ & 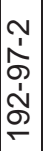 & 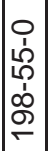 & $\begin{array}{l}0 \\
1 \\
1 \\
0 \\
1 \\
1 \\
1\end{array}$ & \begin{tabular}{|l}
0 \\
0 \\
0 \\
0 \\
0 \\
0 \\
10
\end{tabular} & | & 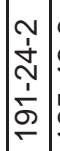 & 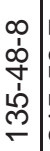 & 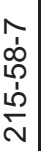 & $\begin{array}{l}e^{\infty} \\
\stackrel{c}{c} \\
c\end{array}$ & & 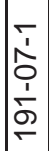 \\
\hline 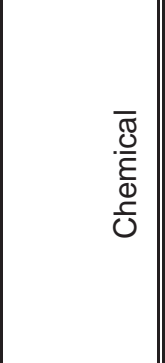 & 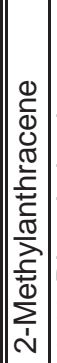 & 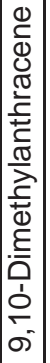 & 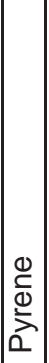 & 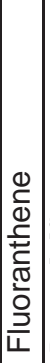 & 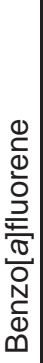 & 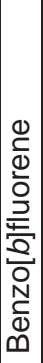 & 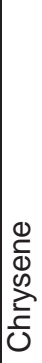 & 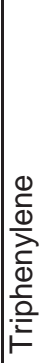 & 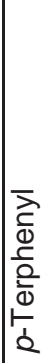 & 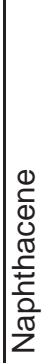 & 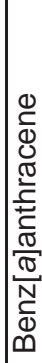 & 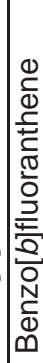 & 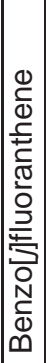 & 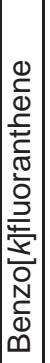 & 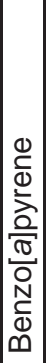 & 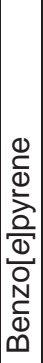 & 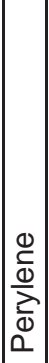 & 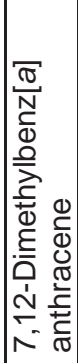 & 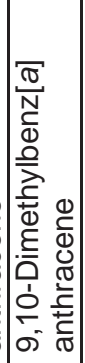 & 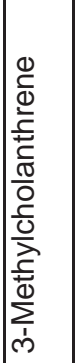 & 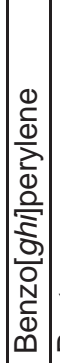 & $\begin{array}{l} \\
\\
\mathbb{2} \\
\\
0 \\
0 \\
\frac{\pi}{2} \\
0 \\
0\end{array}$ & 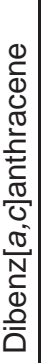 & & & 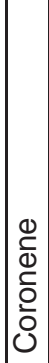 \\
\hline$\dot{z}$ & $\stackrel{\infty}{\sim}$ & $\mathscr{N}$ & లি & $\bar{m}$ & స్ & ભ & ले & Lొ & 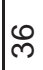 & $\hat{m}$ & $\stackrel{\infty}{\infty}$ & $m$ & 우 & 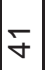 & F & 守 & 18 & L & 果 & $\hat{\forall}$ & $\underset{\sim}{\infty}$ & 守 & 잉 & 6 & & బొ \\
\hline
\end{tabular}




\begin{tabular}{|c|c|c|c|c|c|c|c|c|c|c|c|c|c|c|c|c|c|c|c|c|c|c|c|c|}
\hline 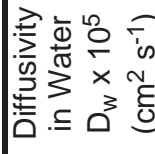 & $\mid \begin{array}{l}N \\
0 \\
0 \\
\infty \\
0 \\
0 \\
0\end{array}$ & $\frac{1}{0}$ & $\begin{array}{l}\qquad \\
\infty \\
\frac{1}{N} \\
0\end{array}$ & $\left|\begin{array}{l}0 \\
\infty \\
T \\
0 \\
0\end{array}\right|$ & & 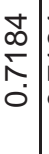 & & \begin{tabular}{c}
$\frac{1}{\infty}$ \\
\multirow{\infty}{*}{} \\
0
\end{tabular} & & 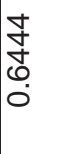 & 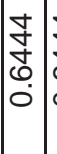 & 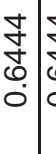 & $\begin{array}{l}\dot{y} \\
\dot{f} \\
\dot{0} \\
\dot{0}\end{array}$ & 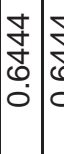 & 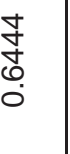 & $\mid \begin{array}{l}8 \\
8 \\
0 \\
0\end{array}$ & 告 & $\mid \begin{array}{c}0 \\
0 \\
\infty \\
0 \\
0 \\
0\end{array}$ & & 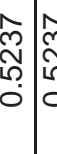 & & 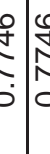 & & 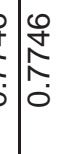 \\
\hline 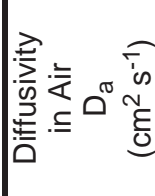 & $\mid \begin{array}{l}9 \\
0 \\
0 \\
0 \\
0 \\
0 \\
0\end{array}$ & $\begin{array}{l}\mathscr{D} \\
\mathbb{\infty} \\
0 \\
0 \\
0 \\
0\end{array}$ & $\begin{array}{l}\mathscr{D} \\
\mathbb{\infty} \\
0 \\
0 \\
0 \\
0\end{array}$ & 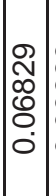 & 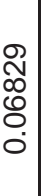 & 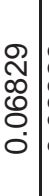 & $\begin{array}{l}\text { Dे } \\
0 \\
0 \\
0 \\
0\end{array}$ & $\begin{array}{l}\mathscr{9} \\
0 \\
0 \\
0 \\
\vdots \\
0\end{array}$ & 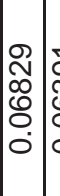 & $\begin{array}{l}\overline{0} \\
\bar{్} \\
0 \\
0 \\
0\end{array}$ & $\mid \begin{array}{l}\bar{D} \\
\bar{ల} \\
0 \\
0 \\
0\end{array}$ & 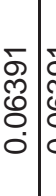 & 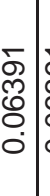 & \begin{tabular}{l|l}
- & \\
& 0 \\
0 & 0 \\
0 & 0 \\
0 & 0 \\
0 & 0
\end{tabular} & 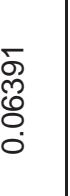 & $\mid \begin{array}{l}\bar{乃} \\
\text { ర్ల } \\
0 \\
0\end{array}$ & $\begin{array}{l}\bar{D} \\
\bar{ల} \\
0 \\
0 \\
0\end{array}$ & 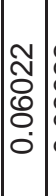 & 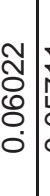 & 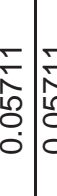 & & ב. & & i. \\
\hline 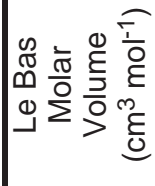 & 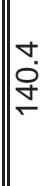 & $\begin{array}{l}0 \\
\stackrel{0}{0} \\
\underline{\sigma}\end{array}$ & 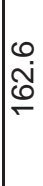 & 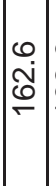 & $\begin{array}{l}0 \\
\stackrel{0}{ن} \\
\stackrel{0}{ }\end{array}$ & 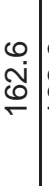 & $\begin{array}{l}0 \\
\stackrel{0}{ن} \\
\underline{\varrho}\end{array}$ & & 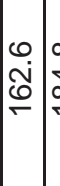 & $\begin{array}{l}\infty \\
\dot{\infty} \\
\infty \\
-\end{array}$ & $\begin{array}{l}\infty \\
\infty \\
\dot{\infty} \\
\infty \\
\end{array}$ & \begin{tabular}{l}
$\infty$ \\
$\dot{0}$ \\
$\substack{+ \\
\hdashline}$
\end{tabular} & $\begin{array}{l}\infty \\
\dot{\infty} \\
\dot{\infty} \\
\stackrel{+}{*}\end{array}$ & 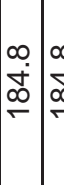 & 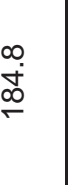 & 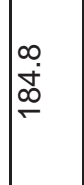 & $\begin{array}{l}\infty \\
\dot{x} \\
\stackrel{\infty}{+}\end{array}$ & $\begin{array}{c}0 \\
\stackrel{0}{0} \\
\text { N }\end{array}$ & 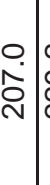 & 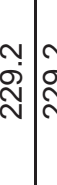 & & & & 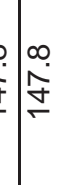 \\
\hline 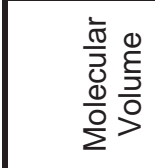 & Oె & م & 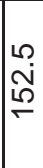 & 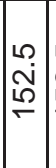 & مِ & 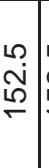 & 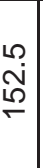 & مِ & 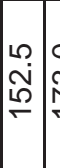 & مִ & 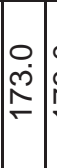 & 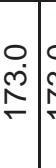 & $\begin{array}{l}0 \\
\\
\\
\end{array}$ & 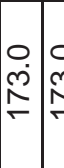 & ֻ̊ & مُ & $\begin{array}{l}0 \\
\stackrel{0}{N} \\
\stackrel{N}{c}\end{array}$ & 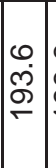 & 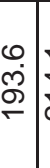 & $\underset{\dot{J}}{\stackrel{r}{N}}$ & & & & $\stackrel{-}{-\infty}$ \\
\hline 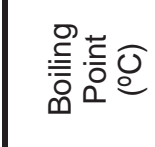 & 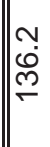 & 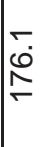 & ه্. & $\begin{array}{c}r \\
\dot{j} \\
\underline{\sigma}\end{array}$ & 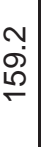 & \begin{tabular}{l}
\multirow{2}{*}{} \\
$\dot{v}$ \\
$\stackrel{2}{-}$
\end{tabular} & 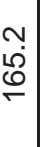 & | & 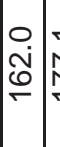 & $\stackrel{\Sigma}{\Sigma}$ & $\begin{array}{ll}0 \\
\ddot{\infty} \\
\infty \\
\infty\end{array}$ & 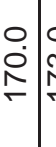 & $\begin{array}{l}0 \\
\\
\\
\end{array}$ & 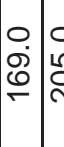 & مْ & $\begin{array}{l}0 \\
\infty \\
\infty \\
0 \\
\end{array}$ & $\begin{array}{l}\infty \\
\stackrel{\infty}{0} \\
\stackrel{0}{-}\end{array}$ & 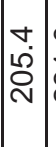 & $\begin{array}{l}0 \\
\overline{-} \\
\bar{N} \\
\end{array}$ & 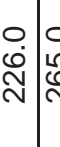 & & & & j. \\
\hline 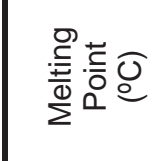 & 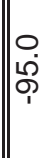 & & 䎹 & 帘 & $\mid \begin{array}{c}0 \\
\\
0 \\
\\
1\end{array}$ & $\begin{array}{l}0 \\
0 \\
\dot{O} \\
1\end{array}$ & $\begin{array}{l}\infty \\
\dot{0} \\
\infty \\
1\end{array}$ & 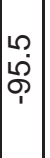 & 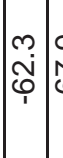 & 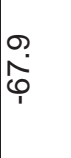 & $\mid \begin{array}{l}0 \\
\infty \\
\infty \\
\infty \\
1 \\
1\end{array}$ & $\frac{0}{\vdots}$ & 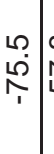 & 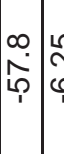 & 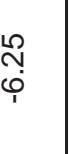 & 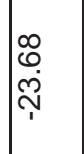 & 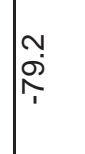 & 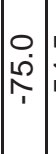 & & & & & & 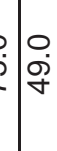 \\
\hline 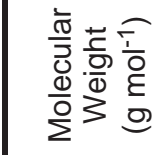 & 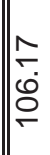 & 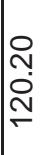 & 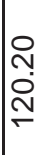 & 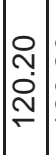 & $\begin{array}{l}\stackrel{N}{N} \\
\stackrel{N}{1} \\
\end{array}$ & $\begin{array}{l}\stackrel{N}{N} \\
\stackrel{N}{0} \\
\end{array}$ & 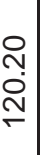 & 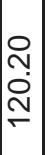 & 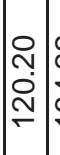 & 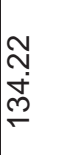 & 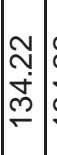 & 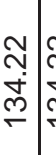 & 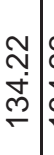 & 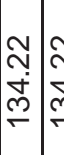 & $\begin{array}{l}\text { Ñ } \\
\text { ले } \\
\end{array}$ & $\begin{array}{l}\tilde{N} \\
\stackrel{N}{+} \\
\\
\end{array}$ & 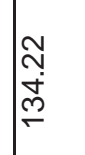 & 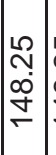 & 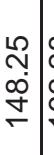 & 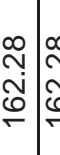 & 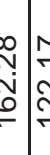 & N & & 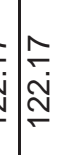 \\
\hline 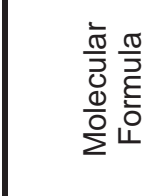 & $\mid \begin{array}{l}\stackrel{0}{1} \\
T_{0}^{\infty} \\
0\end{array}$ & 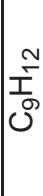 & $\frac{N}{\mathcal{N}}$ & $\begin{array}{c}\stackrel{N}{T} \\
\stackrel{N}{0} \\
\mathcal{O}\end{array}$ & $\begin{array}{l}\stackrel{N}{I} \\
\stackrel{0}{0} \\
0\end{array}$ & $\begin{array}{c}\stackrel{N}{T} \\
\stackrel{0}{0} \\
0\end{array}$ & & $\begin{array}{l}\stackrel{N}{1} \\
\stackrel{0}{0} \\
0\end{array}$ & 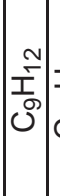 & $\begin{array}{l}\frac{+}{1} \\
\frac{1}{0} \\
j\end{array}$ & $\mid \begin{array}{l}+ \\
⿱ 亠 䒑 \\
0 \\
0 \\
0\end{array}$ & 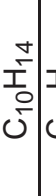 & $\begin{array}{l}+ \\
\vdots \\
\vdots \\
0 \\
0\end{array}$ & \begin{tabular}{l|l} 
\\
$\vdots$ \\
\\
0 \\
$ن$
\end{tabular} & $\begin{array}{l}\frac{j}{1} \\
\text { jo }\end{array}$ & 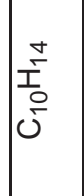 & 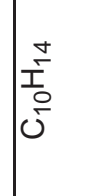 & & & & & & & 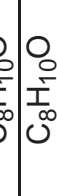 \\
\hline $\begin{array}{l}\dot{2} \\
0 \\
0 \\
0\end{array}$ & $\mid \begin{array}{l}\dot{y} \\
\dot{y} \\
\dot{y} \\
0 \\
0 \\
0\end{array}$ & 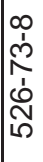 & 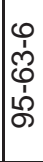 & $\mid \begin{array}{l}\infty \\
1 \\
1 \\
0 \\
0 \\
0 \\
0 \\
-1\end{array}$ & $\begin{array}{l}\overline{1} \\
1 \\
0 \\
0 \\
1 \\
0 \\
0\end{array}$ & $\begin{array}{l}\infty \\
\infty \\
\sim \\
\infty \\
\infty \\
\infty \\
\infty \\
\sigma\end{array}$ & $\begin{array}{c}\frac{0}{1} \\
+ \\
\frac{1}{1} \\
\frac{1}{6}\end{array}$ & 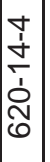 & $\left|\begin{array}{l}\infty \\
0 \\
0 \\
0 \\
\infty \\
\dot{N} \\
0 \\
0\end{array}\right|$ & \begin{tabular}{l}
0 \\
1 \\
1 \\
0 \\
1 \\
$\dot{1}$ \\
\multicolumn{1}{c}{}
\end{tabular} & 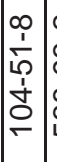 & 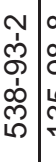 & 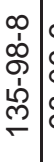 & 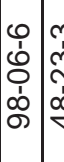 & $\begin{array}{l}\text { m } \\
\text { ले } \\
\text { ó } \\
\text { + }\end{array}$ & $\begin{array}{l}\hat{N} \\
\hat{n} \\
\hat{n} \\
\hat{N} \\
\hat{N}\end{array}$ & 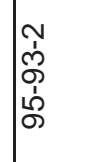 & 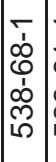 & 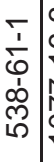 & 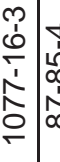 & 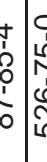 & 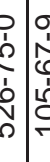 & & 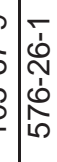 \\
\hline $\begin{array}{l}\overline{\widetilde{J}} \\
. \frac{\mathcal{E}}{E} \\
\bar{\Phi} \\
\bar{U}\end{array}$ & 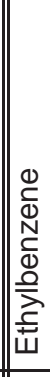 & 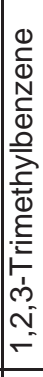 & 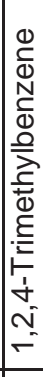 & 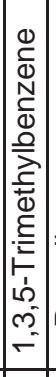 & 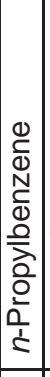 & 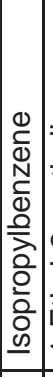 & 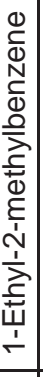 & 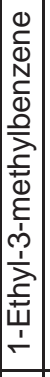 & 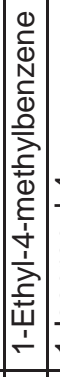 & 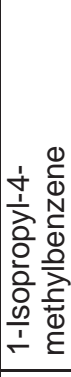 & 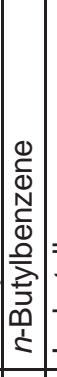 & 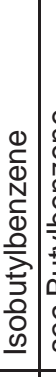 & 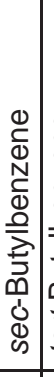 & 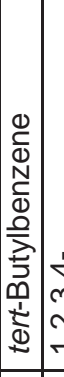 & 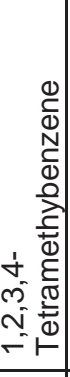 & 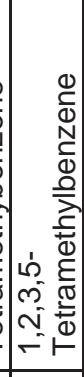 & 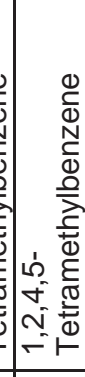 & 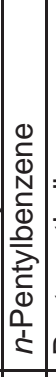 & 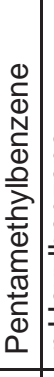 & 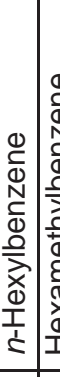 & 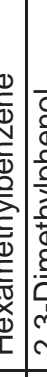 & 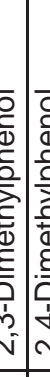 & & 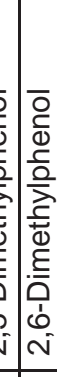 \\
\hline ì & & $\sim$ & m & ナ & 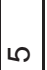 & 0 & T & $\infty$ & க) & 으 & $=$ & $\div$ & $m$ & 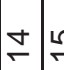 & $\stackrel{2}{\square}$ & $\stackrel{\oplus}{\circ}$ & $\stackrel{1}{-}$ & $\infty$ & 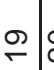 & ㅇ. & 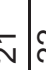 & N & & $\stackrel{N}{N}$ \\
\hline
\end{tabular}




\begin{tabular}{|c|c|c|c|c|c|c|c|c|c|c|c|c|c|c|c|c|c|c|c|c|c|c|c|c|c|}
\hline 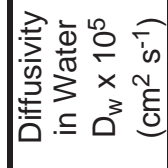 & $\mid \begin{array}{c}0 \\
N \\
N \\
0 \\
0\end{array}$ & 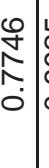 & 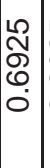 & 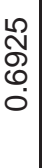 & $\mid$ & $\mid$ & $\mid$ & م્ّ & 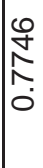 & $\mid \begin{array}{l}0 \\
0 \\
N \\
0 \\
0 \\
0\end{array}$ & 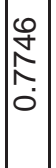 & 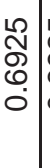 & $\begin{array}{l}\stackrel{2}{2} \\
\text { o. } \\
0 \\
0\end{array}$ & 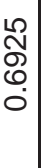 & 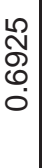 & \begin{tabular}{|}
$\bar{N}$ \\
$\tilde{N}$ \\
0 \\
0 \\
0
\end{tabular} & 芯 & $\begin{array}{c}\bar{N} \\
\mathbb{N} \\
0 \\
0\end{array}$ & & & $\begin{array}{c}\bar{N} \\
\text { c్ } \\
0\end{array}$ & $\begin{array}{l}0 \\
0 \\
0 \\
10 \\
0 \\
0\end{array}$ & & $\stackrel{\leftrightarrow}{\circ}$ & 歺 \\
\hline 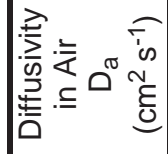 & 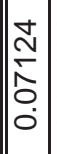 & 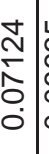 & $\begin{array}{l}1 \\
\dddot{2} \\
0 \\
0 \\
0 \\
0\end{array}$ & $\begin{array}{l}1 \\
0 \\
0 \\
0 \\
0 \\
0\end{array}$ & $\begin{array}{l}1 \\
0 \\
0 \\
0 \\
0 \\
0\end{array}$ & $\begin{array}{l}0 \\
0 \\
0 \\
0 \\
0 \\
0\end{array}$ & $\mid \begin{array}{l}n \\
0 \\
0 \\
0 \\
0 \\
0\end{array}$ & $\begin{array}{l}\text { L } \\
\text { ర్ } \\
\stackrel{0}{0} \\
0 \\
0\end{array}$ & 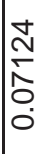 & 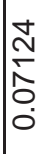 & \begin{tabular}{c}
\multirow{2}{*}{} \\
$\stackrel{1}{1}$ \\
$\mathbf{O}$ \\
0 \\
0
\end{tabular} & 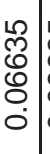 & $\begin{array}{l}1 \\
\stackrel{0}{0} \\
0 \\
0 \\
0\end{array}$ & $\begin{array}{l}1 \\
0 \\
0 \\
0 \\
0 \\
0\end{array}$ & $\begin{array}{l}0 \\
\dddot{0} \\
0 \\
0 \\
0 \\
0\end{array}$ & 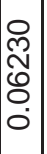 & 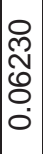 & 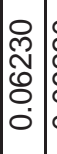 & 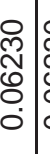 & 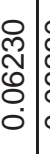 & $\begin{array}{c}0 \\
\text { స్ } \\
0 \\
0 \\
0\end{array}$ & $\begin{array}{l}\bar{S} \\
\infty \\
0 \\
0 \\
0 \\
0\end{array}$ & & \begin{tabular}{l}
\multirow{N}{N}{} \\
$\frac{1}{0}$ \\
0 \\
0
\end{tabular} & $\begin{array}{l}\text { N } \\
\text { के } \\
\text { Oุ } \\
0\end{array}$ \\
\hline 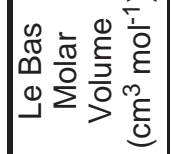 & $\mid$\begin{tabular}{c}
$\infty$ \\
\multirow{2}{f}{} \\
+ \\
\end{tabular} & $\begin{array}{l}\infty \\
\dot{\sim} \\
\dot{y} \\
+\end{array}$ & $\begin{array}{l}0 \\
0 \\
0 \\
-1\end{array}$ & $\begin{array}{l}0 \\
\dot{0} \\
\vdots \\
\end{array}$ & $\begin{array}{l}0 \\
\vdots \\
\stackrel{0}{0} \\
\end{array}$ & $\begin{array}{l}0 \\
0 \\
\dot{2} \\
\end{array}$ & $\begin{array}{l}0 \\
0 \\
0 \\
0\end{array}$ & 足 & $\begin{array}{l}\infty \\
\stackrel{\sim}{+} \\
\leftarrow\end{array}$ & $\begin{array}{l}\infty \\
\stackrel{\sim}{+} \\
\end{array}$ & \begin{tabular}{l}
$\infty$ \\
\multirow{N}{f}{} \\
- \\
\end{tabular} & $\begin{array}{l}0 \\
\stackrel{0}{R} \\
\stackrel{2}{-}\end{array}$ & $\begin{array}{l}0 \\
\dot{0} \\
\stackrel{2}{2}\end{array}$ & $\begin{array}{l}0 \\
\vdots \\
\stackrel{0}{2}\end{array}$ & $\begin{array}{l}0 \\
\stackrel{0}{2} \\
\end{array}$ & 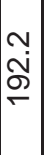 & 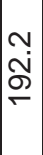 & & 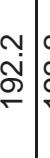 & 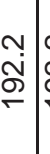 & 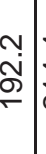 & 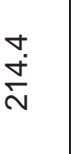 & & & 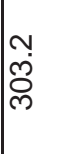 \\
\hline 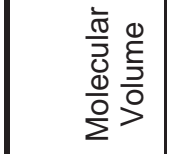 & $\mid \begin{array}{c}- \\
\infty \\
\infty \\
c \\
-\infty\end{array}$ & $\begin{array}{c}- \\
\infty \\
m \\
-\end{array}$ & $\begin{array}{l}0 \\
\infty \\
\infty \\
0 \\
\end{array}$ & 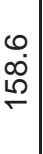 & $\begin{array}{l}0 \\
0 \\
0 \\
\stackrel{0}{0} \\
\end{array}$ & $\begin{array}{l}0 \\
0 \\
0 \\
\stackrel{\rho}{0} \\
\end{array}$ & $\mid \begin{array}{c}0 \\
0 \\
0 \\
\stackrel{0}{0} \\
\end{array}$ & $\begin{array}{l}0 \\
\infty \\
\stackrel{\infty}{0} \\
\stackrel{0}{0}\end{array}$ & ci & & $\begin{array}{l}- \\
\infty \\
m \\
-\infty\end{array}$ & $\begin{array}{c}0 \\
\infty \\
\infty \\
0 \\
-1 \\
\end{array}$ & $\mid \begin{array}{l}0 \\
\infty \\
\infty \\
0 \\
\end{array}$ & $\mid \begin{array}{l}0 \\
\infty \\
\infty \\
\infty \\
-1\end{array}$ & $\begin{array}{l}0 \\
0 \\
\infty \\
\stackrel{0}{0} \\
\end{array}$ & 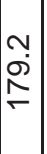 & 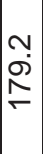 & 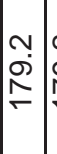 & 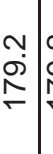 & 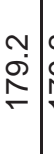 & 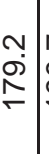 & & & $\frac{N}{\bar{c}}$ & 我 \\
\hline 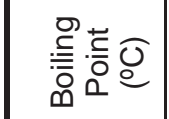 & o & 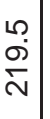 & 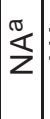 & $\Sigma$ & $\mathbb{z}$ & $\mathbb{z}$ & $\$$ & 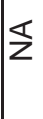 & 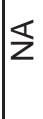 & $\mathbb{z}$ & $\Sigma$ & $\varangle$ & 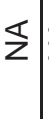 & 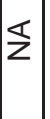 & $\mathbb{z}$ & $\mathbb{z}$ & $\varangle$ & $\varangle$ & $\Sigma$ & \begin{tabular}{l|l}
0 \\
$\dot{\sigma}$ \\
$\dot{d}$
\end{tabular} & 吕 & $\mathbb{z}$ & 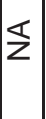 & 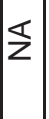 & 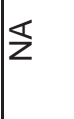 \\
\hline 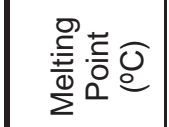 & $\mid \begin{array}{l}0 \\
i \\
0 \\
0\end{array}$ & $\begin{array}{l}0 \\
\infty \\
0\end{array}$ & $\begin{array}{l}0 \\
\mathscr{M}\end{array}$ & $\begin{array}{l}0 \\
\dot{c} \\
\end{array}$ & $\begin{array}{l}0 \\
\mathfrak{N} \\
\end{array}$ & 웃 & $\mid \begin{array}{l}0 \\
\infty \\
0 \\
0\end{array}$ & $\$$ & $\varangle$ & 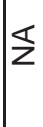 & $\begin{array}{l}0 \\
\dot{\forall}\end{array}$ & 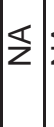 & $\mathbb{z}$ & 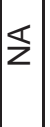 & $\begin{array}{l}0 \\
\dot{0} \\
8\end{array}$ & $\mathbb{z}$ & 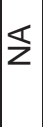 & & $\frac{0}{\dot{\gamma}}$ & $\begin{array}{l}0 \\
\bar{\sigma}\end{array}$ & $\begin{array}{l}\circ \\
\dot{8}\end{array}$ & $\mathbb{z}$ & & $\varangle$ & $\frac{0}{i}$ \\
\hline 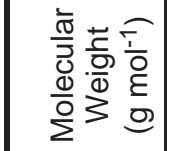 & $\mid \begin{array}{c}\hat{N} \\
\stackrel{\mathrm{N}}{\mathrm{N}} \\
-\end{array}$ & $\begin{array}{l}\stackrel{N}{\check{N}} \\
\stackrel{N}{-}\end{array}$ & $\begin{array}{l}\text { N } \\
0 \\
0 \\
- \\
-\end{array}$ & 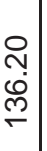 & 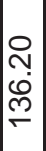 & 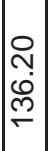 & $\begin{array}{l}\text { N } \\
\dot{0} \\
\text { m } \\
-\end{array}$ & 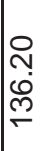 & 令 & ָ̃ & $\mid \begin{array}{c}\hat{N} \\
\stackrel{\mathrm{N}}{\mathrm{N}} \\
-\end{array}$ & $\begin{array}{l}0 \\
N \\
0 \\
0 \\
- \\
-\end{array}$ & $\begin{array}{l}尺 \\
N \\
\dot{e} \\
- \\
-\end{array}$ & 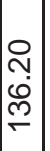 & 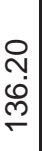 & 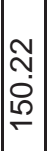 & 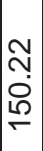 & 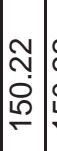 & 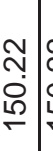 & 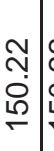 & 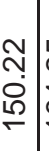 & \begin{tabular}{l}
$\stackrel{N}{N}$ \\
\multirow{+}{0}{} \\
\end{tabular} & & $\mid \begin{array}{c}m \\
m \\
\dot{0} \\
\stackrel{N}{1}\end{array}$ & $\begin{array}{l}0 \\
\text { N } \\
\text { Nิ } \\
\text { N }\end{array}$ \\
\hline 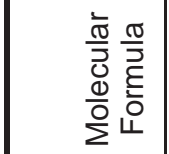 & $\left|\begin{array}{c}0 \\
0 \\
0 \\
1 \\
0 \\
0\end{array}\right|$ & $\begin{array}{l}0 \\
0 \\
0_{1} \\
0 \\
0\end{array}$ & $\left|\begin{array}{c}0 \\
\stackrel{N}{N} \\
\frac{1}{O} \\
0\end{array}\right|$ & \begin{tabular}{l}
0 \\
\multirow{N}{N}{} \\
I \\
0
\end{tabular} & 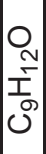 & $\left|\begin{array}{c}0 \\
\stackrel{N}{T} \\
\\
0 \\
0\end{array}\right|$ & $\mid \begin{array}{l}0 \\
\text { N } \\
\frac{N}{1} \\
0 \\
0\end{array}$ & 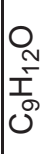 & $\mid \begin{array}{l}0 \\
0 \\
⿱ 亠 䒑 \\
1 \\
0 \\
0\end{array}$ & $\mid \begin{array}{l}0 \\
0 \\
1 \\
1 \\
0 \\
0\end{array}$ & $\left|\begin{array}{c}0 \\
0 \\
1 \\
1 \\
0 \\
0\end{array}\right|$ & 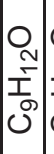 & $\begin{array}{l}0 \\
\stackrel{N}{N} \\
\frac{1}{\sigma} \\
0\end{array}$ & \begin{tabular}{|}
0 \\
$\stackrel{N}{N}$ \\
$\frac{1}{9}$ \\
0 \\
0
\end{tabular} & \begin{tabular}{l}
0 \\
\multirow{N}{T}{} \\
\\
0
\end{tabular} & $\mid \begin{array}{c}0 \\
+ \\
\vdots \\
\vdots \\
0 \\
0 \\
0\end{array}$ & 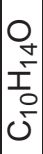 & 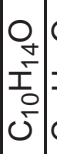 & 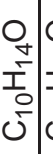 & $\begin{array}{l}0 \\
\vdots \\
\pm \\
0 \\
0\end{array}$ & $\begin{array}{l}0 \\
\vdots \\
\pm \\
\\
\vdots \\
\vdots\end{array}$ & $\begin{array}{l}0 \\
\frac{0}{1} \\
\frac{1}{ \pm} \\
j\end{array}$ & & 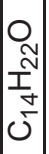 & $\begin{array}{l}0 \\
\text { On } \\
\frac{1}{1} \\
\frac{10}{10} \\
0\end{array}$ \\
\hline 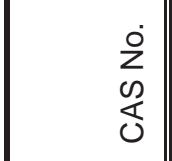 & 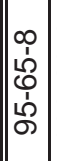 & $\mid \begin{array}{l}0 \\
0 \\
0 \\
0 \\
0 \\
0 \\
0 \\
0\end{array}$ & 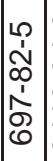 & 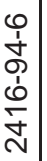 & 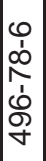 & \begin{tabular}{|c}
0 \\
0 \\
0 \\
$\underline{1}$ \\
1 \\
$\hat{N}$ \\
$\hat{N}$
\end{tabular} & 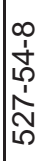 & 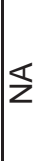 & $\begin{array}{l}0 \\
0 \\
8 \\
0 \\
1 \\
\varnothing \\
\varnothing\end{array}$ & 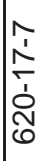 & 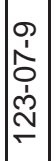 & 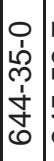 & 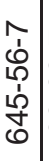 & $\begin{array}{l}1 \\
\dot{1} \\
0 \\
\infty \\
\infty \\
\infty\end{array}$ & $\begin{array}{l}\infty \\
1 \\
\infty \\
\infty \\
\dot{1} \\
\stackrel{2}{ }\end{array}$ & $\begin{array}{l}\infty \\
1 \\
\tilde{N} \\
1 \\
\infty \\
\widetilde{D} \\
\underline{-}\end{array}$ & 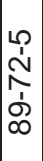 & 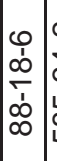 & 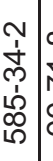 & $\begin{array}{l}\infty \\
\frac{1}{T} \\
\dot{S} \\
\sigma\end{array}$ & 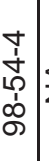 & $\Sigma$ & & 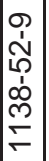 & $\begin{array}{l}0 \\
1 \\
1 \\
0 \\
0 \\
0 \\
\text { N } \\
-1\end{array}$ \\
\hline $\begin{array}{l}\overline{\widetilde{J}} \\
\stackrel{\stackrel{E}{E}}{\Phi} \\
\bar{U} \\
\end{array}$ & 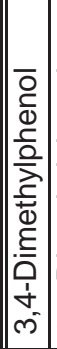 & 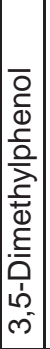 & 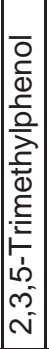 & 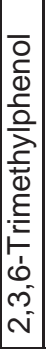 & 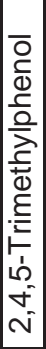 & 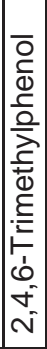 & 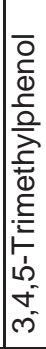 & 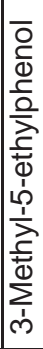 & 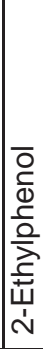 & 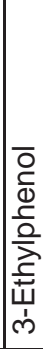 & 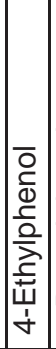 & 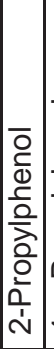 & 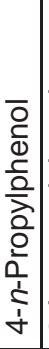 & 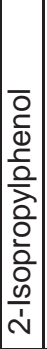 & 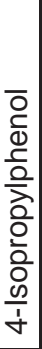 & 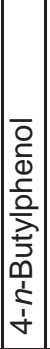 & 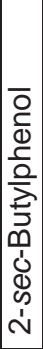 & 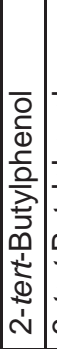 & 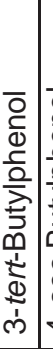 & 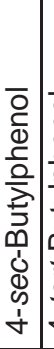 & 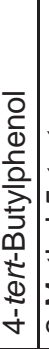 & 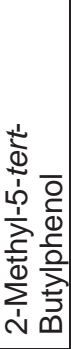 & 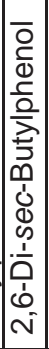 & 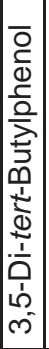 & 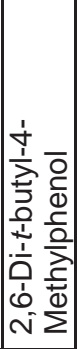 \\
\hline i & $\stackrel{N}{N}$ & $\hat{N}$ & $\stackrel{\infty}{N}$ & $\vec{N}$ & |లি & $\bar{m}$ & ల్ল & $m$ & m) & 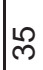 & @ & $\mid \hat{m}$ & 㐫 & m & 乎 & $\forall$ & $\forall$ & क् & 寸 $S$ & ᄂ) & $\varphi$ & f & F & 尔 & i̊ \\
\hline
\end{tabular}




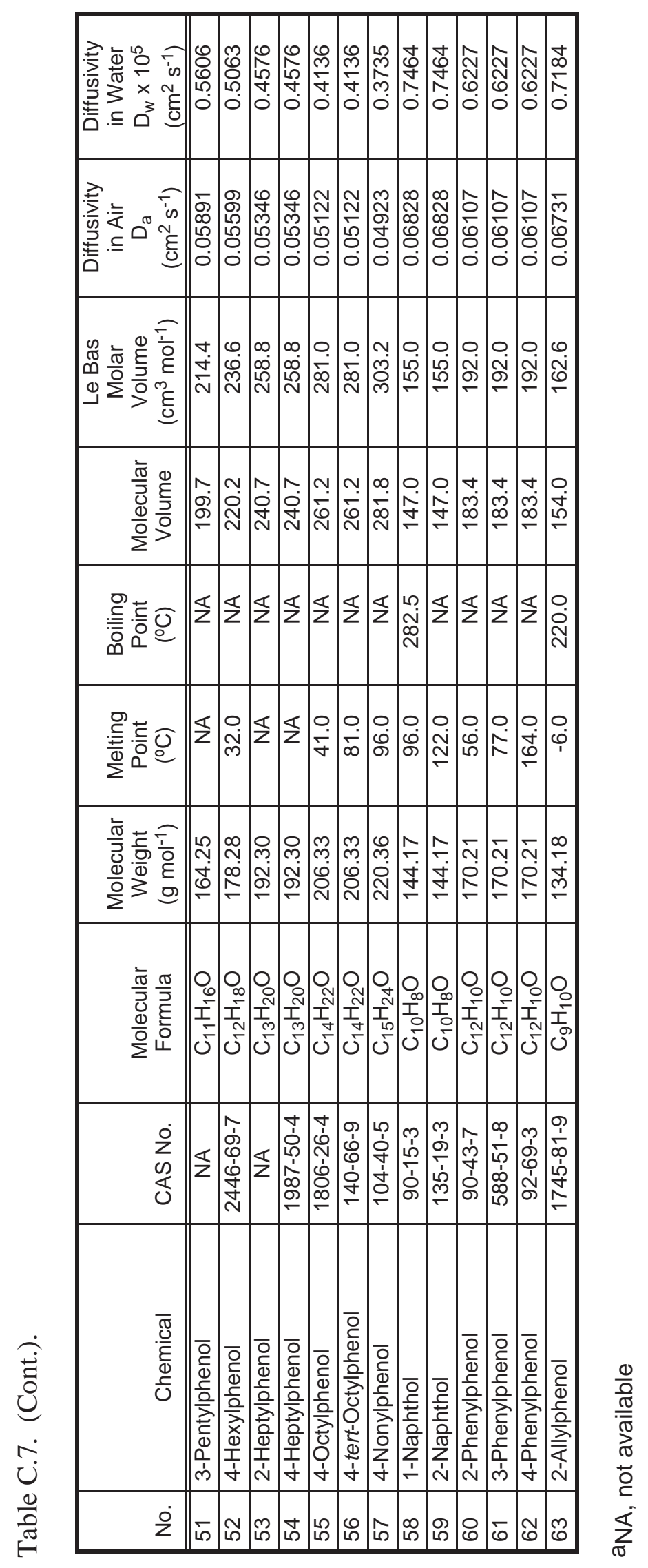




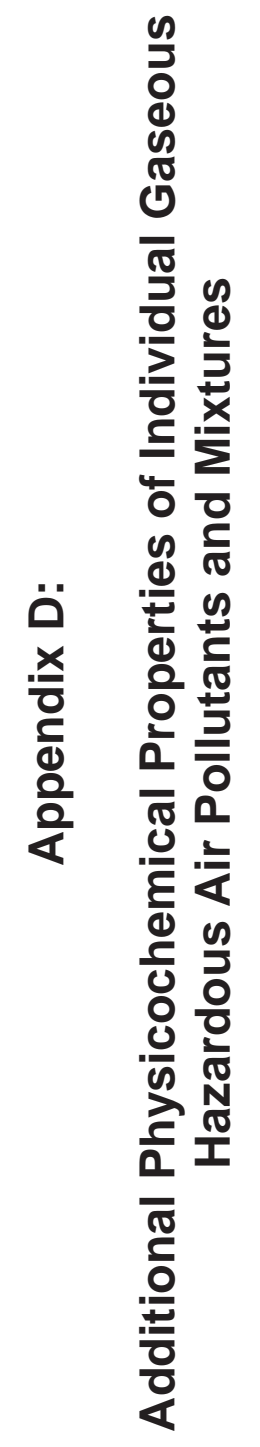




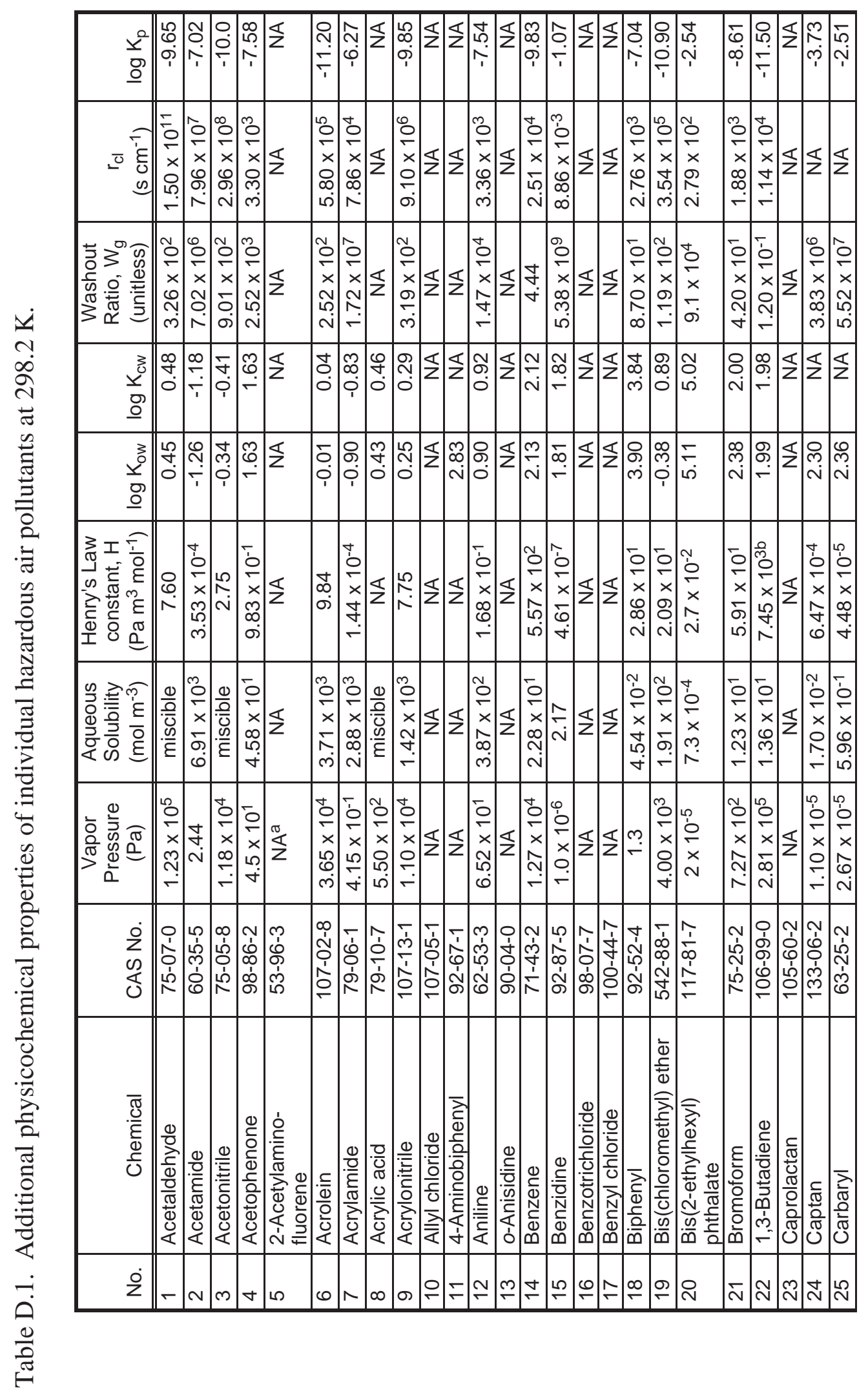




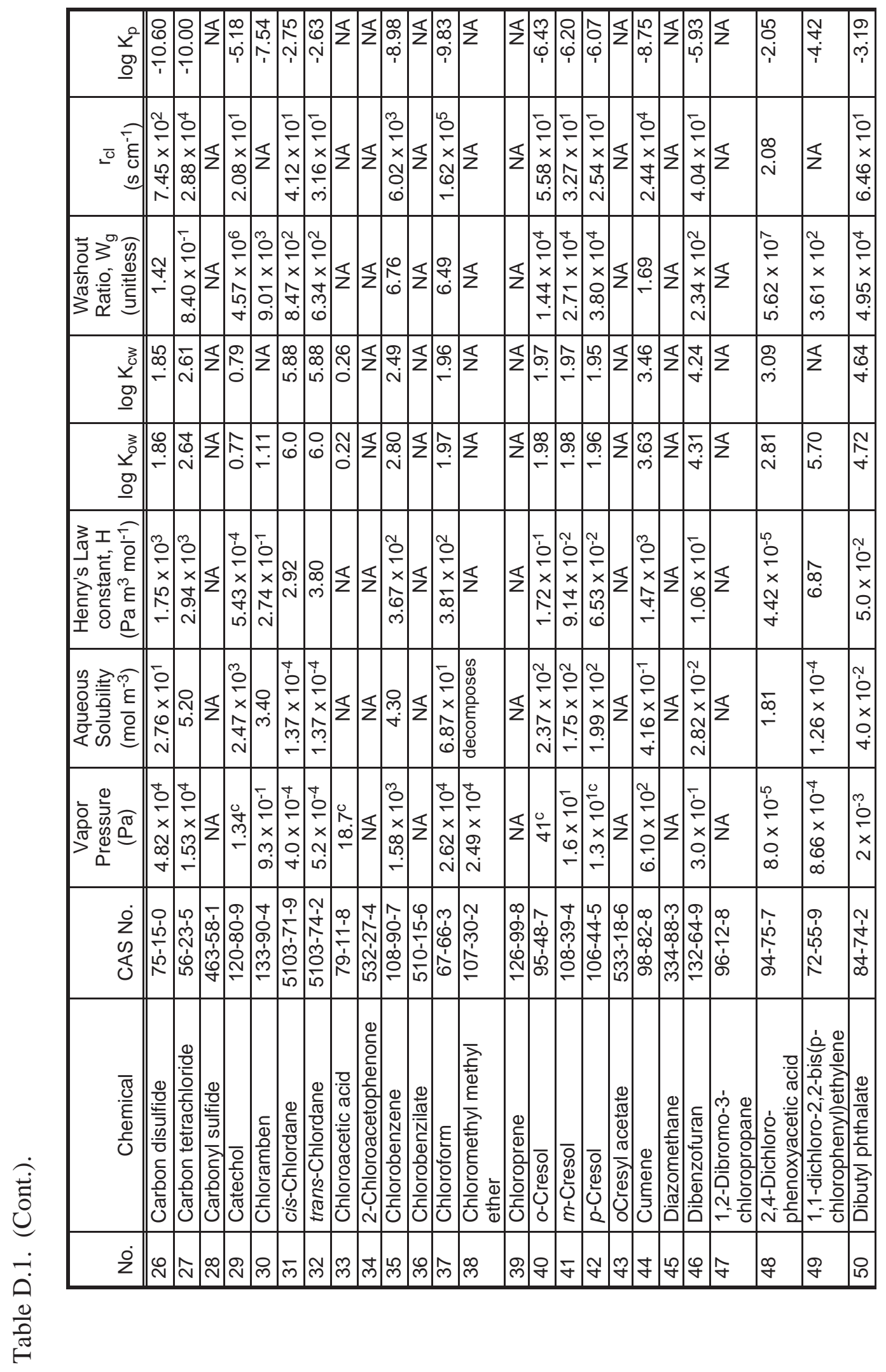




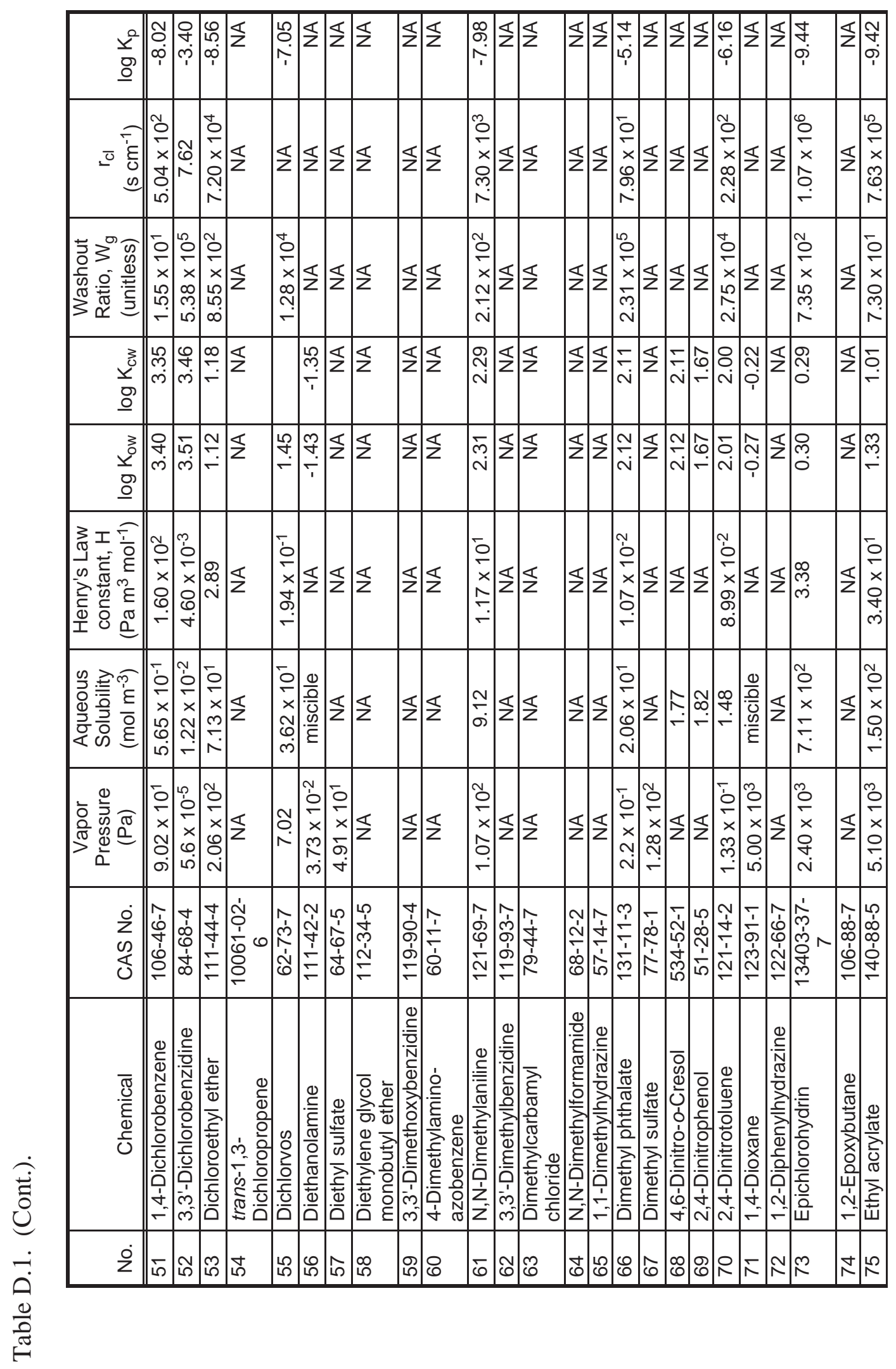




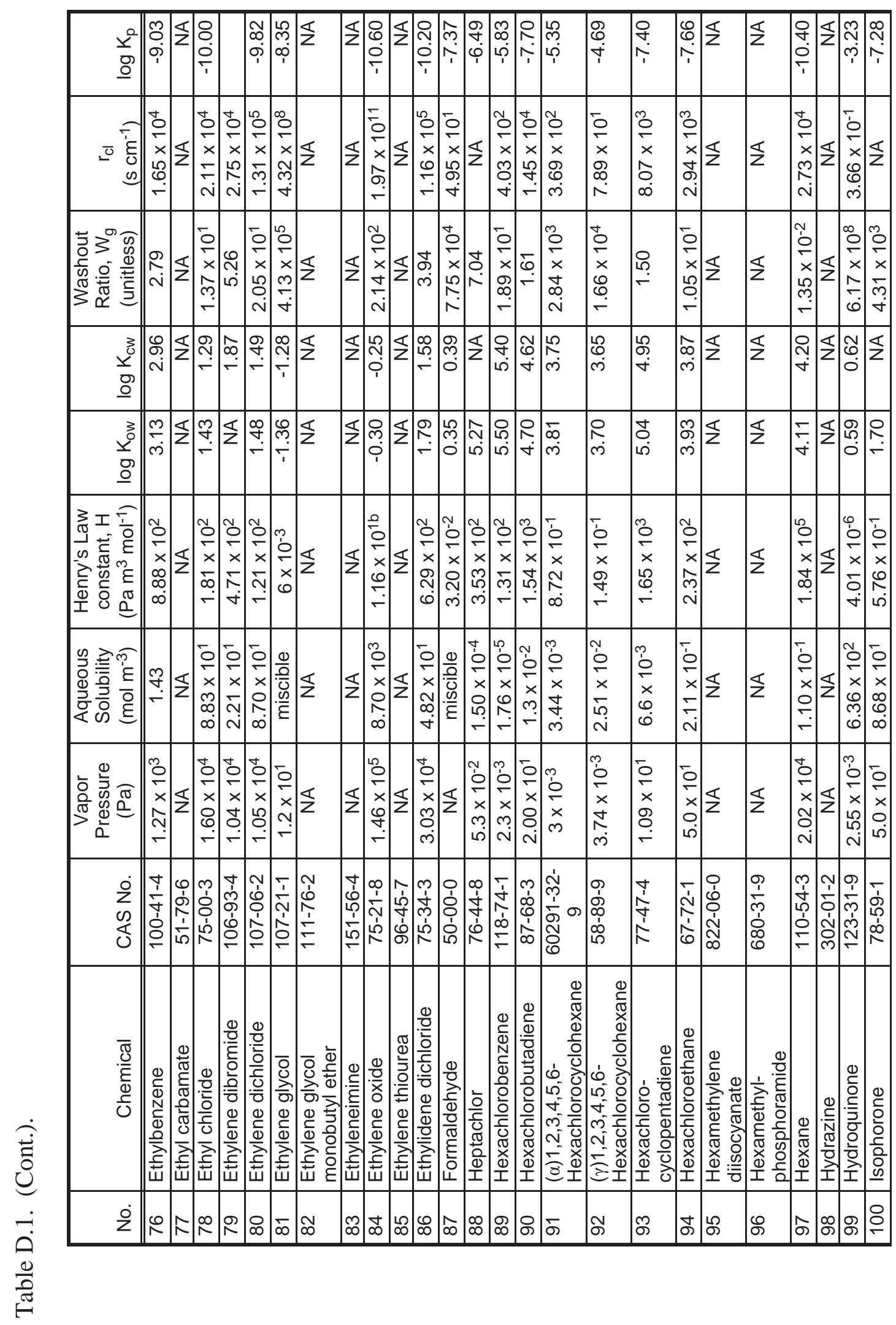




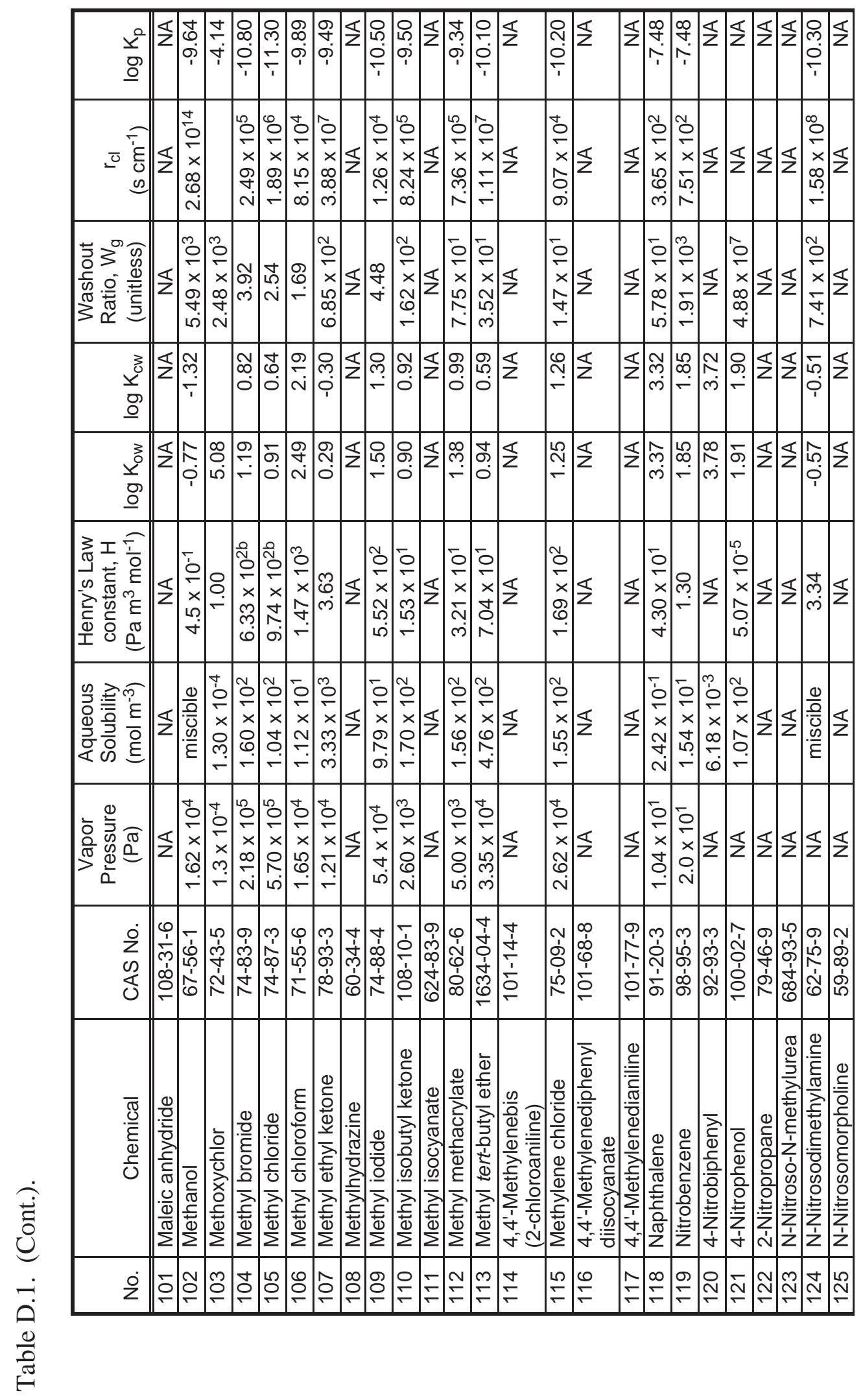




\begin{tabular}{|c|c|c|c|c|c|c|c|c|c|c|c|c|c|c|c|c|c|c|c|c|c|}
\hline $\begin{array}{l}x^{2} \\
\text { 으 }\end{array}$ & {$\left[\begin{array}{l}0 \\
0 \\
p \\
p \\
p \\
1\end{array}\right]$} & & $\begin{array}{l}\mathscr{Q} \\
\dot{0} \\
\dot{p}\end{array}$ & \begin{tabular}{|l}
$\mid g$ \\
+ \\
$\varphi$ \\
\end{tabular} & & $\frac{s}{z}$ & & z $\mathbb{z}$ & $\left|\begin{array}{l}0 \\
0 \\
0 \\
0 \\
1\end{array}\right|$ & $\begin{array}{l}\bar{\sigma} \\
\stackrel{\gamma}{ } \\
\end{array}$ & 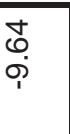 & $\left(\begin{array}{l}0 \\
0 \\
0 \\
\end{array}\right.$ & & & $\mid \begin{array}{l}0 \\
0 \\
0 \\
0 \\
1\end{array}$ & \begin{tabular}{|l}
1 \\
$\infty$ \\
\hdashline \\
$i$ \\
1
\end{tabular} & 离 & $\begin{array}{l}\infty \\
\infty \\
\infty \\
\infty \\
1\end{array}$ & 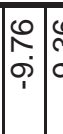 & \begin{tabular}{l|l}
0 & $\mathbb{m}$ \\
$\rho_{1}$ & $\Sigma$
\end{tabular} & 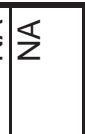 \\
\hline 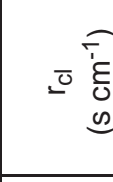 & $\S$ & & 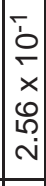 & $\mid$\begin{tabular}{l}
0 \\
\hdashline \\
$\times$ \\
$m$ \\
$m$ \\
$m$ \\
\end{tabular} & & & $\mathbb{z}$ & $\mathbb{z} \leq$ & $\left|\begin{array}{c}0 \\
0 \\
x \\
x \\
10 \\
ن \\
ن\end{array}\right|$ & & $\S$ & $\mid \begin{array}{l}0 \\
0 \\
x \\
\sigma \\
\sigma \\
-\end{array}$ & $\sum \mathbb{c}$ & 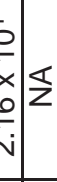 & 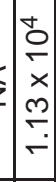 & $\mid \begin{array}{c}0 \\
0 \\
0 \\
x \\
1 \\
b \\
\omega \\
0\end{array}$ & $\$$ & 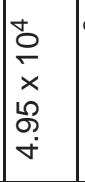 & $\mid \begin{array}{l}n \\
0 \\
0 \\
x \\
x \\
0 \\
0 \\
0 \\
0 \\
0\end{array}$ & 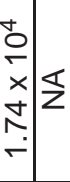 & $\overleftarrow{z}$ \\
\hline 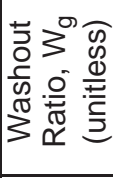 & $\mid \begin{array}{c}10 \\
0 \\
x \\
x \\
0 \\
1 \\
- \\
-\end{array}$ & $\begin{array}{l}y \\
0 \\
x \\
10 \\
0 \\
6 \\
10\end{array}$ & $\mid \begin{array}{l}0 \\
0 \\
x \\
x \\
0 \\
\dot{0} \\
\dot{m}\end{array}$ & 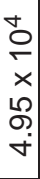 & & & $\mathbb{z}$ & 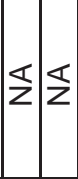 & $\left|\begin{array}{l}0 \\
\bar{x} \\
\dot{m} \\
\bar{e} \\
\dot{m}\end{array}\right|$ & 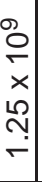 & 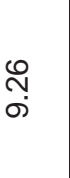 & $\left|\begin{array}{c}\tilde{0} \\
\hdashline \\
x \\
\hat{\infty} \\
\sim \\
\sim\end{array}\right|$ & $\sum \mid$ & 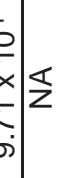 & $=\frac{m}{\infty}$ & $\mid \begin{array}{l}m \\
0 \\
x \\
\dot{j} \\
\dot{f} \\
-\end{array}$ & 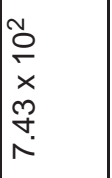 & 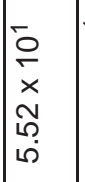 & 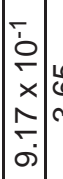 & & $\varangle$ \\
\hline $\begin{array}{l}y_{0}^{3} \\
\text { 음 }\end{array}$ & $\Sigma$ & $\Sigma$ & $\begin{array}{l}\mathscr{Q} \\
\dot{\forall}\end{array}$ & $\stackrel{f}{f}$ & $\mathbb{Z}$ & $\mathbf{z} \mid \S$ & $\mathbb{z}$ & 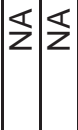 & $\mid$ & $\mathbb{z}$ & $\mathbb{z}$ & $\left|\begin{array}{c}\overline{6} \\
0 \\
\dot{1}\end{array}\right|$ & 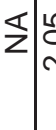 & 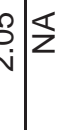 & $\mid \begin{array}{l}\dot{0} \\
\dot{N}\end{array}$ & 产 & $\$$ & . & 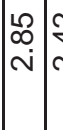 & \begin{tabular}{c|c}
$\mathfrak{v}$ & $\mathbb{Z}$ \\
& $\mathbb{Z}$
\end{tabular} & 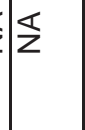 \\
\hline $\begin{array}{l}3 \\
\text { zo } \\
\text { 으 }\end{array}$ & $\mid \begin{array}{l}0 \\
\infty \\
\infty \\
\infty\end{array}$ & $\begin{array}{l}\dot{\sigma} \\
\dot{\sigma} \\
\dot{\sigma}\end{array}$ & $\left|\begin{array}{l}\mid \\
0 \\
0 \\
10\end{array}\right|$ & $\mid \begin{array}{l}0 \\
\stackrel{+}{-} \\
-\end{array}$ & $\mathbb{Z}$ & 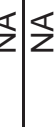 & $\mathbb{z}$ & $|\mathbb{z}| \mathbb{z}$ & $\mid \begin{array}{l}0 \\
0 \\
0 \\
0\end{array}$ & |ำ & ¿̊. & $\mid$ & $\Sigma$ & & $\mid \begin{array}{l}n \\
0 \\
\dot{m}\end{array}$ & ঢִ & 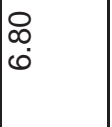 & 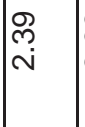 & \begin{tabular}{|l}
$\infty$ \\
$\infty$ \\
$\infty$ \\
\end{tabular} & 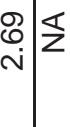 & $\mathbb{z}$ \\
\hline 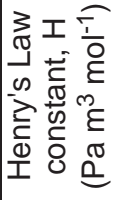 & 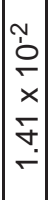 & $\underset{\dot{q}}{\stackrel{q}{+}}$ & $\mid \begin{array}{l}\tilde{y} \\
0 \\
x \\
x \\
\infty \\
\infty \\
\\
\end{array}$ & 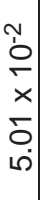 & & $\begin{array}{l}\mathbf{z} \\
\mathbf{z}\end{array}$ & $\S$ & $\S \ll$ & 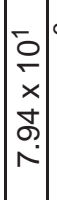 & $\begin{array}{l}0 \\
0 \\
0 \\
x \\
0 \\
0 \\
\vdots \\
-\end{array}$ & 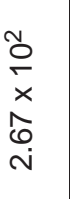 & $\mid$ & 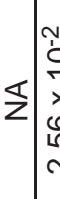 & 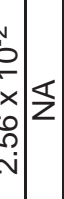 & $=\mid \begin{array}{l}\tilde{y} \\
0 \\
x \\
x \\
0 \\
0 \\
\dot{m}\end{array}$ & \begin{tabular}{c}
$\stackrel{N}{N}$ \\
\hdashline
\end{tabular} & 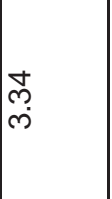 & 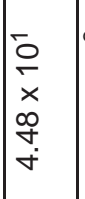 & 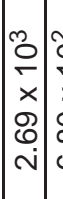 & \begin{tabular}{l|l}
$\tilde{y}$ & \\
0 & \\
$x$ & $\nwarrow$ \\
0 & $\Sigma$ \\
0 \\
0
\end{tabular} & $\Sigma$ \\
\hline 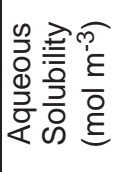 & $\begin{array}{l}\hat{N} \\
0 \\
\sim \\
x \\
0 \\
0 \\
\dot{v} \\
\dot{v}\end{array}$ & $\begin{array}{l}m \\
\dot{0} \\
x \\
x \\
\stackrel{0}{r}\end{array}$ & 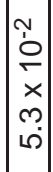 & $\mid \begin{array}{l}v \\
0 \\
x \\
x \\
0 \\
\tilde{m} \\
\sigma\end{array}$ & & $\bar{z} \mid \varangle$ & $\S$ & $\mathbb{z} \mathbb{z}$ & 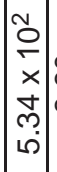 & $\begin{array}{l}8 \\
0 \\
\infty \\
\infty\end{array}$ & \begin{tabular}{l}
$\bar{o}$ \\
$\frac{1}{x}$ \\
$\infty$ \\
$o$ \\
\multirow{i}{*}{}
\end{tabular} & $\left|\begin{array}{c}0 \\
0 \\
0 \\
\times \\
0 \\
\infty \\
\infty\end{array}\right|$ & 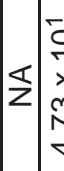 & 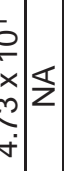 & $=\left[\begin{array}{l}\infty \\
\infty \\
i \\
N\end{array}\right.$ & $\mid \begin{array}{l}- \\
0 \\
\times \\
\infty \\
\infty \\
\\
\end{array}$ & $\begin{array}{l}10 \\
0 \\
\frac{0}{x} \\
0 \\
0\end{array}$ & $\mid \begin{array}{l}0 \\
\frac{0}{x} \\
\hat{x} \\
r\end{array}$ & 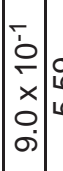 & مْ & $\frac{1}{z}$ \\
\hline 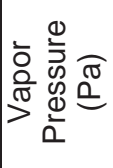 & $\mid$\begin{tabular}{l}
+ \\
$\dot{0}$ \\
\hdashline \\
$\dot{x}$ \\
0 \\
$\dot{0}$ \\
\end{tabular} & $\begin{array}{l}\infty \\
0 \\
0 \\
x \\
0 \\
0 \\
0\end{array}$ & $\mid \begin{array}{l}0 \\
0 \\
0 \\
x \\
6 \\
10 \\
\dot{j}\end{array}$ & $\mid \begin{array}{l}-0 \\
0 \\
x \\
\wedge \\
\dot{\gamma}\end{array}$ & & $\mathbb{z} \mid \mathbb{z}$ & 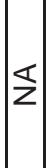 & 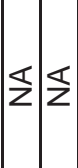 & 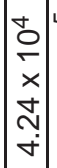 & $\begin{array}{l}\varphi \\
0 \\
0 \\
x \\
0 \\
0 \\
\vdots \\
\vdots\end{array}$ & 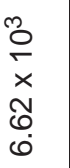 & $\left|\begin{array}{l}0 \\
0 \\
x \\
0 \\
0 \\
\end{array}\right|$ & $\leq$ & $\bar{v}=\mathbb{z}$ & $=$ & $\mid \begin{array}{l}\overline{0} \\
\dot{0} \\
\dot{x} \\
0 \\
\dot{\gamma}\end{array}$ & $\begin{array}{l}\hat{1} \\
\hat{0} \\
x \\
\dot{x} \\
\circ \\
\text { iv }\end{array}$ & 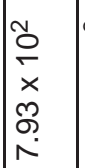 & 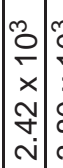 & 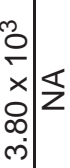 & 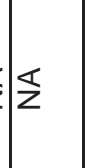 \\
\hline $\begin{array}{l}0 \\
2 \\
0 \\
0\end{array}$ & $\mid \begin{array}{l}N \\
\infty \\
0 \\
0 \\
0 \\
\omega\end{array}$ & $\begin{array}{l}\infty \\
\infty \\
0 \\
0 \\
\grave{1} \\
\infty\end{array}$ & $\begin{array}{l}1 \\
0 \\
0 \\
\infty \\
1 \\
\infty \\
\infty\end{array}$ & 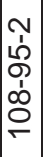 & '. & 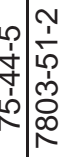 & 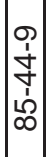 & 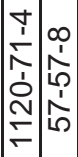 & $\begin{array}{c}0 \\
0 \\
\infty \\
m \\
\tilde{N} \\
\end{array}$ & 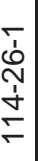 & 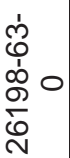 & 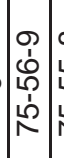 & 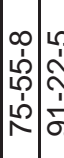 & 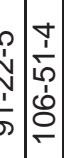 & 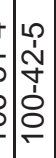 & $\begin{array}{l}\infty \\
\text { ô } \\
0 \\
0 \\
6 \\
\sigma\end{array}$ & $\begin{array}{l}0 \\
1 \\
0 \\
1 \\
0 \\
\dot{1} \\
\\
\end{array}$ & 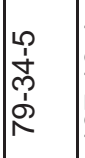 & 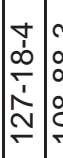 & 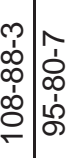 & 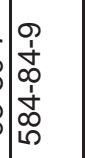 \\
\hline $\begin{array}{l}\overline{\widetilde{J}} \\
\frac{.0}{E} \\
\frac{D}{U} \\
\frac{\mathbb{U}}{U}\end{array}$ & 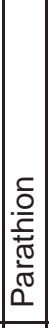 & 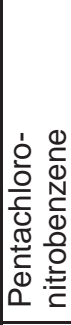 & 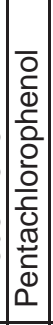 & \begin{tabular}{|l} 
\\
\\
$\overline{0}$ \\
$\frac{\sigma}{0}$ \\
$\frac{\Phi}{\alpha}$ \\
\end{tabular} & 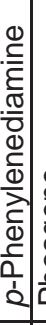 & 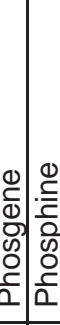 & 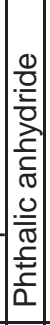 & 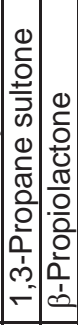 & \begin{tabular}{|l|}
$\frac{0}{0}$ \\
$\frac{2}{2}$ \\
$\frac{0}{0}$ \\
$\frac{0}{0}$ \\
$\frac{0}{0}$ \\
$\frac{0}{2}$ \\
$\frac{0}{2}$ \\
0 \\
\end{tabular} & 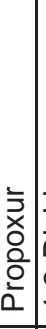 & 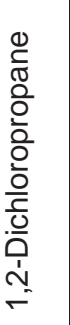 & 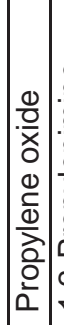 & 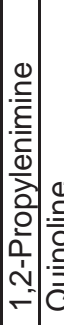 & 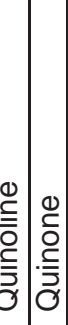 & 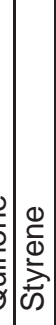 & 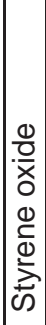 & 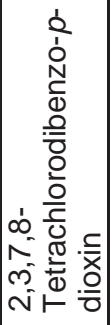 & 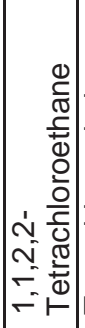 & 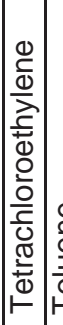 & 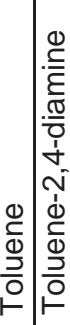 & 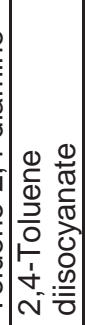 \\
\hline i & 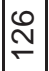 & $\stackrel{N}{N}$ & 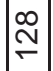 & $\stackrel{\mathscr{T}}{-}$ & প্লি: & 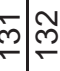 & m & 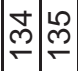 & $\begin{array}{l}0 \\
m \\
-\end{array}$ & $\widehat{\hat{m}}$ & $\stackrel{\infty}{m}$ & প্ল- & 肙 & 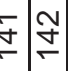 & 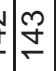 & 专 & $\stackrel{10}{\leftarrow}$ & 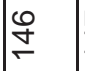 & $\underset{f}{f}$ & 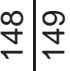 & $\stackrel{\circ}{10}$ \\
\hline
\end{tabular}




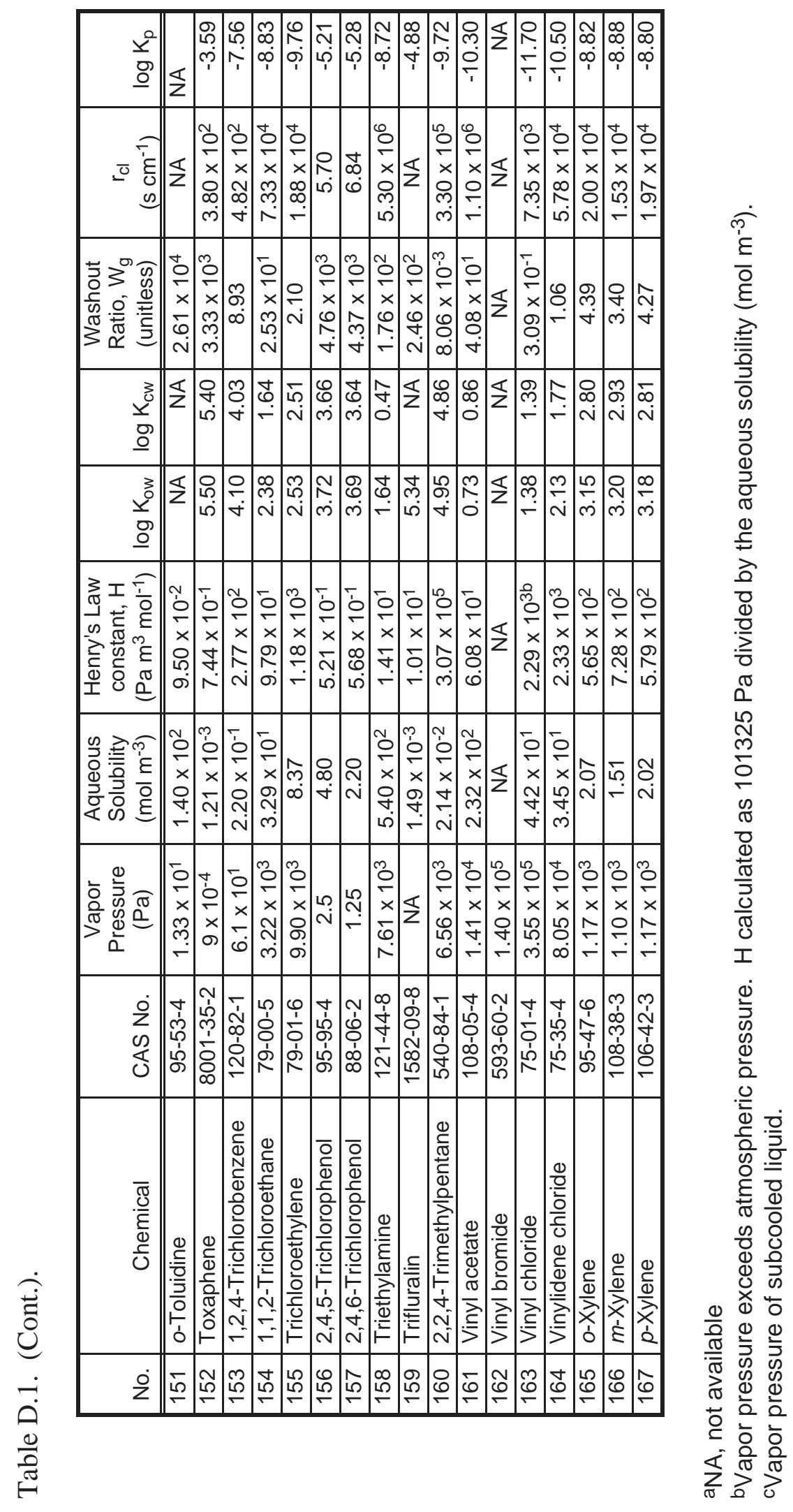




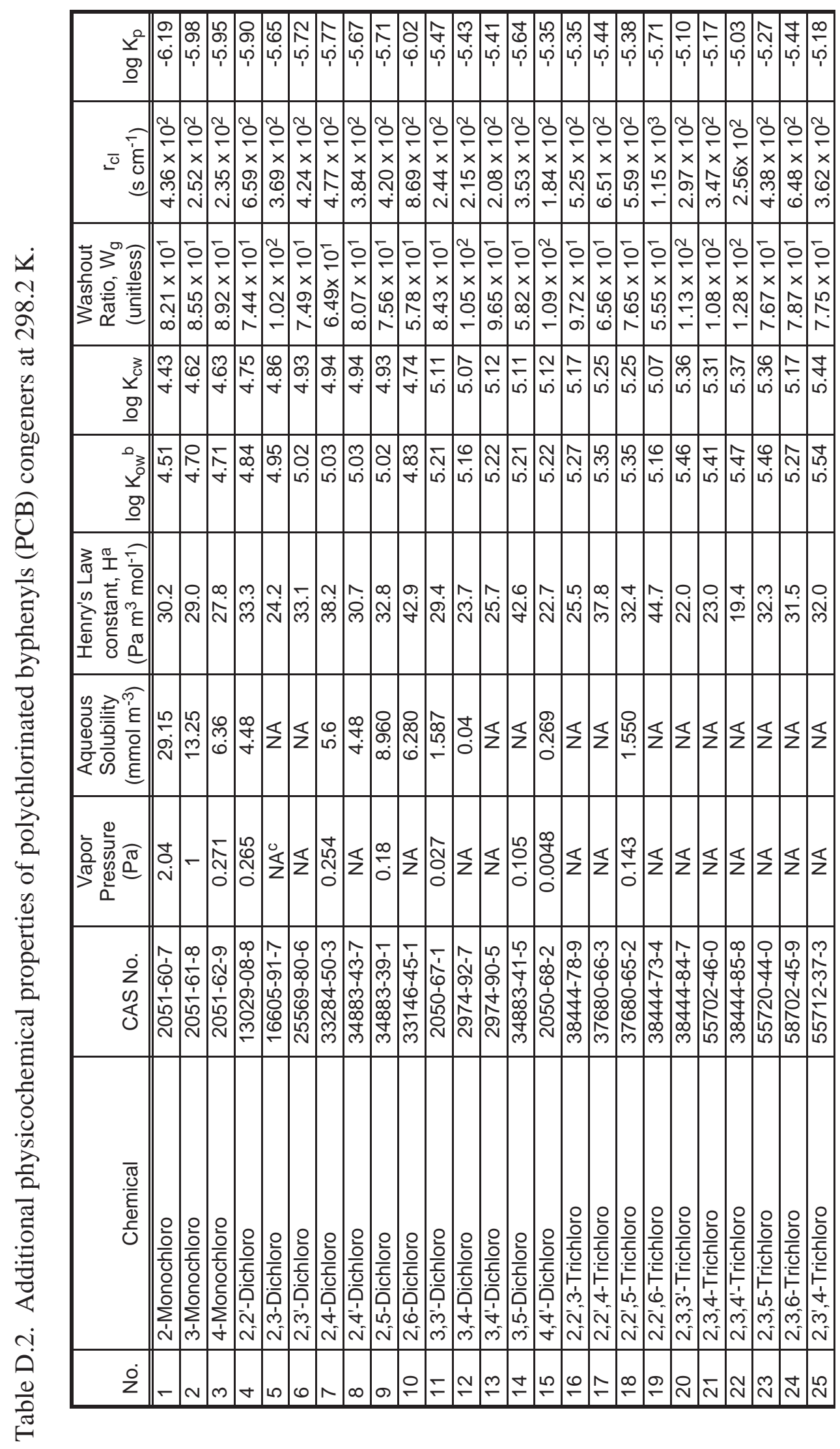




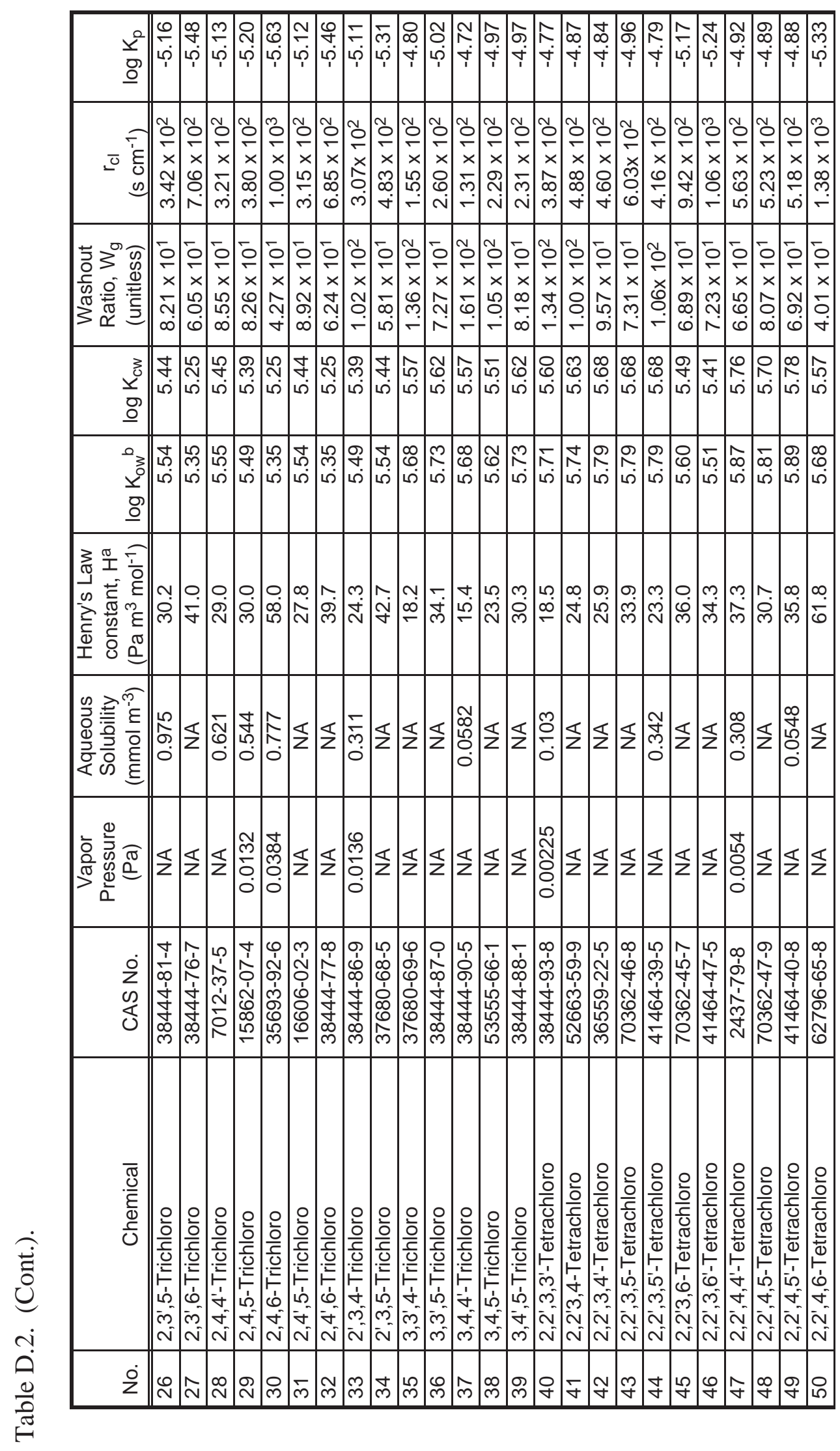




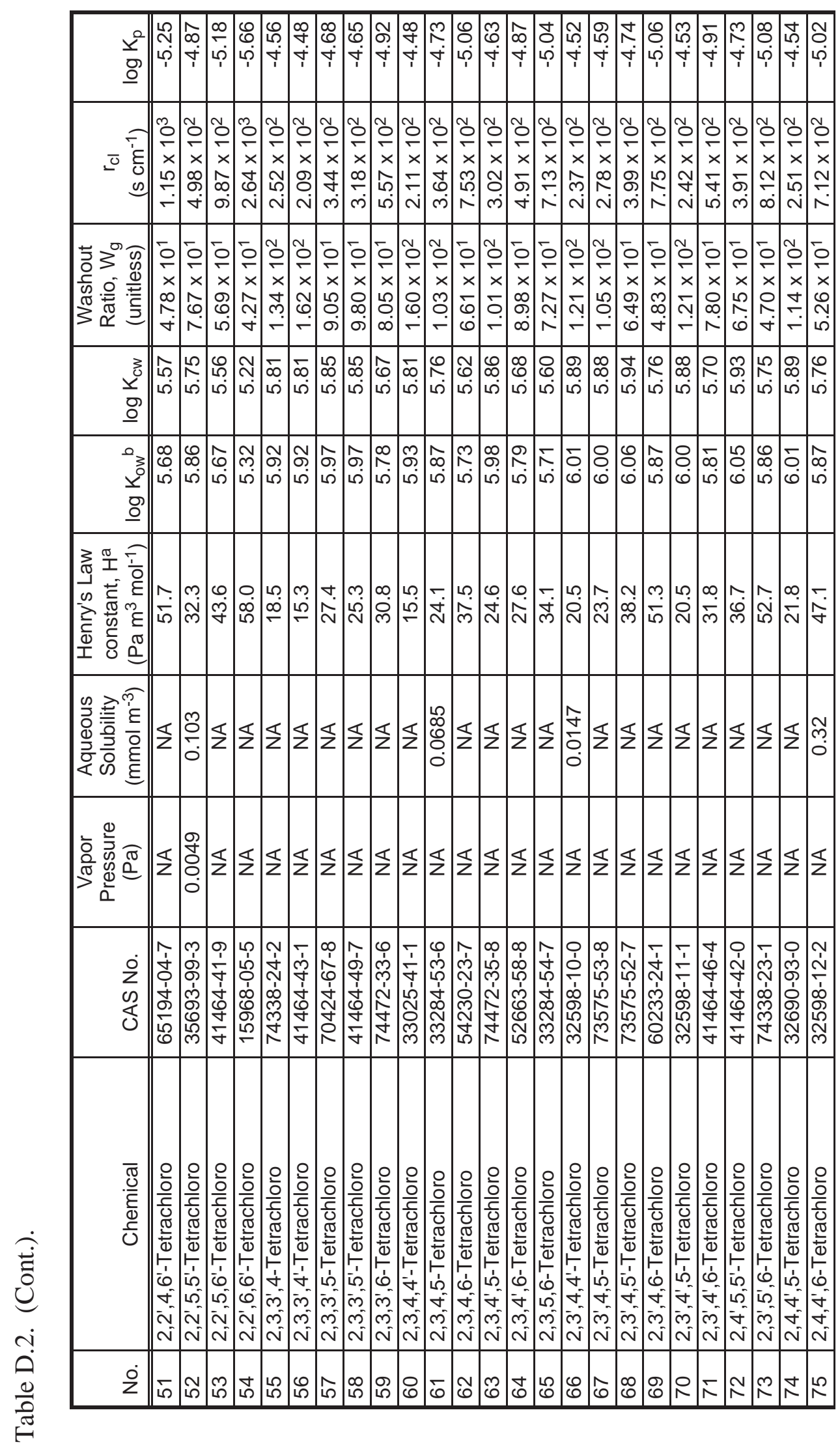




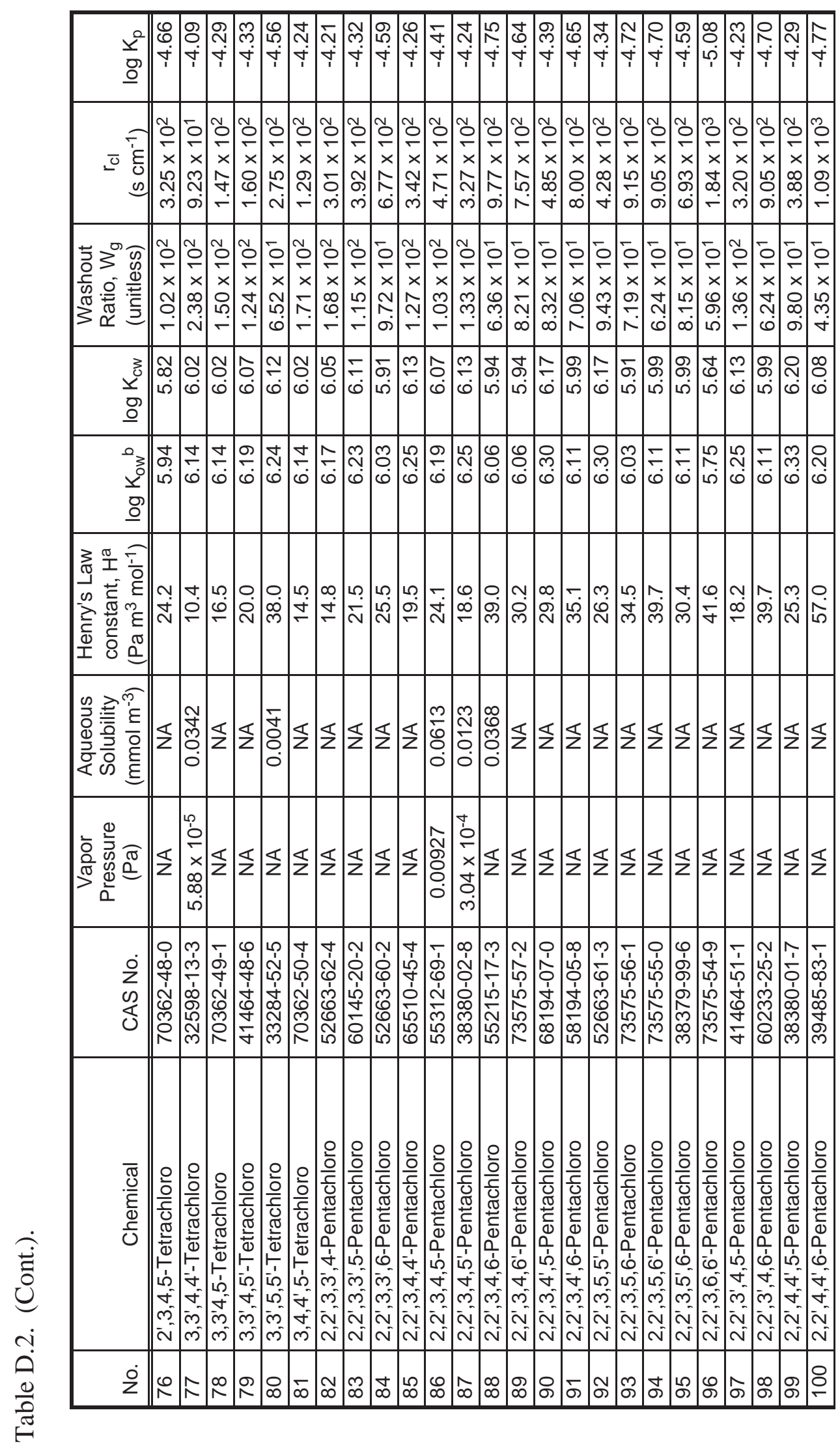




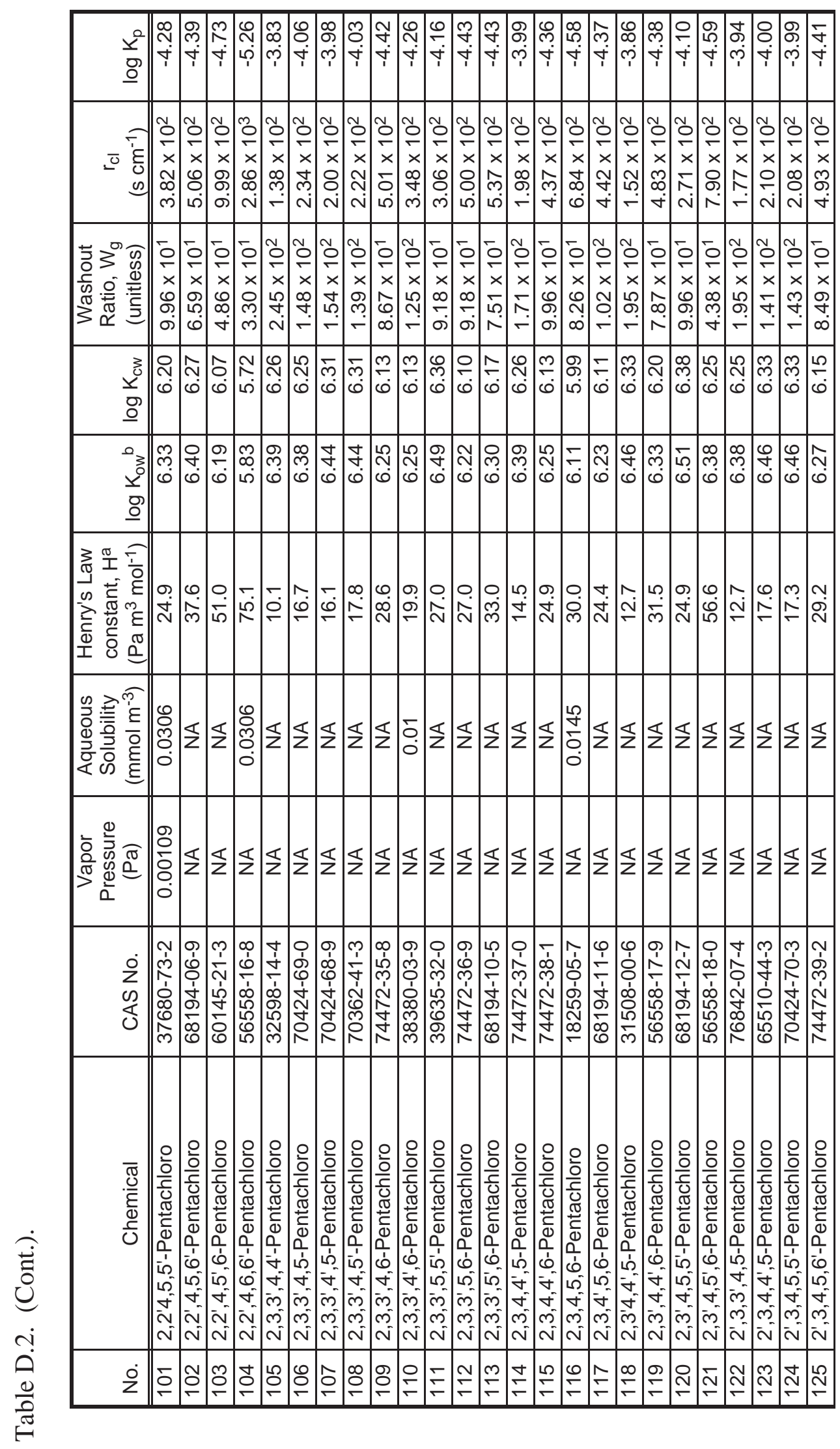




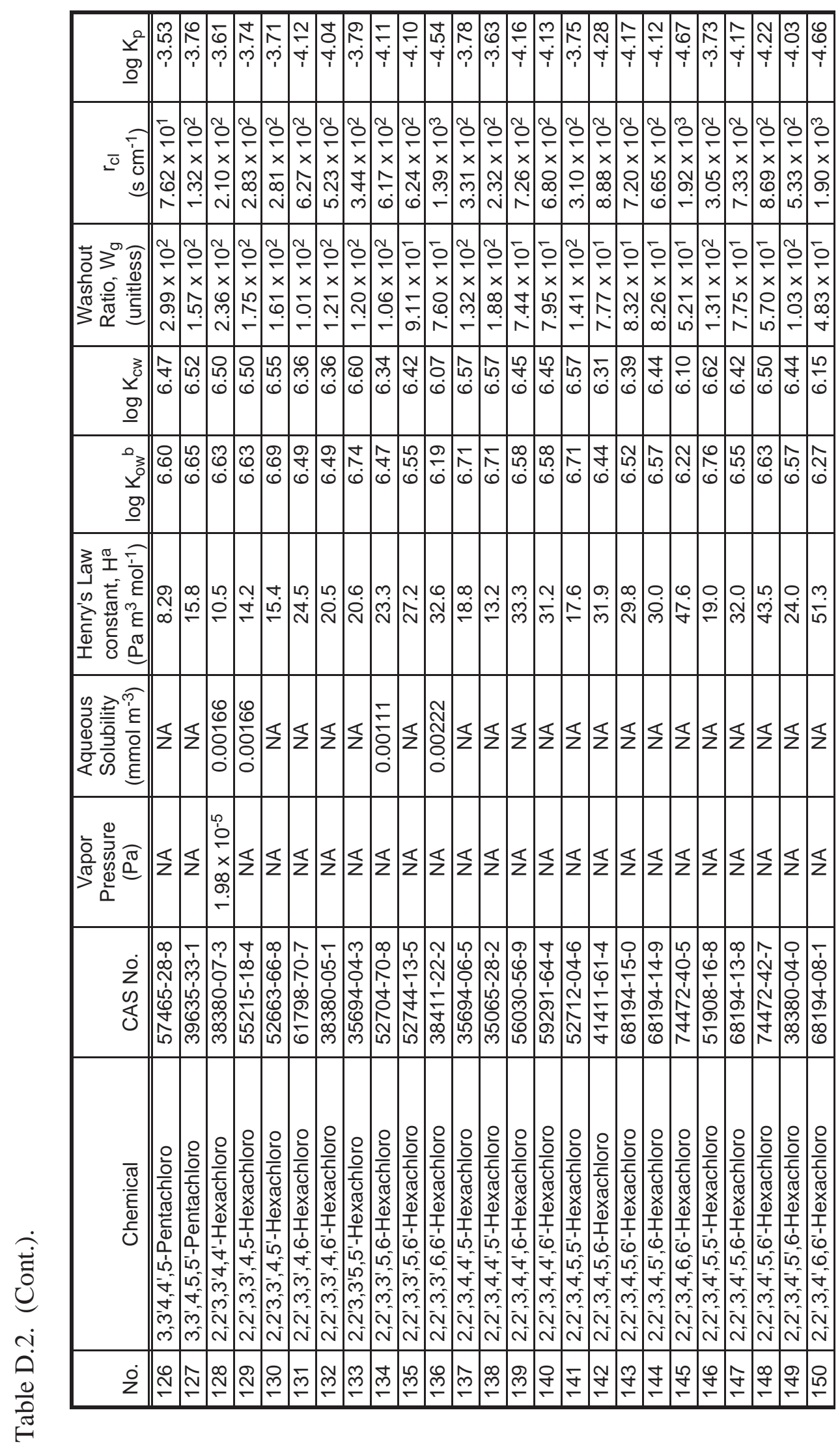




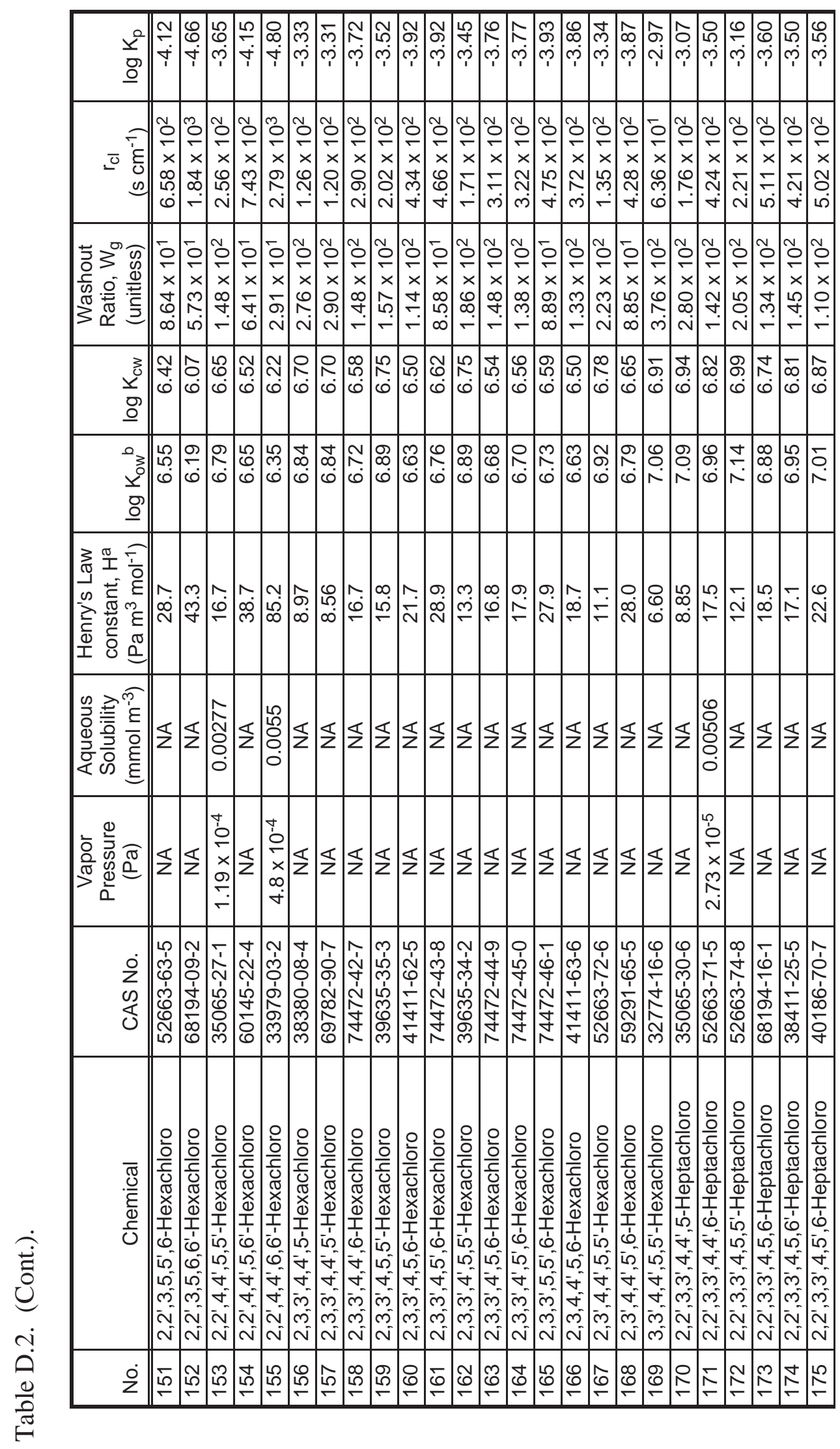




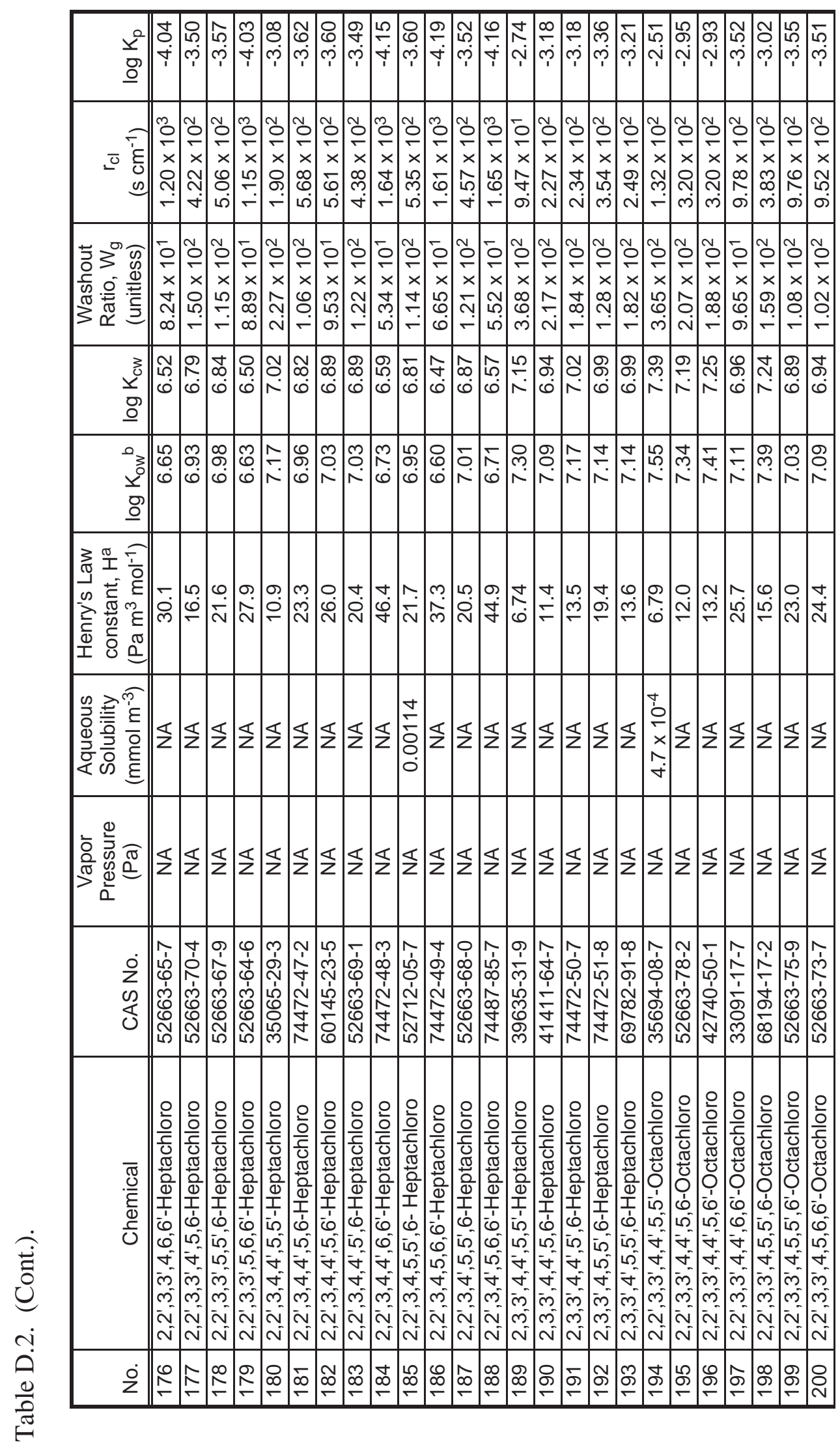




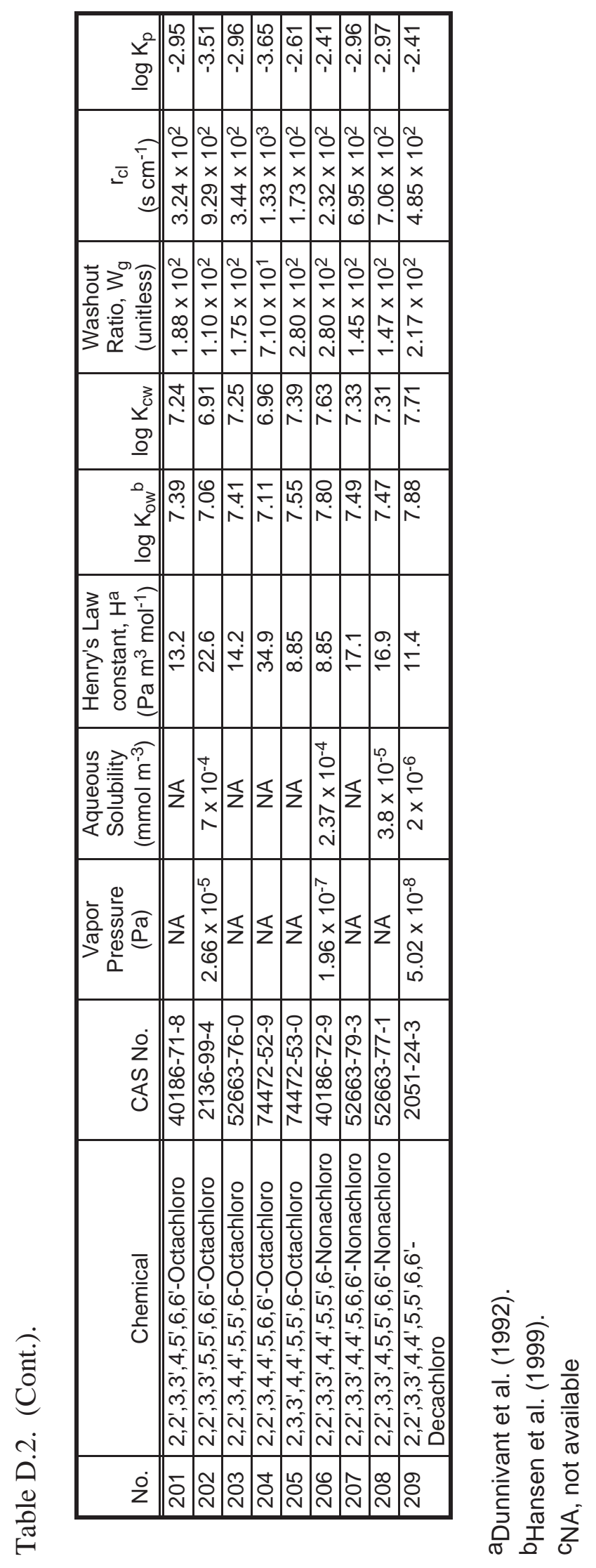

홍 


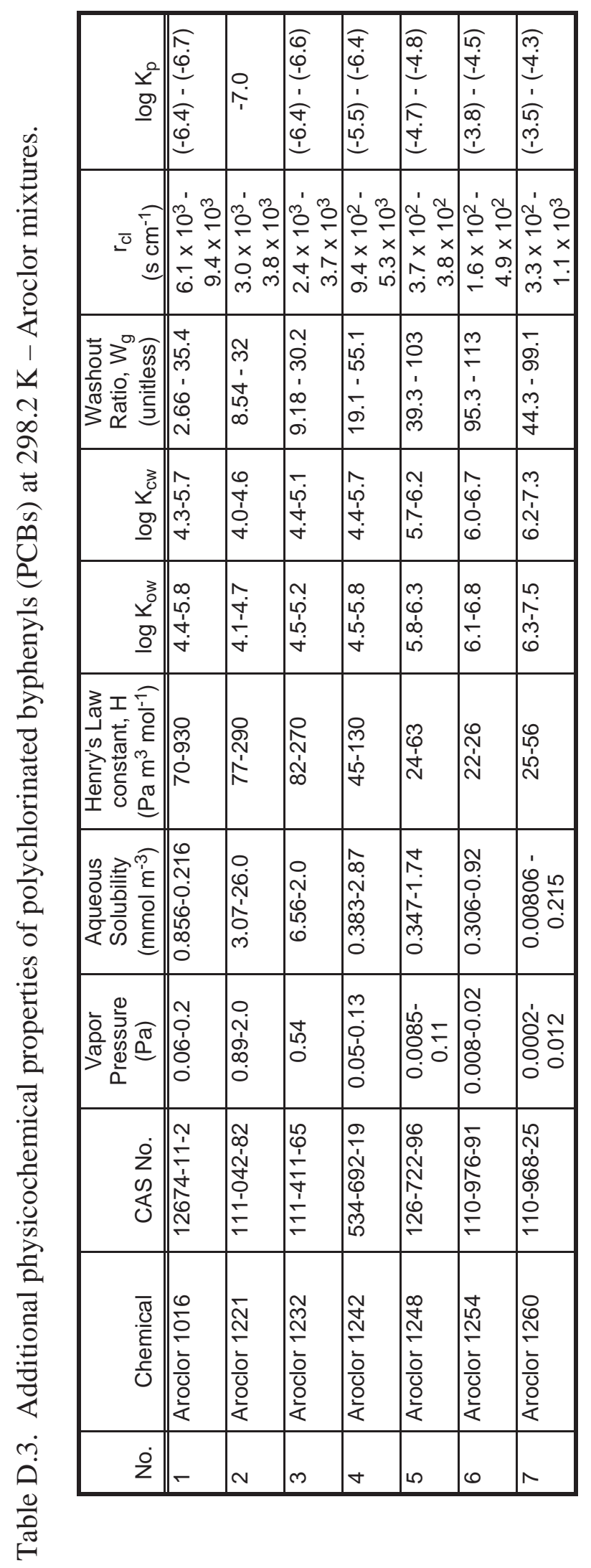




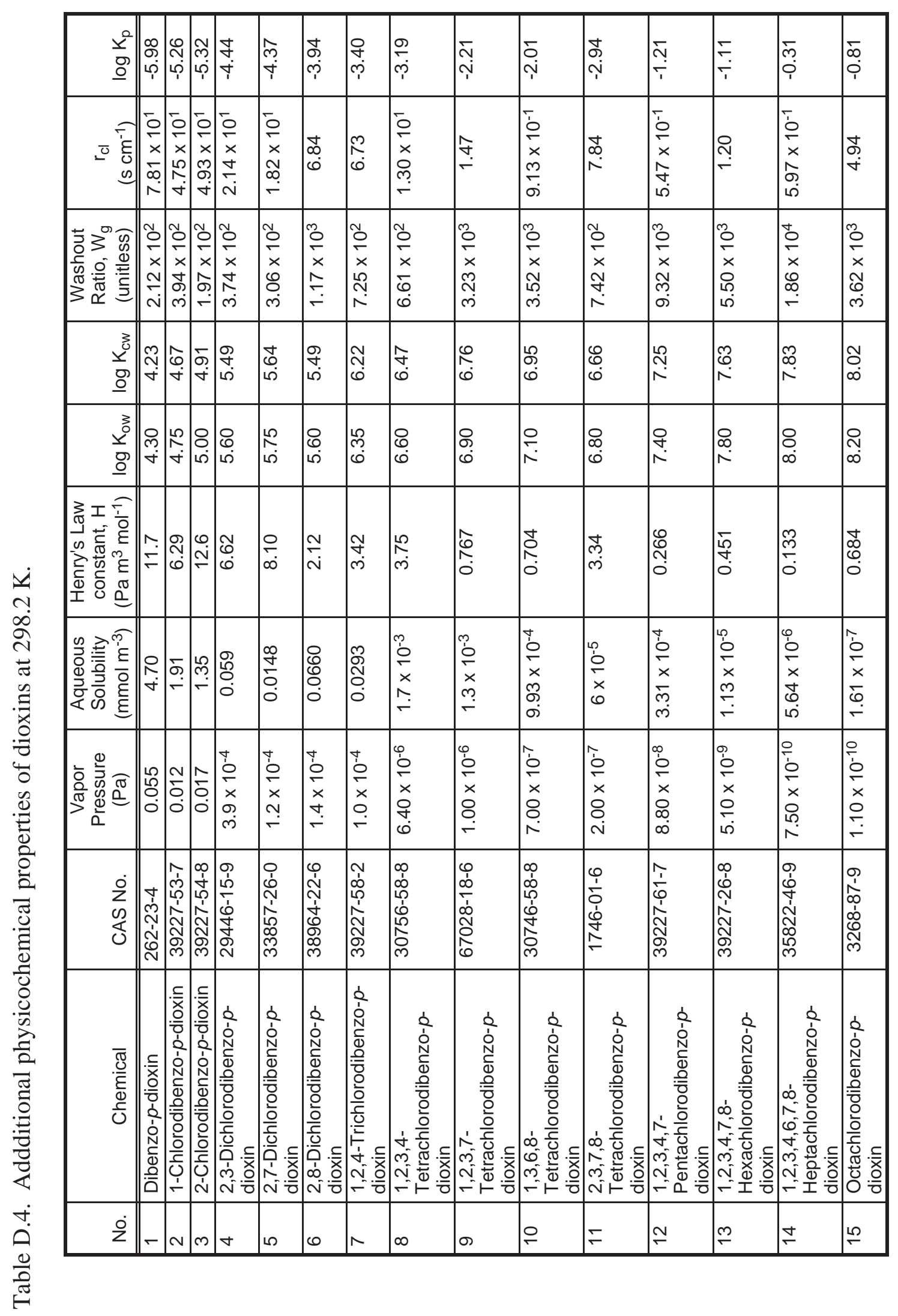




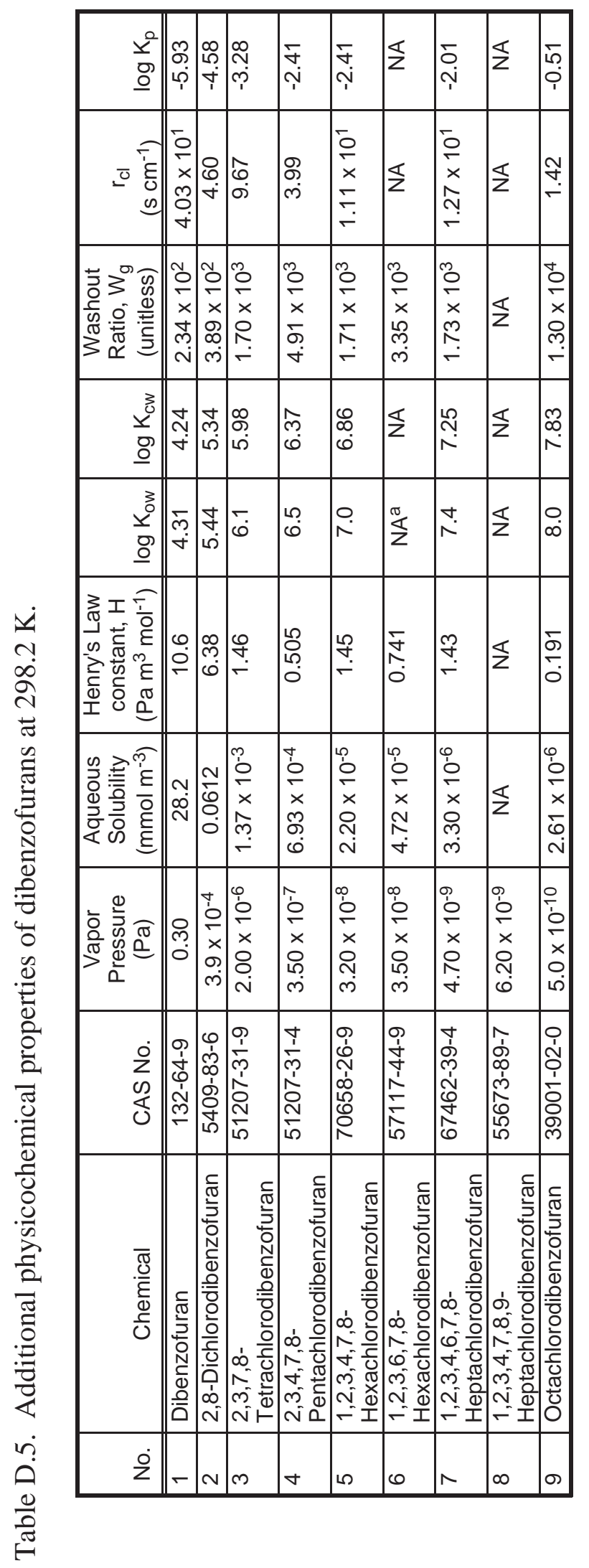




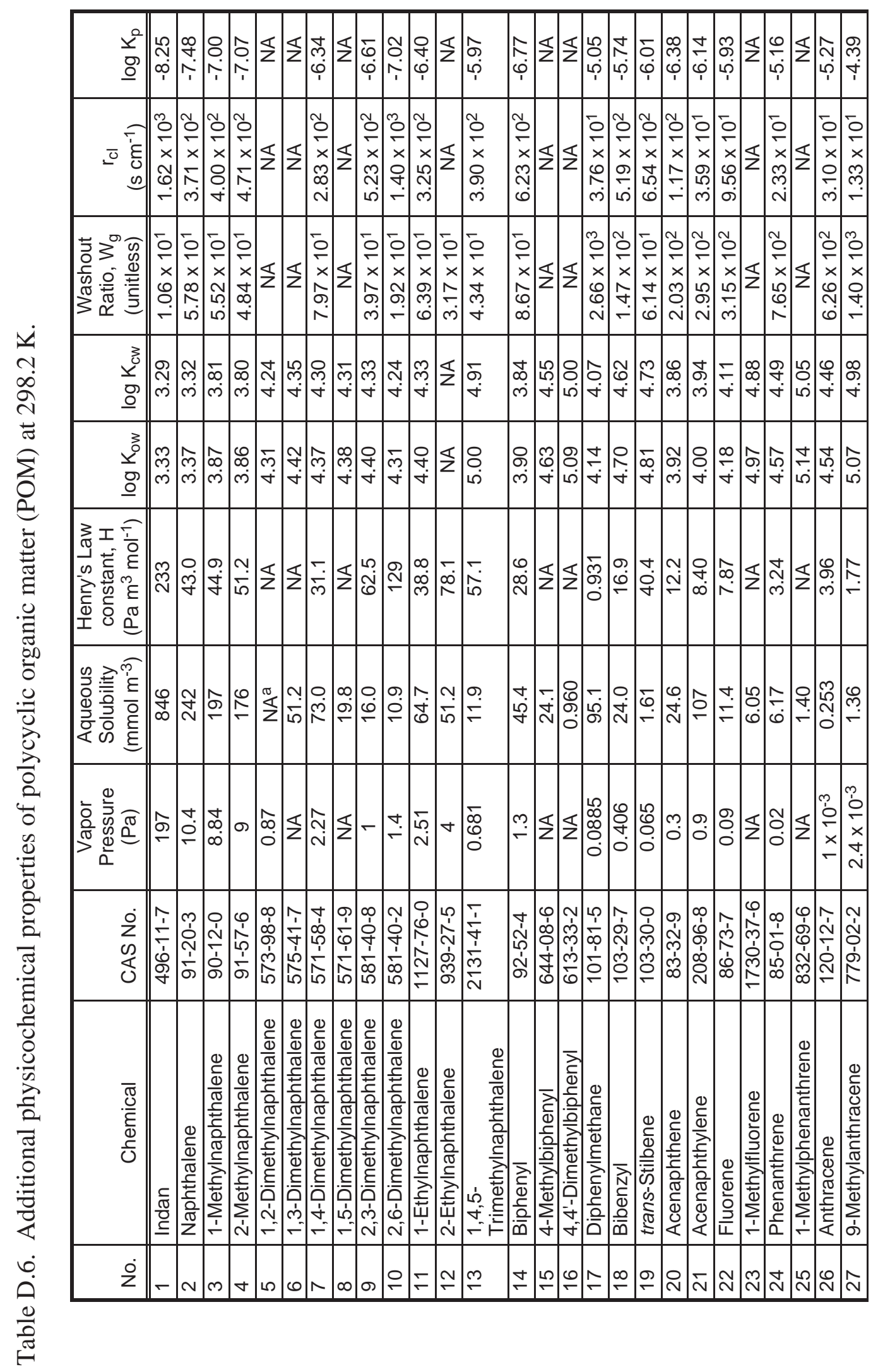




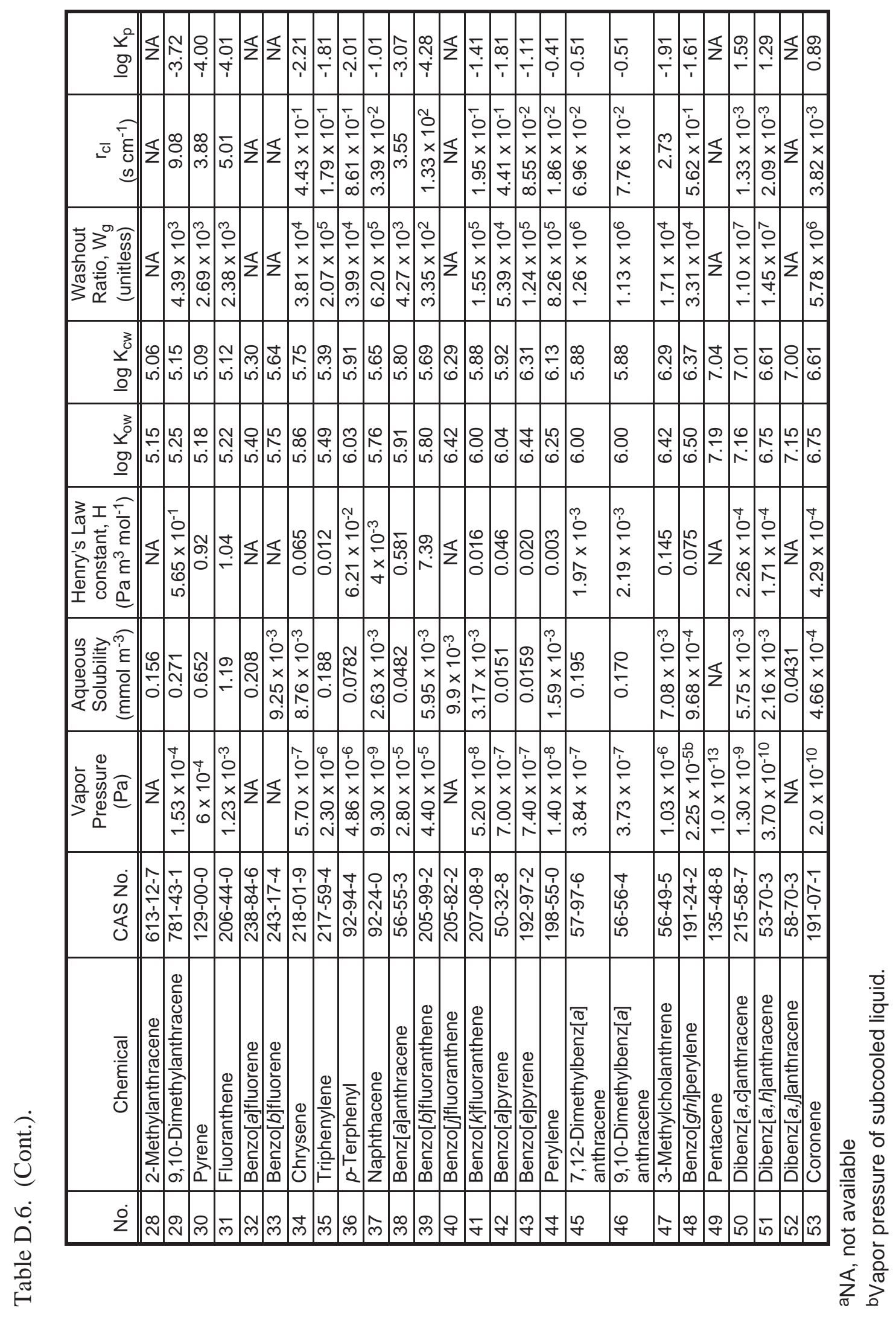




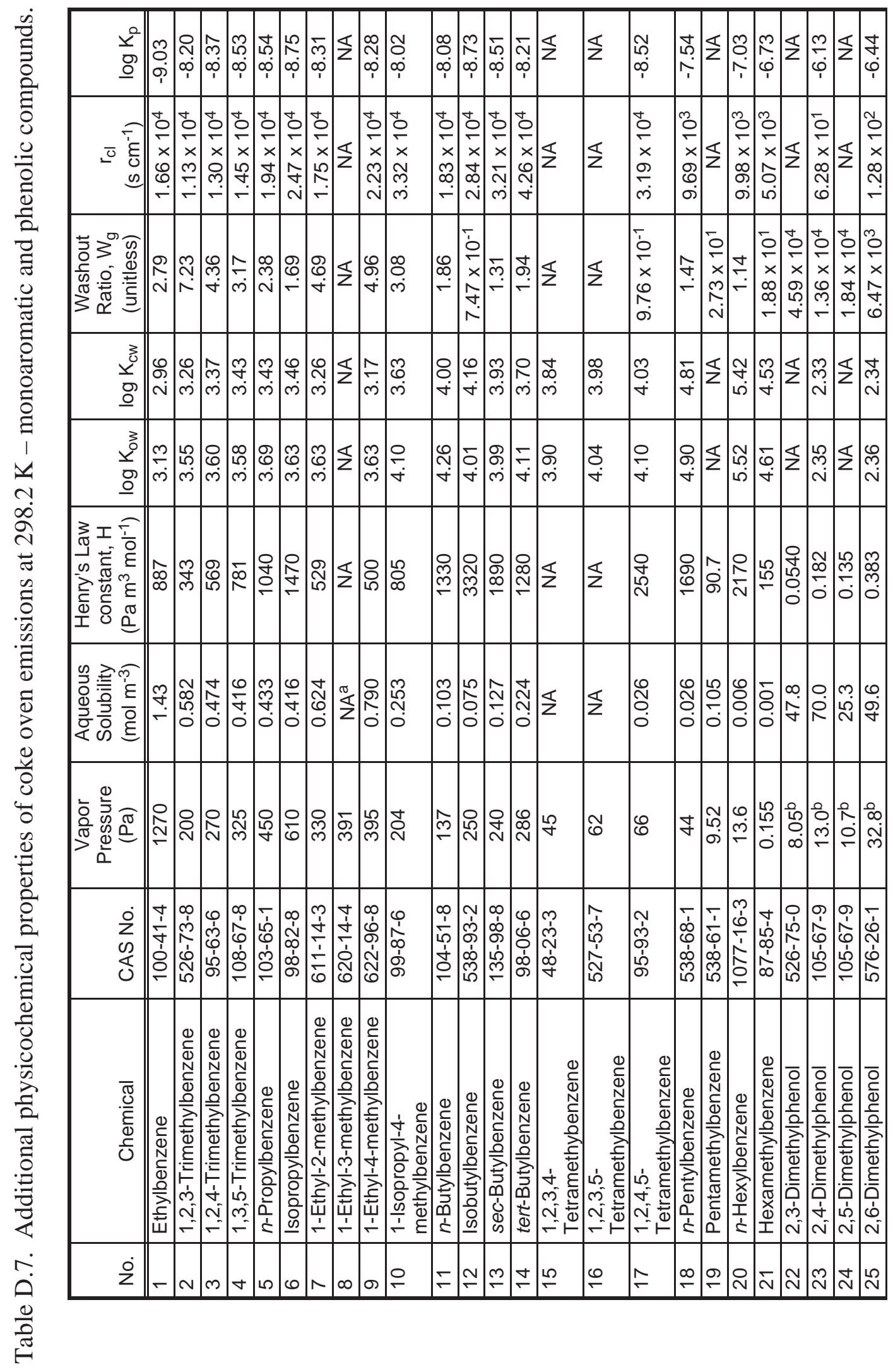




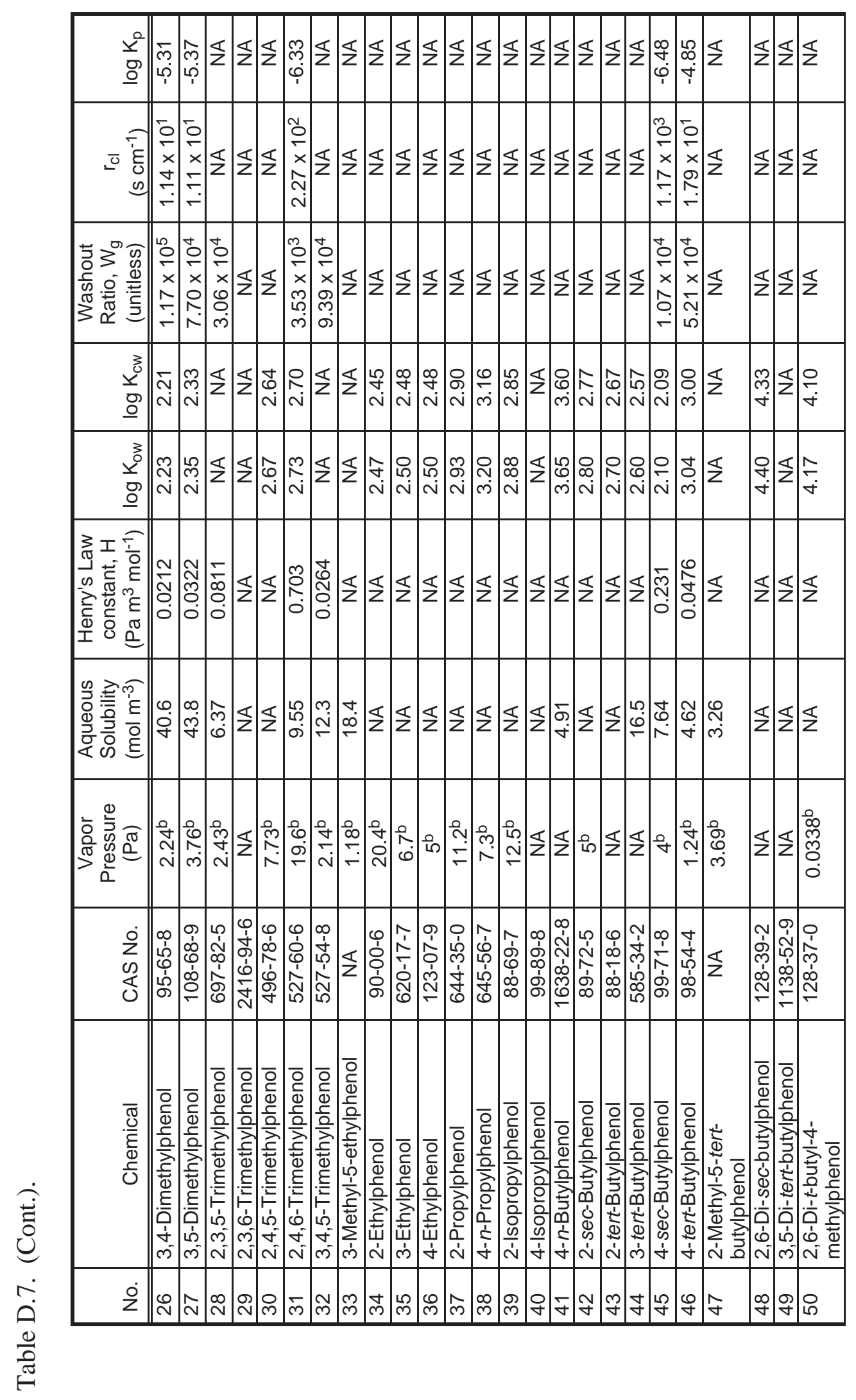




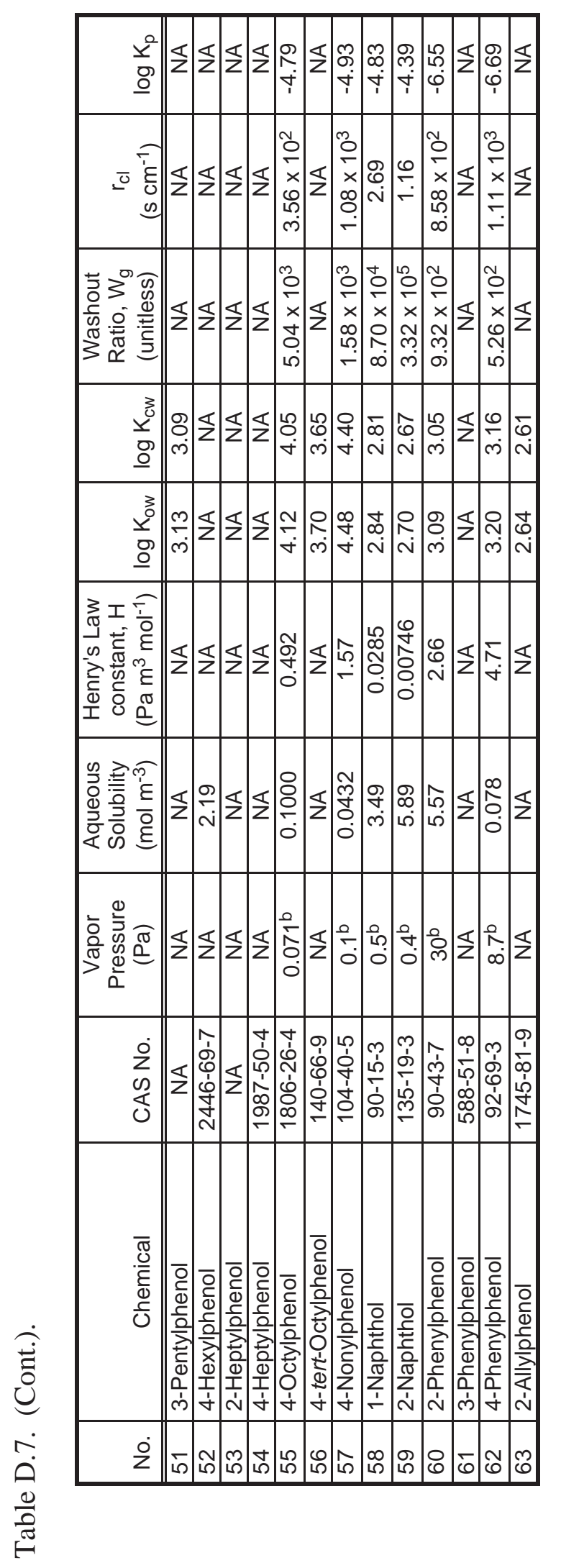

กิ่

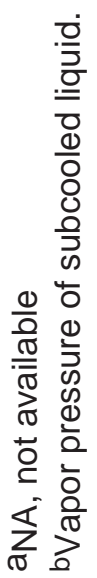




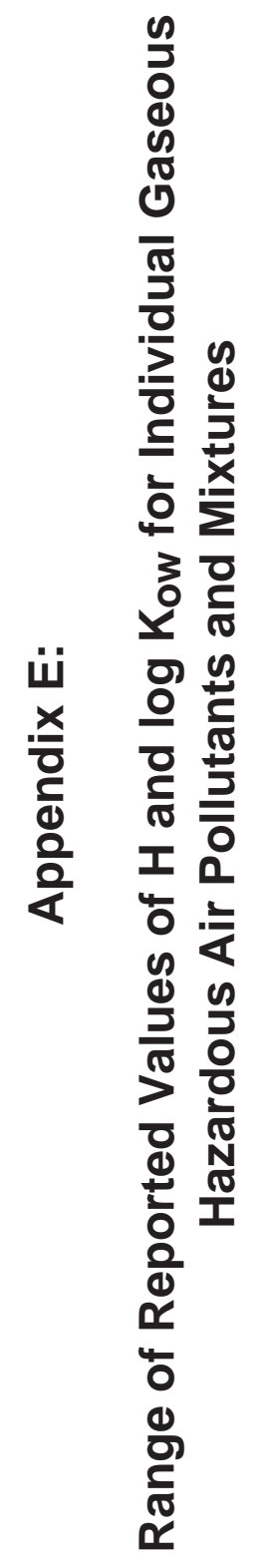




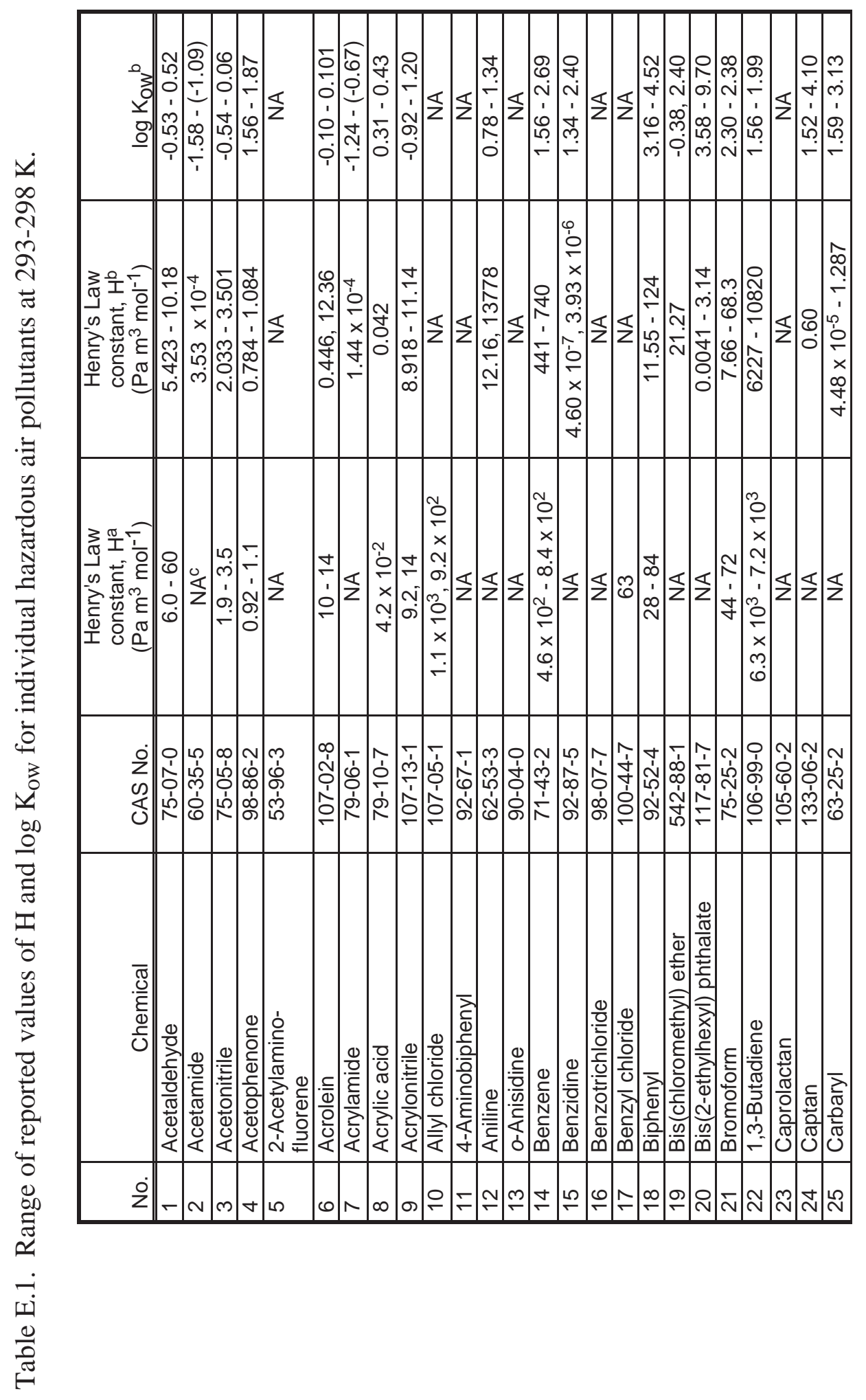




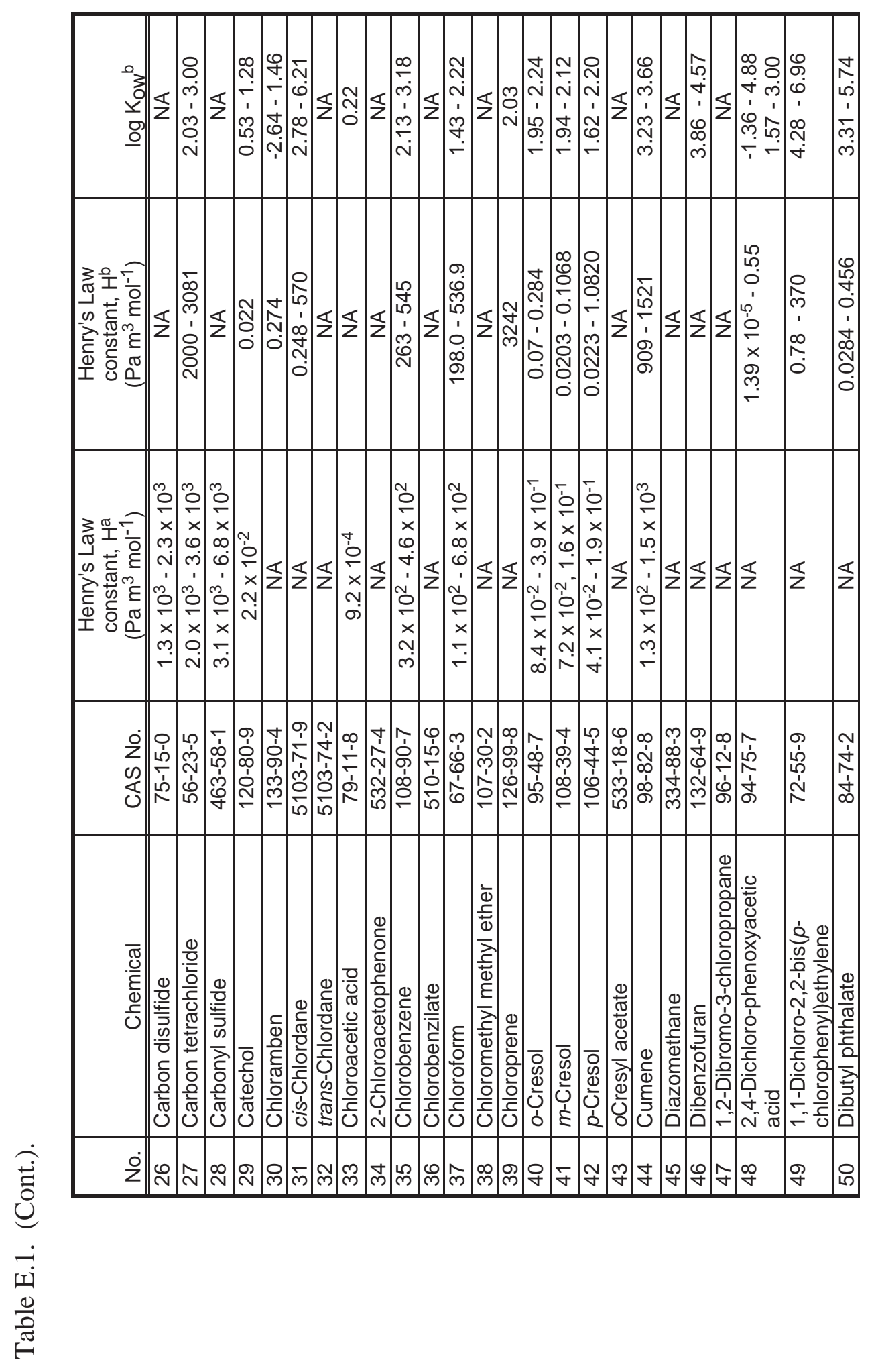




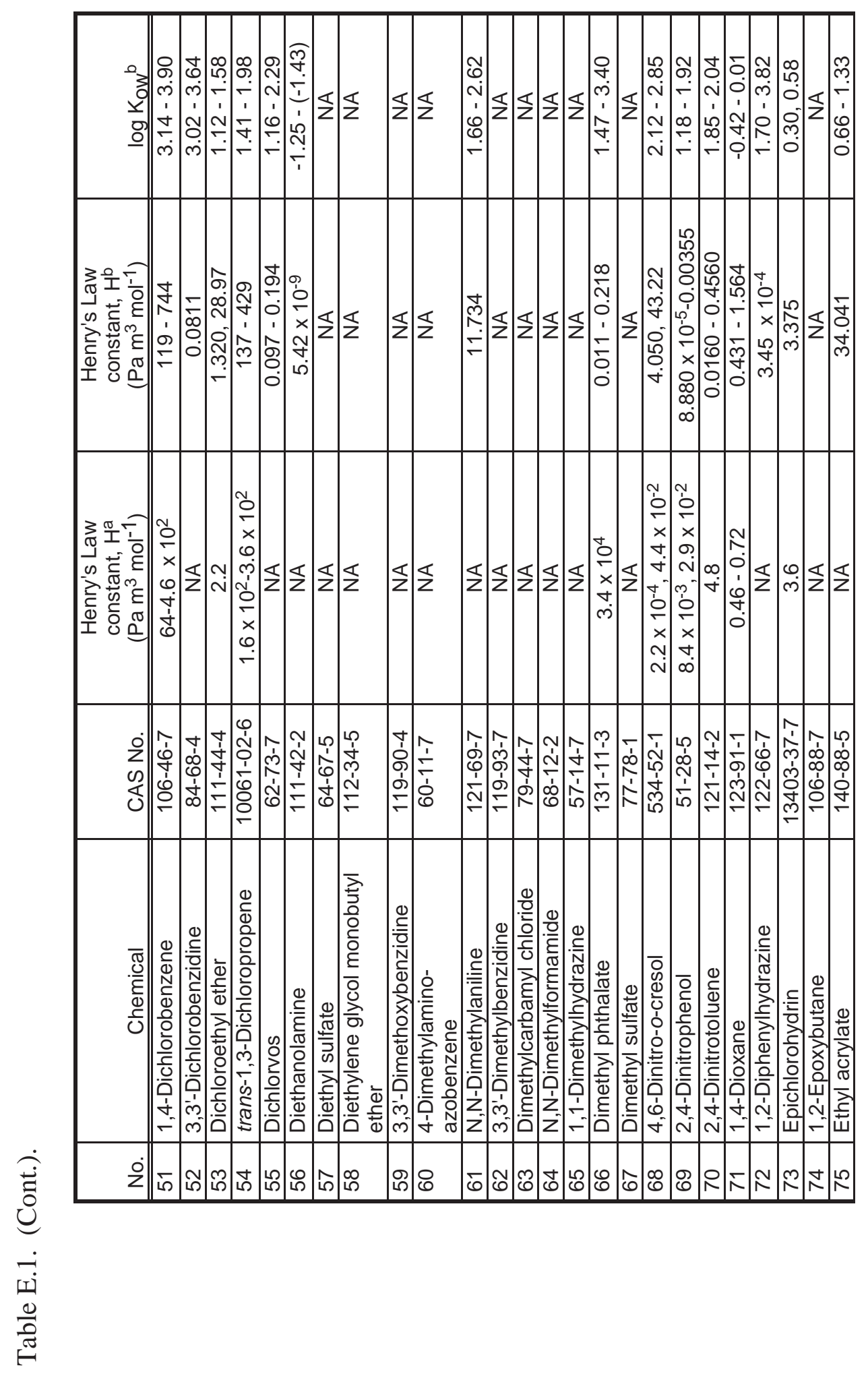




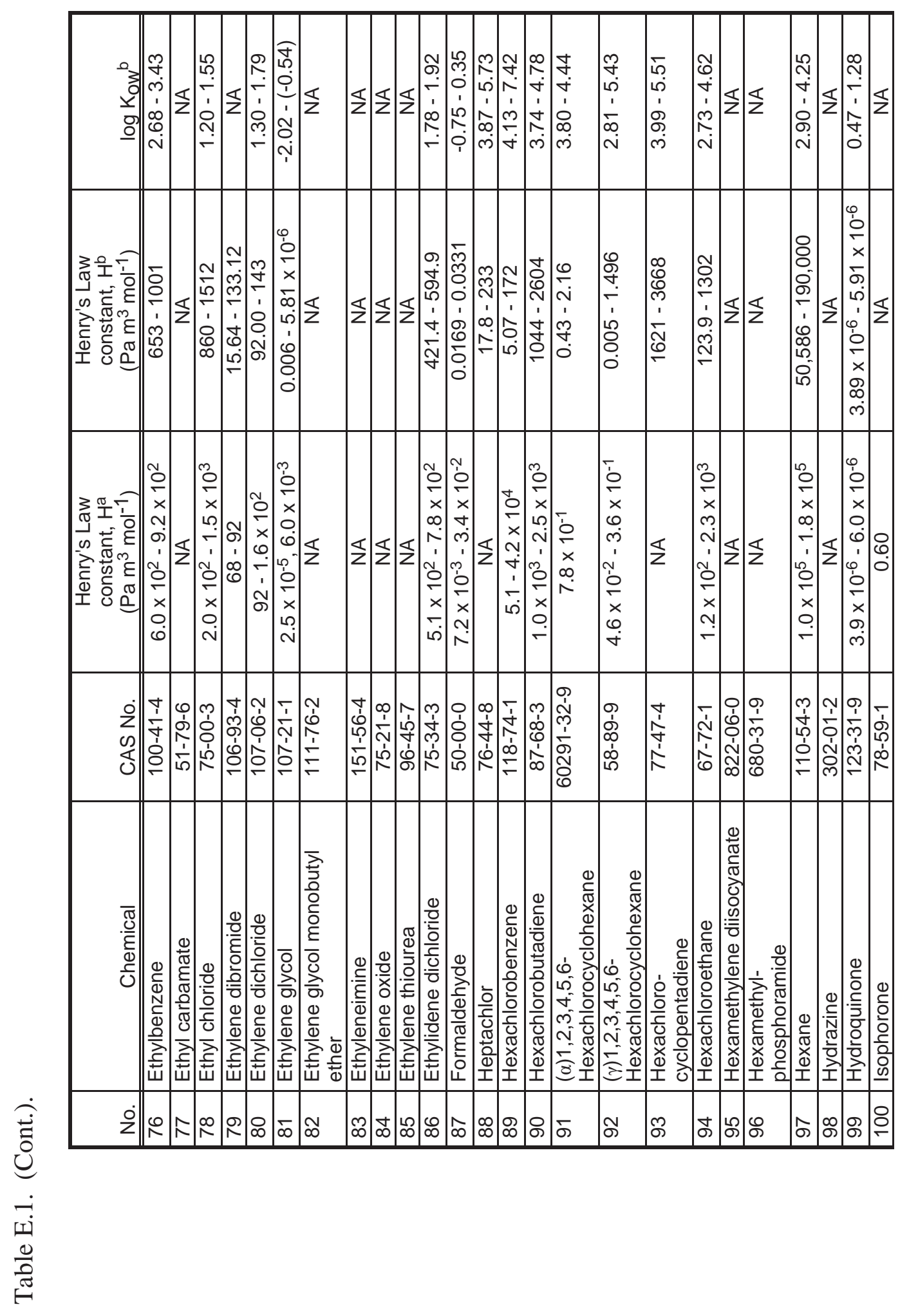




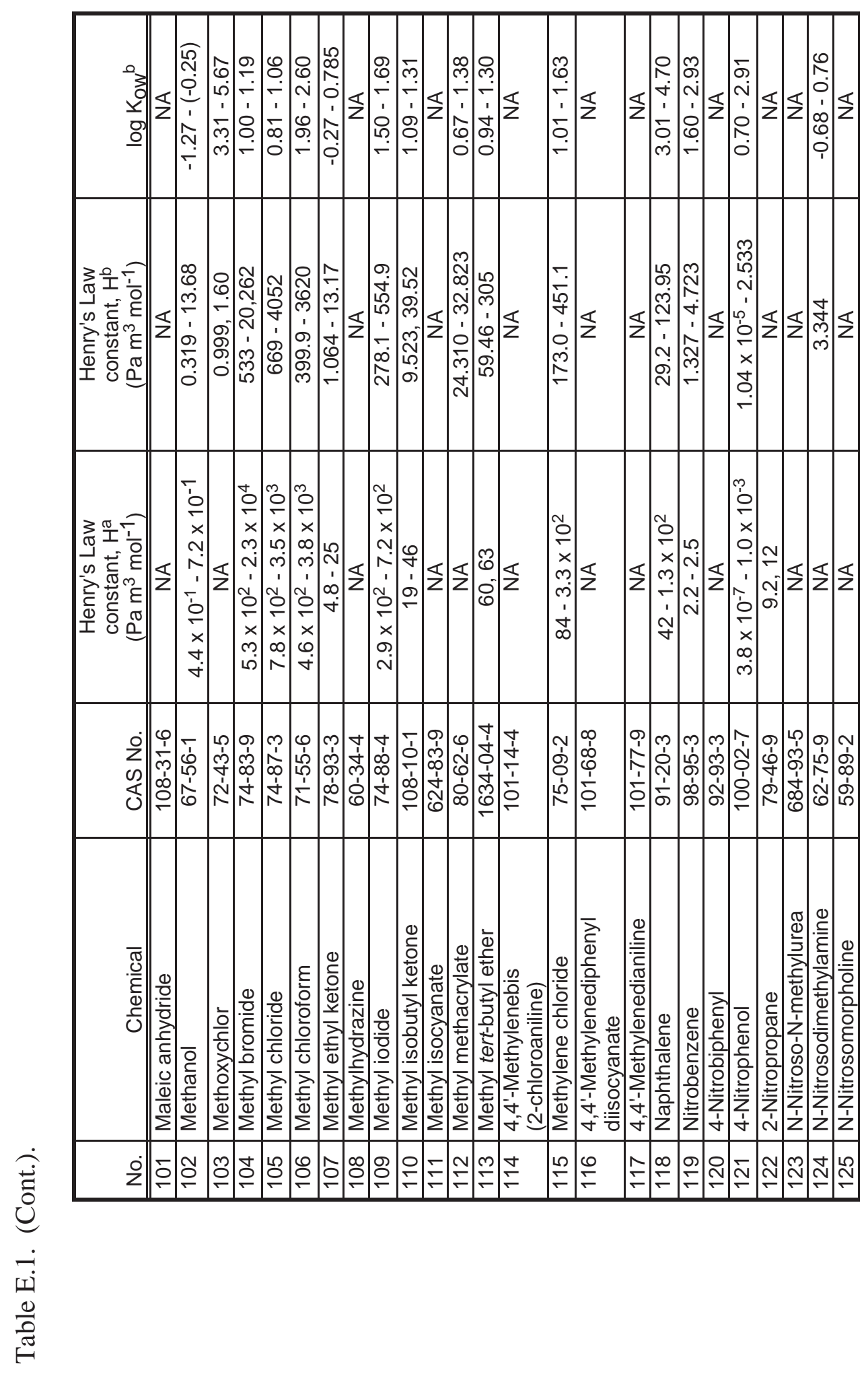




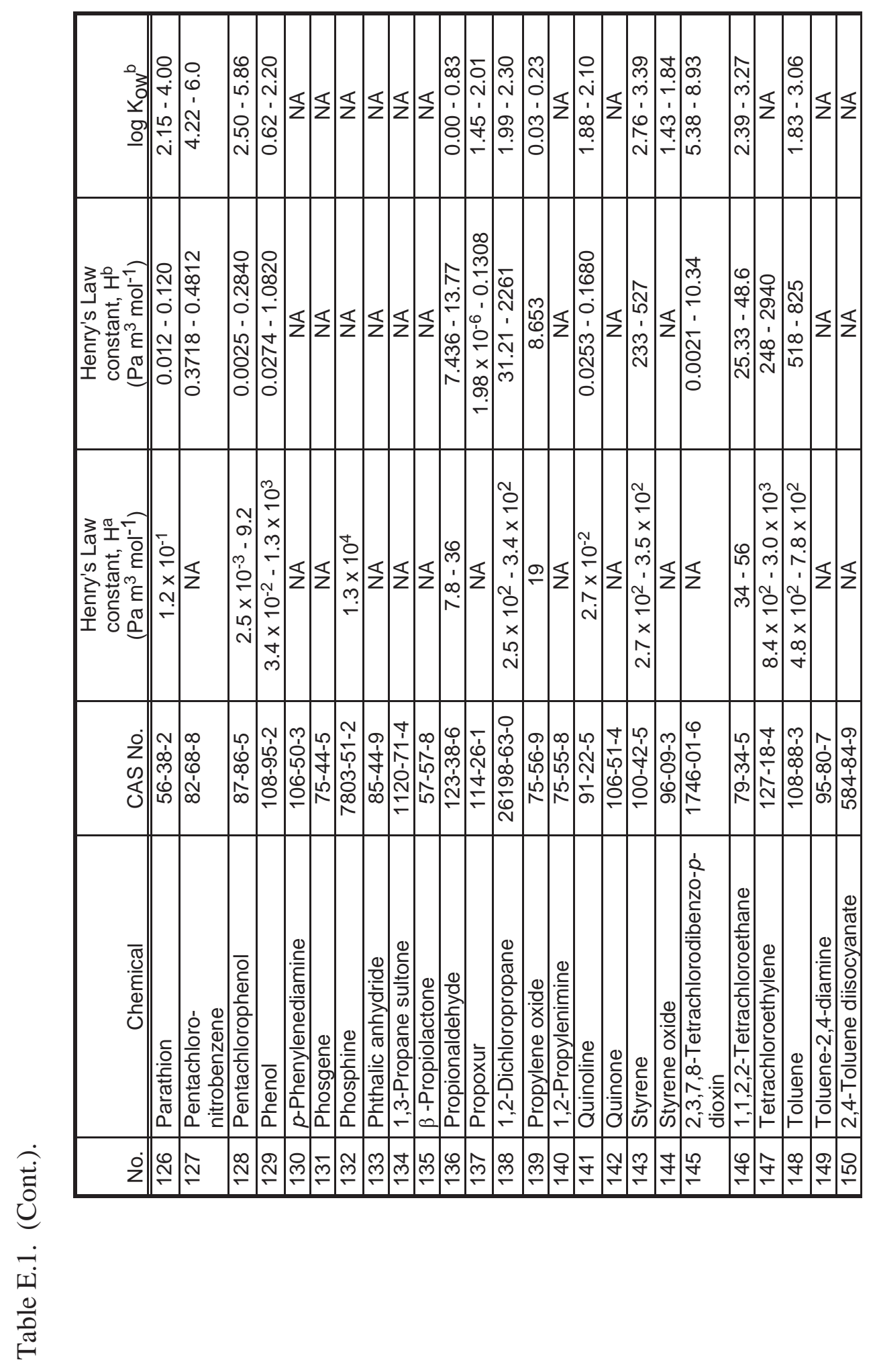




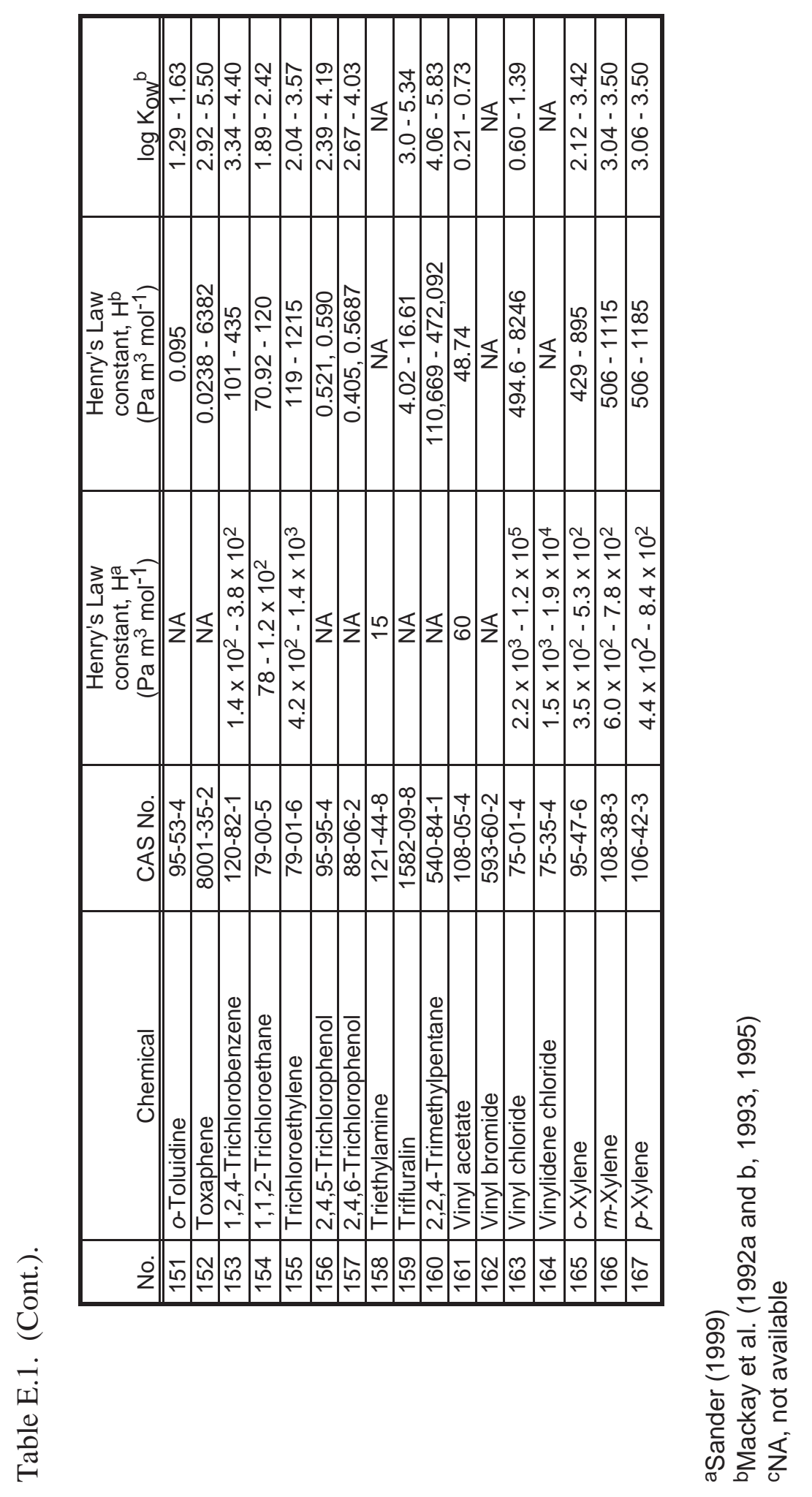




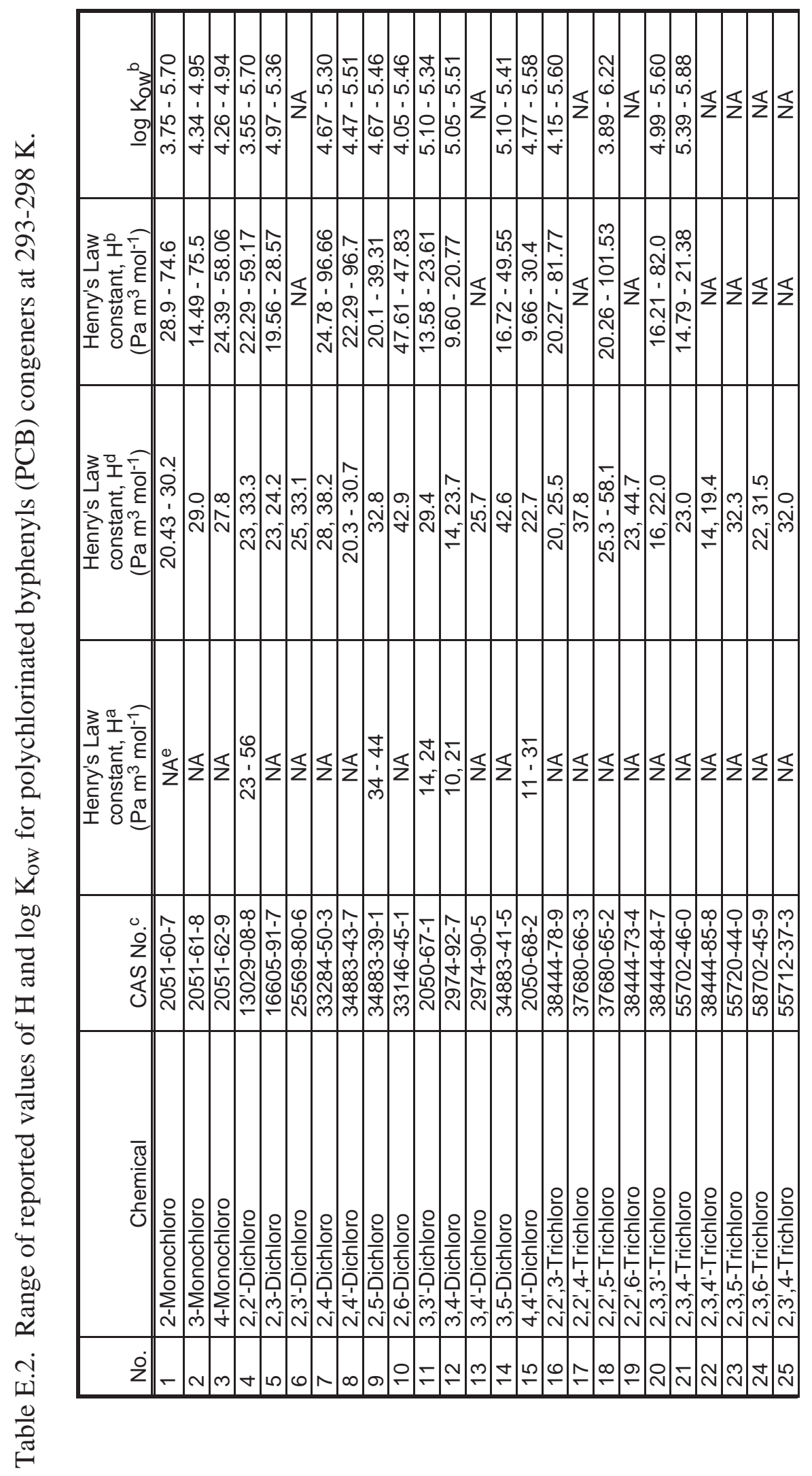




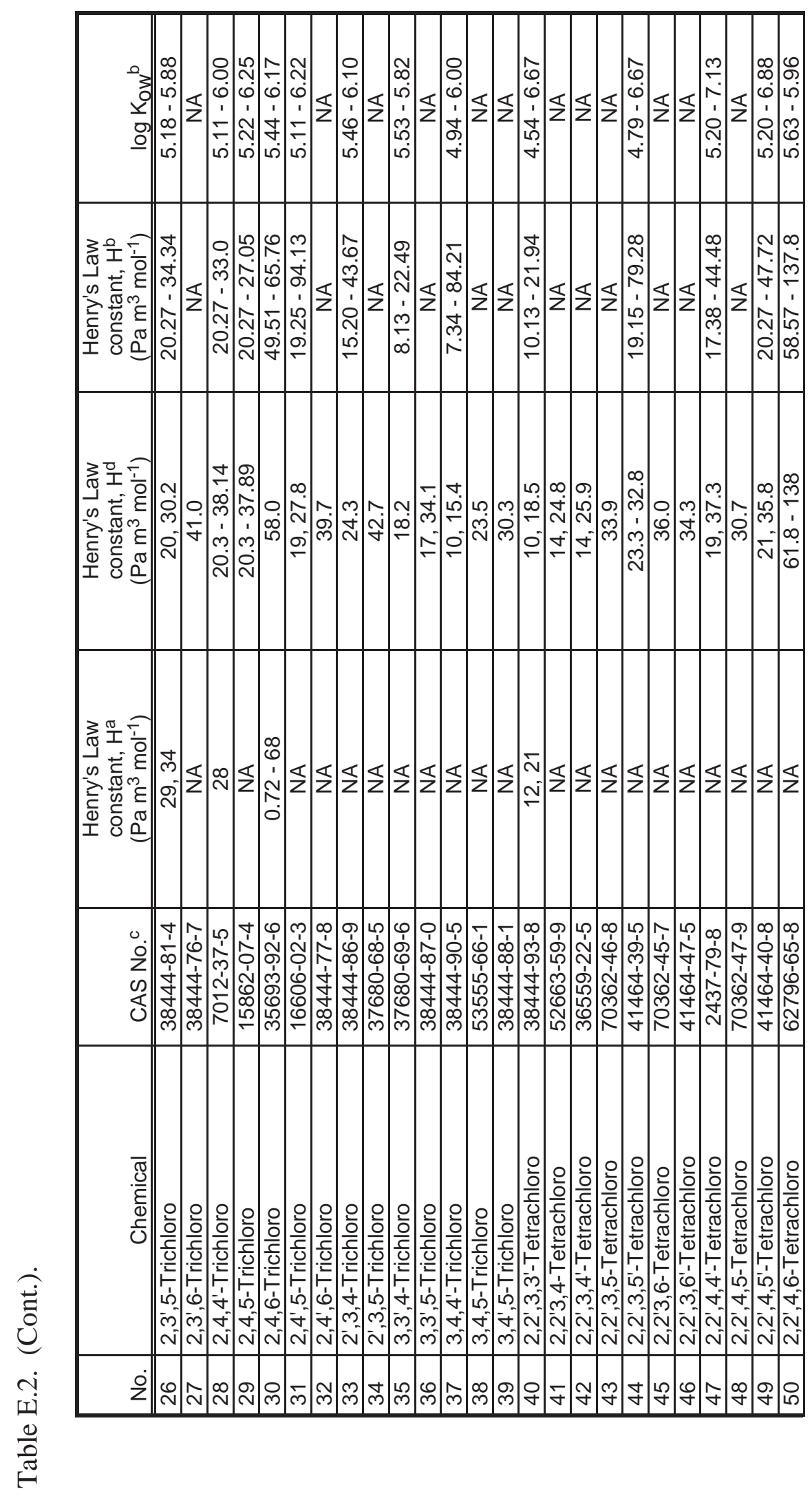

옴 


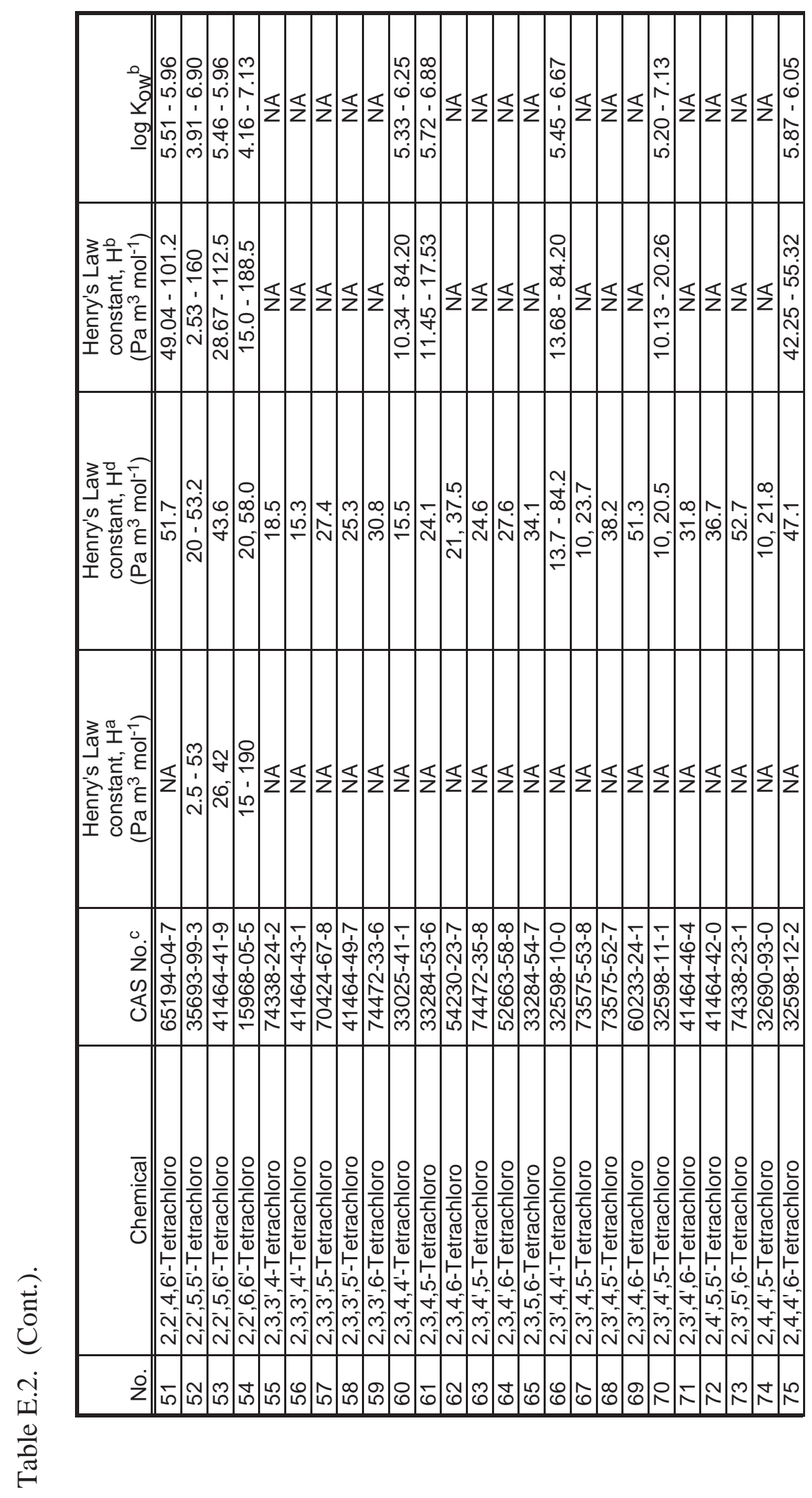




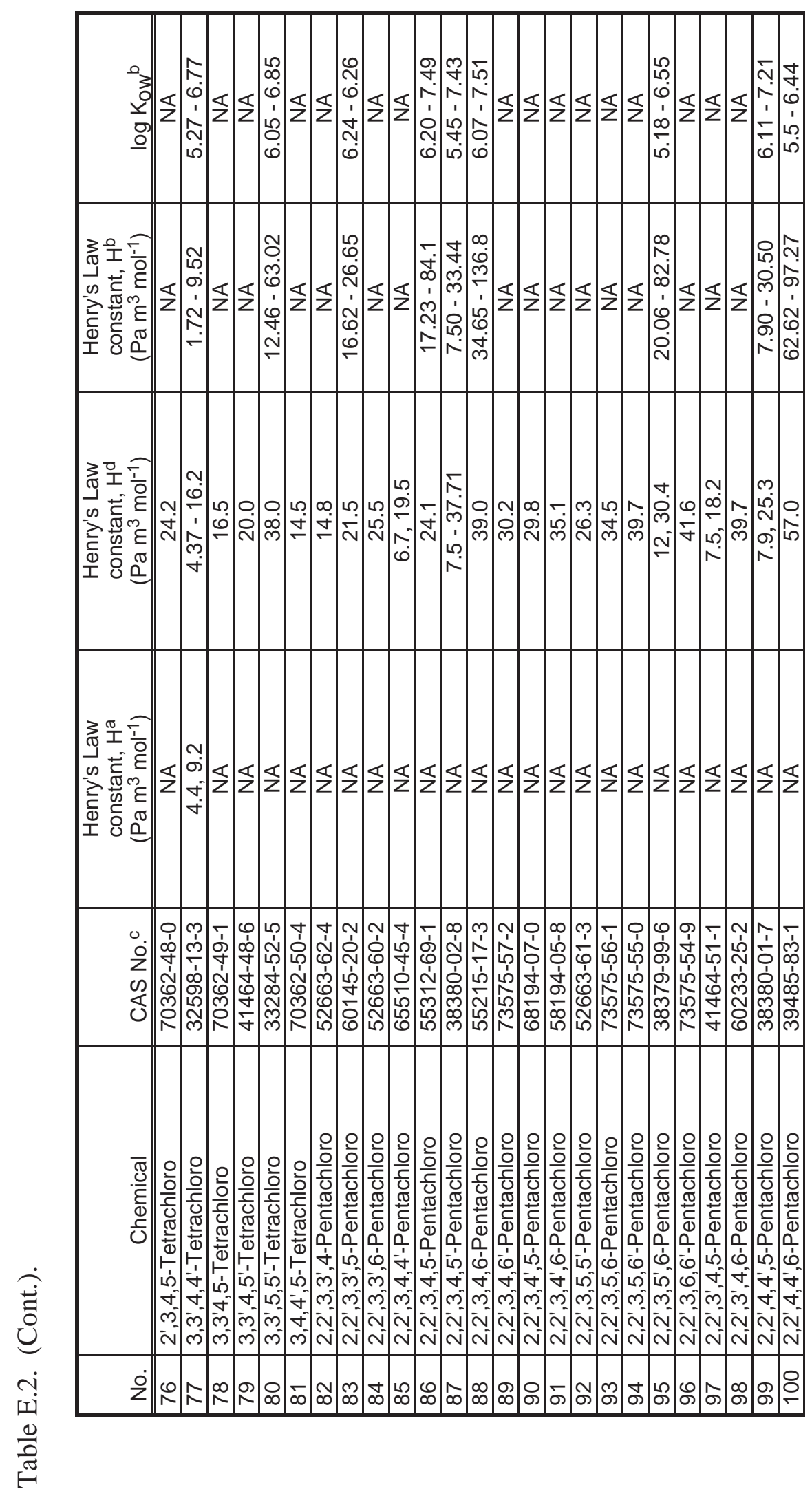

$\frac{7}{11}$ 


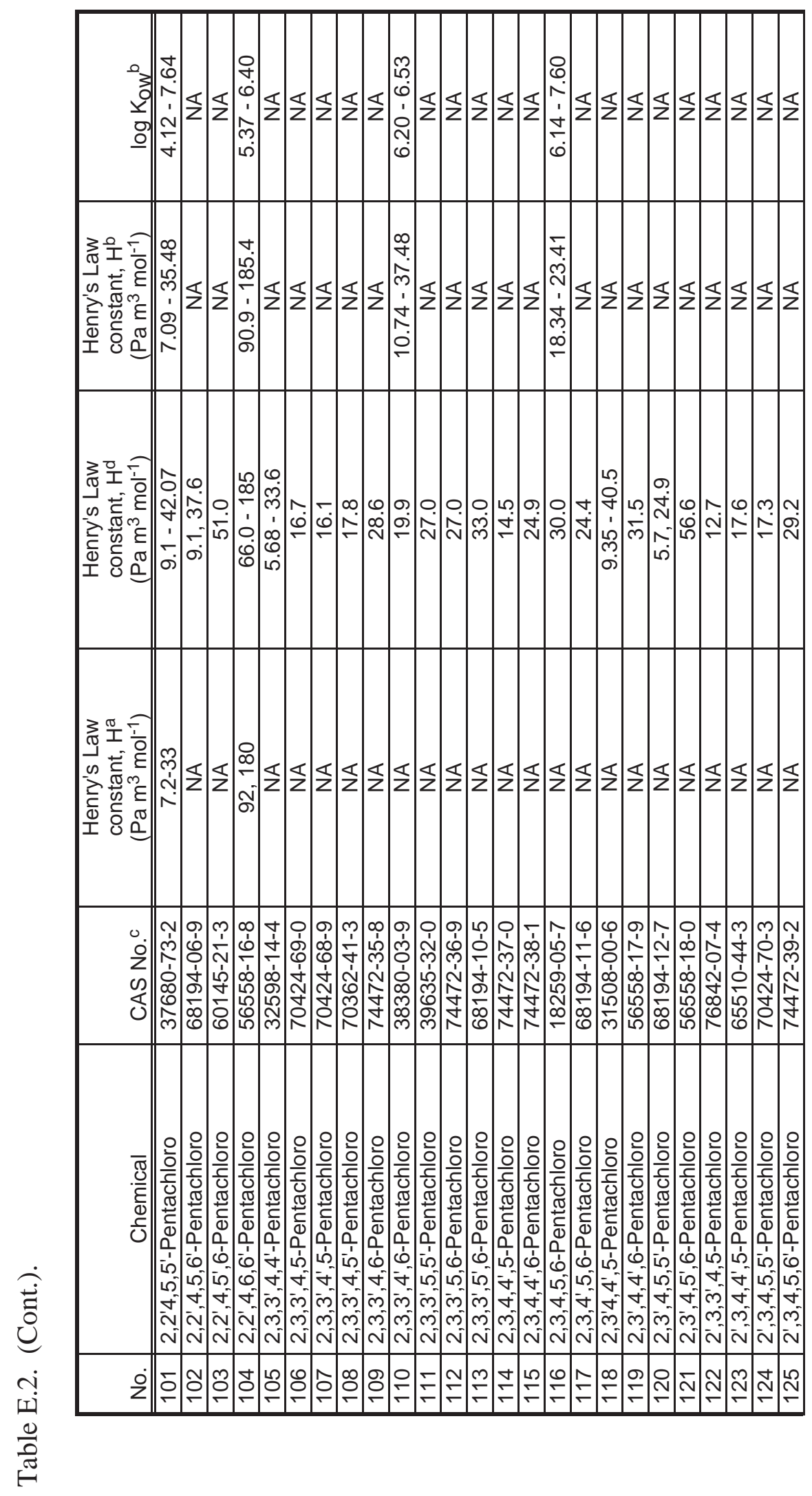

$\frac{m}{11}$ 


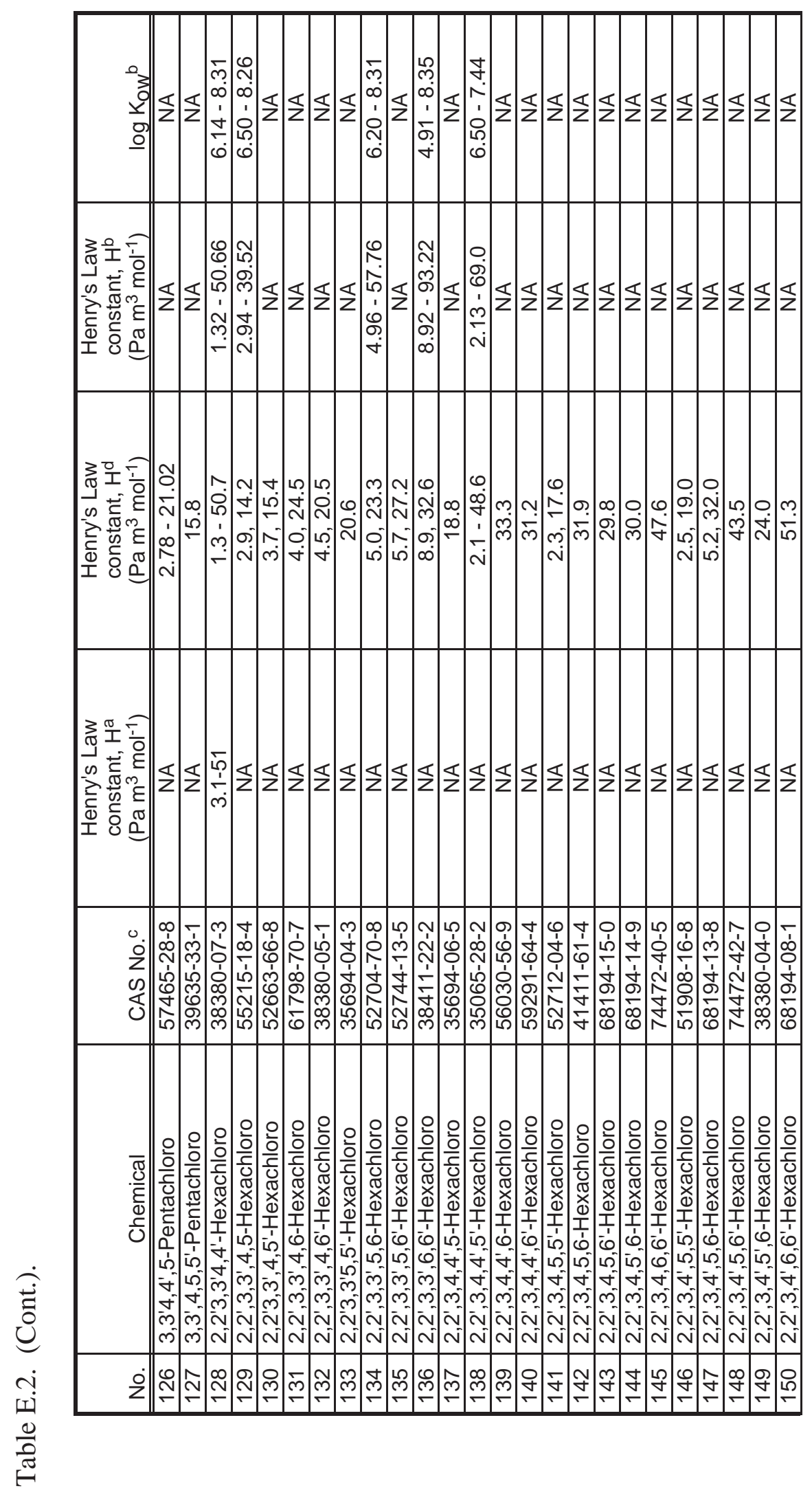




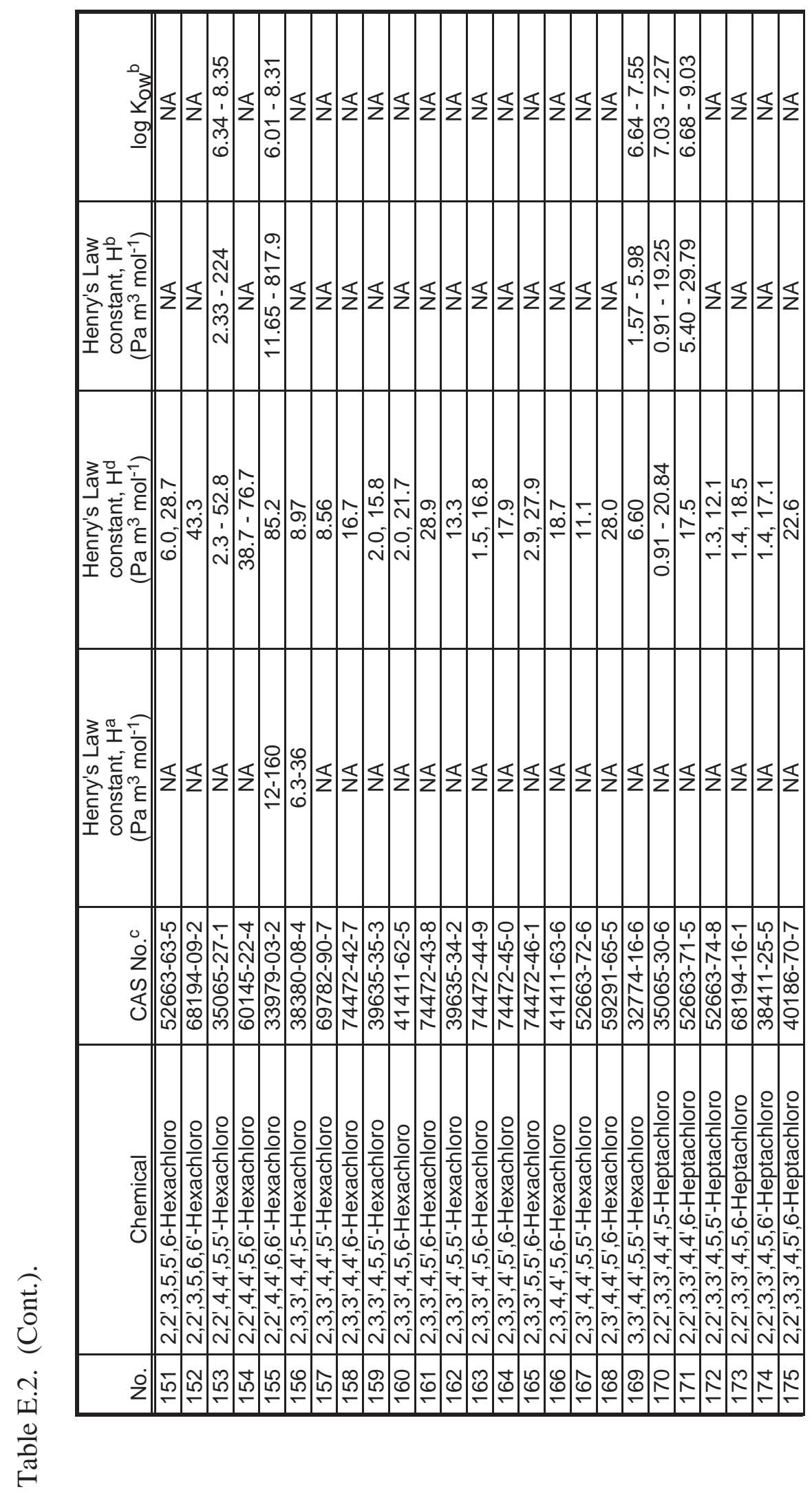




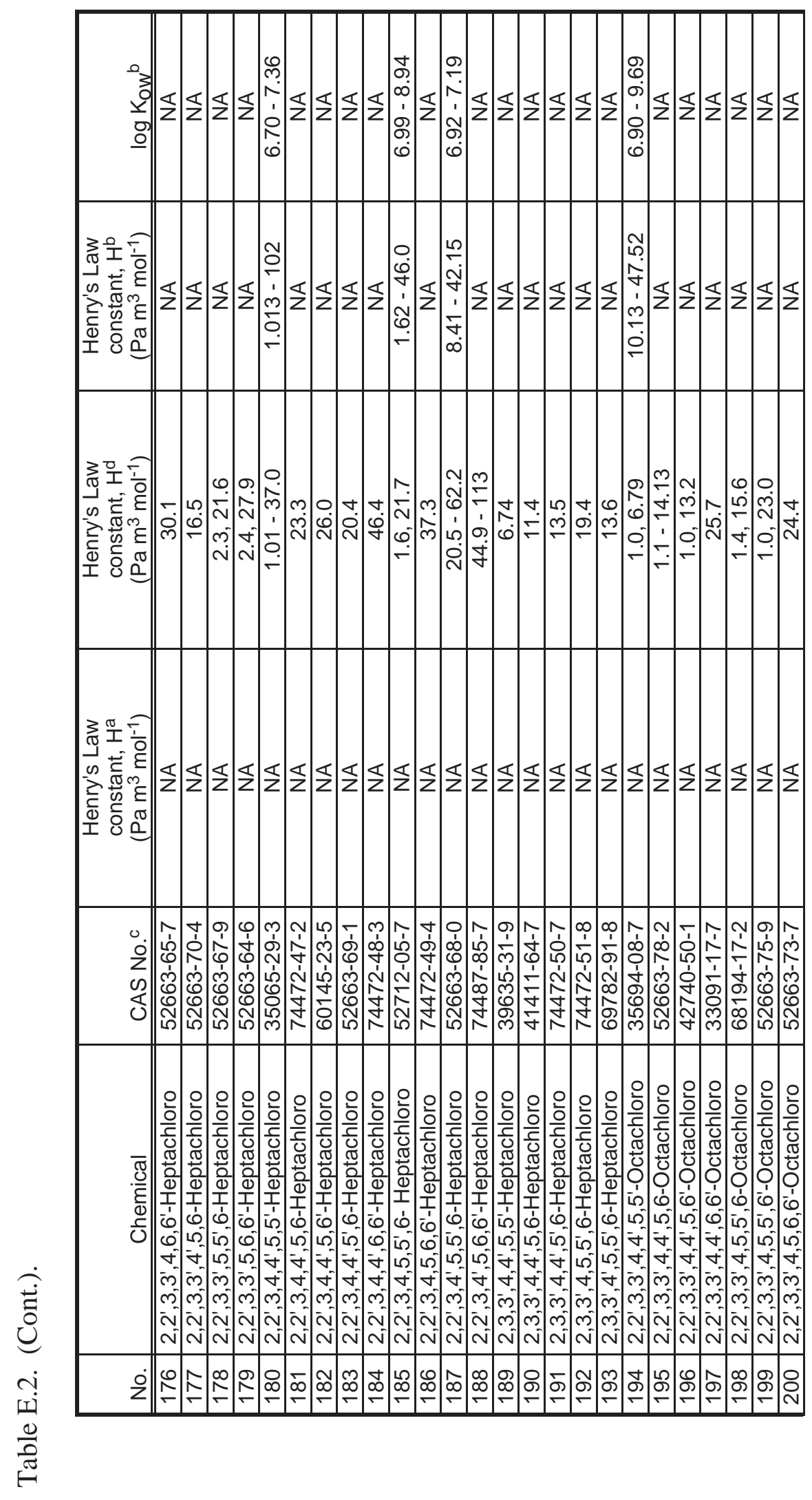

돈 


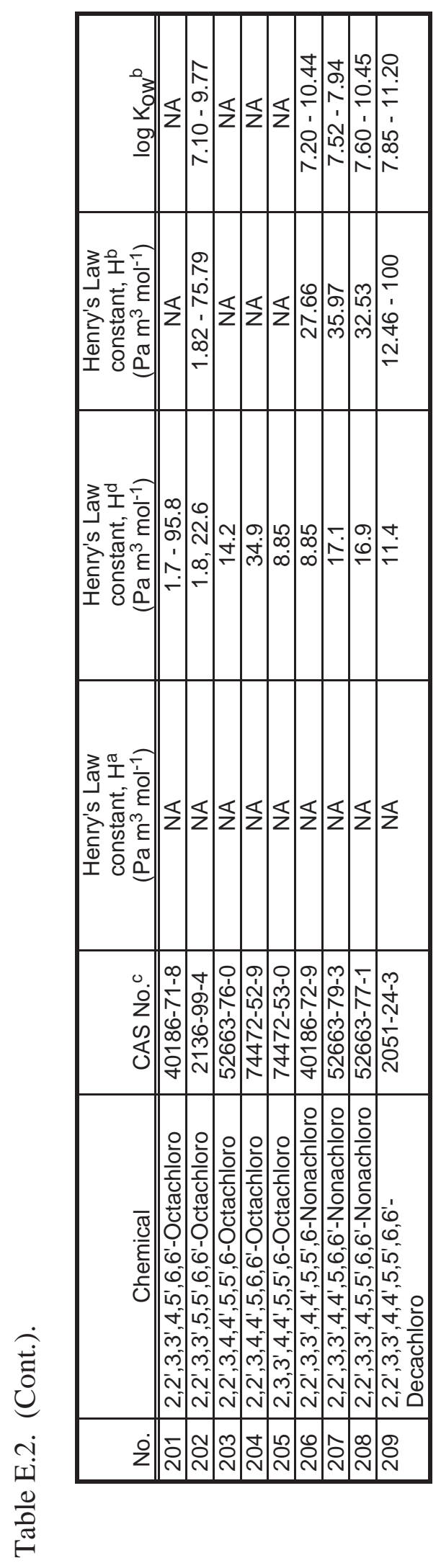

II

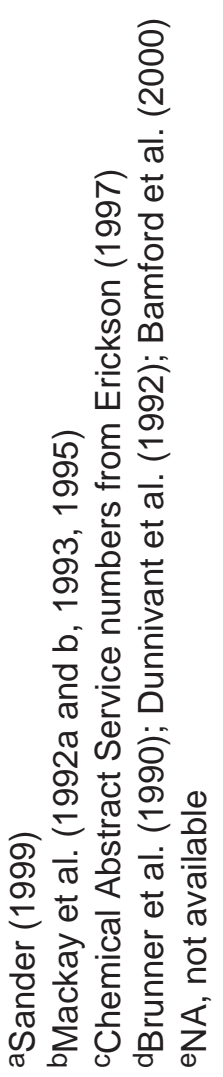




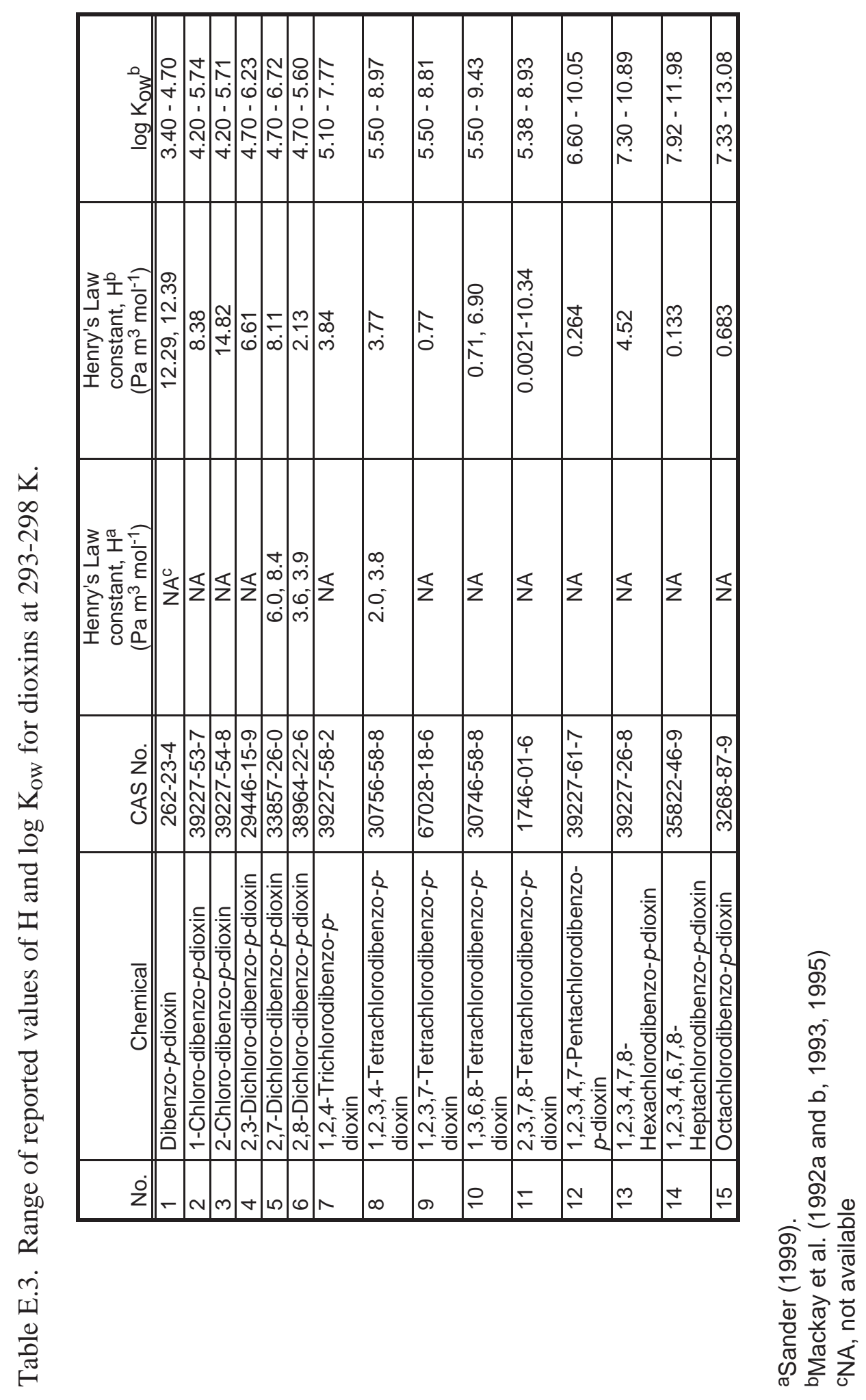

$\frac{\infty}{11}$ 


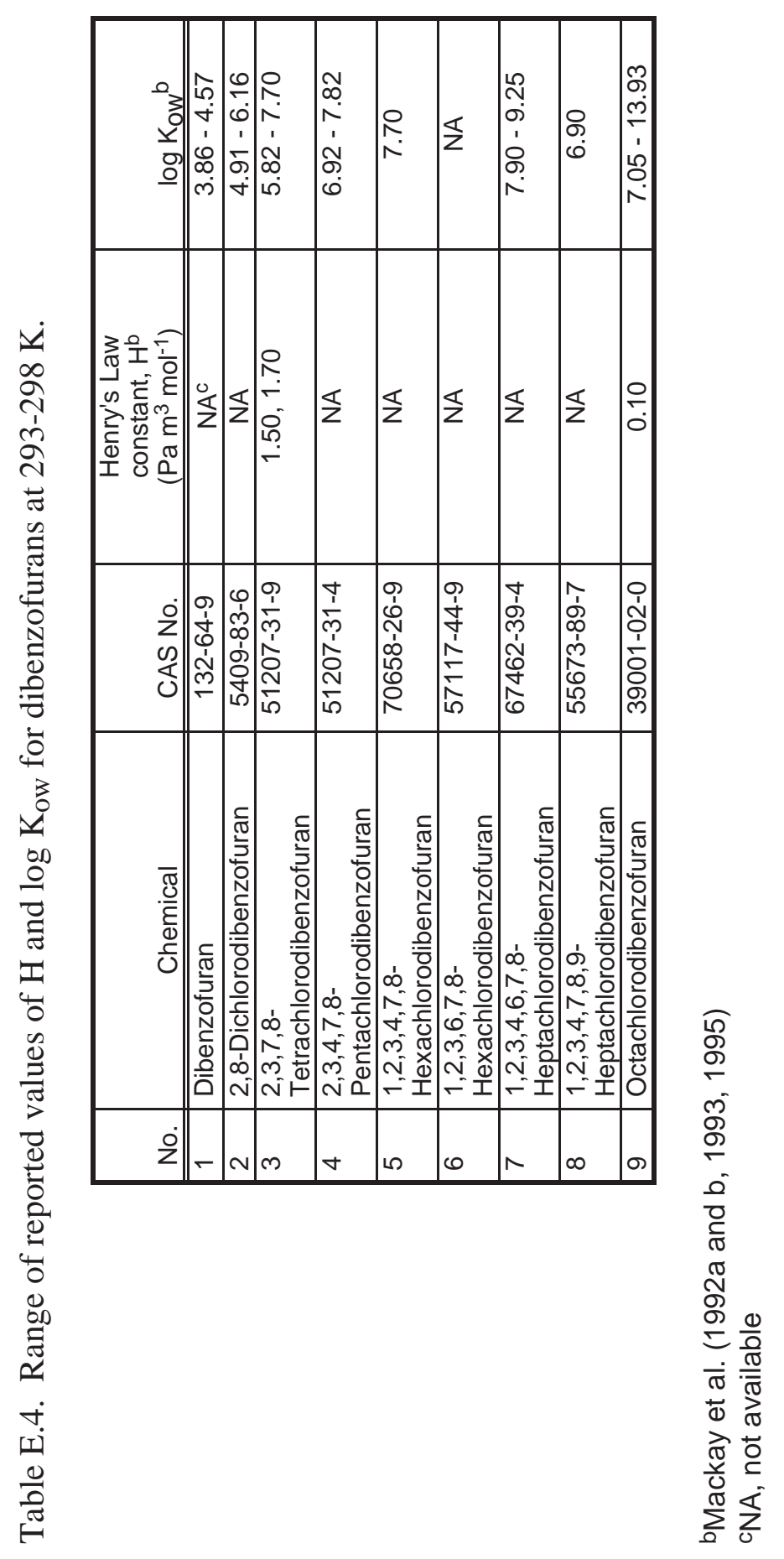

딤 


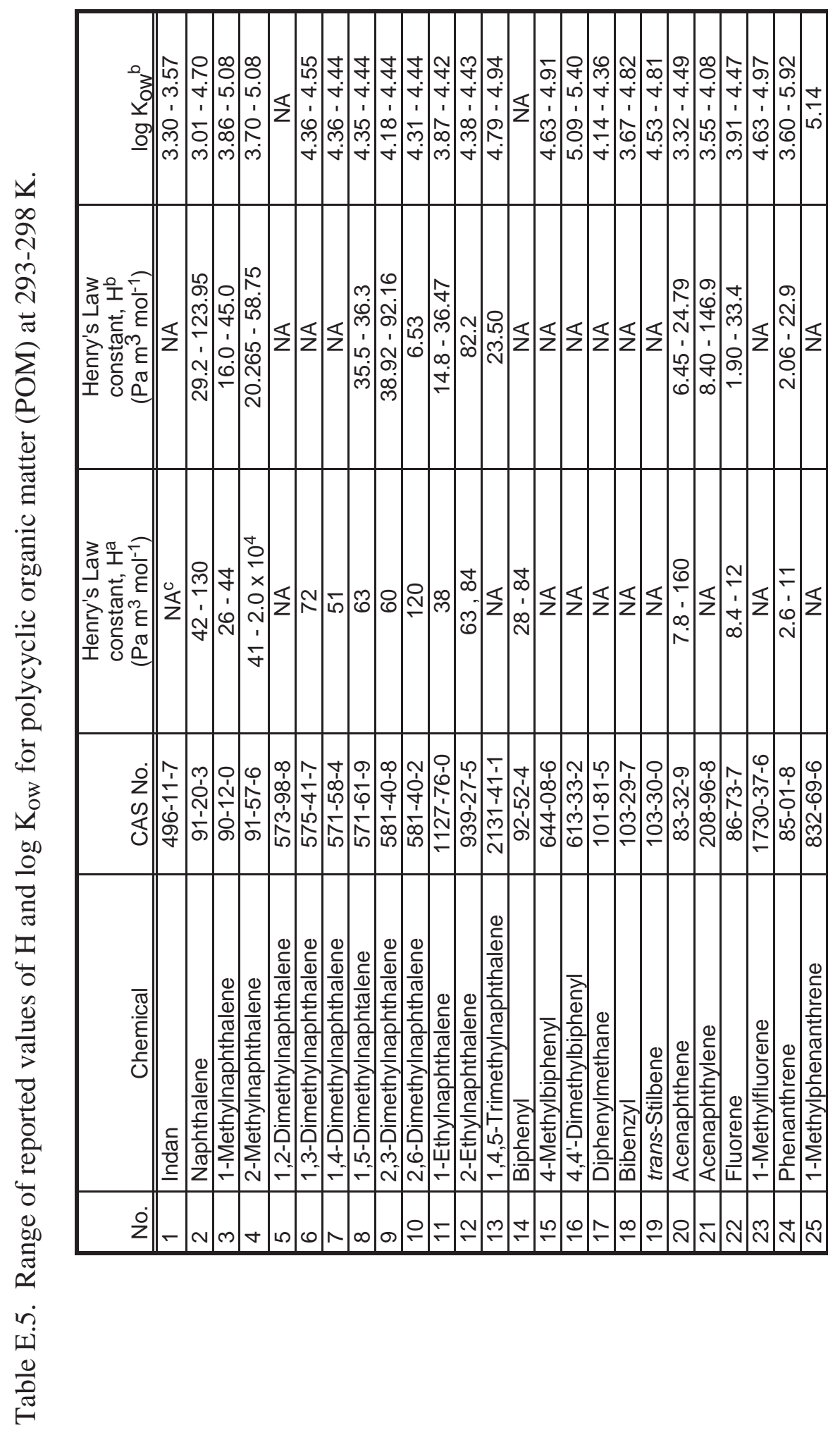




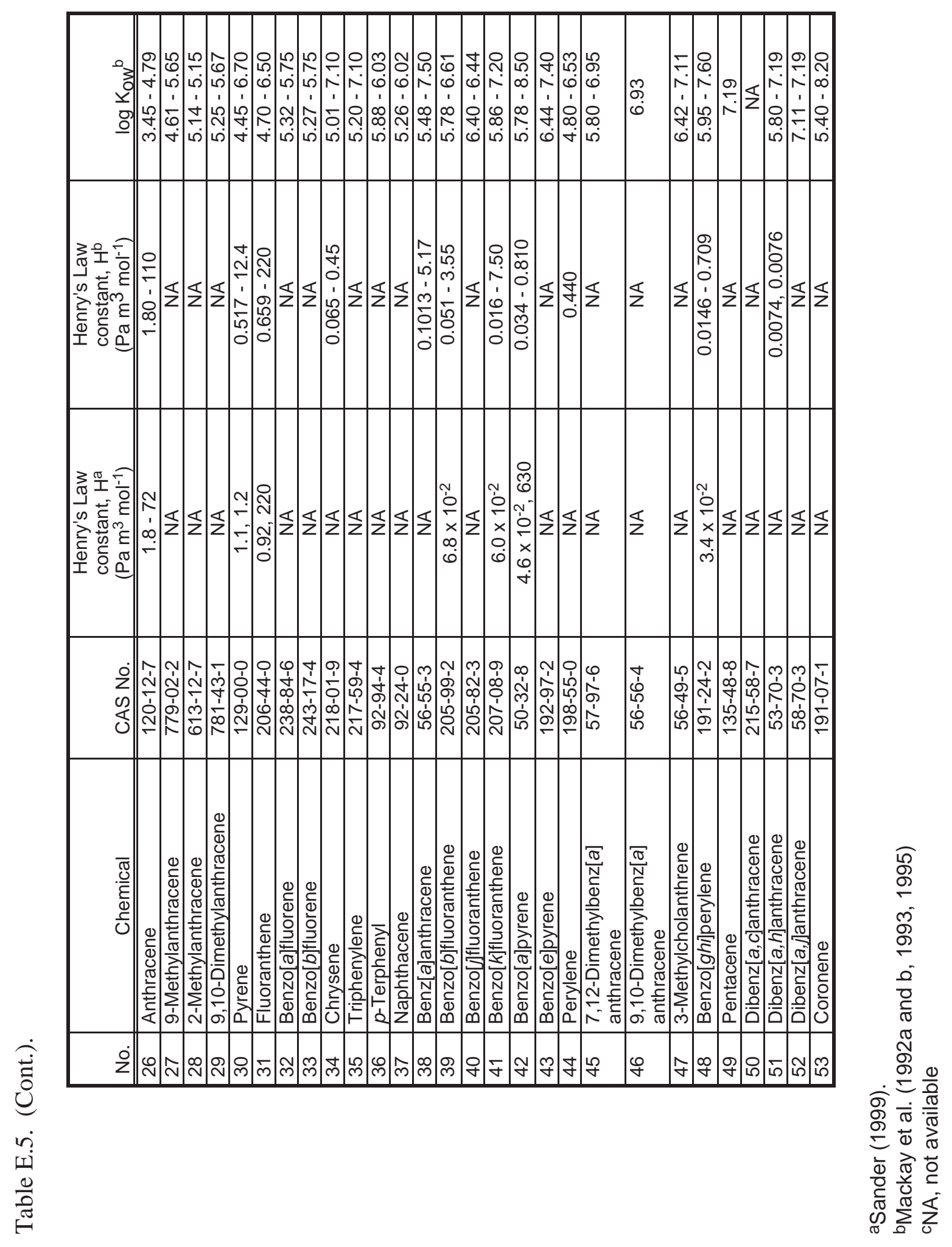




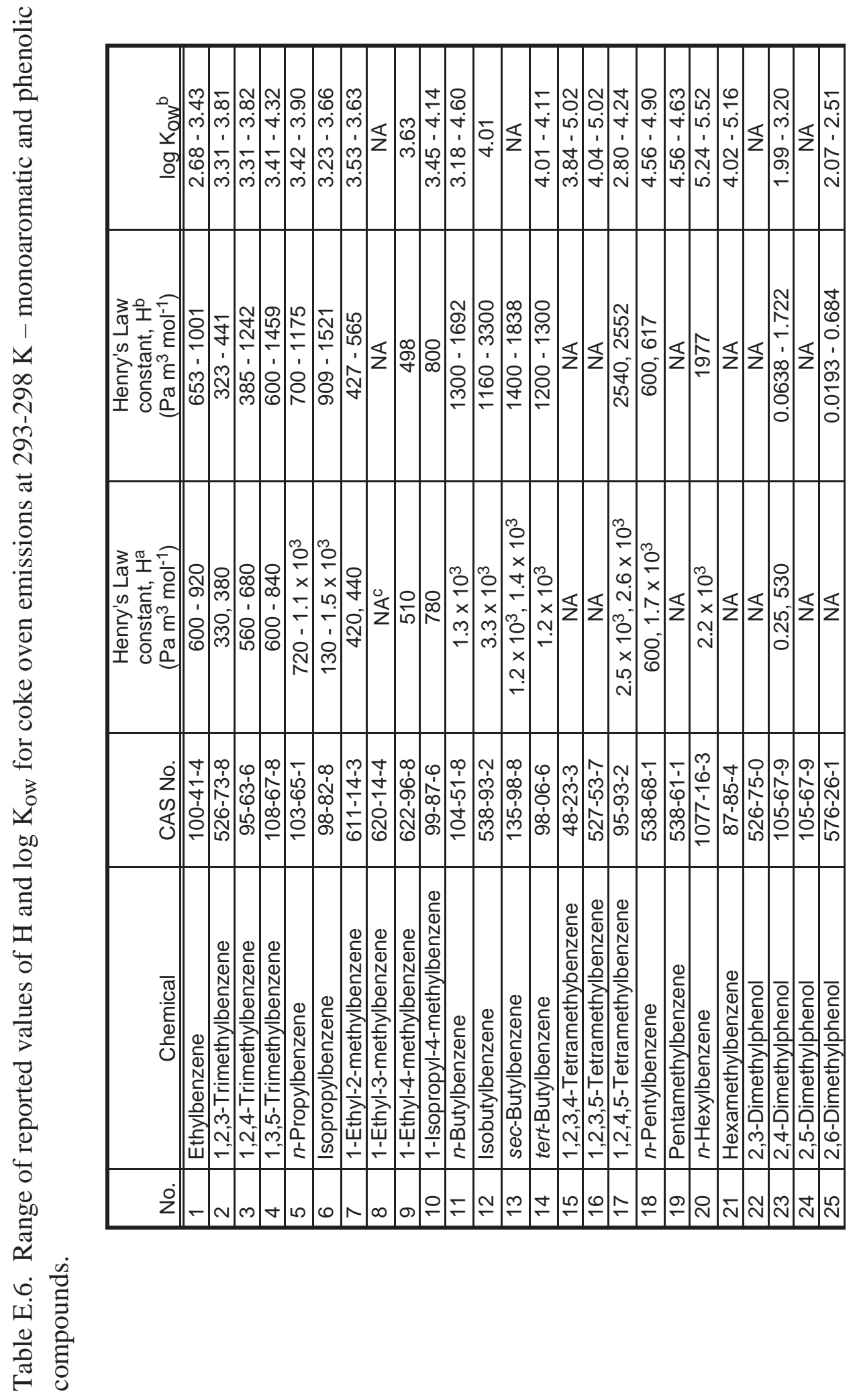




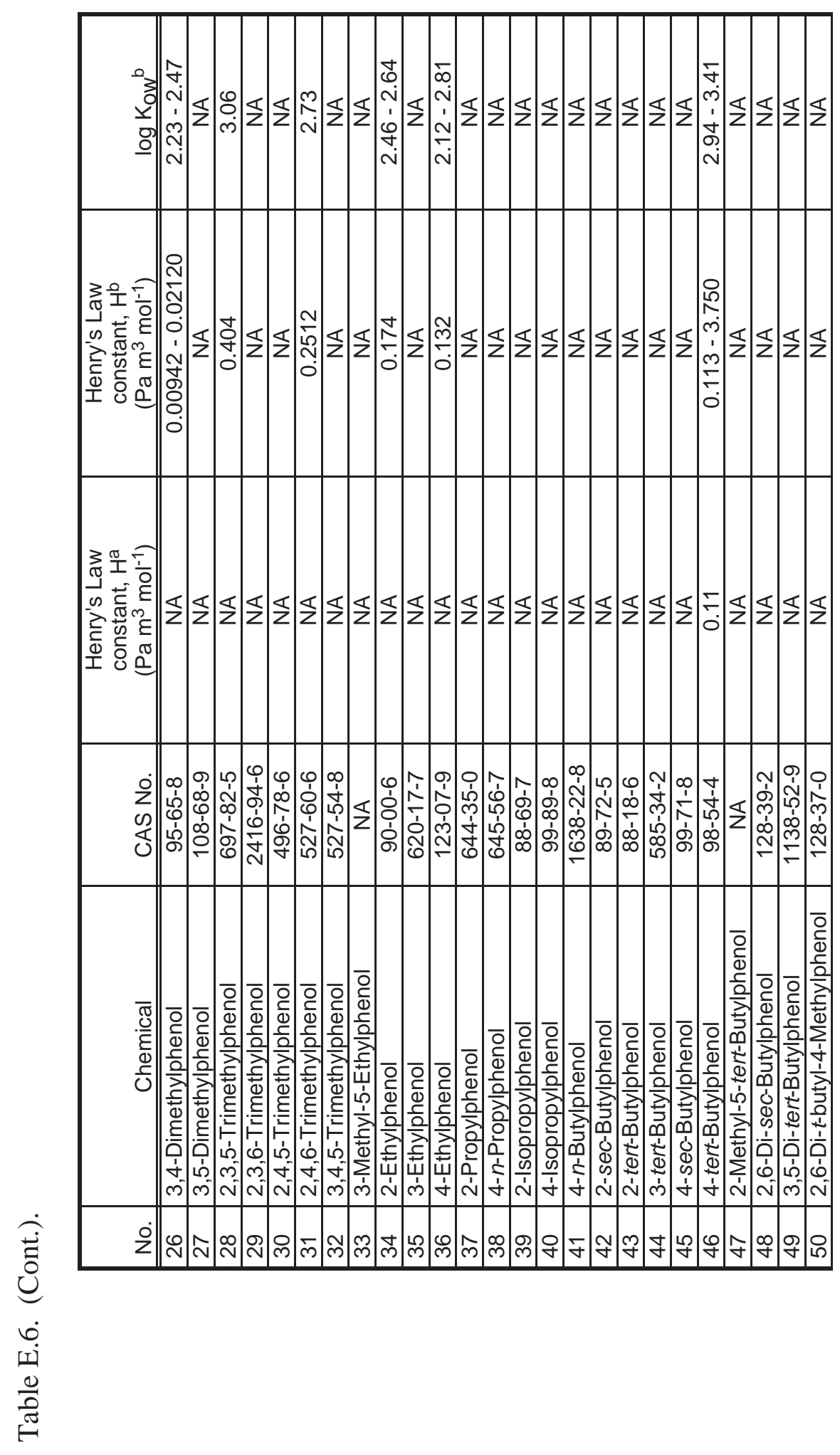

$\underset{\sim}{1}$ 


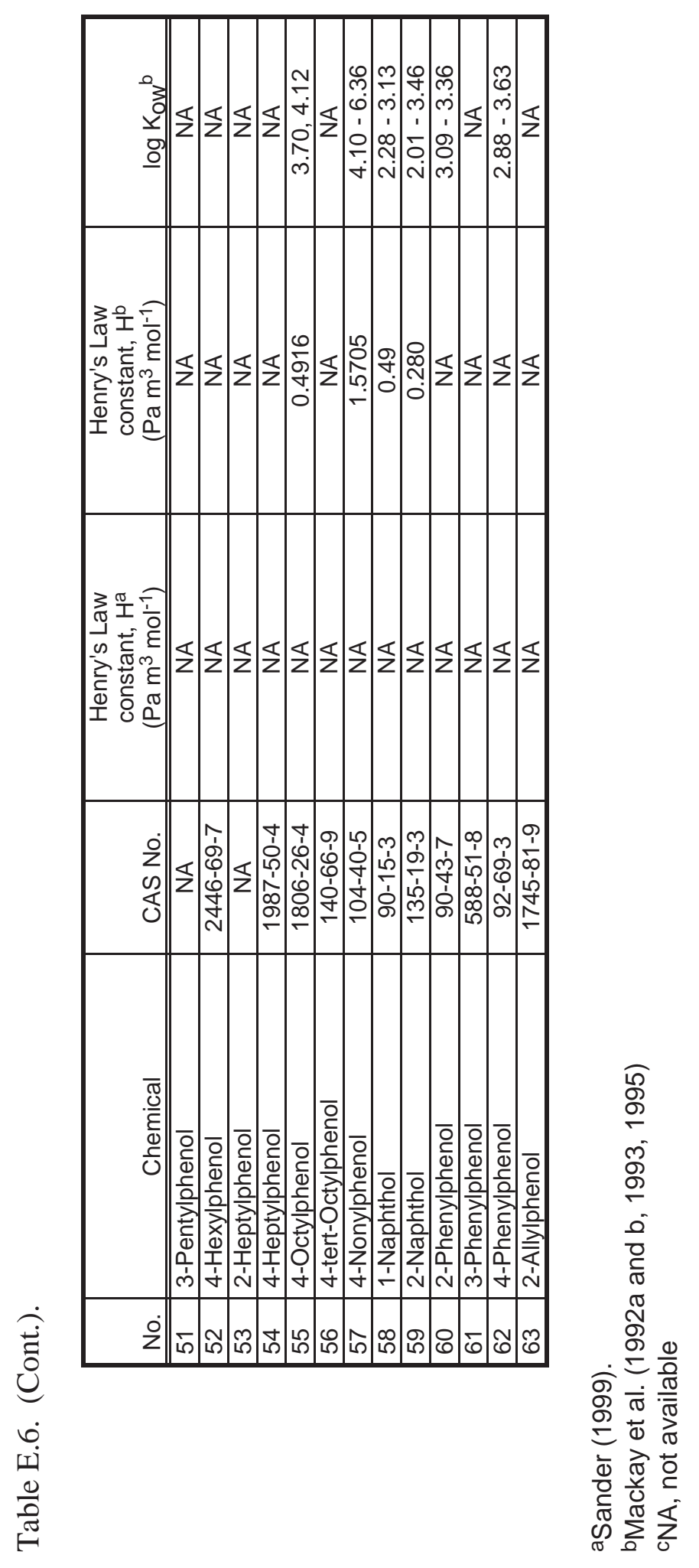

$\underset{\text { I }}{\stackrel{+}{1}}$ 


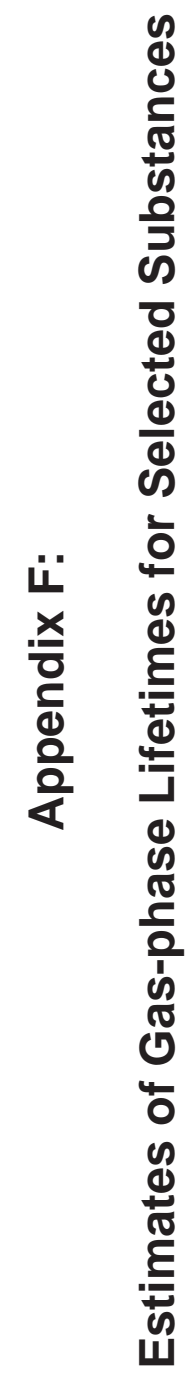




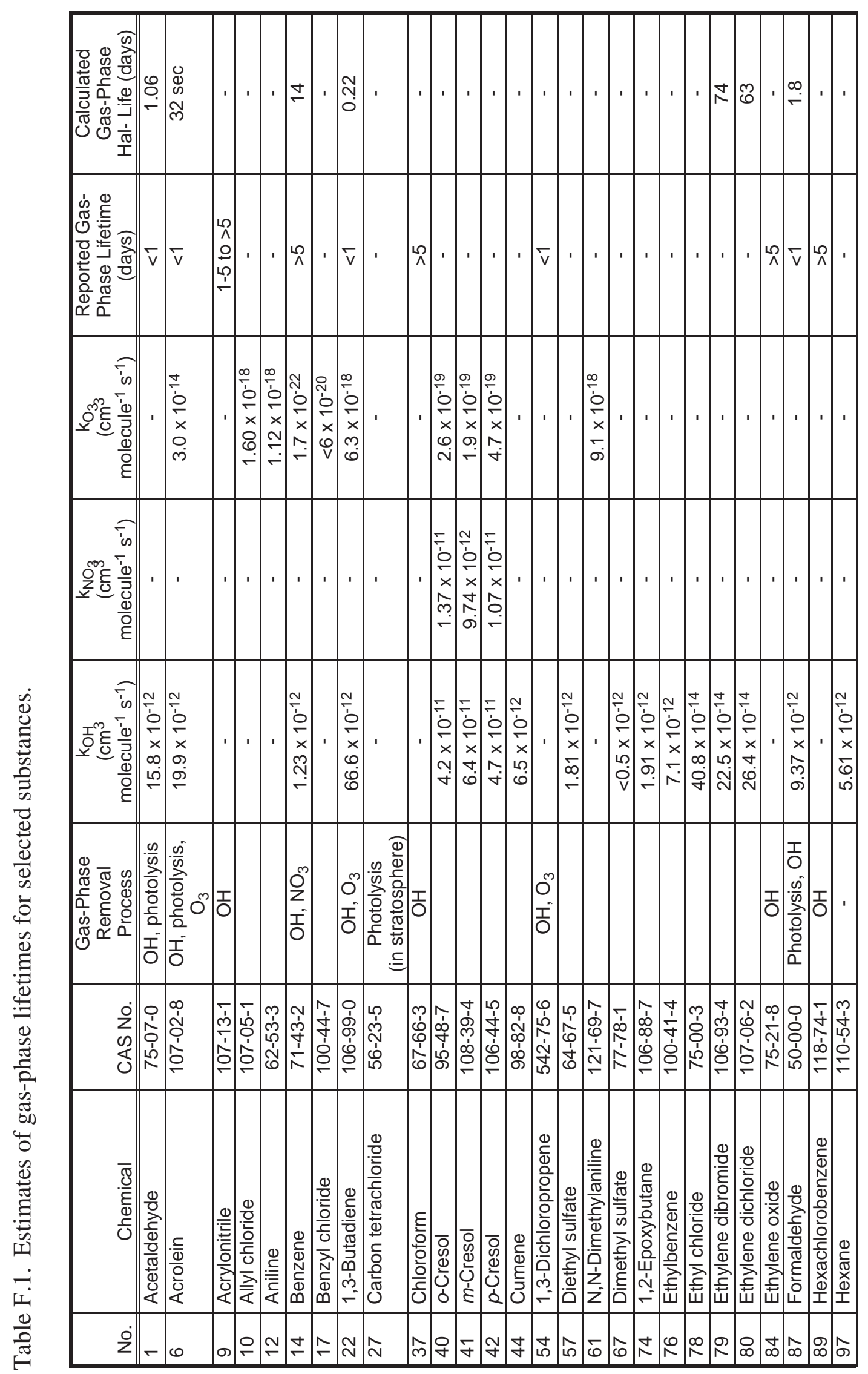




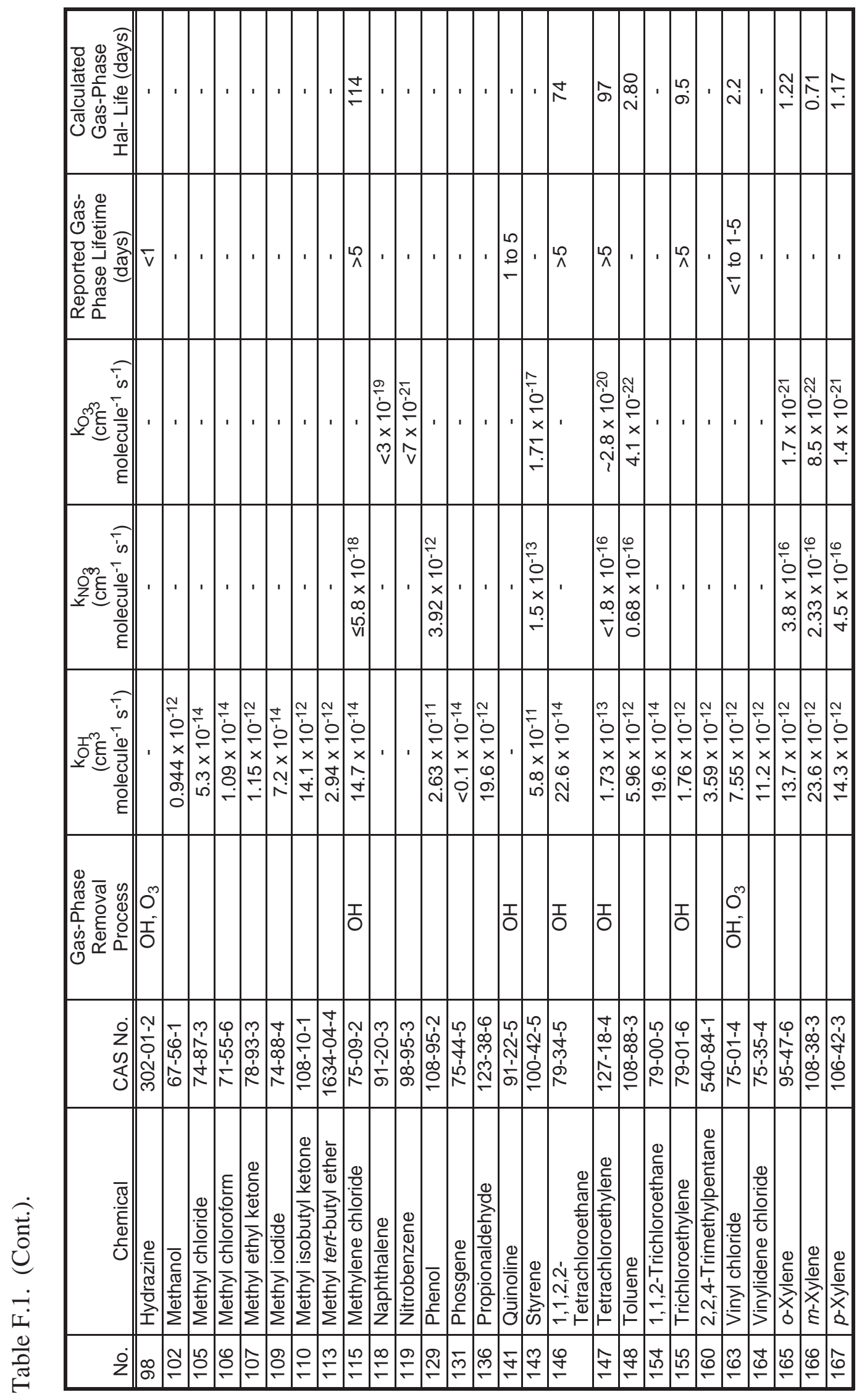

